\title{
Polybutadien und Butadien enthaltende Copolymere mit gezielt eingebauten vulkanisierbaren Gruppen durch RAFT-Polymerisation
}

\author{
Dissertation \\ zur Erlangung des mathematisch-naturwissenschaftlichen Doktorgrades \\ „Doctor rerum naturalium" \\ der Georg-August-Universität Göttingen \\ im Promotionsprogramm Chemie \\ der Georg-August University School of Science (GAUSS)
}

\author{
vorgelegt von \\ Cathrin Sonja Conrad \\ aus Hannover
}

Göttingen, 2013 


\section{Betreuungsausschuss}

Prof. Dr. Philipp Vana, MBA Institut für Physikalische Chemie, Georg-August-Universität Göttingen

Prof. Dr. Michael Buback Institut für Physikalische Chemie, Georg-August-Universität Göttingen

\section{Mitglieder der Prüfungskommission}

\section{Referent}

Prof. Dr. Philipp Vana, MBA Institut für Physikalische Chemie, Georg-August-Universität Göttingen

\section{Korreferent}

Prof. Dr. Michael Buback Institut für Physikalische Chemie, Georg-August-Universität Göttingen

\section{Weitere Mitglieder der Prüfungskommission}

Prof. Dr. Götz Eckold

Prof. Dr. Peter Botschwina

Prof. Dr. Burkhard Geil
Institut für Physikalische Chemie, Georg-August-Universität Göttingen Institut für Physikalische Chemie, Georg-August-Universität Göttingen Institut für Physikalische Chemie, Georg-August-Universität Göttingen

Institut für Organische und Biomolekulare Chemie, Georg-August-Universität Göttingen

Tag der mündlichen Prüfung: 29.10.2013 

Die vorliegende Arbeit wurde in der Zeit von Oktober 2009 bis September 2013 am Institut für Physikalische Chemie der Georg-August-Universität Göttingen unter der Leitung von Prof. Dr. Philipp Vana in einer Kooperation mit der Continental Reifen $\mathrm{GmbH}$, Hannover, angefertigt. 


\section{Zusammenfassung}

Im Rahmen dieser Arbeit wurden Möglichkeiten für die Funktionalisierung von Polybutadien und Butadien enthaltenden Copolymeren mit gezielt eingebauten potenziell vulkanisierbaren Gruppen für technische Anwendungen erarbeitet. Dabei standen zwei verschiedene Ansätze im Vordergrund: Die $\alpha, \omega$-Funktionalisierung der Polymerketten mittels modifizierter RAFT-Agenzien, um die Integration der Kettenenden in das Polymernetzwerk zu gewährleisten, sowie eine Funktionalisierung entlang der Polymerkette mit Hilfe von speziellen Monomeren, um gezielt Vernetzungspunkte zu schaffen. Da diese Polymere in technische Anwendungen Verwendung finden sollen, standen einfache Synthesen, die sich gut auf den Technikums- und Industriemaßstab übertragen lassen, im Vordergrund.

Für die Funktionalisierung der beiden Kettenenden wurde die RAFT-Polymerisation (,Reversible Addition-Fragmentation Chain Transfer“) verwendet, da idealerweise jede auf diese Art hergestellte Polymerkette eine RAFT-Einheit trägt und so eine vollständige Funktionalisierung gewährleistet ist. Da es sich bei der RAFT-Einheit selber auch um eine potenziell vulkanisierbare Gruppe handelt, wurden Strategien zur Funktionalisierung der Abgangsgruppe erarbeitet um so ein $\alpha, \omega$-funktionalisiertes Polymer zu erhalten. Es wurden verschiedene Verbindungen synthetisiert, die funktionelle Gruppen tragen und sich für die Anbindung an RAFT-Agenzien eignen. Zwei dieser sog. Anker konnten erfolgreich für die Synthese von RAFT-Agenzien eingesetzt werden, die eine endständige Thioacetat-Funktion tragen. Neben einem Makro-RAFT-Agens, welches eine mehrstufige Synthese erfordert und daher für den Einsatz im großtechnischen Maßstab nur bedingt geeignet ist, konnte ein symmetrisches Trithiocarbonat synthetisiert werden, das an beiden Polymerkettenenden eine potenziell vulkanisierbare Gruppe trägt. Aufgrund der schlecht stabilisierten Abgangsgruppe kommt dieses Trithiocarbonat jedoch nicht für die Anwendung in einer Butadien-Polymerisation in Frage. 
Darüber hinaus konnten zwei Monomere erfolgreich synthetisiert werden, die wegen ihrer styrolähnlichen Struktur und der Ähnlichkeit der Monomere Styrol und Butadien in ihrem Polymerisationsverhalten gut mit Butadien copolymerisieren und dabei die vulkanisationsaktive Gruppe entlang der Polymerkette einbauen. Das Hauptaugenmerk weiterer Untersuchungen lag dabei auf den Copolymerisationseigenschaften von 4-Vinylbenzylthioacetat (ViBET), da ViBET unerwartet in die radikalische Polymerisation eingreift. Dazu wurden umfangreiche Untersuchungen mit Styrol als Modellsystem für Butadien durchgeführt und die gewonnenen Erkenntnisse mit einer ButadienViBET-Copolymerisation verifiziert. Eine konzentrationsabhängige Inhibierung der radikalischen Polymerisation, die wahrscheinlich durch Radikalübertragungsreaktionen ausgelöst wird, verbunden mit vergleichsweise niedrigen Polymermolmassen führen zu einer engeschränkten Verwendbarkeit dieses Monomers in dem angestrebten Konzentrationsbereich von 2 - 5 mol-\%. Erste Vernetzungen während der Copolymerisation mit Styrol bestätigen die erfolgreiche Funktionalisierung und die Vulkanisationsaktivität des Comonomers. Die Beeinflussung der Polymerisation hinsichtlich der Bildung niedrigdisperser Copolymere sowie die gute Verfügbarkeit machen es außerdem zu einem Monomer mit interessanten Eigenschaften.

Das zweite Monomer, 4-Vinylbenzylbenzolthiosulfonat (ViBSuT), konnte nach umfangreicher Syntheseoptimierung in sehr guten Ausbeuten hergestellt werden. Es vernetzt (höchstwahrscheinlich aufgrund eines schwer zu beseitigenden, bifunktionellen Nebenprodukts) bereits während der Polymerisation und führt so zu einem sehr uneinheitlichen Polymer, in dem sich das funktionalisierte Monomer stark anreichert. So kann bereits mit niedrigen Comonomerkonzentrationen ein sehr gutes Ergebnise hinsichtlich Funktionalisierungsgrad erreicht werden.

Es konnte weiterhin gezeigt werden, dass Poly(butadien)-block-poly(vinylacetat) in einer radikalischen Polymerisation mit schaltbaren RAFT-Agenzien hergestellt werden kann, obwohl es sich um zwei Monomere mit extrem unterschiedlichen elektronischen Eigenschaften handelt, die in einer konventionellen radikalischen Polymerisation kein Copolymerisat bilden würden. Aufgrund der Ähnlichkeit der Monomere Vinylacetat und Ethylen eröffnet dies neue Wege in der kontrollierten radikalischen Polymerisation von Poly(butadien)-block-poly(ethylen) und erweitert so das Spektrum der unpolaren Monomere in der RAFT-Polymerisation zur Herstellung von Synthesekautschuken. 


\section{Inhaltsverzeichnis}

1 Einleitung 1

2 Theorie 5

2.1 Radikalische Polymerisation . . . . . . . . . . . . . . 6

2.1 .1 Initiierung . . . . . . . . . . . . . . 6

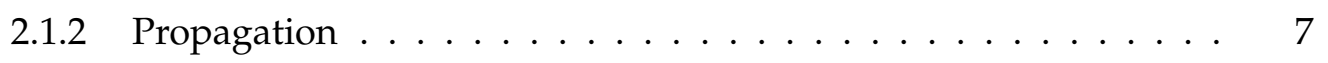

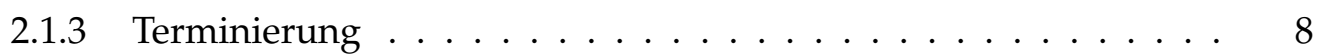

2.1 .4 Kinetik . . . . . . . . . . . . . . . . 8

2.2 Molmassenverteilung . . . . . . . . . . . . . . . . . . . 10

2.2.1 Zahlenmittel . . . . . . . . . . . . . . . . . 11

2.2.2 Massenmittel . . . . . . . . . . . . . . . . . . 11

2.2 .3 Peakmolmasse . . . . . . . . . . . . . . . . . . 11

2.2 .4 Dispersität . . . . . . . . . . . . . . . . . 12

2.2.5 Größenausschlusschromatographie . . . . . . . . . . . 12

2.3 Copolymerisation . . . . . . . . . . . . . . . . . . 14

2.4 Kontrollierte radikalische Polymerisation . . . . . . . . . . . . . . 17

2.4 .1 Terminologie . . . . . . . . . . . . . . . . . . 18

2.4.2 Nitroxidvermittelte Polymerisation, NMP . . . . . . . . . . . . . 19

2.4.3 Atomtransfer-radikalische Polymerisation, ATRP . . . . . . . . . 19

2.4.4 Reversibler Additions-Fragmentierungs-Kettentransfer, RAFT . . 20

2.5 Elastomere . . . . . . . . . . . . . . . . . . . . . . . . . 27

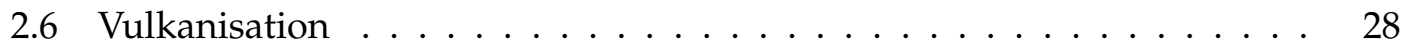

2.6 .1 Additive . . . . . . . . . . . . . . . . . . . . 29

2.7 Auswahl der Monomere . . . . . . . . . . . . . . . . . . . . 30

2.7.1 Copolymerisationsparameter . . . . . . . . . . . . . . 31 
3 Zielsetzung 33

4 Entwicklung und Synthese funktionalisierter RAFT-Agenzien 37

4.1 Forschungsstand . . . . . . . . . . . . . . 38

4.1 .1 Dithiobenzoate . . . . . . . . . . . . . . 38

4.1 .2 Trithiocarbonate . . . . . . . . . . . . . . . 41

4.1.3 Fazit und Konsequenzen für die Synthese neuer RAFT-Agenzien 45

4.2 Synthese funktionalisierter Anker . . . . . . . . . . . . . . . . . . . . 46

4.3 Trithiocarbonat mit Thioacetat-Funktionalität . . . . . . . . . . . . . . 48

4.4 Makro-RAFT-Agens mit Thioacetat-Funktionalität . . . . . . . . . . . . . 48

5 Entwicklung und Synthese funktionalisierter Monomere 51

5.1 Copolymerisationsverhalten . . . . . . . . . . . . . . . . 52

5.2 Nukleophile Substitution . . . . . . . . . . . . . . . . . . . . . . 54

5.2.1 Synthese von 4 -Vinylbenzylthioacetat $($ ViBET $) \ldots \ldots . . . .54$

5.3 Phasentransferkatalyse . . . . . . . . . . . . . . . 55

5.3.1 Synthese von 4-Vinylbenzylbenzolthiosulfonat (ViBSuT) . . . . 57

6 RAFT-Polymerisationen $\quad 61$

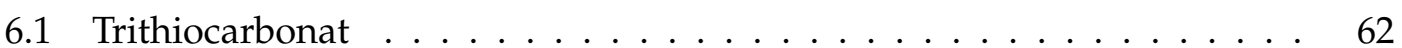

6.2 Makro-RAFT-Agens . . . . . . . . . . . . . . . . . . 65

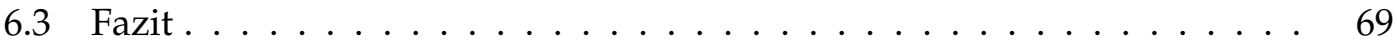

7 Copolymere mit 4-Vinylbenzylthioacetat $\quad 71$

7.1 Einfluss auf die Copolymerisation . . . . . . . . . . . . . . 72

7.2 Konzentrationsabhängiges Verhalten . . . . . . . . . . . . . . . . . 74

7.3 Polymerisationen mit ViBET-Derivaten . . . . . . . . . . . . 78

7.4 ESR-Spektroskopie . . . . . . . . . . . . . . . . . . . . . 84

7.5 Styrol-Polymerisation mit Inhibitor . . . . . . . . . . . . . . . . . . 85

7.6 Untersuchung der 4-Vinylbenzyl-Abgangsgruppe . . . . . . . . . . . 88

7.7 Styrol-ViBET-Copolymerisationen . . . . . . . . . . . . . . . . 92

7.7.1 Polymerzusammensetzung . . . . . . . . . . . . . . . 97

7.8 Butadien-ViBET-Copolymerisationen . . . . . . . . . . . . . . . 98

7.9 Fazit . . . . . . . . . . . . . . . . . . . . . . . . . . . . . . 101

8 Copolymere mit 4-Vinylbenzylbenzolthiosulfonat 103

8.1 Einfluss auf die Copolymerisation . . . . . . . . . . . . . . . . . . 104

8.2 Fazit . . . . . . . . . . . . . . . . . . . . . . . . 106 
9 Schaltbare RAFT-Agenzien $\quad 107$

9.1 Reagenzien . . . . . . . . . . . . . . . . . . . . . . . . . 108

9.2 MAM-Polymerisation . . . . . . . . . . . . . . . . . . . . . . . 109

9.2.1 Styrol-Polymerisation . . . . . . . . . . . . . . . . . . 109

9.2.2 Butadien-Polymerisation . . . . . . . . . . . . . . . . . . 110

9.3 LAM-Polymerisation . . . . . . . . . . . . . . . . . . . . . . . . 113

9.3.1 Vinylacetat-Polymerisation mit Styrol-Makro-RAFT-Agens . . . . 113

9.3.2 Vinylacetat-Polymerisation mit Butadien-Makro-RAFT-Agens . . 116

9.4 Fazit . . . . . . . . . . . . . . . . . . . . . . . . . . . 118

10 Ausblick 119

11 Experimentalteil $\quad 121$

11.1 Chemikalien . . . . . . . . . . . . . . . . . . . . . . . . . . . . . 121

11.2 Monomere . . . . . . . . . . . . . . . . . . . . . . 122

11.3 Initiatoren . . . . . . . . . . . . . . . . . . . . 122

11.4 RAFT-Agenzien . . . . . . . . . . . . . . . . . . . . . 122

11.5 Verwendete Geräte . . . . . . . . . . . . . . . . . . . . . . . . . 123

11.6 Synthesen . . . . . . . . . . . . . . . . . . . . . . . . . 124

11.7 Nummerierung . . . . . . . . . . . . . . . . . . . . . . 132

11.8 Polymerisationen . . . . . . . . . . . . . . . . . . . . 132

11.9 Aufbau des Hochdruck-Laborautoklaven . . . . . . . . . . . . . . . . 133

11.9.1 Zubehör des experimentellen Aufbaus . . . . . . . . . . . . . . 134

11.9.2 Versuchsdurchführung . . . . . . . . . . . . . . . . . . . . . 137

$\begin{array}{lr}\text { Abkürzungen } & 141\end{array}$

$\begin{array}{ll}\text { Literaturverzeichnis } & 145\end{array}$

$\begin{array}{ll}\text { Danksagung } & 155\end{array}$ 



\section{1 \\ Einleitung}

Aus dem heutigen Alltag sind synthetische Polymere, umgangssprachlich auch als Kunststoffe oder Plastik bezeichnet, nicht mehr wegzudenken. Sie spielen eine große Rolle in der Verpackungsindustrie, erlangen zunehmend Bedeutung im Automobilbau, in Lackfarben, als Klebstoffe, bei Funktionsmaterialen (z. B. für Funktionsbekleidung) und in unzähligen weiteren Bereichen. Dementsprechend groß ist ihre wirtschaftliche Bedeutung. In Deutschland wurden im Jahr 2011 13,5 Mio. Tonnen Kunststoff verarbeitet, davon 2,8 Mio. Tonnen im Bereich technische Teile (Fahrzeuge, Elektronik und Maschinenbau) und insgesamt ein Umsatz von 55,9 Mrd. Euro erwirtschaftet. ${ }^{[1]}$ Die Kunststofferzeugung macht einen Umsatz von weiteren ca. 40 Mrd. Euro aus. ${ }^{[2]}$

Bei den natürlichen Polymeren spielt der Naturkautschuk eine wichtige Rolle. Ausgezeichnete mechanische und dynamische Eigenschaften sowie eine gute Verfügbarkeit bei akzeptablen Preisen führten dazu, dass der Naturkautschuk bis heute ein begehrter Rohstoff ist und vor allem von der Reifenindustrie in großen Mengen verarbeitet wird. So wurden im Jahr 2012 weltweit 11 Mio. Tonnen Naturkautschuk produziert, denen 15 Mio. Tonnen Synthesekautschuk gegenüberstehen. ${ }^{[3]}$ Knapp $43 \%$ der weltweiten Kautschukproduktion ist somit natürlichen Ursprungs. Circa die Hälfte des Kautschuks wird in der Reifenindustrie verarbeitet, ${ }^{[4,5]}$ der Rest findet im Automobilbau, in der Thermoplastmodifikation, als Isolierung von Kabeln und Leitungen, im Maschi- 
nenbau, beim Bau und in sonstigen Bereichen Anwendung. ${ }^{4]}$ Neben Rohstoffpreisen und Energiekosten hat die Entwicklung der Fahrzeugindustrie im Allgemeinen einen großen Einfluss auf die Kautschukindustrie.

Die synthetischen Polymere sind ein relativ junges Fachgebiet. Das erste vollständig synthetisch hergestellte Polymer ist das Bakelit ${ }^{\circledR}$, welches zu Beginn des 20. Jahrhunderts von Leo Hendrik Baekeland (belgischer Chemiker) entwickelt wurde, der auf der Suche nach einem Isolator für Stromleitungen diesen duroplastischen Werkstoff entdeckte. Es handelt sich dabei um ein Phenol-Formaldehyd-Harz, das er sich im Jahr 1907 in den USA patentieren ließ ${ }^{[6]}$ und das ab dem Jahr 1910 von der Bakelite GmbH technisch hergestellt wurde. ${ }^{\text {[7] }}$

Polymere natürlichen Ursprungs waren jedoch schon früher bekannt. Bereits im Jahr 1806 führte John Gough, ein amerikanischer Naturphilosoph, an Naturkautschuk Experimente zur Gummielastizität durch. Im Jahr 1839 entdeckte Charles Goodyear die Möglichkeit, Naturkautschuk zu vulkanisieren. Kautschuk, ein weiches, klebriges Naturprodukt, wird dadurch ein unschätzbar wertvoller Rohstoff mit gummielastischen Eigenschaften. Er wird aus Pflanzen gewonnen, welche im sogenannten Kautschukgürtel (eine Zone zwischen $15^{\circ}$ nördlicher und südlicher Breite) gedeihen. ${ }^{[8]}$ Hauptlieferant ist der Kautschukbaum Hevea Brasiliensis, der ursprünglich in Brasilien beheimatet ist. Die Latexmilch, der Saft der kautschukproduzierenden Pflanzen, ist eine Dispersion von Kautschukpolymerpartikeln in Wasser. Der Kautschukanteil liegt je nach Pflanzenart bei bis zu $40 \%$. Neben Kautschuk sind geringe Mengen Proteine, Harze und Mineralstoffe im Latex gelöst bzw. dispergiert. Koagulation durch Zugabe von Säure führt zum Ausflocken des Kautschuks, welcher anschließend zu Fellen verpresst, gewaschen und zwecks Konservierung nachbehandelt wird. ${ }^{[5,8]}$

Während der Kautschuk des Hevea Brasiliensis reines cis-1,4-Polyisopren enthält, produzieren z. B. der Balatabaum Manilkara bidentata und der Guttaperchabaum Palaquium gutta reines trans-1,4-Polyisopren, welches als Balata bzw. Gutta(percha) bezeichnet wird. Die cis- und die trans-Variante unterscheiden sich in den physikalischen Eigenschaften. Beide sind auch synthetisch herstellbar, weisen dann aber eine geringere Stereoselektivität als die Polyisoprene natürlichen Ursprungs auf. Polyisopren befindet sich genauso wie Polybutadien, welches in Synthesekautschuk Verwendung findet, bei Raumtemperatur oberhalb der Glasübergangstemperatur im gummielastischen Zustand, in dem eine Weiterverarbeitung nur schwer möglich ist. Goodyear ließ eine Schwefel-Kautschuk-Mischung auf eine heiße Herdplatte fallen und das Ergebnis war eine trockene, elastische Substanz: Die Geburtsstunde der Vulkanisation. Schwefel und andere Additive sorgen für eine Vernetzung des Kautschuks, was sich auf dessen phy- 
sikalische Eigenschaften auswirkt.

Die Bedeutung des Kautschuks stieg rasant, erfüllte er doch die Voraussetzungen für viele technische Anwendungen. Die Verwendung von Naturkautschuk setzte aber den uneingeschränkten Zugang zu den Kautschukplantagen in Übersee (Brasilien und Asien) voraus, der ab Beginn des Ersten Weltkrieges nicht mehr gewährleistet war.

Nachdem mit Bakelit seit wenigen Jahren das erste vollsynthetische Polymer bekannt war, begann in Deutschland während der beiden Weltkriege verstärkt auch die Suche nach einer synthetischen Alternative zu dem Naturprodukt Kautschuk. In den Farbfabriken Bayer ${ }^{\circledR}$ in Leverkusen wurde während des Ersten Weltkriegs Methylkautschuk (Poly-2,3-Dimethylbutadien) als Gummiersatz produziert, der allerdings nach Kriegsende keine weitere Verwendung fand. Im Zweiten Weltkrieg produzierte der Chemiekonzern I. G. Farben in Schkopau Styrol-Butadien-Kautschuk, der unter dem Namen Buna bekannt wurde. Hierbei handelt es sich um ein Copolymerisat aus 1,3-Butadien und Styrol („Buna $\mathrm{S}^{\prime \prime}$ ) bzw. Acrylnitril („Buna N“), das in einer anionischen Polymerisation mit Natrium hergestellt wird. ${ }^{[9,10]}$

Heutzutage nimmt bei synthetisch hergestellten Polymeren die radikalische Polymerisation einen immer größeren Stellenwert ein: Rund die Hälfte aller synthetischen Polymere wird inzwischen radikalisch polymerisiert. Radikalische Polymerisationen sind robust und vielseitig; außer Sauerstoff tolerieren sie nahezu jede Verunreinigung, es ist eine breite Palette an Monomeren nutzbar, die Polymerisation kann bei Atmosphärendruck und Temperaturen zwischen Zimmertemperatur und $100^{\circ} \mathrm{C}$ erfolgen, es gibt eine Vielzahl an Initiatoren, die thermisch, photochemisch oder chemisch das Kettenwachstum initiieren können. Die Polymerisation kann in Substanz, in wässriger Suspension, in Emulsion oder in Lösung durchgeführt werden, ganz nach Bedarf. [11]

Im Gegensatz zu ionischen Polymerisationen kann bei einer radikalischen Polymerisation das Wachstum einer Kette mittels Terminierung beendet werden, was bei einem anionischen oder kationischen aktiven Zentrum nicht möglich ist; dort gibt es nur die Terminierung durch Verunreinigungen wie Wasser und Kohlenstoffdioxid. Dies kann durch sehr geringe Radikalkonzentrationen unterdrückt, aber nicht vollständig unterbunden werden. Forschungen haben in den vergangenen 30 Jahren zu Polymerisationssystemen geführt, welche ein stärkeres Eingreifen und Steuern des Polymerisationsprozesses ermöglichen. Die Zugabe von Additiven kann ebenfalls die Produkteigenschaften maßgeblich beeinflussen und zu funktionellen Polymermaterialien führen. 



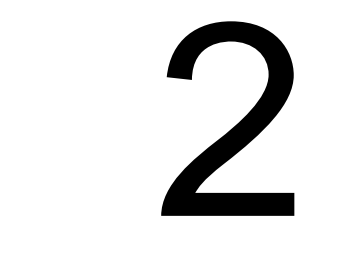

Theorie

Die Polyreaktionen werden in zwei große Klassen unterteilt, die Stufen- und die Kettenwachstumsreaktionen. Bei der Stufenwachstumsreaktion wird außerdem unterschieden zwischen Polyaddition und Polykondensation. Die Kettenwachstumsreaktion wird häufig als Polymerisation bezeichnet.

Bei Stufenwachstumreaktionen erfolgt sowohl die Reaktion von Monomermolekülen miteinander als auch die Reaktion von Monomer mit Oligomer oder von Oligomeren untereinander. Bei der Kettenwachstumreaktion ist dagegen immer mindestens ein Monomermolekül am Wachstumsschritt beteiligt.

Bei einem Polymer, dass in einer Polyaddition hergestellt worden ist, sind die einzelnen Monomereinheiten noch deutlich zu erkennen, da oft lediglich ein Proton verschoben wird um die Bindung einzugehen. Ein typisches Beispiel für eine Polyaddition ist die Reaktion von Diisocyanaten mit Diolen zu Polyurethanen (diese finden Verwendung z. B. als Bauschaum und in Matratzen). Bei der Polykondensation erfolgt eine Addition unter Abspaltung eines kleinen Moleküls wie z. B. Wasser bei der Veresterung von Dicarbonsäuren mit Diolen zu Polyestern, die u. a. als Textilfasern oder für Getränkeflaschen verwendet werden.

Bei Kettenwachstumspolymerisationen, die ionisch oder radikalisch möglich sind, wächst eine Kette mit einem aktiven Zentrum. An dieses Zentrum werden weitere 
Monomereinheiten angelagert. Bei ionischen Polymerisationen kann dieses Wachstum erfolgen bis entweder das ganze Monomer aufgebraucht ist oder aber bis es zu einem Abbruch durch eine Verunreinigung wie Sauerstoff, Wasser oder Kohlenstoffdioxid kommt. Radikalische Polymerisationen können durch Terminierung zweier Radikale zum Erliegen kommen, wobei hier zwischen Disproportionierung und Kombination unterschieden werden muss. In dieser Arbeit werden ausschließlich radikalischinitiierte Kettenwachstumsreaktionen durchgeführt, daher wird auf die anderen Möglichkeiten nicht näher eingegangen.

\subsection{Radikalische Polymerisation}

Die radikalsche Polymerisation ist eine sehr verbreitete Technik zur Synthese hochmolekularer Polymere. Sie zeichnet sich dadurch aus, dass eine große Bandbreite an Monomeren verwendet werden kann, eine große Zahl funktioneller Gruppen toleriert wird und sie einfach und kostengünstig in der Anwendung ist. ${ }^{[12]}$ Einer idealen radikalischen Polymerisation liegen folgende Annahmen zugrunde:

- Alle Reaktionen verlaufen irreversibel.

- Alle Radikale haben die gleiche Reaktivität, unabhängig von der Länge der Polymerkette.

- Monomere werden nur während des Wachstumsschrittes verbraucht.

- Terminierung erfolgt ausschließlich durch Kombination oder Disproportionierung.

Als Monomere eignen sich Verbindungen mit terminalen Doppelbindungen wie Ethylen, 1,3-Butadien, Vinylchlorid, Styrol oder (Meth-)Acrylate.

\subsubsection{Initiierung}

Die Initiierung erfolgt bei einer radikalischen Polymerisation durch den thermischen oder photochemischen Zerfall eines Initiators (z. B. Azoverbindungen oder Peroxide) oder durch Reduktion von Wasserstoffperoxid mit mehrwertigen Metallionen. ${ }^{[9]}$ In dieser Arbeit wurden ausschließlich Azoverbindungen verwendet, die thermisch initiiert wurden. Unter Stickstoffabspaltung bilden sich zwei kohlenstoffzentrierte Ra- 
dikale (siehe Schema 2.1), die die Polymerisation starten können. Nebenbei kann es zu ungewünschten Nebenreaktionen wie einer Radikalrekombination aufgrund der räumlichen Nähe der beiden gebildeten Initiatorradikale („Käfigeffekt“) kommen.

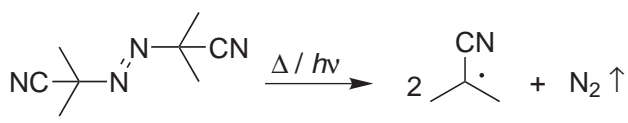

Schema 2.1 Azoinitiatorzerfall am Beispiel 2,2'-Azo-bis(isobutyronitril) (AIBN).

\subsubsection{Propagation}

Bei der Propagation lagert sich ein Radikal an ein Monomermolekül an. Dabei bildet sich ein neues Radikal, bei dem das ungepaarte Elektron an einem der Kohlenstoffatome der ehemaligen Doppelbindung lokalisiert ist. Die Anlagerung des ersten Monomermoleküls an das Initiatorradikal wird Kettenstart genannt, die Anlagerungen von weiteren Monomermolekülen Kettenwachstum. Durch die terminale Doppelbindung gibt es sowohl beim initialen als auch bei allen weiteren Wachstumsschritten 2 Möglichkeiten, wie sich das Monomer anlagern kann. Die bevorzugte Möglichkeit ist am Beispiel AIBN/Styrol in Schema 2.2 dargestellt. Diese sog. Anti-Markovnikov-Addition ist sowohl sterisch als auch aufgrund geringerer Aktivierungsenergie wahrscheinlicher. ${ }^{[9]}$ Die Bildung eines terminalen $\mathrm{CH}_{2}$-Radikals, das an einem primären Kohlenstoffatom zentriert wäre, ist weniger wahrscheinlich.

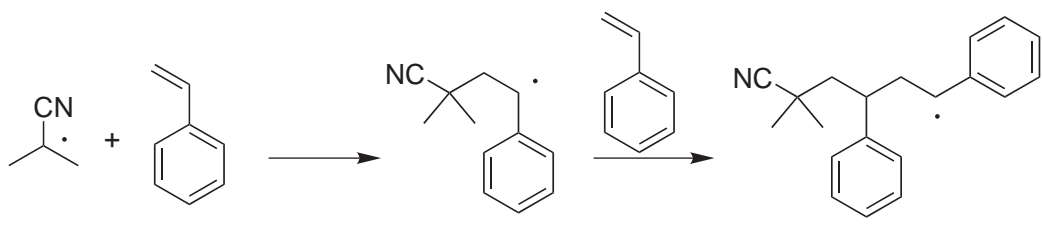

Schema 2.2 Kettenstart und Kettenwachstum am Beispiel von AIBN mit Styrol. 


\subsubsection{Terminierung}

Die Terminierung kann nach zwei möglichen Mechnismen erfolgen. Bei der Rekombination reagieren zwei radikaltragende Spezies miteinander und bilden "totes Polymer", dessen Molmasse sich aus der Addition der Massen der beiden Radikalketten ergibt. Bei der Disproportionierung hingegen abstrahiert ein Makroradikal ein Wasserstoffatom oder ein anderes Atom einer anderen wachsenden Kette. Es bilden sich eine gesättigte sowie eine ungesättigte Endgruppe unter Erhaltung der jeweiligen Molmasse.

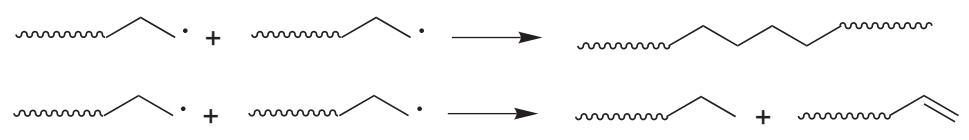

Schema 2.3 Terminierung mittels Kombination (oben) bzw. Disproportionierung (unten).

\subsubsection{Kinetik}

Schema 2.4 zeigt ein vereinfachtes kinetisches Schema einer radikalischen Polymerisation. Neben den oben angesprochenen Schritten Initiierung, Kettenstart, Propagation und Terminierung ist die Radikalübertragung dargestellt. Sie wird bei idealen Betrachtungen meist vernachlässigt, ist aber häufig präsent oder wird gezielt angestrebt, z. B. durch Zugabe von Thiolen. Als Überträger dienen neben Verunreinigungen auch Lösungsmittel- oder Initiatormoleküle sowie tote Polymerketten. ${ }^{[13,14]}$

$\begin{array}{ll}\text { Initiierung: } & \quad \mathrm{In} \stackrel{k_{\mathrm{d}}}{\longrightarrow} 2 \mathrm{I} \cdot \\ \text { Kettenstart: } & \mathrm{I} \cdot+\mathrm{M} \stackrel{k_{\mathrm{S}}}{\longrightarrow} \mathrm{P}_{1} \cdot \\ \text { Propagation: } & \mathrm{P}_{m} \cdot+\mathrm{M} \stackrel{k_{\mathrm{p}}}{\longrightarrow} \mathrm{P}_{m+1} \cdot \\ \text { Rekombination: } & \mathrm{P}_{n} \cdot+\mathrm{P}_{m} \cdot \stackrel{k_{\mathrm{t}, \mathrm{k}}}{\longrightarrow} \mathrm{P}_{n+m} \\ \text { Disproportionierung: } & \mathrm{P}_{n} \cdot+\mathrm{P}_{m} \cdot \stackrel{k_{\mathrm{t}, \mathrm{d}}}{\longrightarrow} \mathrm{P}_{n}+\mathrm{P}_{m} \\ \text { Radikalübertragung: } & \mathrm{P}_{n} \cdot+\mathrm{X} \stackrel{k_{\mathrm{tr}}}{\longrightarrow} \mathrm{P}_{n}+\mathrm{X} .\end{array}$

Schema 2.4 Vereinfachtes kinetisches Schema radikalischer Polymerisationen. 
Bei der Initiierung handelt es sich um den geschwindigkeitsbestimmenden Schritt, da sie deutlich langsamer als die Startreaktion verläuft. Bezogen auf die Initiatorkonzentration ist die Reaktionsgeschwindigkeit 1 . Ordnung.

$$
\frac{\mathrm{d}[\mathrm{I} \cdot]}{\mathrm{d} t}=2 \cdot k_{\mathrm{d}} \cdot f \cdot[\operatorname{In}]
$$

[I-] ist die Konzentration des Initiatorradikals, $k_{\mathrm{d}}$ der Geschwindigkeitskoeffizient der Zerfallsreaktion, $f$ die Initiatoreffizienz und [In] die Initiatorkonzentration. Der Faktor 2 ergibt sich aus der Zerfallsreaktion, bei der zwei Radikale aus einem Initiatormolekül gebildet werden. Bei der Initiatoreffizienz handelt es sich um einen Korrekturfaktor, der berücksichtigt, dass nicht jedes entstehende Radikal an der Startreaktion beteiligt ist, da es vorher mit einem anderen Radikal kombinieren kann (siehe oben, Käfigeffekt). $f$ kann somit Werte zwischen 0 (kein Kettenstart) und 1 (jedes gebildete Radikal startet eine Kette) annehmen. Die Startreaktion wird hier vernachlässigt, da sie für die Kinetik unbedeutend ist. Die Monomerabnahme während der Propagation wird durch Gleichung 2.2 beschrieben (Monomerkonzentration [M], Radikalkonzentration $\left[\mathrm{P}_{m} \cdot\right]$, Geschwindigkeitskoeffizient $k_{\mathrm{p}}$ ):

$$
-\frac{\mathrm{d}[\mathrm{M}]}{\mathrm{d} t}=k_{\mathrm{p}} \cdot[\mathrm{M}] \cdot\left[\mathrm{P}_{\mathrm{m}} \cdot\right]
$$

Bei radikalischen Polymerisationen unter realen Bedingungen kommt es zu Radikaltransfer. Die Übertragungskonstante $C_{\text {tr }}$ ist definiert als Quotient aus $k_{\text {tr }}$ und $k_{\mathrm{p}}$. Bei einer Radikalübertragung bleibt die Radikalkonzentration - im Gegensatz zu Kombinations- oder Disproportionierungsreaktionen - unverändert, während der Polymerisationsgrad sinkt. Diese Transferreaktion lässt sich durch folgendes Geschwindigkeitsgesetz beschreiben (Transfer durch das Molekül X mit der Konzentration [X], Geschwindigkeitskoeffizient $k_{\mathrm{tr}}$ ):

$$
\begin{array}{r}
-\frac{\mathrm{d}[\mathrm{X}]}{\mathrm{d} t}=k_{\mathrm{tr}} \cdot[\mathrm{P} \cdot] \cdot[\mathrm{X}] \\
C_{\mathrm{tr}}=\frac{k_{\mathrm{tr}}}{k_{\mathrm{p}}}
\end{array}
$$

Es gibt zwei verschiedene Möglichkeiten des Kettenabbruchs, die sogenanntes „totes Polymer", welches kein Radikal mehr trägt, liefern: Dies ist zum einen die Kombination zweier Makroradikale zu einem Polymermolekül oder die Disproportionierung, bei der sich zwei Polymermoleküle aus zwei Makroradikalen bilden. Es handelt sich hierbei um einen bimolekularen Prozess, wie in Schema 2.3 dargestellt ist. Die kinetische Betrachtung geht davon aus, dass die Makroradikale $\mathrm{P}_{n}$ und $\mathrm{P}_{m}$ ununterscheidbar sind und dieselbe Reaktivität aufweisen. Sie werden daher als P. zusammengefasst. 
$k_{\mathrm{t}, \mathrm{k}}$ und $k_{\mathrm{t}, \mathrm{d}}$ addieren sich zu $k_{\mathrm{t}}$. Für die Abnahme der Makroradikalkonzentration ergibt sich:

$$
-\frac{\mathrm{d}[\mathrm{P} \cdot]}{\mathrm{d} t}=2 \cdot k_{\mathrm{t}} \cdot[\mathrm{P} \cdot]^{2}
$$

Um die Ordnung der Polymerisationsgeschwindigkeit zu bestimmen, werden die Radikalbildung beim Initiatorzerfall (Gleichung 2.1) und der Radikalverbrauch durch Terminierung (Gleichung 2.5) gleichgesetzt.

$$
\begin{array}{r}
\frac{\mathrm{d}[\mathrm{I} \cdot]}{\mathrm{d} t}=-\frac{\mathrm{d}[\mathrm{P} \cdot]}{\mathrm{d} t} \\
2 \cdot k_{\mathrm{d}} \cdot f \cdot[\mathrm{In}]=2 \cdot k_{\mathrm{t}} \cdot[\mathrm{P} \cdot]^{2} \\
\Rightarrow[\mathrm{P} \cdot]=\sqrt{\frac{k_{\mathrm{d}} \cdot f \cdot[\mathrm{In}]}{k_{\mathrm{t}}}}
\end{array}
$$

Mit Hilfe dieser Gleichungen lässt sich die Abhängigkeit der Polymerisationsgeschwindigkeit von der Monomerkonzentration herleiten. Dies geschieht unter der Annahme, dass $k_{\mathrm{d}}$ klein ist und die Konzentration der Initiatorradikale während der Reaktion annähernd konstant ist:

$$
-\frac{\mathrm{d}[\mathrm{M}]}{\mathrm{d} t}=k_{\mathrm{p}} \cdot[\mathrm{M}] \cdot[\mathrm{P} \cdot]=k_{\mathrm{p}} \cdot \sqrt{\frac{k_{\mathrm{d}}}{k_{\mathrm{t}}} \cdot f \cdot[\mathrm{In}]} \cdot[\mathrm{M}]
$$

Bezüglich der Monomerkonzentration [M] ist die Polymerisationsgeschwindigkeit somit 1. Ordnung.

\subsection{Molmassenverteilung}

Die Molmasse ist ein wichtiges Charakteristikum für Makromoleküle und kann abhängig von der Kettenlänge mehrere Hunderttausend g/mol betragen. Die Besonderheit bei Polymeren ist die Uneinheitlichkeit der Moleküle hinsichtlich ihrer Masse. Weder bei den meisten natürlichen Makromolekülen (wie z. B. Naturkautschuk oder Cellulose), noch bei synthetischen Makromolekülen (Synthesekautschuk o.ä.) ist die Molmasse aller Moleküle gleich. Eine Ausnahme bilden z. B. die Proteine. Daraus resultiert eine Molmassenverteilung, auch Molekulargewichtsverteilung genannt. Es gibt unterschiedliche Möglichkeiten, die Molmasse für ein Polymer zu beschreiben. Die verbreitetsten werden im Folgenden kurz vorgestellt. 


\subsubsection{Zahlenmittel}

Das Zahlenmittel $\bar{M}_{\mathrm{n}}$ setzt die unterschiedlichen auftretenden Molmassen in Bezug zu der Anzahl der Ketten, welche die einzelnen Molmassen besitzen.

$$
\bar{M}_{\mathrm{n}}=\sum x_{i} M_{i}
$$

Unter Einbeziehen der Definition des Molenbruchs $x_{\mathrm{i}}\left(N_{\mathrm{i}}\right.$ sei die Anzahl der Moleküle mit der Länge $i$ und $N$ die Gesamtzahl der Moleküle)

$$
x_{i}=\frac{N_{i}}{N}
$$

folgt für das Zahlenmittel:

$$
\bar{M}_{\mathrm{n}}=\frac{\sum N_{i} M_{i}}{\sum N_{i}}
$$

Wenn nicht anders angegeben, bezieht sich der Ausdruck „mittlere Molmasse“ in der Diskussion der Ergebnisse immer auf das Zahlenmittel.

\subsubsection{Massenmittel}

Im Gegensatz zum Zahlenmittel ist beim Massenmittel $\bar{M}_{\mathrm{w}}$ die Masse der Bezugspunkt. Der Massenbruch $w_{i}$ (auch Gewichtsbruch genannt) entspricht dem Verhältnis der Masse $m_{i}$ aller Moleküle $N_{i}$ einer Länge $i$ zu der Gesamtmasse aller Moleküle im Polymer.

$$
w_{i}=\frac{N_{i} \cdot M_{i}}{\sum N_{i} M_{i}}
$$

Das Gewichtsmittel berechnet sich analog zu dem Zahlenmittel:

$$
\bar{M}_{\mathrm{w}}=\sum w_{i} M_{i}=\frac{\sum N_{i} M_{i}^{2}}{\sum N_{i} M_{i}}
$$

\subsubsection{Peakmolmasse}

Für einige Anwendungen ist die Molmasse des höchsten Peaks, $M_{\mathrm{p}}$, interessant. Dies ist die Molmasse, die absolut gesehen am häufigsten vorkommt und z. B. bei niedrigdispersen Polymerstandards angegeben wird. Darüber hinaus kann auf die Peakmolmasse zurückgegriffen werden, wenn $M_{\mathrm{n}}$ aufgrund von Überlagerungen nicht bestimmt werden kann. 


\subsubsection{Dispersität}

Die Dispersität $Đ$ (veraltet: Polydispersitätsindex, PDI) entspricht dem Quotienten aus Massen- und Zahlenmittel. Die Uneinheitlichkeit $U$ gibt die Abweichung vom Idealwert $Ð=1$ an. Die Dispersität ist somit eine Maßzahl für die Breite der Molmassenverteilung. Bei einer sogenannten reversibel deaktivierten Polymerisation (siehe 2.4.1) ist $Đ$ i.d. R. kleiner als 1,2. Nach idealer Kinetik ist bei Terminierung durch Disproportionierung $Ð \geq 1,5$ und bei Terminierung durch Kombination $Ð \geq 2,0 .{ }^{[15]}$

Je breiter die Verteilung, desto uneinheitlicher (hinsichtlich Kettenlänge) ist das Polymer und desto größer ist $Ð$. Formel 2.16 gilt für den Spezialfall, dass die Molmassenverteilung einer Poisson-Verteilung entspricht, ${ }^{[16]}$ dabei ist $\bar{P}_{\mathrm{n}}$ das Zahlenmittel des Polymerisationsgrades. Bedingung dafür ist, dass die Anzahl der wachsenden Ketten konstant ist und die Propagationsgeschwindigkeit kettenlängenunabhängig ist.

$$
\begin{array}{r}
Ð=\frac{M_{\mathrm{w}}}{M_{\mathrm{n}}}=U+1 \\
Đ=1+\frac{1}{\bar{P}_{\mathrm{n}}}
\end{array}
$$

\subsubsection{Größenausschlusschromatographie}

Es gibt unterschiedliche Möglichkeiten, eine Molmassenverteilung zu bestimmen. Weit verbreitet ist die Größenausschlusschromatographie, auch Gelpermeationschromatographie (GPC) genannt (Englisch: size exclusion chromatography, SEC). Die Polymere werden nach ihrem hydrodynamischen Volumen aufgetrennt, welches proportional zur Molmasse ist: ${ }^{[17]}$

$$
V_{\mathrm{h}}=[\eta] \cdot M
$$

$[\eta]$ ist die intrinsische Viskosität.

Das gelöste Polymer (Lösungsmittel z. B. THF) wird durch Säulen mit porösem Material (z. B. aufgequollenes, vernetztes Polymer) aufgetrennt. Es finden weder Adsorption noch Desorption statt, die Trennung beruht allein auf Diffusion. Zwischen den Gelpartikeln befindet sich das äußere Volumen $V_{0}$, außerdem gibt es ein sog. inneres Volumen, das Porenvolumen $V_{\mathrm{i}}$. Alle Moleküle, die für die Poren zu groß sind, deren hydrodynamisches Volumen also oberhalb der Ausschlussgrenze der Säule liegt, werden mit dem Elutionsvolumen $V_{\mathrm{e}}$ ohne Verzögerung eluiert.

$$
V_{\mathrm{e}}=V_{0}
$$


Bei sehr kleinen Molekülen, die in alle Poren hineinpassen, addiert sich das innere Volumen der Poren, die aufgrund des hydrodynamischen Volumens komplett zugänglich sind, zu dem äußeren Volumen.

$$
V_{\mathrm{e}}=V_{0}+V_{\mathrm{i}}
$$

Wenn für die Moleküle aufgrund ihres hydrodynamischen Volumens nur ein Teil der Poren zugänglich ist, wird zu dem äußeren Volumen nur ein Teil des inneren Volumens addiert. Der Proportionalitätsfaktor $K_{\mathrm{d}}$ wird Verteilungskoeffizient genannt und ist eine Stoffkonstante, die von verschiedenen Parametern (u. a. der Teilchengröße, der Porengröße, dem Lösungsmittel und der Verzweigung des Polymers) abhängt.

$$
V_{\mathrm{e}}=V_{0}+K_{\mathrm{d}} \cdot V_{\mathrm{i}}
$$

Elutionszeit und -volumen sind invers proportional zum hydrodynamischen Volumen des Polymers.

Die Charakterisierung erfolgt über unterschiedliche Detektoren, die den Brechungsindex (RI, englisch für „refraction index“) oder die UV-Absorption messen. Die GPC ist eine relative Messmethode, ${ }^{[18]}$ die eine Kalibrierung mit Polymerstandards bekannter Molmasse benötigt. Dabei werden Polymere mit einer niedrig dispersen Molmassenverteilung vermessen und das Retentionsvolumen am Peakmaximum bestimmt. An eine Auftragung von $\log (M)$ vs. $V_{\mathrm{e}}$ wird eine Funktion angepasst. So lässt sich für jedes Polymer derselben Art die Molmasse bei bekanntem Retentionsvolumen berechnen. Liegen keine Standards vor, kann über eine universelle Kalibration die Molmasse bestimmt werden. Zwei unterschiedliche Polymere, die im gleichen Lösungsmittel gelöst sind, besitzen bei gleicher Retentionszeit das gleiche hydrodynamische Volumen. ${ }^{[19,20]}$

$$
[\eta]_{1} \cdot M_{1}=[\eta]_{2} \cdot M_{2}
$$

Dabei indiziert 1 den Polymerstandard und 2 das unbekannte Polymer. Die instrinsische Viskosität wird durch die Mark-Houwink-Beziehung ${ }^{[21,22]}$ berechnet:

$$
[\eta]=K \cdot M^{a}
$$

In der Formel beschreibt $a$ eine strukturabhängige Größe und nimmt Werte zwischen 0 und 2 an. Dabei entspricht 0 einer Kugel und 2 einem starren Stab. ${ }^{[17]}$ Typische Werte für Polymere liegen zwischen 0 und 1 (ataktisches Styrol in Cyclohexan bei $313 \mathrm{~K}$ : $a=0,554$, Polyacrylsäure in $1 \mathrm{M}$ wässriger $\mathrm{NaCl}$ bei $298 \mathrm{~K}: a=0,90^{[9]}$ ) und hängen vom 
Polymer, dem Lösungsmittel und der Temperatur ab. Die Konstante $K$ ist ein Maß für die Kettenbeweglichkeit. Die Werte liegen z. B. bei $6,31 \cdot 10^{5} \mathrm{dl} / \mathrm{g}$ für Polyacrylamid in Wasser bei $303 \mathrm{~K}$ und bei $41,6 \cdot 10^{5} \mathrm{dl} / \mathrm{g}$ für ataktisches Polystyrol in Cyclohexan bei 313 K. ${ }^{[9]}$ Aus Gleichung 2.21 und Gleichung 2.22 ergibt sich für die Molmasse von Polymer 2:

$$
M_{2}=\left(\frac{K_{1} \cdot M_{1}^{\left(a_{1}+1\right)}}{K_{2}}\right)^{\frac{1}{a_{2}+1}}
$$

Die Mark-Houwink-Beziehung gilt ausschließlich für Polymere, die ausreichend lang sind. Vinylpolymere erfüllen diese Vorraussetzung ab einer Kettenlänge von 50 100 Monomereinheiten. ${ }^{[20]}$

\subsection{Copolymerisation}

Polymere, die aus unterschiedlichen Monomeren zusammengesetzt sind, werden als Copolymere bezeichnet. Ein Copolymer, das sich aus den Monomeren A und B zusammensetzt, kann $u$. a. folgendermaßen aufgebaut sein: ${ }^{[9,23]}$

- Statistische Copolymere: A und B werden ungeordnet („,statistisch“) in die Polymerkette eingebaut.

- Alternierende Copolymere: A und B werden abwechselnd (,,alternierend“) eingebaut.

- Gradientencopolymere: Entlang der Polymerkette verläuft ein Gradient von Homopolymer A zu Homopolymer B.

- Blockcopolymere: Die Polymerkette ist aus zwei oder mehr miteinander verknüpften Homopolymerblöcken aus A und B aufgebaut.

- Pfropfcopolymere: An der Hauptkette aus A wachsen kammartig Seitenarme, die aus B aufgebaut sind.

Im Folgenden liegt der Fokus auf statistischen und Blockcopolymeren. Bei Blockcopolymeren ist die Kinetik unverändert, da die Blöcke nacheinander hergestellt werden. Die Kinetik von Copolymerisationsreaktionen, bei der statistische Copolymere gebildet werden, unterscheidet sich von der Kinetik einer radikalischen Homopolymerisation. Ein Makroradikal mit einer endständigen Monomereinheit A kann im nächsten Wachstumsschritt mit Monomer A oder B reagieren und ein Makroradikal mit endständiger 
Monomereinheit B kann im nächsten Wachstumsschritt ebenfalls mit Monomer A oder $B$ reagieren. Es kann also jeweils das gleiche oder das andere Monomer angelagert werden. Somit gibt es insgesamt vier Geschwindigkeitskoeffizienten für die Propagation (vorrausgesetzt, dass nur das Kettenende die Propagationsgeschwindigkeit beeinflusst und weder die Länge noch die Art der Kette): $k_{11}, k_{12}, k_{21}, k_{22}$ (siehe Schema 2.5).

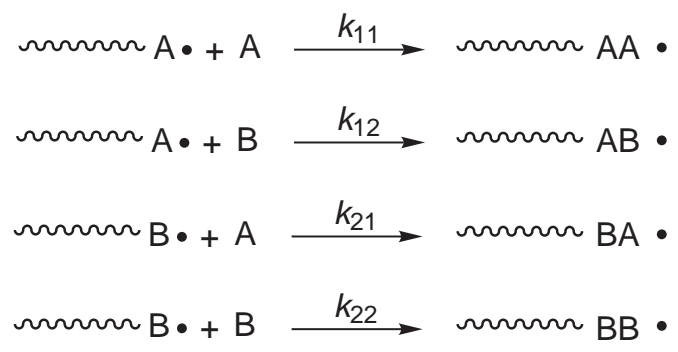

Schema 2.5 Copolymerisation der zwei Monomere A und B mit den Geschwindigkeitskoeffizienten $k$ : Kinetisches Schema. ${ }^{[23]}$

Für die Geschwindigkeit der Abnahme von Monomer A bzw. B ergibt sich:

$$
\begin{array}{r}
-\frac{\mathrm{d}[\mathrm{A}]}{\mathrm{d} t}=k_{11} \cdot[\mathrm{A}] \cdot \sum[\mathrm{A} \cdot]+k_{21} \cdot[\mathrm{A}] \cdot \sum[\mathrm{B} \cdot] \\
-\frac{\mathrm{d}[\mathrm{B}]}{\mathrm{d} t}=k_{12} \cdot[\mathrm{B}] \cdot \sum[\mathrm{A} \cdot]+k_{22} \cdot[\mathrm{B}] \cdot \sum[\mathrm{B} \cdot]
\end{array}
$$

Gerade bei statistischen Copolymerisationen ist es nützlich zu wissen, in welchem Verhältnis die beiden Monomere eingebaut werden. Dieses Einbauverhältnis entspricht dem Quotient der Gleichungen 2.24 und 2.25.

$$
\frac{\mathrm{d}[\mathrm{A}]}{\mathrm{d}[\mathrm{B}]}=\frac{[\mathrm{A}]}{[\mathrm{B}]} \cdot\left(\frac{k_{11} \cdot \frac{\sum[\mathrm{A} \cdot]}{\sum[\mathrm{B} \cdot]}+k_{21}}{k_{12} \cdot \frac{\sum[\mathrm{A} \cdot]}{\sum[\mathrm{B} \cdot]}+k_{22}}\right)
$$

Durch das Gleichgewicht zwischen der Zu- und Abnahme der Polymerketten mit Monomer A als Endgruppe bildet sich ein stationärer Zustand aus. Es gilt:

$$
\begin{array}{r}
k_{21} \cdot[\mathrm{A}] \cdot \sum[\mathrm{B} \cdot]=k_{12} \cdot[\mathrm{B}] \cdot \sum[\mathrm{A} \cdot] \\
\frac{\sum[\mathrm{A} \cdot]}{\sum[\mathrm{B} \cdot]}=\frac{k_{21} \cdot[\mathrm{A}]}{k_{12} \cdot[\mathrm{B}]}
\end{array}
$$

Gleichung 2.26 lässt sich nun unter Zuhilfenahme von Gleichungen 2.27 und 2.28 vereinfachen.

$$
\frac{\mathrm{d}[\mathrm{A}]}{\mathrm{d}[\mathrm{B}]}=\frac{[\mathrm{A}]}{[\mathrm{B}]} \cdot\left(\frac{\frac{k_{11}}{k_{12}} \cdot[\mathrm{A}]+[\mathrm{B}]}{\frac{k_{22}}{k_{21}} \cdot[\mathrm{B}]+[\mathrm{A}]}\right)
$$


Die Quotienten der Geschwindigkeitskoeffizienten $k_{11}$ und $k_{12}$ bzw. $k_{21}$ und $k_{22}$ werden auch als Reaktivitätsverhältnisse bezeichnet und sind für gängige Symsteme tabelliert. ${ }^{[24]}$

$$
r_{1}=\frac{k_{11}}{k_{12}} \quad(2.30) \quad r_{2}=\frac{k_{22}}{k_{21}}
$$

Hieraus resultiert nun die Copolymerisationsgleichung (Mayo-Lewis-Gleichung), welche die Zusammensetzung des Polymers aus den Monomeren A und B angibt.

$$
\frac{\mathrm{d}[\mathrm{A}]}{\mathrm{d}[\mathrm{B}]}=\frac{[\mathrm{A}]}{[\mathrm{B}]} \cdot\left(\frac{r_{1} \cdot[\mathrm{A}]+[\mathrm{B}]}{r_{2} \cdot[\mathrm{B}]+[\mathrm{A}]}\right)=\frac{1+\frac{[\mathrm{A}]}{[\mathrm{B}]} \cdot r_{1}}{1+\frac{[\mathrm{B}]}{[\mathrm{A}]} \cdot r_{2}}
$$

Die Copolymerisationsparameter $r_{1}$ und $r_{2}$ lassen Rückschlüsse auf den Polymerisationsmechanismus zu.

- $r=0 \quad$ Radikal lagert ausschließlich das Monomer an, das nicht Endgruppe ist

- $r<1$ Radikal lagert bevorzugt das Monomer an, das nicht Endgruppe ist

- $r=1 \quad$ Keine Bevorzugung eines Monomers

- $r>1$ Radikal lagert bevorzugt das Monomer an, das Endgruppe ist

- $r \rightarrow \infty$ Radikal lagert ausschließlich das Monomer an, das Endgruppe ist

Das Verhältnis der Änderungen der Monomerkonzentration spiegelt sich in der Copolymerzusammensetzung wieder. Für den differentiellen Monomerumsatz $U$ ergibt sich folgender Zusammenhang zwischen Monomer- und Copolymerzusammensetzung:

$$
\lim _{\Delta U \rightarrow 0} \frac{\mathrm{d}[\mathrm{A}]}{\mathrm{d}[\mathrm{B}]}=\frac{[\mathrm{A}(\mathrm{P})]}{[\mathrm{B}(\mathrm{P})]}
$$

Hierbei sei $[\mathrm{A}(\mathrm{P})]$ die Konzentration von Monomer A im Polymer P und $[\mathrm{B}(\mathrm{P})]$ die Konzentration von Monomer B im Polymer P. Zur Vereinfachung werden die Konzentrationsverhältnisse a bzw. $\mathrm{b}$ genannt und führen zu einer vereinfachten Darstellung der Copolymerisationsgleichung 2.32 .

$$
\begin{gathered}
b=\frac{1+a \cdot r_{1}}{1+\frac{r_{2}}{a}} \\
a=\frac{[\mathrm{A}]}{[\mathrm{B}]} \quad(2.35) \quad b=\frac{[\mathrm{A}(\mathrm{P})]}{[\mathrm{B}(\mathrm{P})]}
\end{gathered}
$$

Für unbekannte Copolymere können die Copolymerisationsparameter bestimmt werden, indem Copolymere mit niedrigem Umsatz (ideal nahe null) aus unterschiedli- 
chen Monomermischungen hergestellt werden. Das Polymer kann z. B. über NMRSpektroskopie oder Elementaranalyse hinsichtlich seiner Zusammensetzung untersucht werden und so ein Copolymerisationsdiagramm erstellt und die Copolymerisationsparameter berechnet werden.

\subsection{Kontrollierte radikalische Polymerisation}

Bei einer konventionellen radikalischen Polymerisation werden Polymerketten unterschiedlicher Länge gebildet, die somit zu einem uneinheitlichen Material führen. Die Molmassenverteilung einer so hergestellten Polymerprobe zeigt deutlich, dass es zwar einen Schwerpunkt gibt, es aber eine Vielzahl an Ketten mit einer deutlich geringeren oder höheren Molmasse gibt. Je nach Anwendung kann dies gewünscht oder auch nachteilig sein. Wenn kompliziertere Strukturen gewünscht sind, die Zusammensetzung gesteuert oder die Molmasse vereinheitlicht werden soll, muss die Polymerisation kontrolliert werden. ${ }^{[25]}$ Für einen idealen Verlauf ist wichtig, dass alle Ketten zur gleichen Zeit initiiert werden, mit derselben Geschwindigkeit wachsen und nicht terminieren. ${ }^{[12]}$ Ein zeitgleicher Kettenstart ist höchstens durch photochemische Initiierung mittels Laserpuls möglich, bei thermischem Initiatorzerfall hingegen werden immer Radikale zeitlich verzögert nachgebildet. Da es sich um einen exponentiellen Zerfall handelt, ist die Konzentration neugebildeter Radikale am Anfang dennoch höher als später. Damit irreversible Terminierungsprozesse unterdrückt werden und ein gleichmäßiges Kettenwachstum möglich ist, müssen die propagierenden Radikale entweder durch reversible Desaktivierung oder reversiblen Kettentransfer vorübergehend der Propagation entzogen werden (siehe Schema 2.6). Ein dynamisches Gleichgewicht stellt ein gleichmäßiges Wachstum aller Ketten und somit ein lineares Anwachsen der Molmasse mit dem Umsatz sicher.

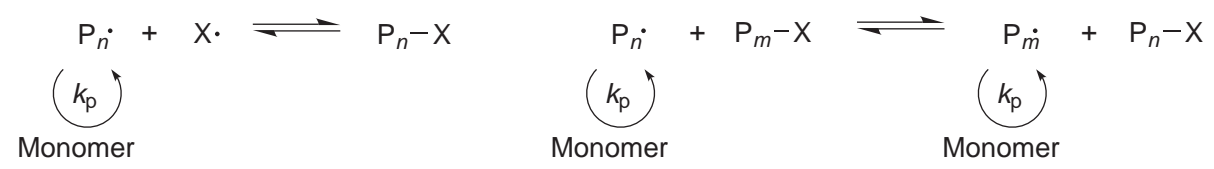

Schema 2.6 Möglichkeiten, eine radikalische Polymerisation zu kontrollieren. Links reversible Desaktivierung, rechts reversibler Kettentransfer, eine Spezialform der reversiblen Desaktivierung. ${ }^{[12]}$ 


\subsubsection{Terminologie}

Polymerisationstechniken, die ein einheitlicheres Polymer liefern, werden in der Literatur unterschiedlich benannt. Es gibt die Ausdrücke kontrollierte Polymerisation, lebend, lebend/kontrolliert, pseudo-lebend, quasi-lebend, lebende Polymerisation mit reversibler Deaktivierung und viele weitere. ${ }^{[26]}$ Zurückzuführen ist dies auf Michael Szwarc, der den Begriff "living polymerization“ im Zusammenhang mit anionischer Polymerisation im Jahr 1956 erstmals aufbrachte. ${ }^{[27]}$ Bei (an)ionischen Polymerisationen können die aktiven Zentren aufgrund ihrer Ladung nicht terminieren. Sie leben und „können wachsen, sobald zusätzliches Monomer hinzugegeben wird“. ${ }^{[27]}$ Des Weiteren finden keine Übertragungsreaktionen statt (Vorraussetzung hierfür ist das Fehlen von Verunreinigungen).

Bei radikalischen Polymerisationen ist es nicht möglich, den Terminus „lebend“ anzuwenden, da Radikale terminieren können (und die Terminierung nie vollständig unterdrückt werden kann, nur bei einigen Polymerisationsarten vernachlässigbar gering ist) und irreversibler Radikaltransfer möglich ist. Obwohl auch hier im Idealfall die Kette weiter wächst, wenn erneut Monomer hinzugegeben wird, kann daher nicht von lebendem Charakter gesprochen werden.

„Kontrolliert" ist ebenfalls ungeeignet, da dieser Begriff in der Polymerchemie eine Vielzahl von Bedeutungen hat. M. Szwarc sagt dazu, dass unterschiedliche Aspekte der Polymerisationen auf unterschiedliche Arten und Weisen kontrolliert werden. Die Temperatur einer exothermen Polymerisation wird z. B. kontrolliert, indem sie in Emulsion durchgeführt wird und die Viskosität von Kautschuk oder Nylon durch Zugabe passender Zusatzstoffe. ${ }^{[28]}$

Von der IUPAC ging im Jahr 2009 die Empfehlung aus, diese Polymerisationen „reversible-deactivation radical polymerization, RDRP“ (reversibel desaktivierende radikalische Polymerisation) zu nennen. ${ }^{[29]}$ Im direkten Zusammenhang mit z. B. den Polymerisationsarten, die in den folgenden Abschnitten vorgestellt werden, darf der Begriff kontrolliert hingegen angewendet werden, da hierbei ersichtlich ist, dass es um eine Kontrolle der Polymerisation durch die jeweiligen Hilfsagenzien geht.

Die drei gängigsten Möglichkeiten für eine „kontrollierte“ radikalische Polymerisation werden im Folgenden in der chronologischen Reihenfolge ihrer Entdeckung vorgestellt. 


\subsubsection{Nitroxidvermittelte Polymerisation, NMP}

NMP steht für „Nitroxide-Mediated Polymerization“ (nach IUPAC [29] auch AMRP, „Aminoxyl-Mediated Radical Polymerization“). Dies war die erste kontrollierte radikalische Polymerisationsart, die im Jahr 1982 von Solomon et al. vorgestellt wurde. ${ }^{[30]}$ Es folgte ein Patent im Jahr $1985^{[31]}$ sowie diverse Übersichtsartikel, ${ }^{[32,33]}$ die den aktuellen Forschungsstand wiedergaben. NMP folgt einem Dissoziations-KombinationsMechanismus (siehe Schema 2.6, links) und macht sich den sogenannten „Persistent Radical Effect“ (PRE) zunutze. Wenn in einer Reaktion sowohl langlebige Radikale (hier: Aminoxyl-Radikale) als auch kurzlebige Radikale (Polymer-Radikale) gebildet werden, da die kurzlebigen z. B. mit einem gleichartigen Radikal terminieren und somit dem Gleichgewicht entzogen werden, baut sich im Laufe der Zeit ein Überschuss an langlebigen Radikalen auf. Die Kreuzreaktion eines kurzlebigen Radikals mit einem langlebigen Radikal wird somit immer wahrscheinlicher. Bei der NMP stellt das Polymerradikal das kurzlebige Radikal dar, welches mit einem anderen Polymerradikal zu terminierten Ketten reagieren kann und somit dem Gleichgewicht entzogen wird (Schema 2.7). Als kontrollierendes Agens, welches mit den Makroradikalen reagiert und diese dem Gleichgewicht entzieht, werden Nitroxide eingesetzt. Das NitroxidRadikal ist äußerst langlebig, es initiiert nicht, terminiert nicht mit einem anderen Nitroxid-Radikal und kombiniert nur mit kohlenstoffzentrierten Radikalen.

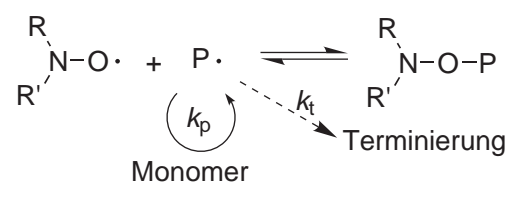

Schema 2.7 Darstellung des NMP-Gleichgewichts. ${ }^{[33]} \mathrm{R}$ und R' sind organische Reste, wobei das Nitroxid auch eine zyklische stickstoffhaltige Verbindung sein kann.

\subsubsection{Atomtransfer-radikalische Polymerisation, ATRP}

ATRP steht für „Atom-Transfer Radical-Polymerization“. Es handelt sich um eine metallkatalysierte Polymerisation, die sich die unterschiedlichen Redoxstufen eines Übergangsmetalls wie z. B. Kupfer zunutze macht und nach dem Atom-ÜbertragungsMechanismus verläuft (siehe Schema 2.6, links). Sie wurde von Matyjaszewski et al. entwickelt und erstmals im Jahr 1995 publiziert. ${ }^{[34,35]}$ Im Jahr 2001 fasste Matyjaszew- 
ski die Entwicklungen der ersten Jahre in einem ausführlichen Übersichtsartikel zusammen. ${ }^{[36]}$

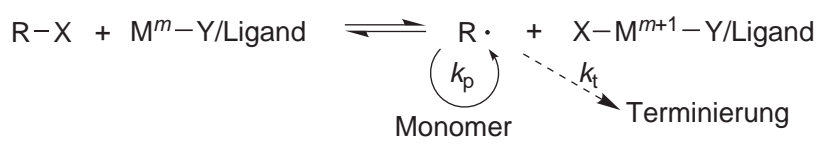

Schema 2.8 Darstellung des ATRP-Gleichgewichts. ${ }^{[36]} \mathrm{M}$ ist ein Übergangsmetall, $\mathrm{Y}$ ist das Gegenion des Komplexes, $\mathrm{X}$ ist ein (Pseudo-)Halogen. Die Oxidationsstufe des Metalls ändert sich bei der Aufnahme von X um +1 .

Das Grundprinzip ist in Schema 2.8 skizziert. Als Initiator dient eine Verbindung, die ein Halogen oder Pseudohalogen trägt. Dieses koordiniert unter Abspaltung eines Radikals an das Metall, welches dadurch oxidiert wird. Das Radikal R. lagert Monomer an und wächst zu einem Makroradikal. Die Radikale können terminieren, wobei diese Terminierung durch das Gleichgewicht unterdrückt wird. Das System wird so gewählt, dass das Gleichgewicht auf der linken Seite bei der desaktivierten Form liegt. In einem ausgewogenen System gibt es nur einen äußerst geringen Anteil an terminierten Ketten und ein gleichmäßiges Wachstum aller Makroradikale, bedingt durch schnelle Initiierung und schnelle reversible Desaktivierung. ${ }^{[36]}$

\subsubsection{Reversibler Additions-Fragmentierungs-Kettentransfer, RAFT}

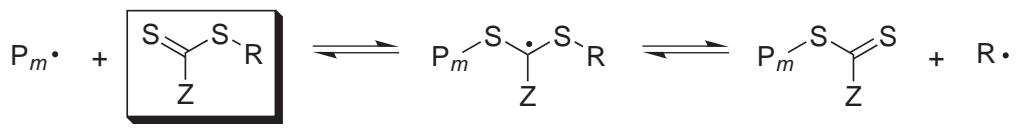

Schema 2.9 Das RAFT-Vorgleichgewicht. ${ }^{[37,38]}$

RAFT steht für "Reversibler Additions-Fragmentierungs-Kettentransfer" und basiert auf dem degenerativen Kettentransfer (siehe Schema 2.6, rechts). Er wurde im Jahr 1998 von Moad, Rizzardo und Thang erstmals publiziert. ${ }^{[37,38]}$ Zeitgleich erschien ein Patent aus Frankreich von Corpart et al., ${ }^{[39]}$ das die kontrollierte Polymerisation von Blockcopolymeren mittels „MADIX“ („Macromolecular Design via the Interchange of Xanthates“) schützt. Wie aus dem Akronym ersichtlich, beschränken sich Copart et al. dabei auf Xanthate ( $Z=$ Alkyloxy). Moad, Rizzardo und Thang verwendeten hingegen anfangs nur Dithioester, die an Alkyl- oder Arylgruppen binden. Der Mechanismus ist jedoch der gleiche, da der Dithioester selber die tragende Rolle spielt. Heute ist die 
Polymerisationsart fast ausschließlich unter dem Namen RAFT verbreitet und umfasst ein weites Spektrum an RAFT-Agenzien, das die Xanthate einschließt. ${ }^{[12,40-43]}$

Die Grundstruktur ist in Schema 2.9 im Kasten dargestellt. Die Z-Gruppe ist die stabilisierende Gruppe, die das Intermediat (Schema 2.9, Mitte) stabilisieren soll und die Addition eines Makroradikals an das RAFT-Agenz beeinflusst. R ist die Abgangsgruppe, die im Vorgleichgewicht abgespalten wird. Somit enthalten manche Polymerketten die Abgangsgruppe R und andere das initiierende Initiatorfragment als Endgruppe. Für eine effiziente RAFT-Polymerisation müssen die folgenden Punkte erfüllt werden: ${ }^{[12]}$

- Das RAFT-Agens enthält eine reaktive Schwefel-Kohlenstoff-Doppelbindung (schnelle Addition des Radikals an das RAFT-Agens).

- Die Intermediat-Radikale fragmentieren (schwache S-R-Bindung), ohne eine Nebenreaktion einzugehen.

- Das Intermediat des Vorgleichgewichts soll bevorzugt die Abgangsgruppe abspalten und nicht das angreifende Radikal.

- Die Radikale R· sollen gut reinitiieren.

Somit ist eine RAFT-Polymerisation eine radikalische Polymerisation, deren Schritte Initiierung, Propagation und Terminierung um das RAFT-Vor- und -Hauptgleichgewicht ergänzt werden (Schema 2.10). Ein reversibler Transferschritt führt zu einem Gleichgewicht zwischen aktiven und inaktiven Ketten und sorgt so für ein gleichmäßiges Wachstum. Terminierung ist im gleichen Maße möglich wie in einer freien radikalischen Polymerisation, außerdem ist die Propagationsgeschwindigtkeit (im Gegensatz zu NMP und ATRP) nicht herabgesetzt. Der „Persistent Radical Effect“ tritt nicht auf.

Das verwendete Monomer beeinflusst die Wahl des RAFT-Agens. Während z. B. Dithioester ( $Z$ = Aryl oder Alkyl) oder Trithiocarbonate $(Z=$ Alkylthio) für Monomere wie Styrol und Methylacrylat geeignet sind, führen sie in einer Vinylacetat-Polymerisation zu Inhibierung oder Retardierung (z. B. durch Kupplung zweier RAFT-Radikale). Umgekehrt sind Carbamate ( $\mathrm{Z}=\mathrm{N}, \mathrm{N}$-Dialkyl oder $\mathrm{N}$-Alkyl-N-Aryl) und Xanthate $(\mathrm{Z}=\mathrm{Al}$ kyloxy), die sich für Vinylacetat eignen, für Styrol und Acrylate ineffizient. ${ }^{[44]}$ Eine Übersicht, die gängige R- und Z-Gruppen und ihre Einsatzgebiete darstellt, ist in Schema 2.11 abgebildet. Für die Z-Gruppen nehmen die Additionsgeschwindigkeiten und Transferkonstanten von links nach rechts ab, während die Fragmentierungsgeschwindigkeit zunimmt. Bei den R-Gruppen nimmt die Fragmentierungsgeschwindig- 


$$
\begin{aligned}
& \text { Initiierung: Startreaktion: } \\
& \ln \stackrel{k_{d}}{\longrightarrow} 2 \mathrm{I} \cdot \quad \mathrm{I} \cdot \mathrm{M} \stackrel{k_{\mathrm{S}}}{\longrightarrow} \mathrm{P}_{1} \text {. }
\end{aligned}
$$

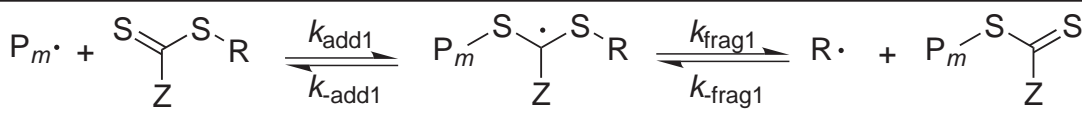

Propagation:

$\mathrm{P}_{m^{*}}+\mathrm{M} \stackrel{k_{\mathrm{p}}}{\longrightarrow} \mathrm{P}_{\mathrm{m}+1} \cdot \quad \mathrm{R} \cdot+\mathrm{M} \stackrel{k_{\mathrm{i}}}{\longrightarrow} \mathrm{P}_{1}$.

Hauptgleichgewicht:

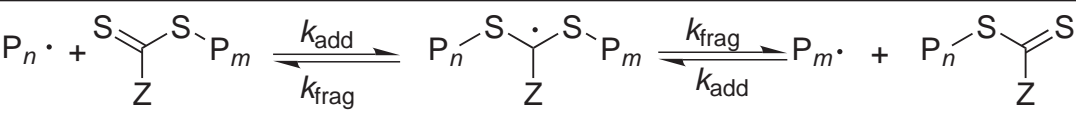

Terminierung:

$$
\mathrm{P}_{m} \cdot+\mathrm{P}_{n} \cdot \stackrel{k_{\mathrm{t}, \mathrm{k}}}{\longrightarrow} \mathrm{P}_{m+n} \quad \mathrm{P}_{m} \cdot+\mathrm{P}_{n} \cdot \stackrel{k_{\mathrm{t}, \mathrm{d}}}{\longrightarrow} \mathrm{P}_{m}+\mathrm{P}_{n}
$$

Schema 2.10 Mechanismus der RAFT-Polymerisation. ${ }^{[40]}$

keit von links nach rechts ab. Eine gestrichelte Linie indiziert eingeschränkte Kontrolle, bedingt durch z. B. Retardierung. ${ }^{\text {[45] }}$

RAFT-Agenzien eignen sich zur Kontrolle fast jeden Monomers, welches sich radikalisch polymerisieren lässt. Neben den in dieser Arbeit verwendeten Vinylmonomeren (Styrol und Vinylacetat), dem Dien 1,3-Butadien und Acrylaten (Bsp. Methylacrylat) können auch Methacrylate, Acrylamide, Methacrylamide und Acrylonitrile verwendet werden. Eine Vielzahl ungeschützter Funktionalitäten an Lösungsmittel- oder Monomermolekülen wird toleriert (z. B. OH, $\left.\mathrm{COOH}, \mathrm{NR}_{2}, \mathrm{C}(\mathrm{O}) \mathrm{NR}_{2}, \mathrm{SO}_{3} \mathrm{H}\right) .{ }^{[12]}$ Die Reaktionsbedingungen können ebenfalls flexibel an den Bedarf angepasst werden. ${ }^{[46]}$ So ist es möglich, die Polymerisation in Substanz, in wässriger oder organischer Lösung oder in Emulsion durchzuführen. 


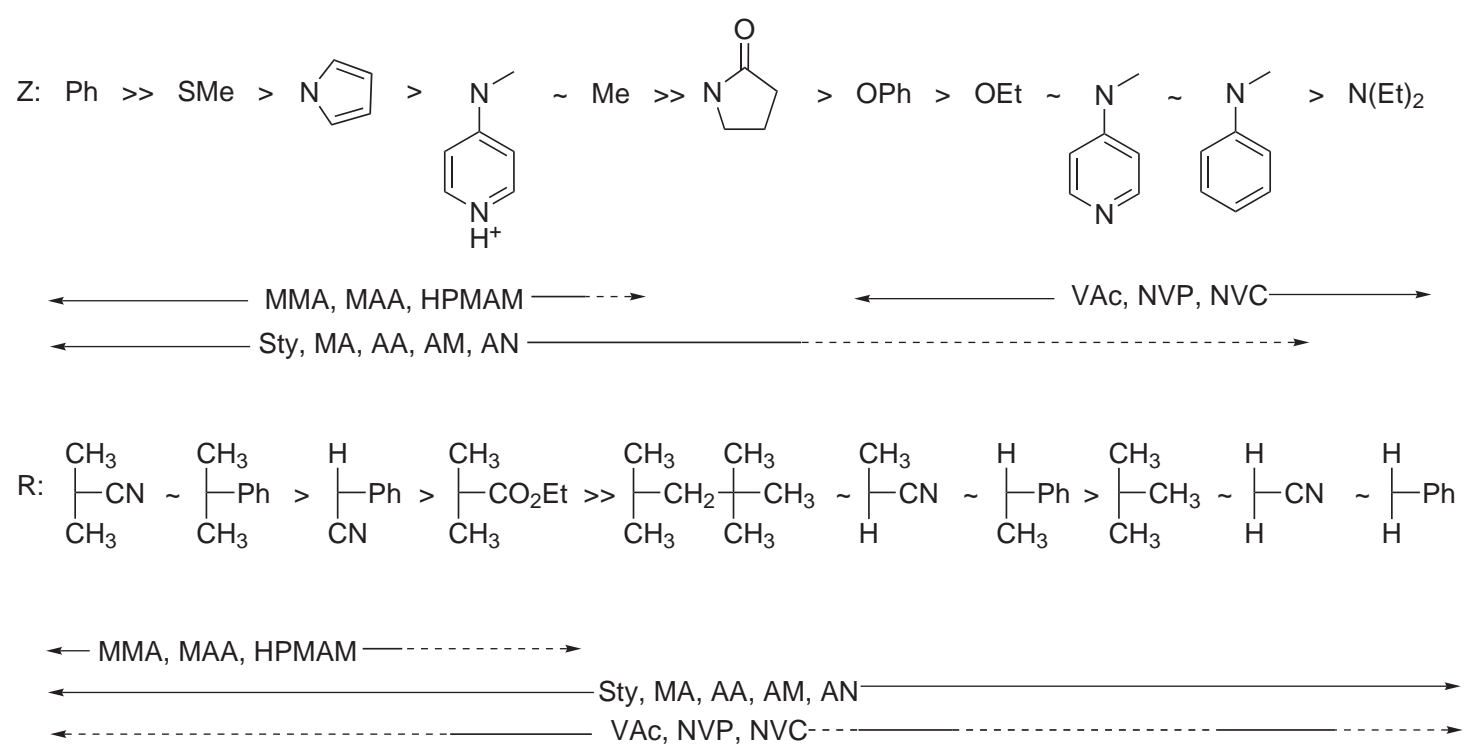

Schema 2.11 Schematische Darstellung potenzieller R- und Z-Gruppen und ihrer Einsatzmöglichkeiten. ${ }^{[45]}$

\section{Schaltbare RAFT-Agenzien}

RAFT-Agenzien eignen sich hervorragend für die Synthese von Blockcopolymeren. Das Makro-RAFT-Agens, welches bereits einen Block von Monomer 1 trägt, kann erneut mit Initiator und einem neuen Monomer versetzt werden. Der Block des Monomers 2 wird zwischen die RAFT-Einheit und Block 1 eingebaut. Dies funktioniert aber nur dann, wenn die Monomere und die entsprechenden Radikale ähnliche elektronische Eigenschaften haben, das Intermediat unter Abspaltung des ersten Blockes fragmentiert und das abgespaltene Makroradikal in einer Polymerisation von Monomer 2 gut reinitiiert. Styrol und Butadien zählen ebenso wie die Acrylate zu der Klasse der sogenannten „more activated monomers" (MAM), wohingegen z. B. Vinylacetat zu den „less activated monomers" (LAM) gerechnet wird. RAFT Agenzien, die die Polymerisation von MAMs effektiv kontrollieren (wie z. B. Dithioester oder Trithiocarbonate), inhibieren oder retardieren die Polymerisation von LAMs. Umgekehrt sind Dithiocarbamate und Xanthate, die sich in der Polymerisation von LAMs bewährt haben, für eine MAMPolymerisation ungeeignet. ${ }^{[4]}$

Verschiedene Forschungsgruppen ${ }^{[47,48]}$ haben unterschiedliche kontrollierte Polymerisationen kombiniert, um ein PolyMAM-block-polyLAM zu synthetisieren. Dabei wird z. B. der erste Block mittels ATRP hergestellt, anschließend wird die ATRP-InitiatorFunktionalität (ein endständiges Bromatom) in eine Xanthat-Endgruppe umgewan- 
delt und der zweite Block (in diesem Fall der Vinylacetat-Block) in einer RAFTPolymerisation synthetisiert.

Sollen beide Blöcke mittels RAFT hergestellt werden, um z. B. sicherzustellen, dass das Polymer restlos metallfrei ist, gibt es die Möglichkeit sogenannte "switchable RAFT agents", ${ }^{44-51]}$ also schaltbare RAFT-Agenzien, zu benutzen. Diese RAFT-Agenzien bieten die Möglichkeit, durch Protonierung bzw. Deprotonierung die elektronischen Eigenschaften so zu verändern, dass entweder LAMs oder MAMs kontrolliert werden können.

In Schema 2.12 ist das Grundprinzip dargestellt. Die von Benaglia et al. vorgestellten RAFT-Agenzien gehören zu den Dithiocarbamaten. In die Z-Gruppe ist ein Pyridinrest integriert, der am Stickstoff protoniert werden kann und somit die elektronische Struktur und die Eigenschaften des RAFT-Agenzes ändert.

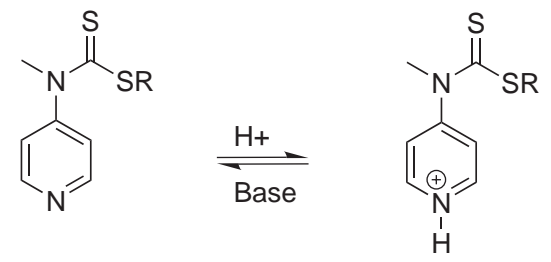

Schema 2.12 Schematische Darstellung eines schaltbaren RAFT-Agenzes. ${ }^{[49,50]}$

Schema 2.13 zeigt die Verwendung am Beispiel eines RAFT-Agenzes mit einer Methylacrylat-Abgangsgruppe. Aus dem neutralen RAFT-Agenz wird durch Protonierung das geladene Pendant gebildet, durch Deprotonierung mittels einer Base (z. B. Filtration durch Natriumcarbonat oder stöchiometrische Zugabe 4-(Dimethylamino)pyridin (DMAP)) wird wieder das neutrale RAFT-Agens oder Makro-RAFT-Agens gewonnen. Eine mögliche grafisch-darstellbare Grenzstruktur für das neutrale bzw. geladene RAFT-Agens ist ebenfalls gezeigt. In einer RAFT-Polymerisation von LAMs wie Vinylacetat findet das ungeladene RAFT-Agens Verwendung, in einer RAFTPolymerisation von MAMs wie Styrol wird hingegen die geladene Spezies verwendet. In der Praxis hat es sich bewährt, zuerst den MAM-Block zu polymerisieren, da terminale LAM-Gruppen schlechtere Radikalabgangsgruppen als terminale MAM-Gruppen sind (siehe Schema 2.14). ${ }^{[49]}$ 

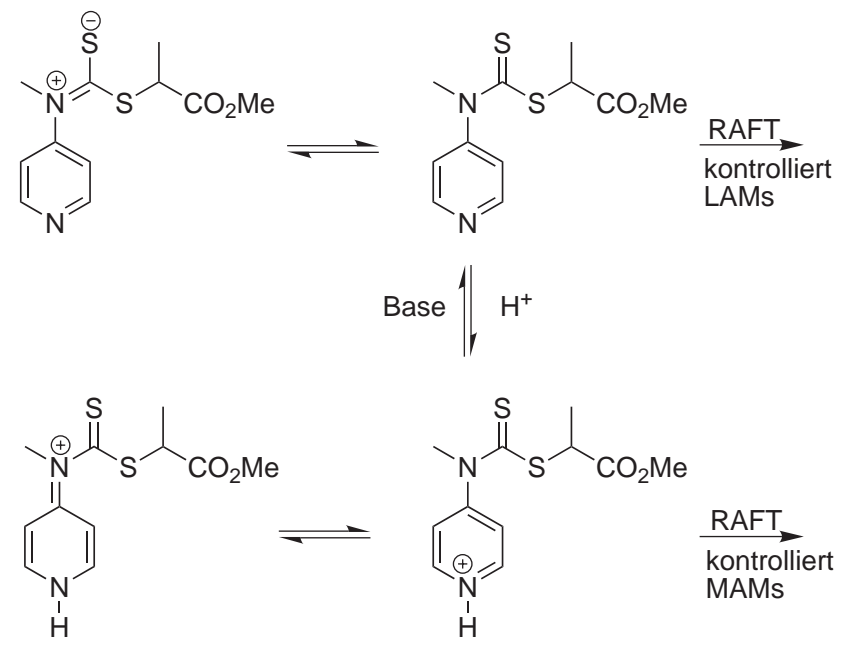

Schema 2.13 Darstellung der Resonanzstrukturen der schaltbaren RAFT-Agenzien am Beispiel von Methyl-2-(methyl(pyridin-4-yl)carbamothioyl)thiopropanoat. ${ }^{[49]}$<smiles>CN(C(=S)SF)c1cc[nH+]cc1</smiles><smiles>CSC(=S)N(C)c1ccncc1</smiles>

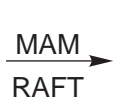

$\overrightarrow{\text { RAFT }}$ $\underset{\mathrm{RAFT}}{\stackrel{\mathrm{LAM}}{\longrightarrow}}$

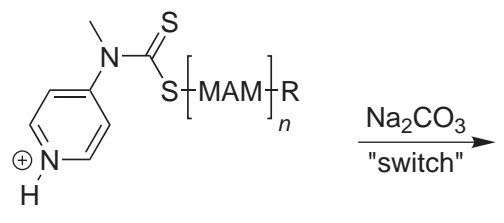<smiles>CN(C(=S)SCC(C)(C)C)c1ccncc1</smiles>

Schema 2.14 Synthese eines PolyMAM-block-polyLAM. Das Umschalten (englisch: „switch“) des RAFT-Agens erfolgt mit Natriumcarbonat. 


\section{Molmassenberechnungen bei RAFT-Polymerisationen}

Es gibt unterschiedliche Möglichkeiten, das zu erwartende Molmassen-Zahlenmittel einer RAFT-Polymerisation zu berechnen.

Ein einfacher, allgemeiner Ansatz wurde im Jahr 2005 von Moad et al. vorgestellt: ${ }^{[12]}$

$$
\bar{M}_{\mathrm{n}}^{\text {theo }}=\frac{[\mathrm{M}]_{0}-[\mathrm{M}]_{t}}{[\mathrm{RAFT}]_{0}} \cdot M_{\mathrm{M}}=\frac{U \cdot[\mathrm{M}]_{0}}{[\mathrm{RAFT}]_{0}} \cdot M_{\mathrm{M}}
$$

Hierbei wird das verbrauchte Monomer, also die Differenz zwischen der Ausgangskonzentration $[\mathrm{M}]_{0}$ und der Konzentration zur Zeit $t,[\mathrm{M}]_{t}$, mit der Molmasse des Monomers $M_{\mathrm{M}}$ multipliziert und durch die Anzahl der Ketten, welche der RAFT-AgensAusgangskonzentration [RAFT $]_{0}$ entspricht, dividiert. Um sinnvolle Ergebnisse zu erhalten, muss zu der Molmasse, die über o.g. Formeln berechnet werden kann, die Molmasse des RAFT-Agenzes $M_{\text {RAFT }}$ hinzuaddiert werden, da jede Polymerkette ein RAFT-Molekül enthält und bei einem Umsatz null die Molmasse des Produkts der Molmasse des RAFT-Agens entspricht.

Es ergibt sich für die theoretische Molmasse ohne Berücksichtigung der Initiatorkonzentration: ${ }^{[12]}$

$$
\bar{M}_{\mathrm{n}}^{\text {theo }}=\frac{U \cdot[\mathrm{M}]_{0}}{[\mathrm{RAFT}]_{0}} \cdot M_{\mathrm{M}}+M_{\mathrm{RAFT}}
$$

Zeitgleich zu Moad et al. ${ }^{[12]}$ veröffentlichten Arita et al. ${ }^{[52]}$ und Monteiro ${ }^{[53]}$ eine etwas ausführlichere Variante, in der die Terminierung und die dadurch entstehenden Polymerketten berücksichtigt werden:

$$
\bar{M}_{\mathrm{n}}^{\text {theo }}(\mathrm{T})=\frac{[\mathrm{M}]_{0}-[\mathrm{M}]_{t}}{[\mathrm{RAFT}]_{0}+d \cdot f \cdot\left([\mathrm{I}]_{0}-[\mathrm{I}]_{t}\right)} \cdot M_{\mathrm{M}}+M_{\mathrm{RAFT}}
$$

Wenn die Geschwindigkeitskoeffizient $k_{\mathrm{d}}$ bekannt ist, ${ }^{[54]}$ kann die Molmasse mit folgender Formel berechnet werden:

$$
\bar{M}_{\mathrm{n}}^{\text {theo }}(\mathrm{T})=\frac{U \cdot[\mathrm{M}]_{0}}{[\mathrm{RAFT}]_{0}+[\mathrm{I}]_{0} \cdot f \cdot d \cdot\left(1-\exp \left(-k_{\mathrm{d}} \cdot t\right)\right)} \cdot M_{\mathrm{M}}+M_{\mathrm{RAFT}}
$$

Neben dem verbrauchten Monomer und RAFT-Agens spielt der bis zum Zeitpunkt $t$ zerfallene Initiator und die Polymerketten, die daraus gebildet wurden, eine Rolle. Die Inititatoreffizienz $f$ und $d$ als Maß für den Terminierungsmechanismus werden ebenfalls berücksichtigt $\left(f=0,6\right.$ für AIBN als Initiator bei $60^{\circ} C^{;}{ }^{[55]} d=1,0$ für Styrol ${ }^{[55]}$ und $d=1,67^{[12]}$ bzw. $1,70^{[56]}$ für MMA).

Diese Erweiterung beinhaltet Polymerketten, die keine RAFT-Gruppe tragen und nur 
durch Addition von Monomer an ein Inititatorradikal entstanden sind. Der Initiatorzerfall läuft zeitlich verzögert ab und liefert permanent neue Radikale nach, denen die Terminierung mittels Kombination oder Disproportionierung gegenübersteht. Es gibt daher Polymerketten, die keine RAFT-Gruppe tragen, die aber trotzdem beim Umsatz berücksichtigt werden müssen. Für die Auswertung der Polymerisationen wurde Formel 2.40 verwendet.

\subsection{Elastomere}

Der Duden ${ }^{[57]}$ definiert ein Elastomer als „synthetischer Kautschuk oder gummiähnlicher Kunststoff“, wobei Gummi ein „durch Vulkanisation aus natürlichem oder synthetischem Kautschuk hergestelltes Produkt von hoher Elastizität" ist. Für die Einteilung von Polymeren gibt es verschiedene Möglichkeiten. Eine davon klassifiziert sie nach ihrem mechanischen Verhalten, das z. B. in einem Zugversuch für den eindimensionalen Fall untersucht werden kann. An einen Probekörper mit definierten Maßen wird eine Zugspannung $\sigma$ angelegt und dessen Dehnung $\varepsilon$ gemessen. In einem SpannungsDehnungs-Diagramm lassen sich die Ergebnisse grafisch darstellen. Aus der Steigung im lineare Anfangsbereich lässt sich der E-Modul (auch: Young-Modul) des Polymers nach dem Hooke'schen Gesetz (Formel 2.41) berechnen.

$$
\sigma=E \cdot \varepsilon
$$

Bei stärkerer Dehnung weicht das Verhalten deutlich von der Linearität ab. Die maximal mögliche Dehnung liegt für Elastomere bei 500 - $1000 \%$. ${ }^{[58]}$

Entropieelastische Polymere (Elastomere) haben einen deutlich geringeren E-Modul als energieelastische Polymere. $\mathrm{Zu}$ den Elastomeren zählen neben Naturkautschuk (Poly(isopren)) auch Poly(butadien), Poly(isobuten), Poly(chloropren), Poly(dimethylsiloxan) („,Silikon“) sowie diverse Copolymere. ${ }^{[9,59,60]}$ Wichtig ist eine lange Polymerkette mit möglichst wenig Wechselwirkungen zu benachbarten Ketten (keine Wasserstoffbrückenbindungen, sterischen Hinderungen o. ä.). Bei der Belastung haben diese Materialien mehr Spielraum, da lange Moleküle gleichbedeutend sind mit mehr Konformationszuständen, die das Polymer bei Zugbelastung einnehmen kann. Eine Quervernetzung zwingt das Polymer nach Ende der Belastung in seinen Ausgangszustand zurück. Diese Quervernetzungen sind teilweise bereits im Polymer enthalten oder können zusätzlich noch hinzugefügt werden (siehe Abschnitt 2.6).

Darüber hinaus gibt es thermoelastische Effekte. Gough machte im Jahr 1805 die Beob- 
achtung, dass sich ausgedehnter Gummi unter konstanter Belastung bei Erwärmung zusammenzieht und umgekehrt reversibel Hitze abgibt, wenn er gedehnt wird. Diese Beobachtungen wurden im Jahr 1859 von Joule bestätigt, der mit weiter entwickelten Materialien arbeiten konnte. Diese beiden Effekte sind als Gough-Joule-Effekte bekannt. ${ }^{[58]}$ Metalle verhalten sich komplett gegensätzlich; ein Probekörper aus Stahl kühlt sich bei Dehnung aufgrund von Zugbelastung ab. Bei Elastomeren hat die Aufheizung entropische Gründe. Wenn an das Polymer eine Zugspannung angelegt wird, werden die langen Polymerketten gedehnt und in eine Vorzugsrichtung gezwungen. Dabei kommt es zu Wechselwirkungen zwischen den Molekülen, die Probe erwärmt sich. Bei Metallen wie Stahl werden die Atome aus ihrer Gleichgewichtsposition heraus verschoben. Die Energie dafür muss das System zur Verfügung stellen, die Probe kühlt sich ab. ${ }^{[61]}$

Im Gegensatz zu Duroplasten sind Elastomere nur schwach (weitmaschig) vernetzt, haben eine geringe Zugfestigkeit, eine geringe Steife und eine „mittlere bis große Reißdehnung verbunden mit großer Rückfederung“. ${ }^{[62]}$ Die Reißdehnung, auch Bruchdehnung genannt, beschreibt die Fähigkeit eines Werkstoffes, sich ohne Riss verformen zu lassen. Sie ist definiert als das Verhältnis der Längenänderung bis zum Riss zur Ausgangslänge und wird in Prozent angegeben. Elastomere sind in Bereichen von unter $0^{\circ} \mathrm{C}$ bis zur Zersetzungstemperatur gummielastisch und eignen sich somit für eine Vielzahl von Anwendungen (s.o.).

\subsection{Vulkanisation}

Kautschuk wird erst durch die Vulkanisation, eine Vernetzungsreaktion, zu einem wertvollen Werkstoff. Es entstehen unter Druck bei hohen Temperaturen (i.d. R. über $140^{\circ} \mathrm{C}$ ) neue chemische Bindungen, die aus den einzelnen Polymerketten ein Netzwerk bilden. Ein Fließen des Polymers ist nach erfolgter Vernetzung kaum noch möglich. Die sog. Vernetzungsdichte hat einen Einfluss auf eine Vielzahl an mechanischen Eigenschaften, wie z. B. die Zugfestigkeit, elastische Rückstellkräfte und den Reibungskoeffizienten. ${ }^{[4]}$ Häufig werden die Begriffe „Vernetzung“ und „Vulkanisation“ synonym verwendet. Streng genommen beschreibt "Vernetzung“ die chemische Reaktion und „Vulkanisation“ den technischen Prozess. ${ }^{[8]}$ Bei der Vulkanisation gibt es unterschiedliche Kenngrößen. Eine optimale Vernetzungsdichte entspricht einem Vernetzungsgrad von $100 \%$. Bereits bei einem Vernetzungsgrad von lediglich $10 \%$ ist eine plastische Verformung nicht mehr möglich. Spätestens zu diesem Zeitpunkt muss die Vulkanisa- 
tionsmischung ihre endgültige Form eingenommen haben, da sie sich jetzt nicht mehr verformen lässt. Dies ist die sog. Anvulkanisationszeit. Die Ausvulkanisationszeit ist erreicht, wenn der Vernetzungsgrad $90 \%$ beträgt. Zu diesem Zeitpunkt kann die äußere Wärmezufuhr abgeschaltet werden, da die enthaltene Restwärme bedingt durch die schlechte Wärmeleitfähigkeit von Kautschuk zu einer vollständigen Ausvulkanisation führt. ${ }^{[8]}$ Gemessen wird der Vulkanisationsgrad über das Drehmoment, welches zur Verformung einer Probe bei der Vulkanisationstemperatur notwendig ist.

Nach erfolgter Vernetzung hat das Polymer eine deutlich höhere benötigte Zugkraft, die mit einer höheren Rückstellkraft einhergeht (Abbildung 2.1). Im Vergleich zu unvernetztem Kautschuk ist also eine höhere Kraft für die gleiche Dehnung erforderlich, die von einem Zusammenziehen auf die Ausgangslänge (Elastomer) bzw. geringem Zusammenziehen (Kautschuk) gefolgt ist.

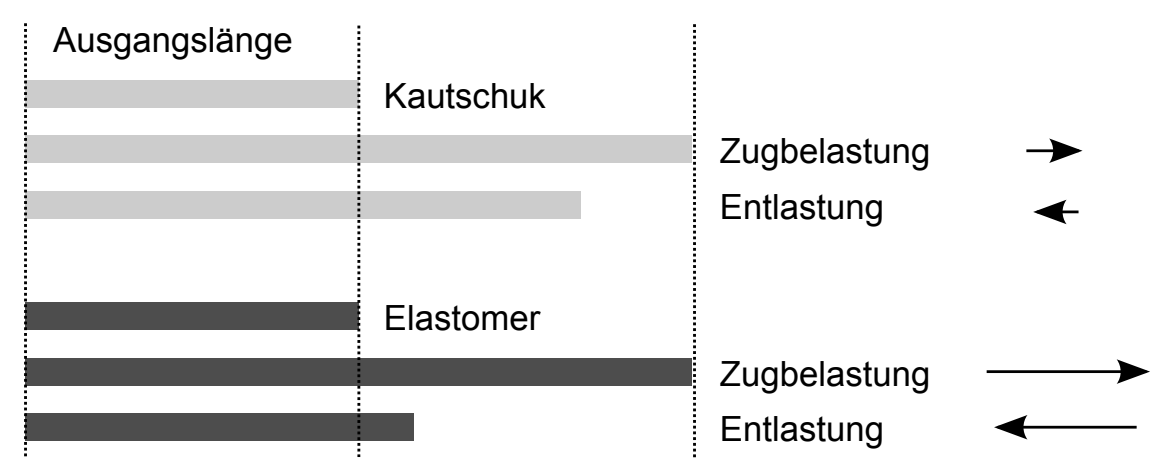

Abbildung 2.1 Schematische Darstellung des Verhaltens von Kautschuk und Elastomeren bei Be- und Entlasung sowie der dazu benötigten Kraft nach Abts. ${ }^{[8]}$

\subsubsection{Additive A4,8] $^{[4}$}

Es wird zwischen der Schwefelvernetzung (für NR, SBR, BR, NBR und andere Dienkautschuke) und der Peroxidvernetzung (für gesättigte Polymere) unterschieden, ${ }^{[4]}$ wobei die Schwefelvernetzung deutlich älter ist. Bereits im frühen 19. Jahrhundert gelang es Charles Goodyear ein Gemisch aus Kautschuk und elementarem Schwefel mit der Hitze eines heißen Ofens zur Vernetzung anzuregen. Es bilden sich Sulfidbrücken, die zwei Polymerketten miteinander vernetzen. Bei der Peroxidvernetzung zerfällt ein (meist symmetrisches) Peroxid thermisch. Durch eine Übertragungsreaktion eines Peroxidradikals auf eine Polymerkette entsteht ein Makroradikal, welches mit einem weiteren Makroradikal reagiert und eine neue chemische Bindung ausbildet. 
Heutzutage werden neben Schwefel noch weitere Additive hinzugegeben. Elementarer Schwefel oder andere Schwefelspender (thermisch labile Organo-SchwefelVerbindungen) bilden die Vernetzungsstellen. Da Schwefel als $\mathrm{S}_{8}$-Molekül vorliegt, sind die Polymerketten mit Ketten aus bis zu 8 Schwefelatomen verbrückt. Dies führt zu einer erhöhten Beweglichkeit im Vergleich zu Schwefelspendern, die kürzere Brücken bilden. Beschleuniger erhöhen die Vulkanisationsgeschwindigkeit und Aktivatoren beeinflussen die Wirksamkeit des Beschleunigers positiv. Fettsäuren wie Stearinsäure erhöhen die Löslichkeit des Vernetzers im Kautschuk, da sie lösliche Komplexe bilden. Eine Erhöhung des pH-Wertes durch Zugabe basischer Metalloxide aktiviert die Vernetzung ebenfalls. Vulkanisationsverzögerer helfen, kurze Vulkanisationszeiten besser steuern zu können, indem die Fließperiode am Anfang der Vulkanisation herausgezögert wird und z. B. die Vulkanisationsmischung sich gut in der Form verteilen kann.

\subsection{Auswahl der Monomere}

Obwohl Naturkautschuk aus dem Monomer Isopren aufgebaut ist, wird bei Synthesekautschuk meistens auf 1,3-Butadien zurückgegriffen. Neben 1,3-Butadien gibt es noch das Isomer 1,2-Butadien, welches hier keine Verwendung fand. Im Folgenden wird daher „Butadien“ als Kurzform für 1,3-Butadien verwendet. Darüber hinaus ist es möglich und üblich, Copolymerisate aus Butadien und einem oder mehreren weiteren Monomeren zu erstellen, wie z. B. Styrol-Butadien-Kautschuk (SBR, engl. „styrene butadiene rubber"). Während Polystyrol zu den thermoplastischen Polymeren gehört, die in einem bestimmten Temperaturbereich verformbar sind, handelt es sich bei StyrolButadien-Kautschuk um ein Elastomer.

Butadien benötigt als Gas einen speziellen apparativen Aufbau, wohingegen das flüssige Monomer Styrol leichter in der Handhabung ist. Es ist z. B. möglich, Ansätze unterschiedlicher Konzentrationen zeitgleich zu polymerisieren, was bei Butadien nicht möglich ist, da nur ein Hochdruckautoklave geeigneter Größe zur Verfügung steht. Weiterhin können andere unpolare Monomere von Interesse sein, wie z. B. Ethylen, da dies eine höhere Propagationsgeschwindigkeit aufweist. Ethylen ist wie Butadien ein gasförmiges Monomer, das normalerweise bei hohen Drücken polymerisiert wird. Als Modellsystem für Ethylen bietet sich Vinylacetat an.

Die Eignung von Styrol als Modellsystem für Butadien und von Vinylacetat für Ethylen beruht auf der Ähnlichkeit der Monomere in ihrem Polymerisationsverhalten. 


\subsubsection{Copolymerisationsparameter}

Die Ähnlichkeit von Monomeren in ihrem Polymerisationsverhalten kann z. B. mit Hilfe der Copolymerisationsparameter begründet werden. Wie in Kapitel 2.3 bereits erläutert, sind die Copolymerisationsparameter definiert als das Verhältnis der Geschwindigkeitskoeffizienten für die Anlagerung des Monomers, welches mit der radikaltragenden Gruppe identisch ist, im Vergleich zu der Anlagerung des anderen Monomers. Sind die Copolymerisationsparameter beide (nahe) 1, ist die Addition beider Monomerspezies gleich wahrscheinlich. Es bildet sich ein statistisches Copolymer, dessen Zusammensetzung diejenige der Monomermischung wiederspiegelt (ideale azeotrope Copolymerisation). Aus Tabelle 2.1 geht hervor, dass Butadien und Styrol sowie Butadien und para-Chlorstyrol (als Beispiel für ein para-substituiertes Styrolderivat mit elektronenziehendem Substituenten) Copolymerisationsparameter nahe 1 haben und somit ein ähnliches Polymerisationsverhalten aufweisen.

Das System Ethylen/Vinylacetat hat je nach Druck und Temperatur Copolymerisationsparameter von fast genau 1 oder knapp über 1. Obwohl diese beiden Monomere sich in ihrer Molekülstruktur deutlich unterscheiden, stellen sie damit ein perfektes Copolymerisationssystem für statistische Polymerisationen dar. Grund dafür ist die geringe Stabilisierung der radikalischen Funktionalität durch die Acetoxy-Gruppe beim Vinylacetat. Einzig die unterschiedlichen Aggregatzustände machen die direkte Übertragung der Polymerisationsbedingungen schwierig.

Eine Copolymerisation von Styrol mit Vinylacetat zeigt ein starkes nichtideales (nichtazeotropes) Verhalten. Aufgrund der hohen thermodynamischen Stabilität von StyrolRadikalen addieren Styrol-Moleküle besonders in Gegenwart von Vinylacetat, welches hoch instabile Radikale bildet, bevorzugt. Die Folge ist eine starke Tendenz zur Blockbildung.

Tabelle 2.1 Copolymerisationsparameter für unterschiedliche Monomere. ${ }^{[63-66]}$

\begin{tabular}{llccl}
\hline Monomer 1 & Monomer 2 & $r_{1}$ & $r_{2}$ & Bedingungen \\
\hline Butadien & Styrol & 1,39 & 0,78 & $\mathrm{~T}=60^{\circ} \mathrm{C}$ \\
Butadien & para-Chlorstyrol & 1,220 & 1,600 & $\mathrm{~T}=50^{\circ} \mathrm{C}$ \\
Ethylen & Vinylacetat & 1,2 & 1,1 & $\mathrm{~T}=220-240{ }^{\circ} \mathrm{C}, p=2000 \mathrm{bar}$ \\
Ethylen & Vinylacetat & 1,01 & 1,0 & $\mathrm{~T}=150^{\circ} \mathrm{C}, p=850 \mathrm{~atm}$ \\
Styrol & Vinylacetat & 55 & 0,01 & $\mathrm{~T}=60^{\circ} \mathrm{C}$ \\
\hline
\end{tabular}


Abbildung 2.2 zeigt die Copolymerisationsdiagramme für die Copolymerisation von Butadien mit Styrol (oben links) und para-Chlorstyrol (oben rechts), für Ethylen mit Vinylacetat (unten links) und für Styrol mit Vinylacetat (unten rechts). Aufgetragen wurde die Konzentration im Polymer gegen die Konzentration in der Monomermischung. Für eine beliebige Ausgangskonzentration [X] kann der Anteil der beiden Monomere im Polymer $[\mathrm{X}(\mathrm{P})]$ abgelesen werden. Die schwarze Linie stellt das Idealverhalten dar $\left(r_{1}=r_{2}\right)$.
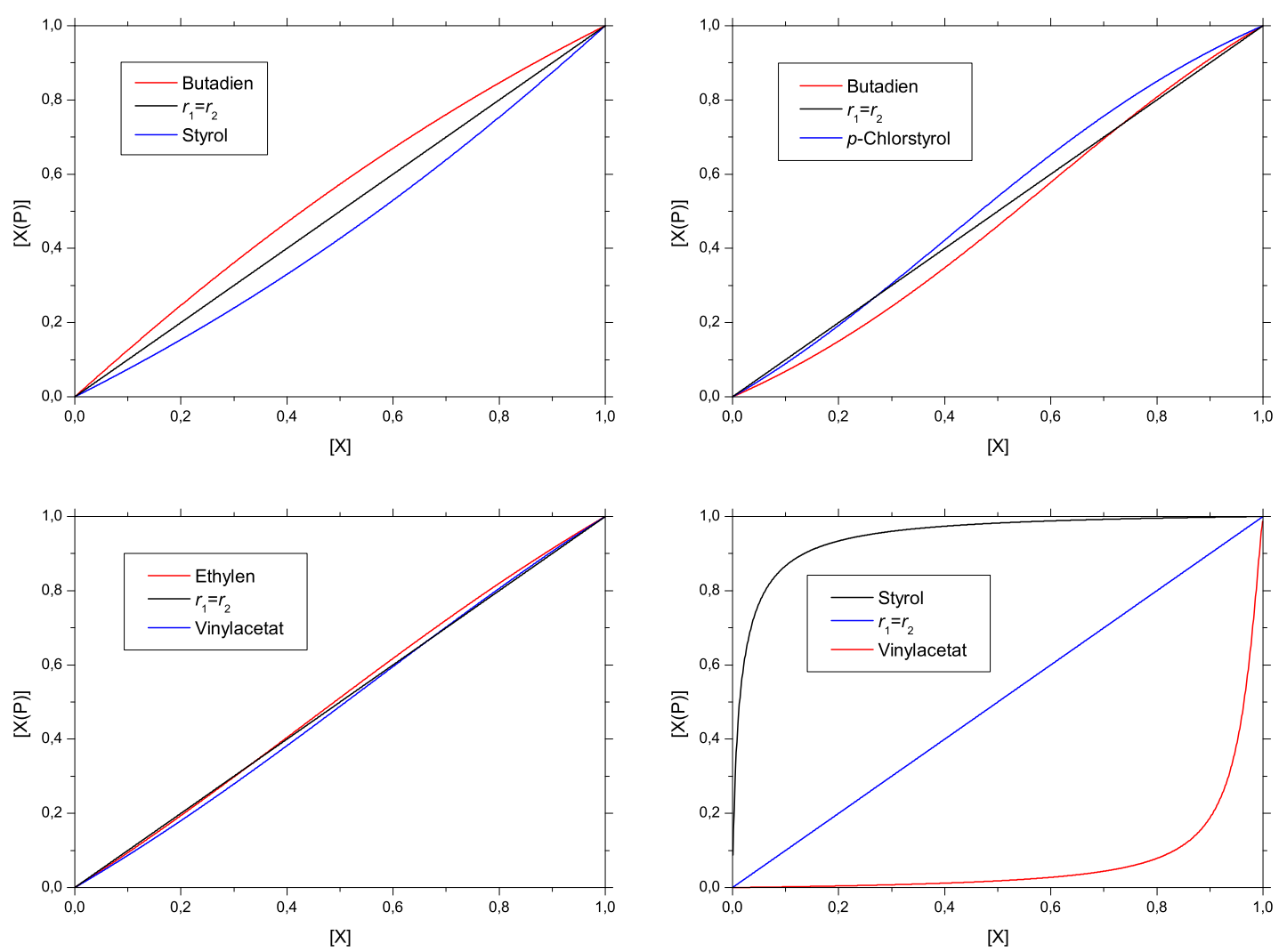

Abbildung 2.2 Copolymerisationsdiagramme für Butadien und Styrol (oben links), ${ }^{[64]}$ Butadien und $p$-Chlorstyrol (oben rechts), ${ }^{[65]}$ Ethylen und Vinylacetat (unten links) ${ }^{[63]}$ und Styrol und Vinylacetat (unten rechts). ${ }^{[64]}[\mathrm{X}]=$ Konzentration von $\mathrm{X}$ in der Monomermischung, $[\mathrm{X}(\mathrm{P})]=$ Konzentration von $\mathrm{X}$ im Polymer. 


\section{3 \\ Zielsetzung}

Ziel dieser Arbeit ist die Herstellung von funktionalisierten Synthesekautschuken für technische Anwendungen mittels kontrollierter radikalischer Polymerisation. Hierfür bietet sich die RAFT-Polymerisation an, da sie sehr variabel hinsichtlich der Polymerisationsbedingungen ist und die $\mathrm{zu}$ verwendenden Monomere sich damit gut kontrollieren lassen. Synthesekautschuk besteht aus Polybutadien oder Butadien-StyrolCopolymeren, seltener Polyisopren.

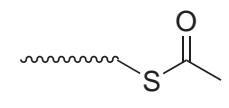

1

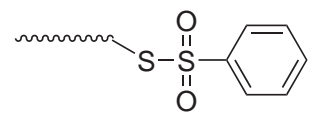

2

Schema 3.1 Abbildung der potenziell vulkanisierbaren Gruppen, die verwendet wurden.

Die beiden funktionellen Gruppen, die eingebaut werden sollen, sind in Schema 3.1 dargestellt. Sowohl beim S-Thioacetat 1 als auch beim Benzolthiosulfonat $\mathbf{2}$ erfolgt dies über ein verbrückendes Schwefelatom (dem Namen von Thioestern wird in Kursivdruck das Atom, welches verbrückend wirkt, voran gestellt. Da bei Thioestern eine Verbrückung über den Schwefel die Regel ist und eine Umlagerung erst bei hohen Tem- 
peraturen auftritt und somit ausgeschlossen werden kann, wird im Folgenden auf den Zusatz "S" verzichtet).

Durch die gezielt eingebauten funktionellen Gruppen können Knotenpunkte während der Vulkanisation entstehen. Während zu einer konventionellen Vulkanisation Schwefel, Beschleuniger, Vernetzer und andere Chemikalien zugegeben werden, sollen diese bei dem Einsatz eines funktionalisierten Polymers weitestgehend überflüssig sein. Des weiteren kann durch eine $\alpha, \omega$-Funktionalisierung eines RAFT-Agens ein Einbinden der Kettenenden gewährleistet werden, welche sonst frei im Polymer verbleiben.

Es gibt unterschiedliche Möglichkeiten, die beiden Funktionalitäten an ein Monomer oder RAFT-Agens anzubinden. Generell ist die Funktionalisierung des Ausgangsmaterials der Funktionalisierung des fertigen Polymers vorzuziehen, da sie selektiver erfolgt und eventuelle Lösungsprobleme umgangen werden.

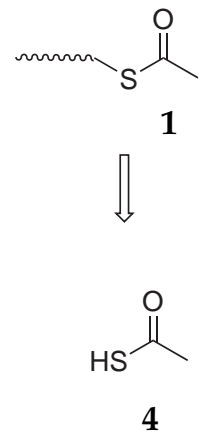

4
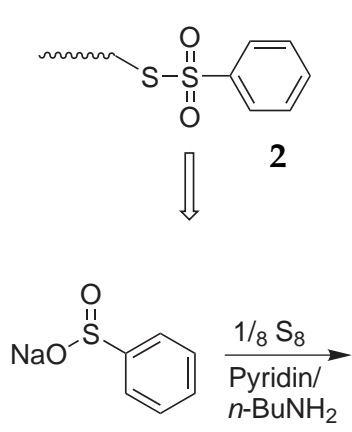

6

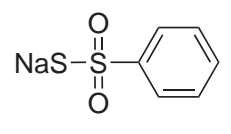

5
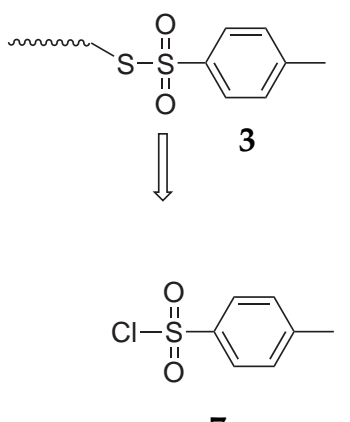

7

Schema 3.2 Zugang zu den funktionellen Gruppen.

Ein Thioacetatester 1 kann durch Veresterung eines Thiols ${ }^{[67]}$ mit Essigsäure gebildet werden, in einer nukleophilen Substitution mit einer halogenorganischen Verbindung ${ }^{[68]}$ oder durch Addition von Thioessigsäure 4 an eine Doppelbindung. ${ }^{[69]}$ Neben Thioessigsäure 4 kann auch Kaliumthioacetat verwendet werden. ${ }^{\text {[0,71] }}$

Für Funktionalität 2 ist ein Syntheseschritt erforderlich, um das Ausgangsmaterial zu erhalten. Aus dem kommerziell erhältlichen Natriumbenzolsulfinat $\mathbf{6}$ wird mit elementarem Schwefel und einer starken Base quantitativ das gewünschte Produkt, Natriumbenzolthiosulfonat 5, gebildet. Diese Synthese ist literaturbekannt. ${ }^{[72]}$ Das Salz kann in geeigneten Lösungsmitteln gelöst und zur Reaktion gebracht werden. Alternativ kann es mit einem Disulfid und Iod zu zwei Äquivalenten Benzolthiosulfonatverbindung reagieren. ${ }^{[73]}$

Die genaue Struktur des organischen Restes von 2 ist nebensächlich; eine Methylgruppe in para-Position, die z. B. durch Verwendung von para-Toluolsulfonylchlorid 7 einge- 
führt werden würde (Schema 3.2, Verbindung 3), könnte toleriert werden. Dieser Weg wurde jedoch nicht weiter verfolgt, da die Anbindung an Thiole erfolgen müsste, die nur in begrenztem Maße kommerziell erhältlich sind, schwierig in der Synthese und aufgrund ihres charakteristischen Geruchs unangenehm in der Anwendung sind.

Eine Kombination von funktionalisierten RAFT-Agenzien und Monomeren führt zu Polymeren, die mehrere Netzknotenpunkte entlang der Kette enthalten und inklusive der beiden Enden in das durch Vulkanisation erzeugte Polymernetzwerk eingegliedert sind. Im Rahmen dieser Arbeit wurden erste Experimente in der Regel mit Styrol durchgeführt (siehe 2.7). Erfolgversprechende Polymerisationen wurden mit Butadien wiederholt.

Die Aufgabenstellung stammt von der Abteilung „Advanced Tire Materials“ der Continental Reifen $\mathrm{GmbH}$ in Hannover. 



\section{4 Entwicklung und Synthese funktionalisierter RAFT-Agenzien}

Bei der RAFT-Agens-Synthese wurden verschiedene Strategien verfolgt. Das RAFTAgens soll die Polymerisation von Butadien hinsichtlich einheitlichem Kettenwachstum zuverlässig kontrollieren. Es bietet sich an, eine bewährte Z-Gruppe zu wählen und die R-Gruppe nach den eigenen Bedürfnissen zu modifizieren. Nach Schema 2.11 eignen sich für Styrol sowohl Dithiobenzoate $(Z=P h)^{[74]}$ als auch Trithiocarbonate ( $\mathrm{Z}=\mathrm{S}$-Alkyl). Für die R-Gruppe haben sich in Butadien-Polymerisationen tertiäre Abgangsgruppen und sekundäre Abgangsgruppe mit einer zusätzlichen Stabilisierung (Phenyl- oder Nitrilrest) bewährt. ${ }^{[23,75,76]}$ Dies sind beides sterisch anspruchsvolle Gruppen, was die Anknüpfung über Substitutionsreaktionen erschwert. Bei dem Sonderfall der symmetrischen Trithiocarbonate ist die Abgangsgruppe identisch mit dem Alkylrest der Z-Gruppe.

In diese Abgangsgruppe muss eine der beiden funktionellen Gruppen (siehe Schema 3.1) eingebaut werden, was eine mindestens zweistufige Synthese bedingt. Im ersten Schritt wird die Abgangsgruppe synthetisiert, die im zweiten Schritt, der RAFTAgens-Synthese, angekoppelt wird. Eventuell ist ein weiterer Schritt notwendig, in dem eine Vorstufe des RAFT-Agens oder die Funktionalität in einer reaktiven, verar- 
beitbaren Form synthetisiert wird.

Symmetrische Trithiocarbonate haben den Vorteil, dass sie an beiden Enden die gewünschte Funktionalität tragen und somit den Einbau der Kettenenden in das Polymernetzwerk unterstützen. Es ist möglich, aber noch nicht erwiesen, dass RAFT-Agenzien während der Vulkanisation gespalten werden und ein endständiges Thiol übrig bleibt, welches einvulkanisiert werden kann.

\subsection{Forschungsstand}

Die RAFT-Agenzien sollen, sofern sie sich in einer Butadien-Polymerisation bewährt haben, im großen Maßstab hergestellt und eingesetzt werden können. Dies setzt eine einfache und kostengünstige Synthese voraus. Reaktionen, die eine Ausbeute von $50 \%$ oder weniger liefern und eine mehrstufige Synthese mit säulenchromatographischer Aufreinigung nach jeder Stufe erfordern, sind nur zu Forschungszwecken geeignet.

Es haben sich inzwischen für die unterschiedlichen Z-Gruppen verschiedene Synthesen bewährt, die wiederum verschiedene Reagenzien für die R-Gruppen-Anknüpfung bedingen. Im Folgenden werden literaturbekannte Ansätze und daraus abgeleitete Strategien vorgestellt. Bei verallgemeinerten Reaktionsgleichungen wird das in der organischen Chemie geläufige " $\mathrm{R}^{\prime \prime}$ als Abkürzung für organische Reste beibehalten. Um Verwechslungen mit der R-Gruppe der RAFT-Agenzien zu vermeiden, sind die Alkylbzw. Arylreste stets mit einer Ziffer versehen; auch dann, wenn in einer Reaktion nur ein Rest vorkommt und eine Nummerierung überflüssig scheint. Gleiche Ziffern in unterschiedlichen Reaktionsgleichungen entsprechen nicht zwangsweise den gleichen Resten, schließen dies aber auch nicht aus. Näheres ist der Primärquelle zu entnehmen.

\subsubsection{Dithiobenzoate}

Dithiobenzoate wurden, ebenso wie Trithiocarbonate, schon deutlich vor Entdeckung der RAFT-Polymerisation synthetisiert. Bereits im Jahr 1973 veröffentlichten Meijer et al. eine Dithioestersynthese., ${ }^{[77]}$ in der sie unterschiedliche Alkyl- und Arylhalogenverbindungen (darunter Brombenzol) mit Magnesium zu einem Grignard-Reagenz reagieren lassen. Nach Schwefelkohlenstoff-Zugabe wird das Dithiobenzoat gebildet, welches bei saurer Aufarbeitung Dithiobenzoesäue liefert. Wird statt einer Säure eine pri- 
märe $\left(\mathrm{R}^{2}=\mathrm{H}\right)$ oder sekundäre Halogenverbindung zugegeben, reagiert Natriumdithiobenzoat in einer nukleophilen Substitution zu dem erwünschten RAFT-Agenz. Bei tertiären Halogenverbindungen oder sekundären mit sterisch anspruchsvollen Resten verläuft diese Reaktion mit schlechteren Ausbeuten als bei primären oder sekundären Halogenverbindungen.

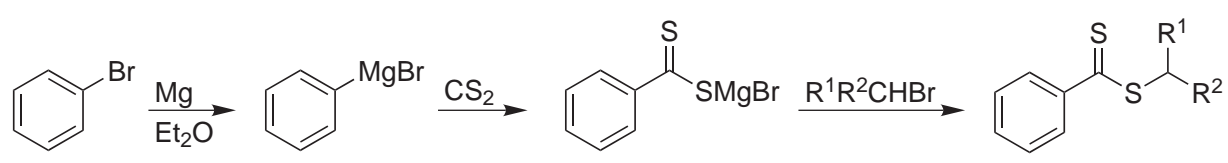

Schema 4.1 Dithiobenzoat-Synthese mit der Grignard-Reaktion. ${ }^{[12,77,78]}$

Das zu substituierende Halogen ist i. d. R. Brom, da es eine große Auswahl kommerziell verfügbarer Bromverbindungen gibt und Brom eine gute Abgangsgruppe in einer nukleophilen Substitution ist. Chlor ist eine schlechtere Abgangsgruppe, stellt aber trotzdem oft eine brauchbare Alternative dar, wohingegen bei dem reaktiveren Iod die Verfügbarkeit stark eingeschränkt ist. Die Ausbeuten liegen bei $\geq 70 \%$ für primäre und sekundäre Halogenverbindungen und bei 5 - 40 \% für tertiäre Halogenverbindungen. ${ }^{[12]}$ Für sterisch anspruchsvolle Gruppen bietet sich die Reaktion von Olefinen mit Dithiobenzoesäure an. Aus Benzylchlorid entsteht in einer Reaktion mit elementarem Schwefel und Natriummethanolat Natriumdithiobenzoat, welches nach saurer Aufarbeitung Dithiobenzoesäure liefert ${ }^{[79]}$ (siehe Schema 4.2; alternativ in einer GrignardReaktion mit Schwefelkohlenstoff und anschließender saurer Aufarbeitung, ${ }^{[80]}$ siehe Schema 4.1). Diese kann in einer Umlagerungsreaktion in einem unpolaren Lösungsmittel wie Hexan oder Tetrachlorkohlenstoff mit einem Olefin reagieren und liefert abhängig vom Substituenten eine sekundäre $\left(\mathrm{R}^{3}=\mathrm{H}\right)$ oder tertiäre Abgangsgruppe. ${ }^{[40]}$

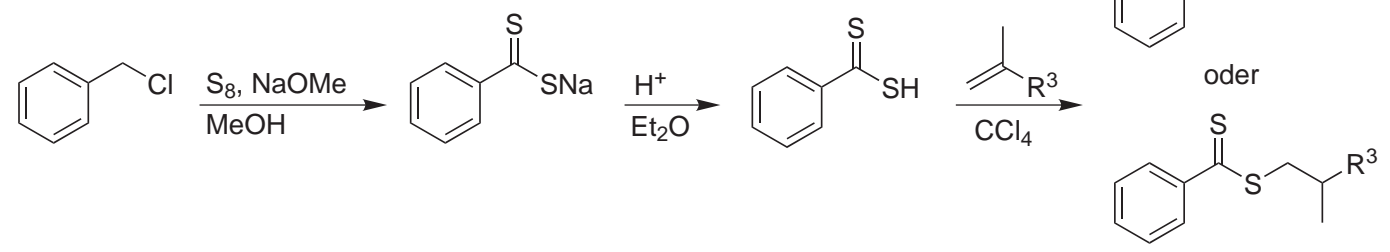

Schema 4.2 Dithiobenzoat-Synthese mit elementarem Schwefel. ${ }^{[12,77]}$

Wichtig ist hierbei die elektronische Struktur des Olefins. Während elektronenreiche Olefine wie Styrole oder $\alpha$-Methylstyrole das erwünschte Produkt liefern (Schema 4.2, oben), reagieren elektronenarme Olefine (z. B. Acrylate und Methacrylate) bevorzugt 
in einer Michael-ähnlichen Addition. Dabei bindet der Schwefel an die unsubstituierte Position und es wird die unerwünschte primäre Abgangsgruppe gebildet (Schema 4.2, unten). ${ }^{[12]}$

Eine weitere beliebte Methode wurde von Thang et al. im Jahr 1999 publiziert und beruht auf der Synthese von Disulfiden, die radikalisch mit einer Azoverbindung wie AIBN zu dem gewünschten Produkt umgesetzt werden ${ }^{[81]}$ (Schema 4.3). Dies geschieht in einer inerten Atmosphäre bei Erhitzen unter Rückfluss innerhalb von vier bis fünf Halbwertszeiten der Azoverbindung. Dabei zerfällt die Azoverbindung, Stickstoff wird frei und die entstehenden Radikale können das Disulfid spalten und zu dem RAFTReagenz reagieren. So können auch Agenzien mit tertiären Abgangsgruppen in hohen Ausbeute erreicht werden, was bei den anderen Reaktionen, die häufig nach dem $\mathrm{S}_{\mathrm{N}} 2$ Mechanismus ablaufen, aus sterischen Gründen nicht möglich ist. Der Nachteil liegt darin, dass eine geeignete Azoverbindung vorhanden sein muss, was häufig nicht der Fall ist.

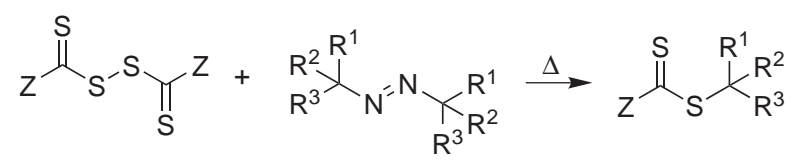

Schema 4.3 Dithioester-Synthese mit Hilfe von Azoverbindungen. ${ }^{[81]}$

Darüber hinaus besteht die Möglichkeit, Thioester zu Dithioestern umzusetzen. Dies geschieht mit dem sogenannten Lawesson-Reagenz, einem hypervalenten, schwefelübertragenden Phosphorreagenz, dass aufgrund der Oxophilie des Phosphors das Carbonyl-Sauerstoffatom des Edukts abstrahiert und durch ein Schwefelatom ersetzt (Schema 4.4). ${ }^{\left[{ }^{[2]}\right.}$ Dies setzt jedoch eine gute Zugänglichkeit des Thioesters voraus, die in vielen Fällen nicht gegeben ist.
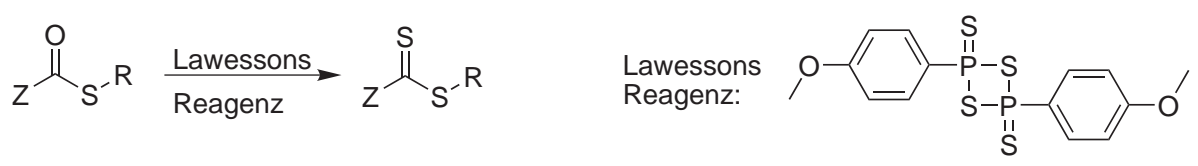

Schema 4.4 Dithioester-Synthese mit Hilfe des Lawesson-Reagenzes. ${ }^{[82]}$ 


\subsubsection{Trithiocarbonate}

Eine Übersicht über unterschiedliche Synthesemöglichkeiten für Trithiocarbonate wurde erstmals von Godt und Wann im Jahr 1961 veröffentlicht ${ }^{[83]}$ (siehe Schema 4.5). Sie skizzieren drei Synthesemöglichkeiten für asymmetrische Trithiocarbonate sowie eine für symmetrische Trithiocarbonate. Ein Thiol kann unter basischen Bedingungen mit einem Chlordithioformiat reagieren und das gewünschte Trithiocarbonat bilden, was allerdings die Synthese von Chlordithioformat als Vorstufe vorraussetzt (diese findet sich z. B. bei Theis et al. ${ }^{[84]}$ ). Des Weiteren kann ein Thiol unter basischen Bedingungen deprotoniert werden und bei anschließender Schwefelkohlenstoff-Zugabe wird das Trithiocarbonat gebildet. Dieses kann bei Organohalogen-Zugabe oder mit Alkyl- bzw. Aryldiazoniumchlorid zu dem gewünschten Produkt reagieren. Symmetrische Trithiocarbonate können in der Reaktion von Thiophosgen mit zwei Äquivalenten des Thiols (plus evtl. Überschuss) unter basischen Bedingungen gebildet werden.

Die Umsetzung von Thiolen mit Thiophosgen zu symmetrischen Trithiocarbonaten sowie die Deprotonierung eines Thiols mit einer Base und anschließende Umsetzung mit einem Halogenalkan zu einem asymmetrischen Trithiocarbonat findet sich ebenfalls in der von Barton und Ollis im Jahr 1979 herausgegebenen Enzyklopädie „Comprehensive Organic Chemistry“. ${ }^{[85]}$

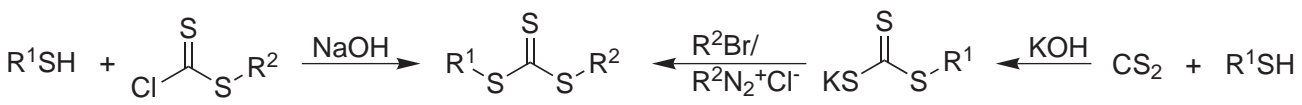

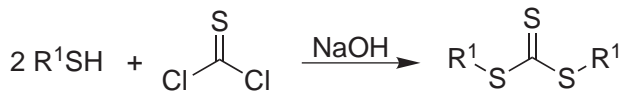

Schema 4.5 Unterschiedliche Synthesemöglichkeiten für Trithiocarbonate nach Godt und Wann. ${ }^{[83]}$

Da Trithiocarbonate vielfältig eingesetzt werden können (z. B. als Pestizide in der Landwirtschaft sowie als Schmiermitteladditive ${ }^{[86]}$ ), war bereits in den 1980er Jahren, also noch vor der Entwicklung der RAFT-Polymerisation, das Bestreben groß, das Repertoire der Reaktionen und Substanzen zu vergrößern. In den späten 1960er Jahren wurde die Phasentransferkatalyse von verschiedenen Arbeitsgruppen unabhängig voneinander entwickelt. Bekannt geworden sind vor allem die Arbeiten von Starks, nach dem ein beliebter Phasentransferkatalysator, Tricaprylmethylammoniumchlorid, kommerziell unter dem Namen Aliquat ${ }^{\circledR} 336$ erhältlich, benannt ist (Starks-Katalysator). Bei Phasentransferkatalysatoren handelt es sich um quartäre Phosphonium- oder Ammo- 
niumsalze, welche langkettige Alkylsubstituenten tragen. Diese verleihen der Substanz einen lipophilen Charakter, während die ionische Ladung ihr hydrophile Eigenschaften gibt. Der genaue Mechanismus wird in Abschnitt 5.3 erklärt. Mit diesen Reagenzien ließen sich auch in der Trithiocarbonatsynthese neue Ergebnisse erzielen.

Im Jahr 1984 veröffentlichten Sugawara et al. eine Synthese für Alkylaryltrithiocarbonate, bei der aus Benzolthiol, Schwefelkohlenstoff und wässriger Natriumhydroxidlösung mit Aliquat ${ }^{\circledR} 336$ das Benzoltrithiocarbonat-Anion gebildet wird, das mit einem Halogenalkan bei Raumtemperatur innerhalb weniger Stunden zu dem gewünschten Endprodukt reagiert (Schema 4.6). ${ }^{[87]}$ Als Nebenprodukt entsteht ein Thioether. Während bei dieser Vorschrift Schwefelkohlenstoff nur im einfachen Überschuss eingesetzt wird, wird mit Benzol als Lösungsmittel für das Halogenalkan dennoch ein sehr giftiges Lösungsmittel verwendet. Ein Austausch gegen Toluol ist vermutlich problemlos möglich und beim heutigen toxikologischen Kenntnisstand angebracht. Abhängig von den Subsitutenten können Umsätze bis zu 85 \% erreicht werden.

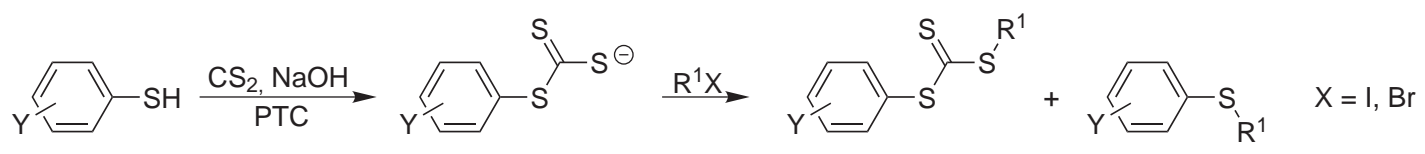

Schema 4.6 Phasentransferkatalysierte Synthese von asymmetrischen Trithiocarbonaten nach Sugawara et al. ${ }^{[87]}$ PTC steht für "phase transfer catalyst".

Während sich Sugawara et al. auf asymmetrische Arylalkyltrithiocarbonate beschränken, stellen Degani et al. im Jahr 1986 eine phasentransferkatalysierte Synthese von asymmetrischen und symmetrischen Trithiocarbonaten vor. ${ }^{[86]}$ Neben Aliquat ${ }^{\circledR}$ 336 werden Tetrabutylammoniumbromid und Hexadecyltributylphosphoniumbromid verwendet sowie die unterschiedlichen Halogene hinsichtlich ihrer Abgangsgruppenqualität untersucht. Die Synthese asymmetrischer Trithiocarbonate erfolgt analog zu Sugawara et al., allerdings bei erhöhter Temperatur $\left(70^{\circ} \mathrm{C}\right.$ statt Raumtemperatur), wohingegen die Synthese symmetrischer Trithiocarbonate neu ist. Diese wird in wässrigem Medium durchgeführt und benötigt nur stöchiometrische Mengen Schwefelkohlenstoff. Das tiefrote Trithiocarbonatanion ${ }^{[88]}$ wird aus Natriumsulfid und Schwefelkohlenstoff mit Hilfe eines Phasentransferkatalysators bei Raumtemperatur in wässriger Lösung gebildet. Die Umsetzung mit dem Halogenalkan in stöchiometrischer Menge erfolgt anschließend bei $70^{\circ} \mathrm{C}$ (siehe Schema 4.7).

Lee et al. publizierten im Jahr 1988 eine phasentransferkatalysierte Synthese von symmetrischen Trithiocarbonaten, die der von Degani et al. ähnelt. Der Unterschied liegt in dem Gegenion des verwendeten Phasentransferkatalysators (hier: Tetrabutylammo- 
niumsulfat) und in der Reaktionstemperatur (hier: Raumtemperatur). ${ }^{[89]}$ Des Weiteren wird wie bei Degani et al., im Gegensatz zu Sugawara et al., auf die Zugabe eines organischen Lösungsmittels verzichtet und die organische Phase wird allein durch die Reaktanden gebildet.

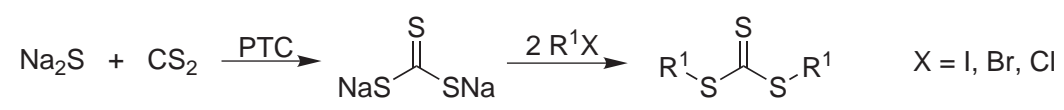

Schema 4.7 Phasentransferkatalysierte Synthese von symmetrischen Trithiocarbonaten nach Degani et al. ${ }^{[86]}$

Eine Neuerung wurde im Jahr 1998 von Tamami und Kiasat vorgestellt: Sie entwickelten eine Synthese für symmetrische Trithiocarbonate unter Zuhilfenahme von Amberlyst ${ }^{\circledR}$ A-26, einem polymerbasierten Katalysator. Diese bestehen aus einem vernetzten Styrol-Divinylbenzol-Copolymer, das an der Oberfläche TrimethylammoniumFunktionalitäten mit Hydroxid als Gegenion tragen (Schema 4.8). ${ }^{90]}$ Es handelt sich dabei im Prinzip um einen festphasengebundenen Phasentransferkatalysator, der den Vorteil besitzt, dass er durch Filtration entfernt werden kann und nicht im Reaktionsgemisch verbleibt (alternativ kann auch Ambersep ${ }^{\circledR} 900 \mathrm{OH}$ verwendet werden, ein Anionenaustauscherharz, das ähnlich aufgebaut ist). Die Reaktion findet in Schwefelkohlenstoff als Lösungsmittel statt. An der Oberfläche des Amberlyst ${ }^{\circledR}$ A-26 bildet sich das tiefrote Trithiocarbonat-Anion, welches beim Zutropfen der halogenorganischen Verbindung mit dieser zu dem gewünschten Trithiocarbonat (i. d. R. gelb) reagiert. Eine säulenchromatographische Aufreinigung ist somit in vielen Fällen überflüssig, sofern sich die Reaktanden vollständig umgesetzt haben.

$$
\begin{aligned}
& 2 \mathrm{P}^{+}(\mathrm{OH})^{-} \stackrel{\mathrm{CS}_{2}}{\longrightarrow} 2 \mathrm{P}^{+}\left(\mathrm{CS}_{3}\right)^{2-} \stackrel{\mathrm{R}^{1} \mathrm{X}}{\longrightarrow} \mathrm{R}^{1} \mathrm{~S}_{\mathrm{S}^{-}}^{\mathrm{H}^{1}+2 \mathrm{P}^{+}(\mathrm{X})^{-}} \quad \mathrm{X}=\mathrm{I}, \mathrm{Br}, \mathrm{Cl}
\end{aligned}
$$

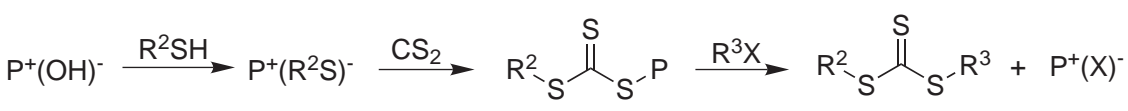

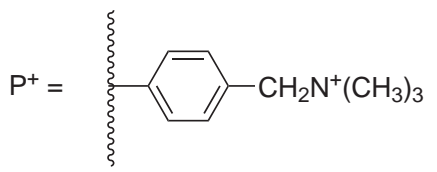

Schema 4.8 Trithiocarbonat-Synthese mit Amberlyst ${ }^{\circledR}$ A-26 nach Tamami und Kiasat. ${ }^{[90,91]}$

Anschließend wurde die Reaktion von derselben Arbeitsgruppe weiterentwickelt, so 
dass auch die Synthese asymmetrischer Trithiocarbonate möglich ist. ${ }^{[91]}$ Dazu werden zuerst Thiole mit Amberlyst ${ }^{\circledR}$ A-26 in einem adäquaten Lösungsmittel (z. B. THF) gerührt. Dabei bildet sich katalysatorgebundenes Thiolat, das abfiltriert, gewaschen und getrocknet werden kann. In einer Schwefelkohlenstoffsuspension bildet sich aus dem Thiolat das entsprechende Trithiocarbonatanion, welches bei Zugabe einer halogenorganischen Verbindung das asymmetrisch substituierte Trithiocarbonat bildet, das in Lösung vorliegt. Symmetrische Trithiocarbonate sind ebenfalls zugänglich, sofern die entsprechenden Thiole und Halogenverbindungen verfügbar sind.

Eine letzte Weiterentwicklung auf dem Gebiet der phasentransferkatalysierten Trithiocarbonatsynthse gelang Kiasat und Mehrjardi im Jahr 2008, die mit Tetrabutylammoniumbromid als Phasentransferkatalysator und Aluminiumoxid in einer EintopfReaktion in Suspension gute Ergebnisse bei der Synthese symmetrischer Trithiocarbonate erzielen (siehe Schema 4.9). ${ }^{[92]}$ Der Einsatz von Tosylaten anstelle von Halogenalkanen ermöglicht z. B. die Synthese von Dimethyltrithiocarbonat ohne den Einsatz des toxischen Methyliodids. Darüber hinaus können basenempfindliche Substanzen umgesetzt werden.

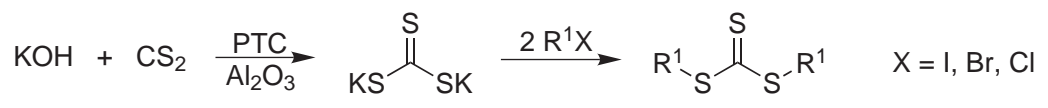

Schema 4.9 Trithiocarbonat-Synthese nach Kiasat und Mehrjardi. ${ }^{[92]}$

Einen weiteren Durchbruch erreichten Aoyagi et al., die im Jahr 2006 eine Synthese veröffentlichten, die sie selbst als „mild and efficient" beschreiben. Bei Raumtemperatur unter schwach basischen Bedingungen, die durch den Einsatz von Cäsiumcarbonat erzeugt werden, wurde Benzylchlorid mit stöchiometrischen Mengen Schwefelkohlenstoff umgesetzt (Schema 4.10). Die Umsetzung erfolgt in Acetonitril, $\mathrm{N}, \mathrm{N}$ Dimethylformamid und $\mathrm{N}, \mathrm{N}$-Dimethylacetamid quantitativ und anschließende Umkristallisation liefert reines Produkt. ${ }^{[93]}$ Weitere Experimente mit anderen Alkylhalogenverbindungen liefern ebenfalls gute Ergebnisse. Als Abgangsgruppe kann neben Brom auch Chlor verwendet werden, was jedoch zu niedrigeren Ausbeuten führt. Während Lithium- und Natriumcarbonat keine ausreichende Basizität haben, können mit Kaliumcarbonat als Base ebenfalls gute Ergebnisse erreicht werden, was jedoch in vielen Fällen eine Erwärmung auf $40^{\circ} \mathrm{C}$ erfordert. Somit liefert Cäsiumcarbonat die besten Ergebnisse unter milden Bedingungen. ${ }^{[94]}$ Der Einsatz von Cäsiumcarbonat zur Bildung des Trithiocarbonatanions läuft unter wasserfreien Bedingungen ab und der Einsatz einer stöchiometrischen Menge Schwefelkohlenstoff macht diese Reaktion auch 
aus toxikologischer Sicht interessant.

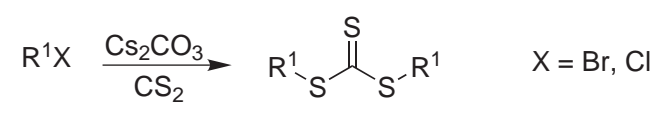

Schema 4.10 Trithiocarbonat-Synthese nach Aoyagi. ${ }^{[93,94]}$

Neben Natrium- und Kaliumhydroxid ist heute Triethylamin als Base zur Deprotonierung des Thiols bei der Trithiocarbonatsynthese gebräuchlich, da es als organische Base in gängigen organischen Lösungsmitteln löslich ist und die wässrige Phase sowie den Einsatz eines Phasentransferkatalysators überflüssig macht. ${ }^{\text {95] }}$

\subsubsection{Fazit und Konsequenzen für die Synthese neuer RAFT-Agenzien}

Jede der Syntheserouten hat Vor- und Nachteile. Während das Arbeiten in wässrigem Medium aufgrund fehlender hydrolyseempfindlicher funktioneller Gruppen kein Problem darstellt, sollte hingegen auf toxische Chemikalien soweit möglich verzichtet werden. Chemikalien mit unangenehmem Geruch müssen vermieden werden, da diese in dem Endprodukt verbleiben und es somit für einen Einsatz in der Industrie unbrauchbar machen. Inertes Arbeiten ist möglich, macht den Prozess aber arbeits- und somit kostenintensiver und sollte daher vermieden werden. Verunreinigungen in Form eines Phasentransferkatalysators oder des Edukts sollten entfernt werden, sofern sie die Polymerisation beeinflussen. Ist dies nicht der Fall, können kleine Mengen toleriert werden, da die Weiterverarbeitung nicht komplett chemisch rein erfolgt. Geringe Ausbeuten stellen bei der Weiterentwicklung zur Produktreife ein Problem dar, können aber im Labormaßstab toleriert werden. Das Hauptproblem liegt in der Verfügbarkeit der benötigten Reagenzien, die von Reaktion zu Reaktion variiert.

Ein Großteil der Reaktionen beruht auf dem Mechanismus der Nukleophilen Substitution und benötigt eine geeignete Abgangsgruppe. Dabei spielt sowohl die Stärke der Bindung eine Rolle, als auch die Polarisierbarkeit dieser Bindung. Daraus ergibt sich, dass bei den Halogeniden die Reaktivität von Iodid zu Fluorid abnimmt. Da Iodverbindungen in den seltensten Fällen zur Verfügung stehen, wird auf Brom- oder Chlorreagenzien zurückgegriffen. Bromverbindungen sind in großer Auswahl kommerziell erhältlich, allerdings sind Chlorverbindungen oft preiswerter als die reaktiveren Bromanaloga. Es ist daher individuell abzuwägen, welchem der Vorzug zu geben ist. 


\subsection{Synthese funktionalisierter Anker}

$\mathrm{Zu}$ Anbindung der potenziell vulkanisierbaren Funktionalitäten wurde auf die Synthese von sog. Ankern zurückgegriffen. Dies sind Moleküle, welche die funktionelle Gruppe 1 oder 2 tragen und darüber hinaus eine Möglichkeit bieten, an ein RAFTAgens angebunden zu werden (z.B. ein Halogenatom, eine Alkoholfunktion oder eine Doppelbindung). Die Anker, die im Rahmen dieser Arbeit synthetisiert und verwendet wurden, sind in Schema 4.11 dargestellt. Sie tragen alle eine Thioacetat-Funktion, da diese sich leichter anbinden ließ als die Benzolthiosulfonat-Funktion, die aufgrund der ionischen Struktur Löslichkeitsprobleme mit sich brachte. Die Anbindung von Thioacetat kann auf unterschiedliche Arten erfolgen. Ein Ester lässt sich durch die Veresterung eines Alkohols mit einer Carbonsäure synthetisieren, wobei das verbrückende Atom aus dem Alkohol stammt, so dass für die Synthese von Thioessigsäureestern ein Thiol mit einer Carbonsäure umgesetzt werden muss. Damit ein für Butadien geeignetes RAFT-Agens gebildet werden kann, muss ein Halogen in sekundärer oder tertiärer Position vorhanden sein oder eine nicht-terminale Doppelbindung. Da Thiole nur in einer begrenzten Auswahl kommerziell verfügbar sind, die Synthesen in schlechten Ausbeuten verlaufen und einen weiteren Schritt in der ohnehin mehrstufigen Synthese bedeuten und für die Anwendung in technischen Produkten aufgrund ihres Geruchs nur bedingt geeignet sind, wurde auf die direkte Veresterung verzichtet.

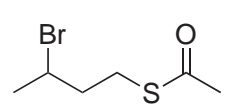

8<smiles>CC(=O)SCCCO</smiles>

9<smiles>CC(=O)SCCCCl</smiles>

10

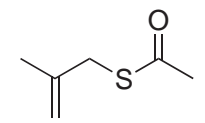

11

Schema 4.11 Verwendete Anker mit Thioacetat-Funktion.

Des Weiteren gibt es die Möglichkeit, Thioessigsäure mit einer schwachen Base wie Kaliumcarbonat zu deprotonieren und mit einem Halogenalkan umzusetzen. ${ }^{[96]}$ Unter diesen milden Reaktionsbedingungen werden auch andere funktionelle Gruppen i. d. R. nicht angegriffen.

Darüber hinaus gibt es die Möglichkeit, für die Synthese von Thioessigsäureestern Kaliumthioacetat einzusetzen, welches mit einem Halogen-substituierten Reagenz bereits bei Raumtemperatur in moderaten Ausbeuten zu dem gewünschten Ester reagiert. ${ }^{[97,98]}$

Die Synthesen sind in Schema 4.12 schematisch dargestellt. Für die Anker 8, 10 und 

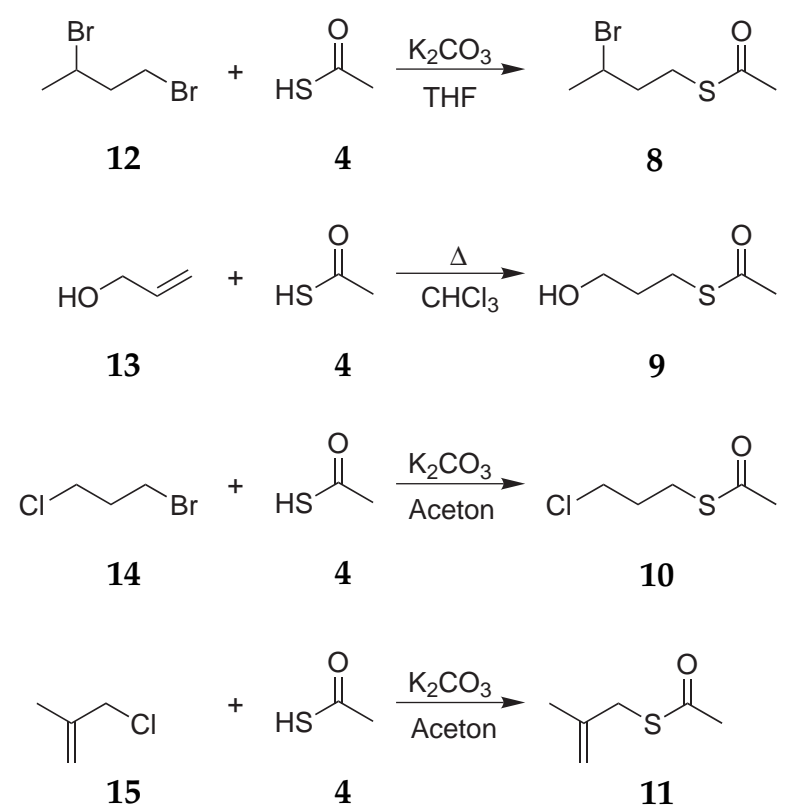

Schema 4.12 Synthese der verwendeten Anker mit Thioacetat-Funktion.

11 wurde Thioessigsäure 4 mit 1,3-Dibrompropan 12, 1-Brom-3-chlorpropan 14 bzw. 1-Chlor-2-methylprop-2-en 15 unter schwach basischen Bedingungen in einem polaraprotischen Lösungsmittel umgesetzt. In der Synthese von 9 reagierte Allylalkohol 13 mit Thioessigsäure 4 unter Erhitzen.

8 wurde in der Synthese eines Trithiocarbonats eingesetzt, welche in Kapitel 4.3 vorgestellt wird. 9 wurde zur Synthese eines makromolekularen RAFT-Agens eingesetzt, welche in Kapitel 4.4 vorgestellt wird.

Die Anker 10 und 11 konnten nicht erfolgreich verwendet werden. Bei 10 ist vermutlich das Chloratom eine zu schlechte Abgangsgruppe und ein Ausweichen auf Brom unumgänglich. Da bei der Synthese des entsprechenden Brom-Derivates aus 1,3Dibrompropan das disubstituierte Alkan gebildet wird, welches einen weiteren Aufreinigungsschritt erfordert und zu Umsatzeinbußen führt, wurde der Ansatz nicht weiter verfolgt.

Die Anbindung von 11 an Dithiobenzoesäure konnte ebenfalls nicht erfolgreich durchgeführt werden. Darüber hinaus ist es wahrscheinlich, dass dieser Anker nicht das gewünschte Produkt geliefert hätte, da es sich um ein relativ elektronenarmes Olefin handelt, das zur Bildung des unerwünschten Nebenproduktes aus Schema 4.2, unten, neigt. Somit würde das RAFT-Agens keine sekundäre Abgangsgruppe tragen, wie es für eine Butadien-Polymerisation vorteilhaft wäre, sondern eine primäre Abgangsgruppe. 


\subsection{Trithiocarbonat mit Thioacetat-Funktionalität}

Da es sich bei dem Anker 8 um eine sekundäre Halogenverbindung handelt, ist die Wahrscheinlichkeit hoch, dass sich ein RAFT-Agens mit dieser Abgangsgruppe in einer Butadien-Polymerisation bewährt, sofern die mangelnde Stabilisierung durch einen Phenylrest oder einen anderen elektronenschiebenden Substituenten die Radikalstabilität nicht zu weit absenkt.

Es wurde das symmetrisches Trithiocarbonat 16 synthetisiert (Schema 4.13), da dieses eine Funktionalisierung beider Polymerkettenenden gewährleistet. Dabei wurde die von Aoyagi et al. entwickelte Reaktion mit Cäsiumcarbonat als Base gewählt, da diese erfahrungsgemäß für sekundäre halogenorganische Verbindungen mit höchstens einem sterisch anspruchsvollen Substituenten gute Ausbeuten liefert.

Das RAFT-Agens wurde in einer Styrol-Polymerisation eingesetzt, um die Verwendbarkeit zu untersuchen. Die Ergebnisse werden in Kapitel 6.1 vorgestellt.

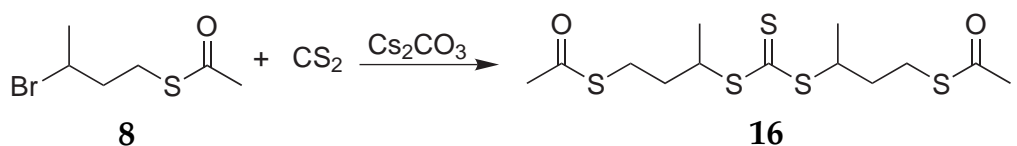

Schema 4.13 Synthese des RAFT-Agens.

\subsection{Makro-RAFT-Agens mit Thioacetat-Funktionalität}

Ein ungewöhnlicher neuer Ansatz wurde in unserer Arbeitsgruppe entwickelt, ${ }^{[99]}$ bei dem in einer Eintopf-"Klick"-Reaktion Alkohole mit Aziden reagieren. Ausgangspunkt ist ein Trithiocarbonat mit endständiger Carboxylgruppe in sterisch anspruchsvoller Umgebung (Schema 4.14, 17). Es kann in einer einstufigen Reaktion selbst synthetisiert werden ${ }^{[100,101]}$ und ist inzwischen auch kommerziell erhältlich. Dieses RAFT-Agenz, ein gelber Feststoff, wird nach Deptrotonierung mit Triethylamin durch Zugabe von Diphenylphosphorylazid zum Carbonylazid 18 umgewandelt (siehe Schema 4.14).

Unter normalen Umständen würde dies zu einem Isocyanat $(-\mathrm{N}=\mathrm{C}=\mathrm{O})$ umlagern. Aufgrund der beiden Methylgruppen in Nachbarschaft zum Carbonylkohlenstoffatom ist diese Umlagerung sterisch jedoch nicht möglich und das RAFT-Agens ist selbst bei Temperaturen von $35-90{ }^{\circ} \mathrm{C}$ stabil. ${ }^{[99]}$ 


$$
\begin{array}{ll}
\text { 1) } \mathrm{Et}_{3} \mathrm{~N} \\
\text { 2) }(\mathrm{PhO})_{2} \mathrm{P}(\mathrm{O}) \mathrm{N}_{3} \text { in } \mathrm{CH}_{2} \mathrm{Cl}_{2}
\end{array}
$$

17

Schema 4.14 Synthese des RAFT-Agens 18 $^{[99]}$ sowie der Vorstufe 17. ${ }^{[100,101]}$

Wird allerdings in einer Polymerisation mit einem für Trithiocarbonate geeigneten Monomer (z. B. NIPAM, MA, MMA oder Styrol) diese sterische Hinderung aufgehoben, kann es zu einer Umlagerung kommen. In der Anwesenheit eines Alkohols (und einer katalytischen Mengen von Dibutylzinndilaurat, DBZDL) reagiert dieser mit dem Azid und es bildet sich ein Carbamat. Gody et al. zeigten dies mit dem RAFT-Agens 18 und TMS-Propargylalkohol. TMS-Propargylalkohol wurde als Modellverbindung gewählt, da die chemische Verschiebung der TMS-Methylgruppen sehr charakteristisch ist und mit keinem anderen Signal überlagert. In dieser Arbeit wurde stattdessen ein Alkohol verwendet, der eine Thioacetat-Endgruppe trägt, welche im ${ }^{13} \mathrm{C}-\mathrm{NMR}$ ein charakteristisches Signal liefert ( $\delta \approx 195 \mathrm{ppm}$ ). Die Polymerisation des Azids 18 mit Methylacrylat zur Anbindung des Alkohols sowie eine Styrol-Polymerisation mit dem daraus gewonnenen Makro-RAFT-Agens wird in Kapitel 6.2 vorgestellt.

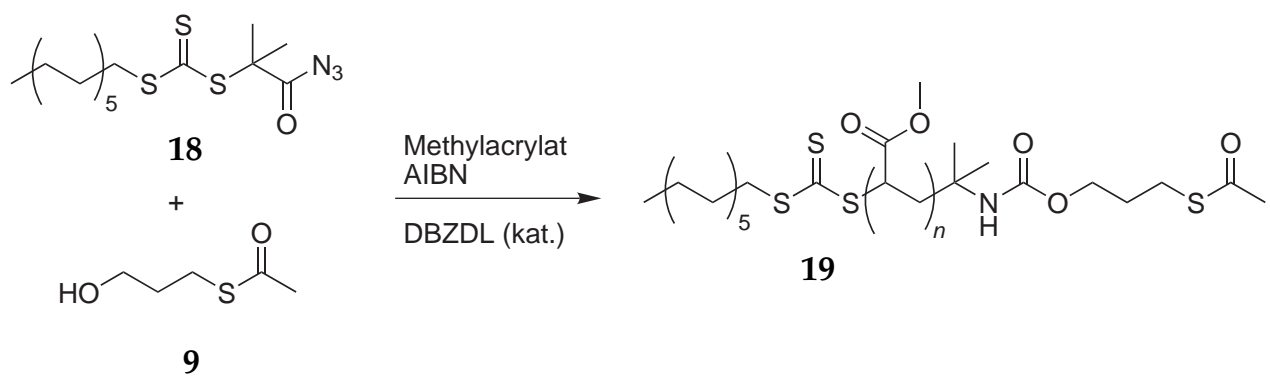

Schema 4.15 Synthese des Makro-RAFT-Agenzes $19,{ }^{[99]} \mathrm{n} \approx 15$. 



\section{Entwicklung und Synthese funktionalisierter Monomere}

Bei der Monomersynthese wurden unterschiedliche Strategien verfolgt, bei denen verschiedene Parameter eine Rolle spielen. Das Monomer muss ein Grundgerüst haben, das sich gut in einer Polymermischung mit Natur- und anderen Synthesekautschuken verarbeiten lässt. Da es sich dabei um Polymere aus sehr unpolaren Monomeren handelt, sollte das funktionalisierte Monomer ebenfalls ein unpolares Gerüst haben. Acrylate kommen somit nicht in Frage. Des weiteren muss die benötigte Vorstufe preiswert in großen Mengen verfügbar sein und die Synthese effizient sein, da bereits im Technikumsmaßstab zur Synthese von 80 - $100 \mathrm{~g}$ Copolymer rund $15 \mathrm{~g}$ des funktionalisierten Monomers oder mehr (je nach Molmasse) benötigt werden.

Es bietet sich an, Styrol-Derivate zu synthetisieren. Styrol hat sich in der Copolymerisation mit Butadien bewährt und bietet darüber hinaus diverse kommerziell verfügbare Derivate, die verwendet werden können. 


\subsection{Copolymerisationsverhalten}

Das Copolymerisationsverhalten zweier Monomere lässt sich am besten anhand empirisch ermittelter Copolymerisationsparameter überprüfen. Wie bereits in Kapitel 2.3 beschrieben, gibt es für eine wachsende Polymerkette in einer Copolymerisation mit zwei Monomeren immer zwei Möglichkeiten (solange die Terminierung vernachlässigt wird): Es kann das Monomer angelagert werden, das gerade auch die Radikalfunktion trägt und somit auch zuletzt angelagert wurde, oder es kann das andere Monomer angelagert werden. Für die Synthese statistischer Copolymere ist ein ausgewogenes Verhältnis, was sich in einerm Copolymerisationsparameter von 1 widerspiegelt, von Vorteil.

Für eine Copolymerisation von Butadien mit Styrol bei $60^{\circ} \mathrm{C}$ betragen die Copolymerisationsparameter 1,39 bzw. 0,78. Abbildung 5.1 zeigt das Copolymerisationsdiagramm für Butadien und Styrol bei $60^{\circ} \mathrm{C}$, was eine typische Polymerisationstemperatur für radikalische, AIBN-initiierte Polymerisationen ist. Aufgetragen ist die Konzentration des Monomers $\mathrm{X}$ im Polymer $[\mathrm{X}(\mathrm{P})]$ gegen die Konzentration von $\mathrm{X}$ in der Monomermischung [X]. Bei idealem Verhalten würde sich die Konzentration in der Monomermischung 1:1 auf die Polymermischung übertragen. Dies ist selten der Fall. Styrol und Butadien stellen jedoch ein sehr ausgewogenes, fast ideales System dar. Für die Funktionalisierung nachteilig ist, dass Butadien im Polymer angereichert wird und somit eine im Verhältnis größere Menge des synthetisierten Comonomers in der Monomermischung benötigt wird, als im Polymer eingebaut ist. Eine Monomermischung, die zu $40 \%$ aus Styrol und zu $60 \%$ aus Butadien besteht, wird ein Polymer mit 33\% Styrolund $67 \%$ Butadienanteil bilden (siehe Abbildung 5.1).

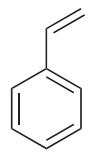

20

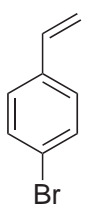

21

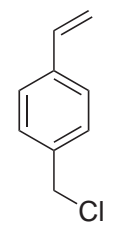

22

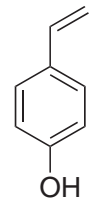

23

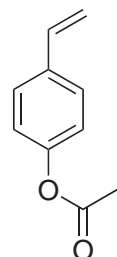

24

Schema 5.1 Styrol 20 sowie die Styrolderivate 4-Bromstyrol 21, 4-Vinylbenzylchlorid 22, 4-Hydroxystyrol 23 sowie 4-Acetoxystyrol 24.

Es wurde ein Anteil von ca. 2 - 5 mol-\% funktionalisiertem Monomer im Copolymer angestrebt. In Schema 5.1 sind unterschiedliche Styrol-Derivate dargestellt, die zur Syn- 
these dieser funktionalisierten Monomere herangezogen werden können. Bei der Benennung wurden die gängigen Bezeichnungen der Hersteller beibehalten, obwohl diese nicht den streng systematischen Bezeichnungen gemäß IUPAC entsprechen.

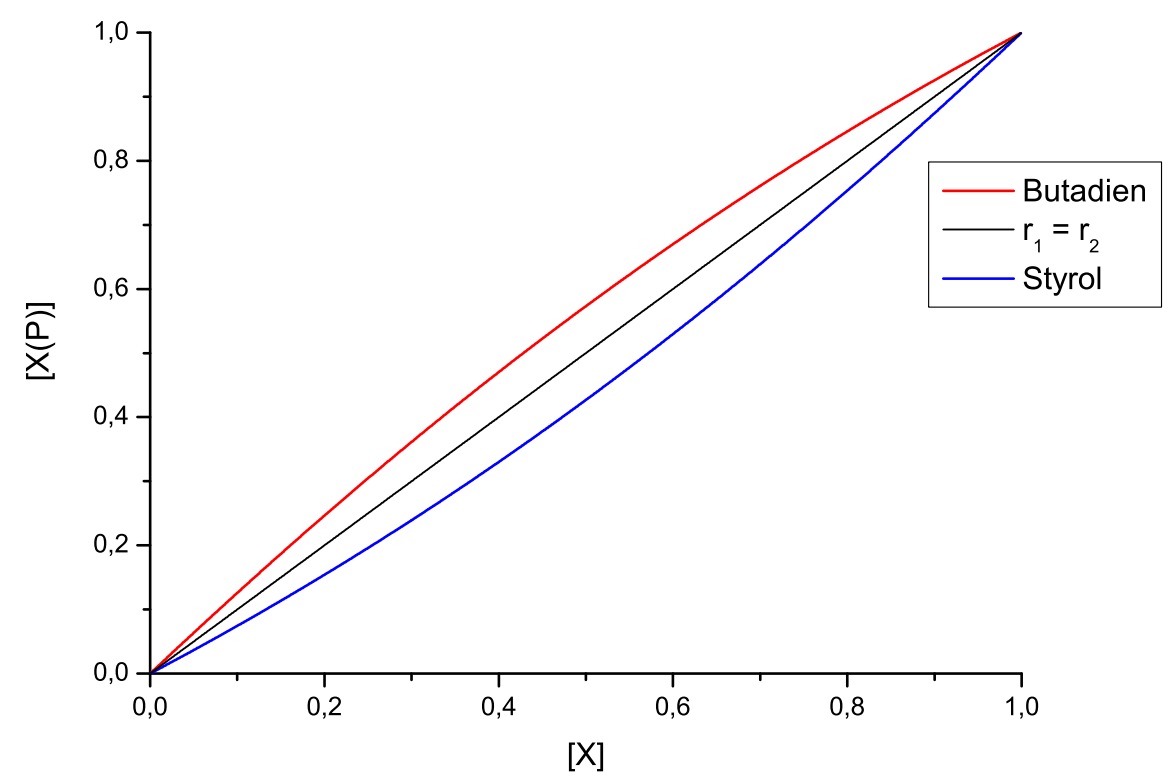

Abbildung 5.1 Copolymerisationsdiagramm für Butadien (Monomer 1) mit Styrol (Monomer 2). Die Copolymerisationsparameter bei $60^{\circ} \mathrm{C}$ sind $\mathrm{r}_{1}=1,39$ und $\mathrm{r}_{2}=0,78 \cdot{ }^{[64]}$

Bei 4-Bromstyrol 21 sowie 4-Vinylbenzylchlorid 22 handelt es sich um potenzielle Reagenzien für eine nukleophile Substitution. Bei 21 würde die funktionelle Gruppe direkt an den Phenylring gebunden sein, was in zweierlei Hinsicht nachteilig ist. Zum einen ist die Substitution direkt am Aromaten sterisch schwierig, zum anderen ist die funktionelle Gruppe selbst sterisch gegen Angriffe geschützt. Da sie während der Vulkanisation einen Netzknotenpunkt ausbilden soll, ist es sinnvoll, sie räumlich von dem Phenylring zu trennen. Während 22 ein weit verbreitetes Monomer ist, ist die entsprechende Bromverbindung kommerziell nur eingeschränkt verfügbar. Es wurde daher auf die Chlorverbindung zurückgegriffen. 4-Hydroxystyrol 23 könnte z. B. mit einer Carbonsäure verestert werden und so ein funktionalisiertes Monomer ergeben. Da es sich hierbei um eine instabile Verbindung handelt, ist sie nicht kommerziell erhältlich. 4-Acetoxystyrol 24 ist ein mit einer Esterschutzgruppe versehenes Äquivalent, welches unter basischen Bedingungen 23 freisetzt. ${ }^{[102,103]}$ Unter Schutzgas bei tiefen Temperaturen lässt sich das so gewonnene Styrolderivat einige Monate lang lagern. 
Ein weiteres Problem stellt die Verfügbarkeit der funktionellen Gruppen dar. Wie Schema 3.2 zeigt, liegt die funktionelle Gruppe 2 in Form des Natriumsalzes 5 vor. Dieses Natriumsalz erfordert ein stark polares Lösungsmittel, während die Styrolderivate sich in unpolaren Lösungsmitteln lösen. Um dieses Problem zu umgehen, wurden Phasentransferkatalysatoren verwendet.

\subsection{Nukleophile Substitution}

Die nukleophile Substitution ist eine weitverbreitete Reaktion zum Austausch von Substituenten in organischen Molekülen. Während bei einer $\mathrm{S}_{\mathrm{N}} 1$-Substitution der geschwindigkeitsbestimmende Schritt, die Abspaltung der Abgangsgruppe, nicht von der Nukleophilie des angreifenden Agens abhängt, nimmt bei einer $S_{N}$ 2-Reaktion die Reaktionsgeschwindigkeit mit der Nukleophilie der angreifenden Verbindung zu. Thiolat-Ionen sind aufgrund der Größe des Schwefelatoms gute Nukleophile und besser geeignet als z. B. Alkoholat. Daneben spielt die Wahl des Lösungsmittels eine entscheidende Rolle. Für $\mathrm{S}_{\mathrm{N}} 1$-Reaktionen werden polar-protische Lösungsmittel verwendet und für $\mathrm{S}_{\mathrm{N}}$ 2-Reaktionen polar-aprotische Lösungsmittel. Ein Wechsel des Lösungsmittels kann mit einer Änderung des Reaktionsmechanismus einhergehen. Die Reaktionen, die im Folgenden beschrieben werden, verlaufen größtenteils nach dem $\mathrm{S}_{\mathrm{N}} 2$ Mechanismus.

\subsubsection{Synthese von 4-Vinylbenzylthioacetat (ViBET)}

Die Synthese von ViBET 25 erfolgte in polar-aprotischen Lösungsmitteln (THF, Aceton) aus 4-Vinylbenzylchlorid 22 und Thioessigsäure 4 unter Zuhilfenahme einer Base (Kaliumcarbonat; siehe Schema 5.2). Die Synthese ist auch für den Technikumsmaßstab geeignet, da sich das Edukt quantitativ umsetzt und keine weitere Aufreinigung erforderlich ist.

Die von Nummy ${ }^{[104]}$ vorgestellte Synthese erfolgt in kurzkettigen aliphatischen Alkoholen wie Methanol mit einem Thiosäuresalz (hergestellt in situ aus Thioessigsäure 4 und einem Alkalimetallhydroxid), welches mit 4-Vinylbenzylchlorid 22 zu dem gewünschten Produkt reagiert, das aber destillativ aufgereinigt werden muss. 


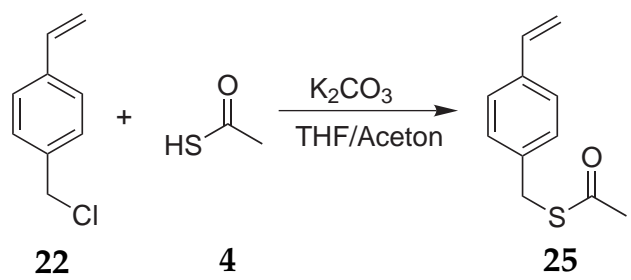

Schema 5.2 Synthese des funktionalisierten Monomers 4-Vinylbenzylthioacetat.

\subsection{Phasentransferkatalyse}

Bei der Phasentransferkatalyse werden Hilfsreagenzien eingesetzt, die ein Molekülfragment von einer Phase in eine andere transferieren. Diese Katalysatoren werden häufig PTC, von dem englischen Wort "phase transfer catalyst", abgekürzt. Pionierarbeit auf dem Gebiet der Phasentransferkatalyse leistete Charles M. Starks, der 1971 in einem Übersichtsartikel die Verwendung von quartären Ammonium- und Phosphoniumsalzen für den Anionentransfer in heterogenen Reaktionen vorstellte. ${ }^{[105]}$ Das Stickstoff- bzw. Phosphoratom dieser quartären Salze trägt vier organische Reste (Alkyl, Allyl, Phenyl oder Benzyl) und ist dadurch positiv geladen. Die positive Ladung wird durch ein Gegenion (z. B. ein Halogenid) ausgeglichen. Somit enthält der Katalysator sowohl einen lipophilen Teil (die organischen Reste) als auch einen hydrophilen Teil (die ionische Ladung). Die Struktur weist Ähnlichkeiten zu typischen Emulgatoren auf, was dazu führt, dass z. B. quartäre Salze mit zwei oder drei Methylgruppen und entsprechend ein bis zwei langkettigen Alkylgruppen zur Ausbildung stabiler Emulsionen führen. Salze mit Allyl-, Phenyl- und Benzylsubstituenten haben sich bei den üblichen Reaktionsbedingungen als instabil herausgestellt. Phosphoniumsalze sind deutlich temperaturstabiler als die korrespondierenden Ammoniumsalze (bis zu $200^{\circ} \mathrm{C}$ bzw. bis zu $100^{\circ} \mathrm{C}$ ), sind aber i. d. R. auch teurer. ${ }^{[105]}$

Das Grundprinzip ist in Schema 5.3 dargestellt. Es findet ein ständiger Austausch zwischen der wässrigen Phase (unten) und der organischen Phase (oben) statt. Eingewogen werden die organische Komponente RX (oben links), das zu transferrierende Anion $\mathrm{Y}$ (unten rechts) in Form eines Salzes, das sich in der wässrigen Phase löst, sowie der Phasentransferkatalysator $\mathrm{Q}^{+}$. Die organische Phase kann ein Lösungsmittel enthalten (besonders bei festen organischen Verbindungen RX sinnvoll), kann aber auch bei einem flüssigen Reagenz nur aus der reinen Verbindung bestehen.

Das quartäre Kation steht in der wässrigen Phase in einem Gleichgewicht, in dem es anteilig an das zu transferrierende Anion $\mathrm{Y}^{-}$koordiniert ist. Durch den lipophilen Rest 


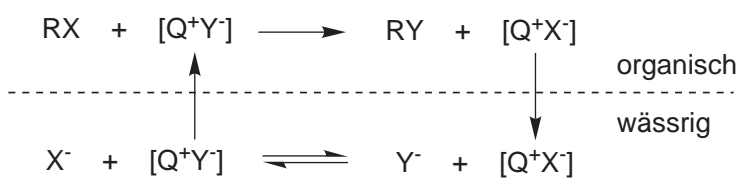

Schema 5.3 Das Grundprinzip der Phasentransferkatalyse. ${ }^{[105-110]} \mathrm{R}=$ organischer Rest, $\mathrm{Q}^{+}=$quartäres Kation des Phasentransferkatalysators, $\mathrm{Y}^{-}=\mathrm{zu}$ transferrierendes Anion, $\mathrm{X}^{-}=$Abgangsgruppe der organischen Verbindung.

ist ein Übergang in die organische Phase möglich, wo es mit dem Edukt RX zu dem gewünschten Produkt RY reagiert. Anschließend wird die freigesetzte Abgangsgruppe $\mathrm{X}^{-}$von $\mathrm{Q}^{+}$in die wässrige Phase transferriert, wo ein Austausch gegen $\mathrm{Y}^{-}$erfolgt. Der Kreislauf schließt sich. Da es sich bei der Reaktion in der wässrigen Phase um eine Gleichgewichtsreaktion handelt, ist es unter Umständen nötig, das Salz, welches $Y^{-}$ freisetzt, im Überschuss einzusetzen, damit die Reaktion vollständig abläuft.

Schema 5.4 zeigt die chemische Struktur von typischen Phasentransferkatalysatoren. Aliquat $336^{\circledR}$ ist der eingetragene Markenname von Trioctylmethylammoniumchlorid, einem flüssigen, hochviskosen Phasentransferkatalysator. Da es sich dabei um ein großtechnisches Produkt handelt, besteht es nicht aus reinen $\mathrm{C}_{8}$-Ketten, sondern einer Mischung aus $C_{8}$ - und $C_{10}$-Ketten, wobei vorwiegend $C_{8}$-Ketten vorkommen. Ebenfalls Verwendung in dieser Arbeit fand Tributylhexadecylphosphoniumbromid (TBHDPB), ein quartäres Phosphoniumsalz, das kristallin ist und somit leichter dosierbar.

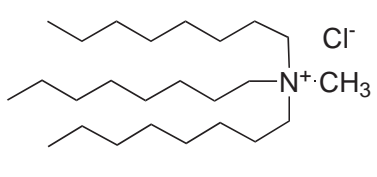

Aliquat 336®

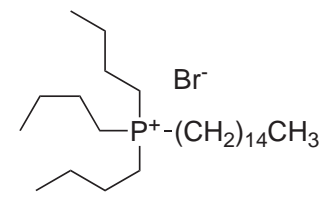

TBHDPB

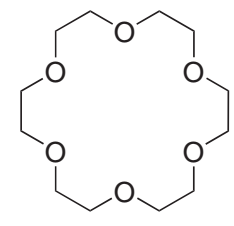

18-Krone-6

Schema 5.4 Die Phasentransferkatalysatoren Trioctylmethylammoniumchlorid (Aliquat $\left.336^{\circledR}\right)$, Tributylhexadecylphosphoniumbromid (TBHDPB) und 1,4,7,10,13,16Hexaoxacyclooctadecan (18-Krone-6).

Eine Alternative $\mathrm{zu}$ den oben vorgestellten quartären Salzen stellen Kronenether dar. ${ }^{[111,112]}$ Dies sind ringförmige Verbindungen, die in regelmäßigen Abständen Sauerstoffatome enthalten. Je nach Ringgröße können somit verschiedene Kationen komplexiert werden. Der abgebildete Kronenether 18-Krone-6 (ein 18-gliedriger Ring mit 6 Sauerstoffatomen) ist aufgrund seines Innendurchmessers für Kalium-Kationen geeignet, kann aber auch für Natrium-Kationen eingesetzt werden. ${ }^{[113]}$ Die Kronenether 
15-Krone-5 und 12-Krone-4 sind ideal für Natrium- bzw. Lithium-Kationen. Das komplexierte Kation kann solvatisiert werden und löst sich aufgrund der lipophilen Außenhülle in der organischen Phase, in die es das Anion transferriert. Je nach Löslichkeit und Lösungsmittel kann eine stöchiometrische oder überstöchiometrische Verwendung des Kronenethers notwendig sein, da er im Gegensatz zu den oben vorgestellten Phasentransferkatalysatoren nicht das Anion komplexiert, sondern das Kation, welches an das Nukleophil gebunden vorliegt.

\subsubsection{Synthese von 4-Vinylbenzylbenzolthiosulfonat (ViBSuT)}

Die Synthese von ViBSuT erfolgte in stark polaren Lösungsmitteln, da sonst das Natriumbenzolthiosulfonat in einer angemessenen Lösungsmittelmenge nicht vollständig gelöst werden konnte. Die Reaktion wurde in wässrigen Systemen unter Zuhilfenahme von Phasentransferkatalysatoren durchgeführt. Aufgrund von niedrigen Produktausbeuten wurde auf Toluol als Lösungsmittel für die organische Phase verzichtet. Die organische Phase bestand somit ausschließlich aus dem Reaktanden 4Vinylbenzylchlorid 22. Neben Wasser wurden Ethanol und 1,2-Dimethoxyethan (DME, Glyme) als polare Lösungsmittel in einer phasentransferkatalysierten Reaktion untersucht.

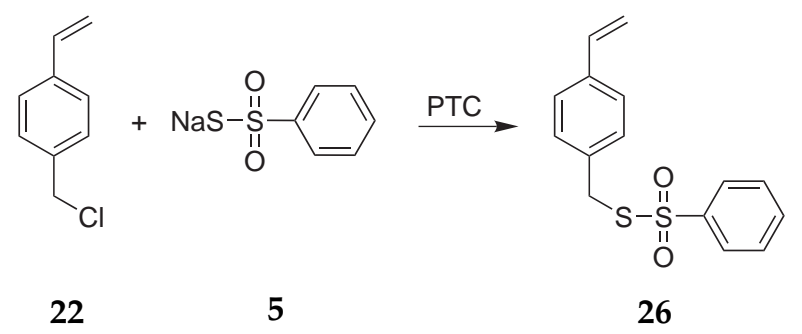

Schema 5.5 Synthese des funktionalisierten Monomers 4-Vinylbenzylbenzolthiosulfonat.

Zusätzlich zu dem gewünschten Produkt wurden zwei Nebenprodukte isoliert und charakterisiert. Dabei handelt es sich um die Hydrolyseprodukte 4Hydroxymethylstyrol 27 und das Dimer Di-(4-Vinylbenzyl)ether 28. Charakteristisch ist die Verschiebung der Methylenprotonen zwischen dem aromatischen System und dem funktionalisierten Rest (siehe Schema 5.6). Darüber hinaus konnten die beiden Nebenprodukte massenspektrometrisch nachgewiesen werden.

Es wurde versucht, die optimalen Reaktionsbedingungen für eine nebenproduktfreie 
Synthese zu finden. Obwohl Eduktreste sowie das Hydrolyseprodukt 27 gut säulenchromatographisch abgetrennt werden können, ist dies bei dem Dimer 28 nicht möglich. Darüber hinaus würde eine quantitative Umsetzung die großtechnische Anwendung erheblich vereinfachen. Tabelle 5.1 listet die unterschiedlichen Ansätze sowie die dazugehörige Produktzusammensetzung auf.

Während durch die Wahl geeigneter Reaktionsbedingungen die vollständige Umsetzung von 22 erreicht werden kann (siehe Tabelle 5.1, A06, A07, A09, A10, A12), ist es nicht möglich, in wässrigem Medium die Bildung eines oder beider Nebenprodukte 27 und $28 \mathrm{zu}$ unterdrücken. Experimente haben gezeigt, dass das Hydrolyseprodukt 27 aus 22 bereits nach $2 \mathrm{~h}$ bei $60^{\circ} \mathrm{C}$ in Wasser gebildet wird (sowohl mit als auch ohne der üblichen Menge PTC). Das Nebenprodukt 28 wird nur in Reaktionen beobachtet, in denen das Natrium-Salz 5 zugegen ist.

Um die Bildung der Nebenprodukte zu unterbinden, wurden verschiedene stark polare organische Lösungsmittel (LM) sowie eine Änderung des pH-Wertes untersucht (Tabelle 5.2). Die besten Ergebnisse wurden dabei mit wasserfreiem Dimethoxyethan (DME; A17, A18) sowie mit N,N-Dimethylformamid (DMF; A23) erzielt. Diese Ansätze waren frei von dem Hydrolyseprodukt 27, enthielten aber dennoch geringe Mengen des Dimers 28 sowie Spuren anderer Verunreinigungen. Eine Aufreinigung mittels Destillation ist nicht möglich und Säulenchromatographie ist aufgrund der Ähnlichkeit der Produkte in großem Maßstab ebenfalls nicht möglich. Die Aufreinigung einer kleinen Rohproduktmenge hat gezeigt, dass es sich bei 26 um einen weißen Feststoff handelt, der somit kristallisiert werden könnte. Praktisch wurde bislang kein geeignetes Lösungsmittelsystem dafür gefunden. Nichtsdestotrotz wurde eine Polymerisation mit dem Rohproduktgemisch A03 durchgeführt, um eine eventuelle Verwendbarkeit zu untersuchen und den Einfluss des Dimers 28 auf eine Polymerisation zu untersuchen. Dies wird in Kapitel 8 vorgestellt. 


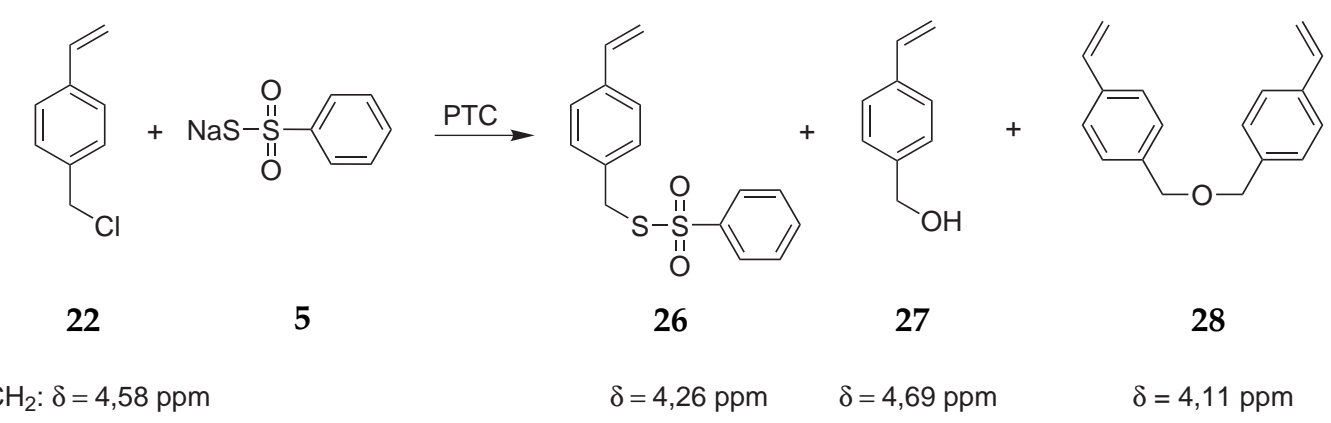

Schema 5.6 Haupt- und Nebenprodukte in der ViBSuT-Synthese. Es sind die Verschiebungen der Methylenprotonen im ${ }^{1} \mathrm{H}-\mathrm{NMR}$-Spektrum (Lösungsmittel: $\mathrm{CDCl}_{3}$ ) angegeben.

Tabelle 5.1 Ergebnisse der Reaktionsoptimierung für die Synthese des funktionalisierten Monomers ViBSuT. Soweit nicht anders angegeben, wurden $5 \mathrm{~mL}$ Wasser auf $1 \mathrm{mmol} 22$ verwendet. ${ }^{a}$ Bezogen auf 1 Äq. 22. ${ }^{b} \mathrm{~A}=$ Aliquat $3366^{\circledR}, \mathrm{B}=$ TBHDPB. ${ }^{c}$ Menge Nebenprodukt in Bezug auf 1 Äq. 26, bestimmt mittels ${ }^{1} \mathrm{H}-\mathrm{NMR} .{ }^{d} 10 \mathrm{~mL}$ Toluol und $7 \mathrm{~mL}$ Wasser auf $1 \mathrm{mmol} 22 .{ }^{e} 6 \mathrm{~mL}$ Toluol und $24 \mathrm{~mL}$ Wasser auf $1 \mathrm{mmol}$ 22. ${ }^{f} 0,4 \mathrm{~mL}$ Toluol und $5 \mathrm{~mL}$ Wasser auf $1 \mathrm{mmol} 22 .{ }^{g}$ Mikrowelle, $P=75 \mathrm{~W} .{ }^{h} 1 \mathrm{~mL}$ Toluol und $5 \mathrm{~mL}$ Wasser auf $1 \mathrm{mmol} 22$.

\begin{tabular}{llclllllll}
\hline$\#$ & Äq. 5 $^{a}$ & PTC $^{b}$ & Äq. PTC & org. LM & T/ ${ }^{\circ} \mathrm{C}$ & $t / \mathbf{h}$ & $\mathbf{2 2}^{c}$ & $\mathbf{2 7}^{c}$ & $\mathbf{2 8}^{c}$ \\
\hline A01 $^{d}$ & 3 & A & 0,035 & Toluol & 110 & 5 & 0,54 & 0,13 & 0,25 \\
A02 $^{e}$ & 1,5 & A & 0,05 & Toluol & 110 & 20 & 0,00 & 0,32 & 0,15 \\
A03 $^{f}$ & 2 & A & 0,05 & Toluol & 110 & 3 & 0,00 & 0,08 & 0,16 \\
A04 $^{g, h}$ & 2 & A & 0,035 & Toluol & 80 & 0,25 & 6,81 & 0,31 & 0,20 \\
A05 $^{g}$ & 2 & A & 0,042 & & 80 & 0,25 & 0,27 & 0,04 & 0,14 \\
A06 & 2 & A & 0,05 & & 110 & 1,5 & 0,00 & 0,17 & 0,11 \\
A07 & 2 & B & 0,05 & & 110 & 1,5 & 0,00 & 0,11 & 0,06 \\
A08 & 1 & B & 0,05 & & 110 & 1,5 & 0,04 & 0,30 & 0,06 \\
A09 & 2 & B & 0,05 & & 110 & 5 & 0,00 & 0,09 & 0,06 \\
A10 & 2 & B & 0,05 & & 110 & 0,5 & 0,00 & 0,07 & 0,16 \\
A11 & 2 & A & 0,05 & & 110 & 0,5 & 0,01 & 0,17 & 0,16 \\
A12 & 2 & A & 0,05 & & 70 & 6 & 0,00 & 0,07 & 0,04 \\
\hline
\end{tabular}


Tabelle 5.2 Ergebnisse der Reaktionsoptimierung für die Synthese des funktionalisierten Monomers ViBSuT. ${ }^{a}$ Bezogen auf 1 Äq. 22. ${ }^{b} \mathrm{~A}=$ Aliquat $336^{\circledR}$, B = TBHDPB. ${ }^{c}$ Konzentration schwankt aufgrund der geringen Einwaagemenge und der hohen Viskosität von A. ${ }^{d} 1 \mathrm{~mL}$ auf $0,1 \mathrm{mmol} 22$. ${ }^{e}$ Menge Nebenprodukt in Bezug auf 1 Äq. 26, bestimmt mittels ${ }^{1} \mathrm{H}-\mathrm{NMR} .{ }^{f}$ Über Natrium getrocknet, Reaktion unter Argon.

\begin{tabular}{lccclccccc}
\hline$\#$ & $\ddot{A q} . \mathbf{5}^{a}$ & PTC $^{b}$ & $\ddot{A q} . \mathrm{PTC}^{a, c}$ & LM $^{d}$ & $T /{ }^{\circ} \mathrm{C}$ & $t / \mathrm{h}$ & $\mathbf{2 2}^{e}$ & $\mathbf{2 7}^{e}$ & $\mathbf{2 8}^{e}$ \\
\hline A13 & 2 & A & 0,10 & Wasser & 60 & 18 & 0,00 & 0,01 & 0,10 \\
A14 & 2 & A & 0,05 & Ethanol & 60 & 18 & 0,00 & 0,08 & 0,20 \\
A15 & 2 & A & 0,10 & DME & 60 & 18 & 0,04 & 0,07 & 0,09 \\
A16 & 1 & & & DME & 60 & 18 & 0,05 & 0,01 & 0,02 \\
A17 & 1 & & & DME & 60 & 6 & 0,05 & 0,00 & 0,03 \\
A18 & 1 & & & DME & 60 & 20 & 0,03 & 0,00 & 0,05 \\
A19 & 2 & A & 0,05 & Essigsäure & 60 & 20 & 0,00 & 0,00 & 0,26 \\
A20 & 2 & A & 0,06 & Puffer pH 5 & 60 & 20 & 0,00 & 0,50 & 0,14 \\
A21 & 2 & A & 0,07 & Puffer pH 7 & 60 & 20 & 0,00 & 0,37 & 0,17 \\
A22 & 2 & B & 0,06 & Puffer pH 7 & 60 & 20 & 0,00 & 0,05 & 0,08 \\
A23 & 1 & & & DMF & 60 & 18 & 0,00 & 0,00 & 0,01 \\
\hline
\end{tabular}




\section{6 RAFT-Polymerisationen}

In diesem Kapitel werden die Polymerisationen vorgestellt, in denen RAFT-Agenzien zum Einsatz gekommen sind. Bei diesen RAFT-Agenzien wurde weiterhin der Ansatz verfolgt, potenziell vulkanisierbare Gruppen zu integrieren, um eine sogenannte $\alpha, \omega$ Funktionalisierung des Polymers zu erreichen. Dadurch können beide Kettenenden des Polymers in das Netzwerk integriert werden und führen so zu einem Material mit verbesserten Eigenschaften. In einem Polymernetzwerk stellen lose Kettenenden, sog. "dangling ends", Defektstellen dar, da sie Spannungen nicht abfangen können und daher nicht zu dem Schermodul beitragen. Nur die Dichte der effektiv elastischen Ketten, also solcher, die sich deformieren können und elastische Energie bei einer NetzwerkDeformation speichern, spielen eine Rolle. ${ }^{[114]}$

Es wurden zwei RAFT-Agenzien erfolgreich synthetisiert, die eine Thioacetat-Funktion enthalten, welche in die R-Gruppe und - in einem Fall - auch in die Z-Gruppe integriert ist. Dabei handelt es sich um ein symmetrisches Trithiocarbonat, dessen Synthese sich gut hochskalieren lässt und es somit auch für eine Anwendung im Technikumsmaßstab zugänglich macht, und ein unsymmetrisches makromolekulares RAFT-Agens (Makro-RAFT-Agens), das in einer mehrstufigen Synthese hergestellt wird und eher für Forschungszwecke geeignet ist. 


\subsection{Trithiocarbonat}

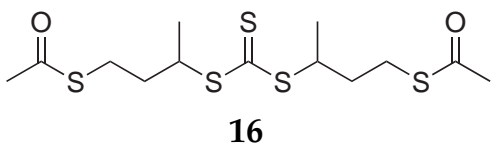

Schema 6.1 Bis(1-methyl-3-thioacetylpropyl)trithiocarbonat

Das symmetrische Trithiocarbonat mit zwei Thioacetat-Funktionen 16 (siehe Schema 6.1) wurde in einer Styrol-Polymerisation eingesetzt. In Anlehnung an die Reaktionsbedingungen, unter denen die Butadien-Polymerisationen durchgeführt werden, wurde in Toluol als Lösungsmittel gearbeitet. Die Polymerisation wurde bei $60^{\circ} \mathrm{C}$ mit AIBN als Initiator durchgeführt und Proben nach 2, 10 und $24 \mathrm{~h}$ entnommen, die mittels GPC analysiert wurden. Die Ergebnisse sind in Tabelle 6.1 und Abbildung 6.1 dargestellt.

Die Polymerisationszeiten wurden mit großen Abständen zueinander gewählt, da so ersichtlicht ist, ob die Molmasse im Laufe der Zeit weiter zunimmt, was jedoch nicht der Fall war. Probe R01A nach $2 \mathrm{~h}$ Polymerisationszeit besteht hauptsächlich aus niedermolekularem Material, bei welchem es sich um molekulares RAFT-Agens handelt. Es wurde eine kleine Menge Polymer (Monomerumsatz 0,04\%) gebildet, dessen mittlere Molmasse und Dispersität in Tabelle 6.1 angegeben sind. Der niedermolekulare Anteil wurde dabei vernachlässigt.

Die Proben R01B und R01C mit 10 bzw. 24 h Polymerisationszeit unterscheiden sich nur unwesentlich. Während in einer RAFT-Polymerisation die mittlere Molmasse mit der Zeit zunimmt, besteht hier nur ein minimaler Unterschied, obwohl die Probe aus der Polymerisation R01C eine mehr als doppelt so lange Polymerisationszeit wie die Probe aus Polymerisation R01B hatte.

Tabelle 6.1 Ergebnisse der Styrol-Polymerisation mit dem RAFT-Agens 16 mit 33 mol-\% Toluol als Lösungsmittel bei $60{ }^{\circ} \mathrm{C}$.

\begin{tabular}{lrccccccc}
\hline$\#$ & $\begin{array}{r}t / \\
\mathrm{h}\end{array}$ & $\begin{array}{c}{[\text { Styrol] }} \\
\mathrm{mol-} \%\end{array}$ & $\begin{array}{c}{[\mathrm{RAFT}] /} \\
\mathrm{mol-} \%\end{array}$ & $\begin{array}{c}{[\mathrm{AIBN}] /} \\
\mathrm{mol}-\%\end{array}$ & $\begin{array}{c}\bar{M}_{\mathrm{n}} / \\
(\mathrm{g} / \mathrm{mol})\end{array}$ & $\begin{array}{c}\bar{M}_{\mathrm{n}}^{\text {theo }} \\
(\mathrm{g} / \mathrm{mol})\end{array}$ & $\begin{array}{c}\mathrm{U} / \\
\%\end{array}$ \\
\hline R01A & 2 & 66,63 & 0,01 & 0,01 & $4,844 \cdot 10^{4}$ & $6,22 \cdot 10^{2}$ & 1,13 & 0,04 \\
R01B & 10 & 66,63 & 0,01 & 0,01 & $2,019 \cdot 10^{5}$ & $2,798 \cdot 10^{4}$ & 1,69 & 5,83 \\
R01C & 24 & 66,63 & 0,01 & 0,01 & $2,152 \cdot 10^{5}$ & $6,682 \cdot 10^{4}$ & 1,70 & 15,77 \\
\hline
\end{tabular}


Insgesamt deutet dies darauf hin, dass eine sekundäre, schwach stabilisierte Abgangsgruppe für Styrol- und somit auch für Butadienpolymerisationen ungeeignet ist. Das Vorgleichgewicht wird nur sehr langsam durchlaufen und es bilden sich kleine Mengen Polymer mit einer vergleichsweise hohen Molmasse in einer freien radikalischen Styrolpolymerisation.

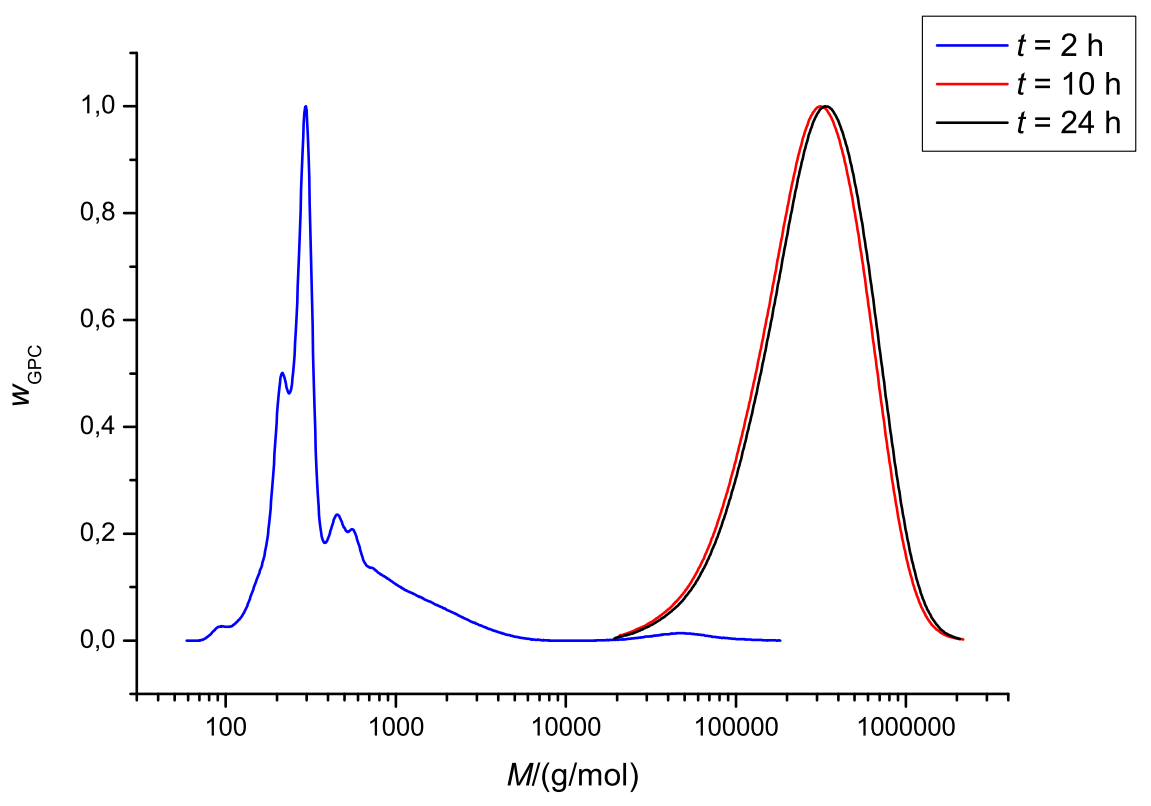

Abbildung 6.1 Molmassenverteilung der Styrol-RAFT-Polymerisationen R01A - C (siehe Tabelle 6.1).

Im Vergleich mit der theoretisch berechneten Molmasse (siehe Abbildung 6.2) zeigt sich, dass die erreichten Molmassen deutlich zu hoch sind. Während das Polymer nach $2 \mathrm{~h}$ Polymerisationszeit nur eingeschränkt beurteilt werden kann, da der Umsatz weit unter einem Prozent liegt und der polymere Anteil in der Molmassenverteilung der GPC nur erahnt werden kann, zeigen auch die beiden Polymere mit längeren Polymerisationszeiten eine Molmasse, die nicht mit dem Umsatz in einer RAFT-Polymerisation korreliert, sondern auf eine freie radikalische Polymerisation hinweist. Grund dafür könnte neben einer ungeeigneten RAFT-Abgangsgruppe auch eine zu große Initiatorkonzentration sein bzw. ein ungünstiges Verhältnis von Initiator zu RAFT-Agens. Aufgrund der großen Menge unverbrauchten RAFT-Agens nach $2 \mathrm{~h}$ Polymerisationszeit in Kombination mit dem geringen Monomerumsatz von lediglich 0,04 \% ist dies aber unwahrscheinlich. 


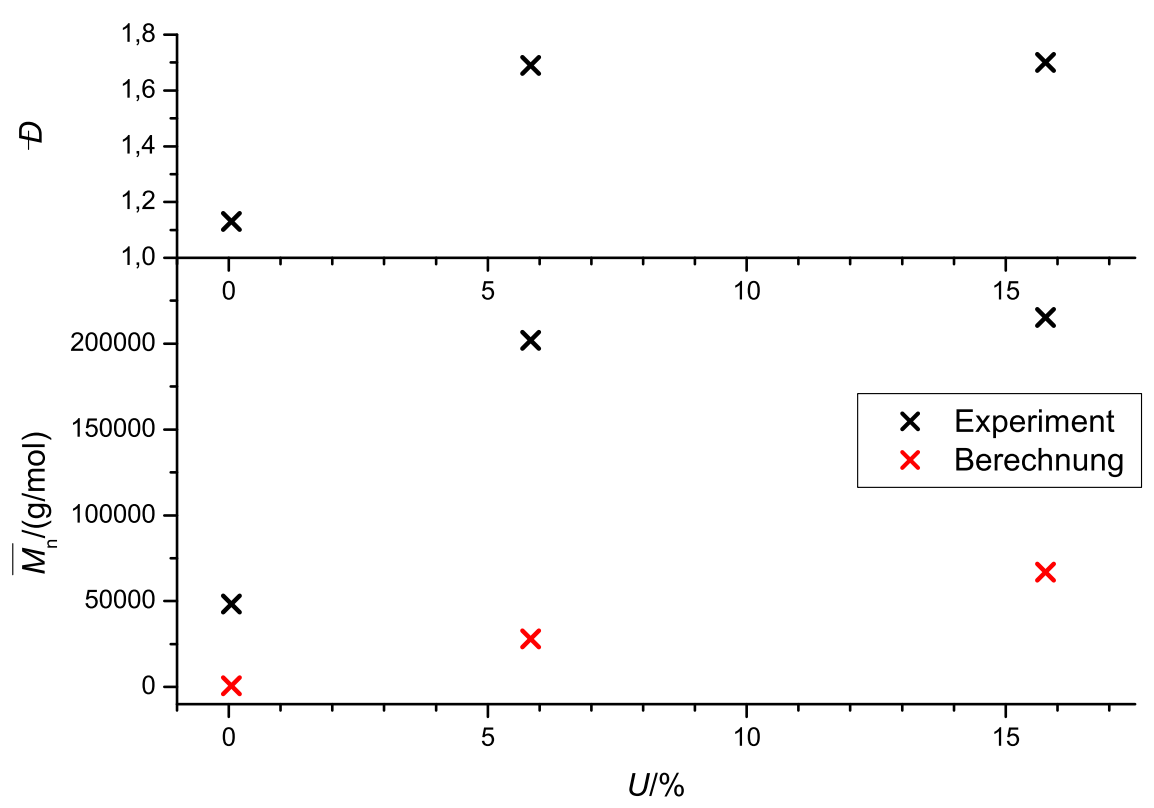

Abbildung 6.2 Darstellung der mittleren Molmassen und der Dispersität in Abhängigkeit vom erreichten Umsatz für die Polymerisationen R01A - C (siehe Tabelle 6.1). 


\subsection{Makro-RAFT-Agens}

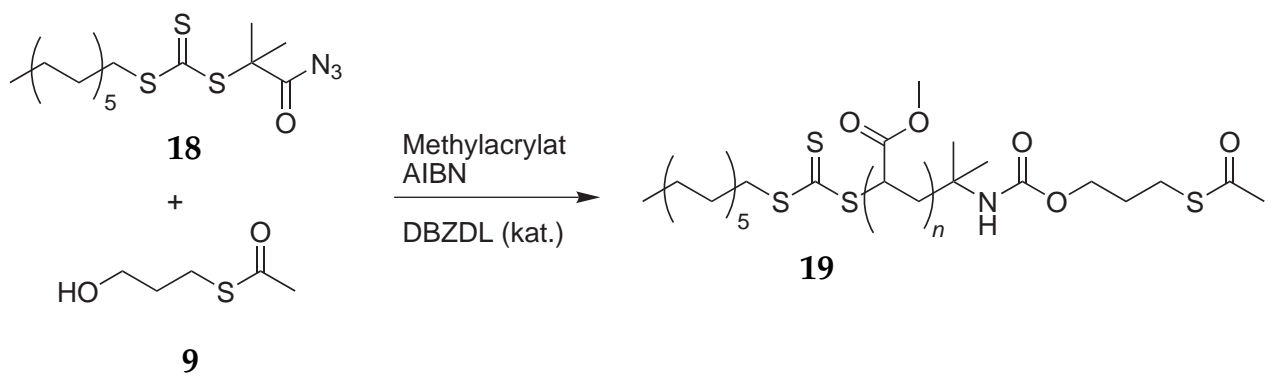

Schema 6.2 Synthese des Makro-RAFT-Agens ${ }^{[99]} 19$.

Das Makro-RAFT-Agens mit der endständigen Azid-Gruppe wurde mit Methylacrylat und AIBN in Anwesenheit des Alkohols 9 und einer katalytischen Menge Dibutylzinndilaurat (DBZDL) polymerisiert, um das funktionalisierte Makro-RAFT-Agens 19 zu erhalten (siehe Schema 6.2). Dieses wurde zur Aufreinigung in Methanol ausgefällt und das Polymer mittels Zentrifugieren und Dekantieren von dem Lösungsmittel abgetrennt und anschließend getrocknet.

Tabelle 6.2 Konzentrationen der Methylacrylat-Polymerisation R02 bei $65^{\circ} \mathrm{C}^{[99]}$ zur Synthese des Makro-RAFT-Agens 19.

\begin{tabular}{cccccccc}
\hline$\#$ & $t /$ & {$[\mathrm{MA}] /$} & {$[\mathrm{RAFT}] /$} & {$[$ Alkohol 9]/ } \\
$\mathrm{h}$ & $\begin{array}{c}\text { mol-\% } \\
\text { mol-\% }\end{array}$ & $\begin{array}{c}\text { mol-\% } \\
\text { mol-\% }\end{array}$ & $\begin{array}{c}\text { mol-\% } \\
\text { mol }\end{array}$ & $\begin{array}{c}\bar{M}_{\mathrm{n}} / \\
(\mathrm{g} / \mathrm{mol})\end{array}$ \\
\hline R02 & 20 & 90,74 & 2,27 & 6,36 & 0,41 & 0,22 & $2,070 \cdot 10^{3}$ \\
\hline
\end{tabular}

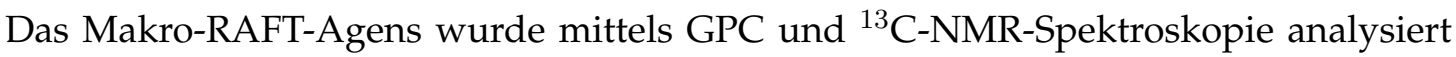
(siehe Abbildung 6.3). Neben dem Kohlenstoffatom der Trithiocarbonat-Gruppe (C1) bei 222,5 ppm ist das Kohlenstoffatom des Thioacetat-Restes (C-2) deutlich sichtbar (194,6 ppm). Das Signal hat sich im Vergleich zum Ausgangsmaterial, dem funktionalisierten Alkohol 9, um 1,0 ppm verschoben (Alkohol: 195,6 ppm). Das Makro-RAFTAgens mit einer mittleren Molmasse von $1797 \mathrm{~g} / \mathrm{mol}$ und einer Dispersität von 1,35 wurde in einer Styrol-Polymerisation eingesetzt, um seine Verwendbarkeit zu überprüfen. Die Konzentrationen und Ergebnisse sind in Tabelle 6.3 aufgelistet. Dabei wurde für die mittlere Molmasse des RAFT-Agens die Styrol-Kalibrierung der GPC mit Hilfe von Mark-Houwink-Koeffizienten an das Methylacrylat-Polymer angepasst. Dies war notwendig, damit eine möglichst korrekte Molmasse des polymeren RAFT-Agens ge- 
messen werden konnte, mit der die Konzentrationen der daran anschließenden StyrolPolymerisation korrekt berechnet werden können. Es bleibt jedoch die Einschränkung, dass es sich hier um ein RAFT-Agens handelt, dessen R- und Z-Gruppen relativ groß sind, und dessen Knäuel- und Diffusionsverhalten sich von dem eines reinen MAPolymers unterscheiden kann. Die mittleren Molmassen der Styrol-Polymere, die in den Polymerisationen R03A - D hergestellt wurden, wurden hingegen mit einer StyrolKalibrierung bestimmt, da hierbei der Styrol-Block überwiegt. Nichtsdestotrotz sind diese mittleren Molmassen keine absoluten Werte, sondern treffen eher eine qualitative Aussage über den Verlauf der Polymerisation.

Tabelle 6.3 Konzentrationen und Ergebnisse der Styrol-Polymerisationen R03A - F mit dem RAFT-Agens R02 bei $60{ }^{\circ} \mathrm{C} .{ }^{a}$ In Methacrylat-Äquivalenten zur Bestimmung der Molmasse des Makro-RAFT-Agens. ${ }^{b}$ In Styrol-Äquivalenten zum besseren Vergleich mit den Polystyrolen R03A-F.

\begin{tabular}{lcccccccc}
\hline$\#$ & $\begin{array}{c}t / \\
\mathrm{h}\end{array}$ & $\begin{array}{c}\text { [Styrol]/ } \\
\text { mol-\% }\end{array}$ & $\begin{array}{c}\text { [RAFT]/ } \\
\text { mol-\% }\end{array}$ & $\begin{array}{c}\text { [AIBN]/ } \\
\text { mol-\% }\end{array}$ & $\begin{array}{c}\bar{M}_{\mathrm{n}} / \\
(\mathrm{g} / \mathrm{mol})\end{array}$ & $\begin{array}{c}\bar{M}_{\mathrm{n}}^{\text {theo }} / \\
(\mathrm{g} / \mathrm{mol})\end{array}$ & $\begin{array}{c}\text { U/ } \\
\%\end{array}$ \\
\hline $\mathrm{R}^{2}{ }^{a}$ & & & & & $1,797 \cdot 10^{3}$ & & 1,35 & \\
$\mathrm{R}^{b}{ }^{b}$ & & & & & $2,070 \cdot 10^{3}$ & & 1,34 & \\
$\mathrm{R} 03 \mathrm{~A}$ & 1 & 99,66 & 0,05 & 0,29 & $3,572 \cdot 10^{3}$ & $3,138 \cdot 10^{3}$ & 1,23 & 3,0 \\
$\mathrm{R} 03 \mathrm{~B}$ & 2 & 99,66 & 0,05 & 0,29 & $4,672 \cdot 10^{3}$ & $3,880 \cdot 10^{3}$ & 1,27 & 5,1 \\
$\mathrm{R} 03 \mathrm{C}$ & 3 & 99,66 & 0,05 & 0,29 & $5,863 \cdot 10^{3}$ & $4,404 \cdot 10^{3}$ & 1,21 & 6,5 \\
$\mathrm{R} 03 \mathrm{D}$ & 4 & 99,66 & 0,05 & 0,29 & $4,716 \cdot 10^{3}$ & $4,497 \cdot 10^{3}$ & 1,43 & 6,8 \\
$\mathrm{R} 03 \mathrm{E}$ & 6 & 99,66 & 0,05 & 0,29 & $5,268 \cdot 10^{3}$ & $4,568 \cdot 10^{3}$ & 1,36 & 7,1 \\
$\mathrm{R} 03 \mathrm{~F}$ & 24 & 99,66 & 0,05 & 0,29 & $5,417 \cdot 10^{3}$ & $6,897 \cdot 10^{3}$ & 1,28 & 14,2 \\
\hline
\end{tabular}

Die Ergebnisse der Polymerisationen R03A - F sind der Tabelle 6.3 zu entnehmen. Es zeigt sich, dass - abgesehen von einer Ausnahme - die mittleren Molmassen mit fortschreitender Polymerisationszeit und zunehmendem Umsatz ansteigen, wobei die Dispersitäten deutlich unter 1,50 bleiben. Die theoretisch berechneten Molmassen spiegeln die im Experiment erreichten Ergebnisse sehr gut wider (siehe Abbildung 6.4). 


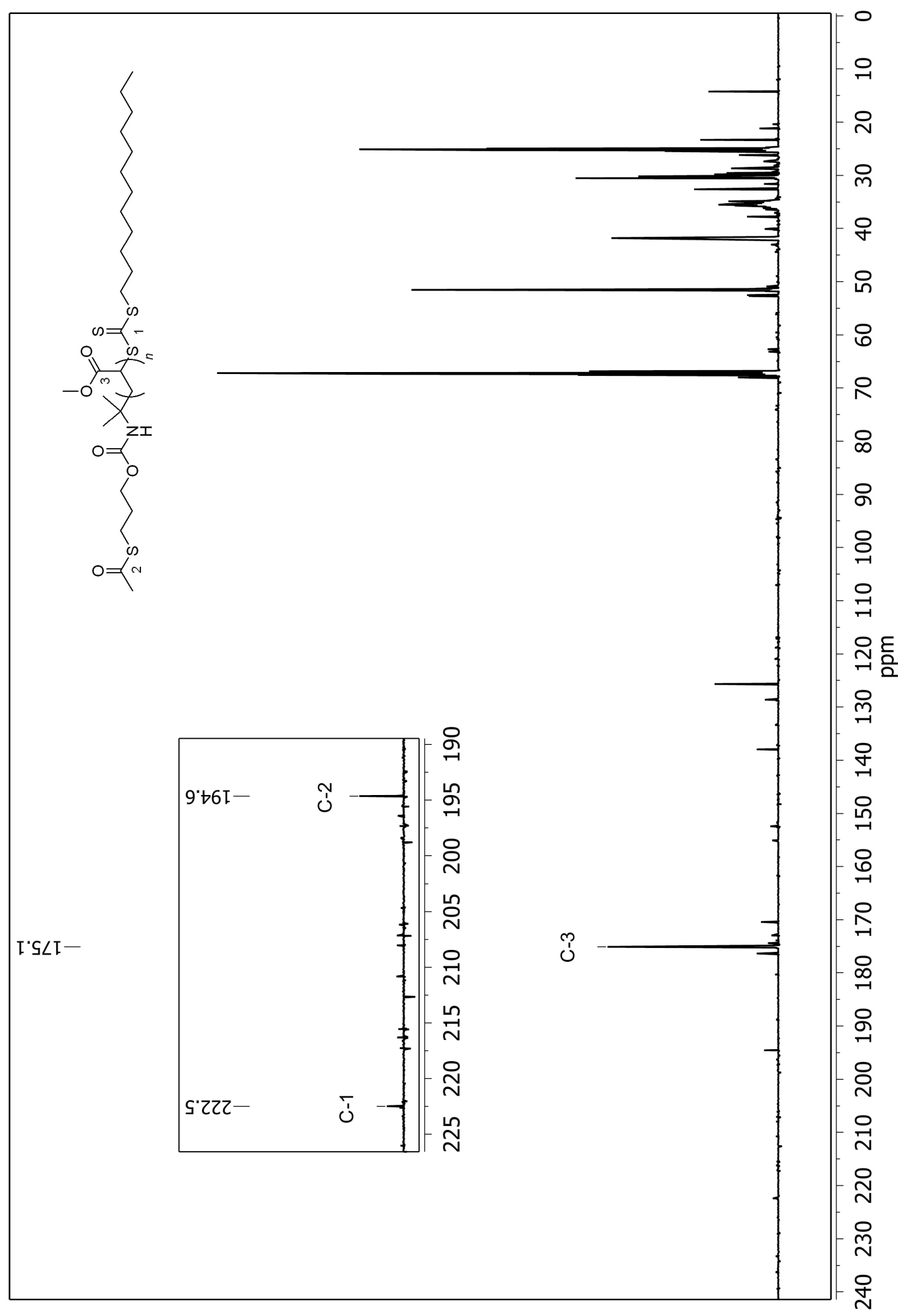

Abbildung 6.3 ${ }^{13} \mathrm{C}-\mathrm{NMR}-S p e k t r u m$ des Makro-RAFT-Agens 19 in THF- $\mathrm{d}_{8}$. Markiert sind die beiden für das RAFT-Agens charakteristischen Kohlenstoffatome C-1 und C-2 sowie das Carbonyl-Kohlenstoffatom des Methylacrylats (C-3). 

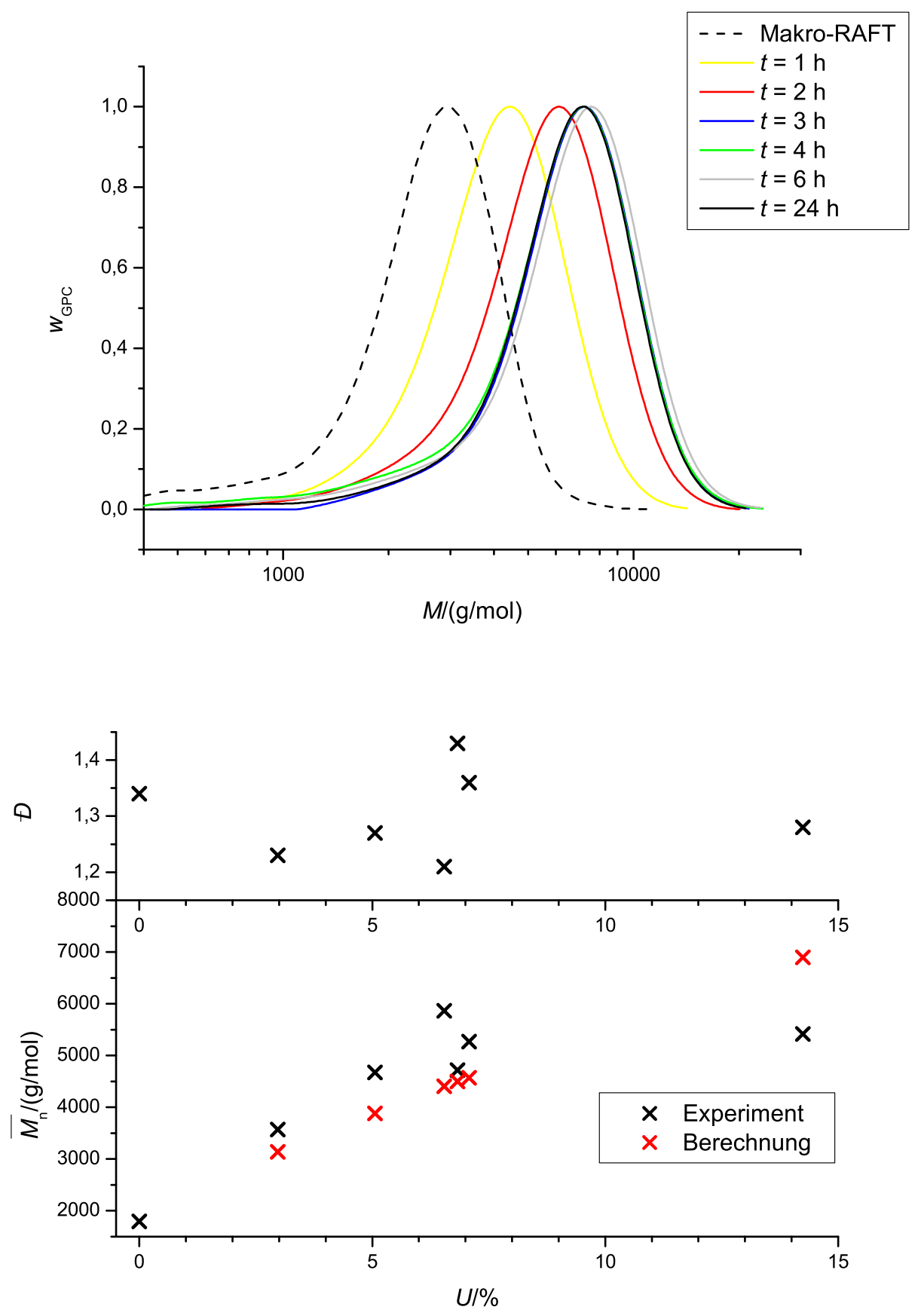

Abbildung 6.4 Molmassenverteilungen (oben) sowie Darstellung der mittleren Molmassen und Dispersitäten in Abhängigkeit vom Umsatz (unten) der StyrolPolymerisationen R03A - F mit dem RAFT-Agens R02 bei $60{ }^{\circ} \mathrm{C}$ (siehe Tabelle 6.3). 


\subsection{Fazit}

Es wurden zwei RAFT-Agenzien erfolgreich synthetisiert und in einer StyrolPolymerisation auf ihre Wirksamkeit hin untersucht. Das bifunktionelle RAFT-Agens 16 konntrollierte das Kettenwachstum nicht, was auf die unzureichend stabilisierte Abgangsgruppe zurückzuführen ist. Nach erfolgter Addition eines Styrol-Makro-Radikals an das RAFT-Agens fragmentiert das Intermediat bevorzugt unter Abspaltung des Styrol-Makro-Radikals anstelle der Abgangsgruppe, wodurch es zur Bildung von Polystyrol ohne RAFT-Gruppe kommt. Der regulierende Effekt, den das RAFT-Agens auf das Kettenwachstum hat, bleibt aus. Um dies zu umgehen, muss die Abgangsgruppe besser stabilisiert werden, was z. B. durch einen Phenyl- oder Estersubstituenten anstelle der Methylgruppe oder einen zusätzlichen Nitrilrest erreicht werden kann. Für diese Art der Abgangsgruppe sind die benötigten Startmaterialien nicht käuflich zu erwerben, sondern müssen erst synthetisiert werden. Dies macht diese Substanzen für den Einsatz in technischen Produkten ungeeignet, da die ohnehin zweistufige Synthese erweitert werden müsste.

Das Makro-RAFT-Agens basiert auf einer außergewöhnlichen RAFT-Agens-Synthese, bei der das gewünschte Produkt erst in einer Polymerisation in situ gebildet wird. Aufgrund seiner kontrollierenden Eigenschaften hat dieses Makro-RAFT-Agens eine niedrige Dispersität und kommt somit einem Agens mit einer definierten Molmasse sehr nahe. Der Methylacrylat-Block ist mit ca. 15 Monomereinheiten sehr kurz und verändert dadurch die Eigenschaften des Polystyrol-Polymers nur unwesentlich. Auf der anderen Seite gewährleistet es eine weitestgehend vollständige Umsetzung des RAFTAgens und damit verbunden die Anbindung des Alkohols. Die erfolgreiche Anbindung des Alkohols konnte mittels NMR-Spektroskopie nachgewiesen werden. In einer anschließenden Styrol-Polymerisation konnte die Eignung des RAFT-Agenzes bestätigt werden. Das Methylacrylat-Makro-Radikal, welches bei der Fragmentierung des Intermediats freigesetzt wird, ist ausreichend stabilisiert und gewährleistet so ein schnelles Durchlaufen des RAFT-Vorgleichgewichts. 



\section{7 \\ Copolymere mit 4-Vinylbenzylthioacetat}

Die Verbindung ViBET findet bislang in sieben Publikationen Erwähnung (laut SciFinder ${ }^{\circledR}$, Stand: September 2013). Darunter befinden sich drei japanische Patente, ${ }^{[115-117]}$ deren Inhalt nicht zugänglich ist. Außerdem eine Veröffentlichung ${ }^{[118]}$ über enzymähnlich aufgebaute Polymere und ein erst kürzlich veröffentlichtes amerikanisches Patent, ${ }^{[119]}$ in dem es um Liganden für Geruchsrezeptoren und olfaktorische Neuronen geht. Des Weiteren ein amerikanisches Patent ${ }^{[104]}$ aus dem Jahr 1960, das die Synthese von ViBET und Derivaten beschreibt sowie kurz auf zwei radikalische Copolymerisationen mit Styrol und Methylmethacrylat eingeht, und ein britisches Patent, ${ }^{[120]}$ welches die ionische Polymerisation mit ViBET thematisiert. Die radikalische Copolymerisation von Butadien mit ViBET ist bislang nicht veröffentlicht. Aus diesen Gründen wurde das Copolymerisationsverhalten von ViBET mit Styrol und Butadien in einer freien radikalischen Polymerisation näher untersucht und außerdem die Synthese überarbeitet, so dass große Mengen quantitativ hergestellt werden können. 


\subsection{Einfluss auf die Copolymerisation}

Da es nur sehr begrenzt Literaturdaten für Styrol-ViBET-Copolymerisationen gibt, wurde zuerst der Einfluss einer geringen Mengen ViBET auf eine Styrol-Polymerisation untersucht. ViBET und Styrol sind sich strukturell sehr ähnlich, so dass eine freie radikalische Polymerisation mit statistisch entlang der Kette eingebautem ViBET erwartet wird. Die mittlere Molmasse sollte sich nicht oder nur geringfügig (aufgrund der höheren Molmasse von ViBET im Vergleich zu Styrol) von einem Polymer aus einer StyrolHomopolymerisation unterscheiden.

Die Polymerisationen wurden in Lösung (Lösungsmittel: Toluol) durchgeführt und die Reaktionsmischung vor der Polymerisation mit Stickstoff gespült, um Sauerstoff, der die Polymerisation inhibieren könnte, zu entfernen. Dieses Vorgehen ist an die Butadienpolymerisationen im Hochdruckautoklaven angelehnt, in dem die Polymerisationen mit Butadien wiederholt werden sollen. Die Konzentrationen sind in mol-\% angegeben, da das Volumen der Monomermischung nicht exakt angegeben werden kann. Die bisweilen relativ große Menge ViBET, die in Styrol (und gegebenenfalls einem Lösungsmittel) gelöst wird, führt zu einer unbekannten Volumenänderung, so dass eine Angabe (z. B. der Radikalkonzentration) in (m)mol/L nicht möglich ist.

Da die Propagationsgeschwindigkeit von Styrol relativ niedrig ist, wurden Polymerisationszeiten von 10 und $24 \mathrm{~h}$ gewählt. Die Zusammensetzungen sind in Tabelle 7.1 aufgeführt, die Ergebnisse in Tabelle 7.2.

Tabelle 7.1 Konzentrationen der Styrol-Polymerisationen M01 - M02. ${ }^{a}$ Referenz für M02.

\begin{tabular}{lccccc}
\hline$\#$ & $\begin{array}{c}{[\text { Styrol]/ }} \\
\text { mol-\% }\end{array}$ & $\begin{array}{c}{[\text { ViBET]/ }} \\
\text { mol-\% }\end{array}$ & $\begin{array}{r}{[\text { AIBN]/ }} \\
\text { mol-\% }\end{array}$ & $\begin{array}{r}{[\text { Toluol]/ }} \\
\text { mol-\% }\end{array}$ & $\begin{array}{c}\text { [ViBET]/[Gesamtmonomer]/ } \\
\text { mol-\% }\end{array}$ \\
\hline M01 $^{a}$ & 66,73 & & 0,01 & 33,36 & \\
M02 & 66,27 & 0,67 & 0,01 & 33,05 & 1 \\
\hline
\end{tabular}

Während der Umsatz sich kaum unterscheidet, gibt es bei der Copolymerisation unerwartete Ergebnisse für die mittleren Molmassen und Dispersitäten. Obwohl nur jedes 100. Monomermolekül eine funktionelle Gruppe in para-Position zu der vinylischen Doppelbindung enthält, scheint dies einen Einfluss auf die Polymerisation zu haben, wie Abbildung 7.1 in der Gegenüberstellung der Molmassenverteilungen der 
vier Polymere zeigt. Während sich die Molmassen und Dispersitäten der Styrolpolymere trotz unterschiedlicher Polymerisationszeiten kaum unterscheiden, nimmt die Molmasse des Copolymers bei längerer Polymerisation deutlich zu. Dennoch haben beide Copolymere sehr viel kürzere Ketten als die Homopolymere zur gleichen Zeit und bei ähnlichem Umsatz.

Tabelle 7.2 Ergebnisse der Styrol-ViBET-Copolymerisation in Toluol bei $60^{\circ} \mathrm{C}$. Die Umsätze beziehen sich auf die Comonomermischung.

\begin{tabular}{lccrr}
\hline$\#$ & $t / \mathrm{h}$ & $\bar{M}_{\mathrm{n}} /(\mathrm{g} / \mathrm{mol})$ & $Ð$ & $U / \%$ \\
\hline M01A & 10 & $1,998 \cdot 10^{5}$ & 1,69 & 7,0 \\
M01B & 24 & $2,144 \cdot 10^{5}$ & 1,71 & 17,0 \\
\hline M02A & 10 & $1,895 \cdot 10^{4}$ & 1,45 & 6,1 \\
M02B & 24 & $3,904 \cdot 10^{4}$ & 1,36 & 15,4 \\
\hline
\end{tabular}

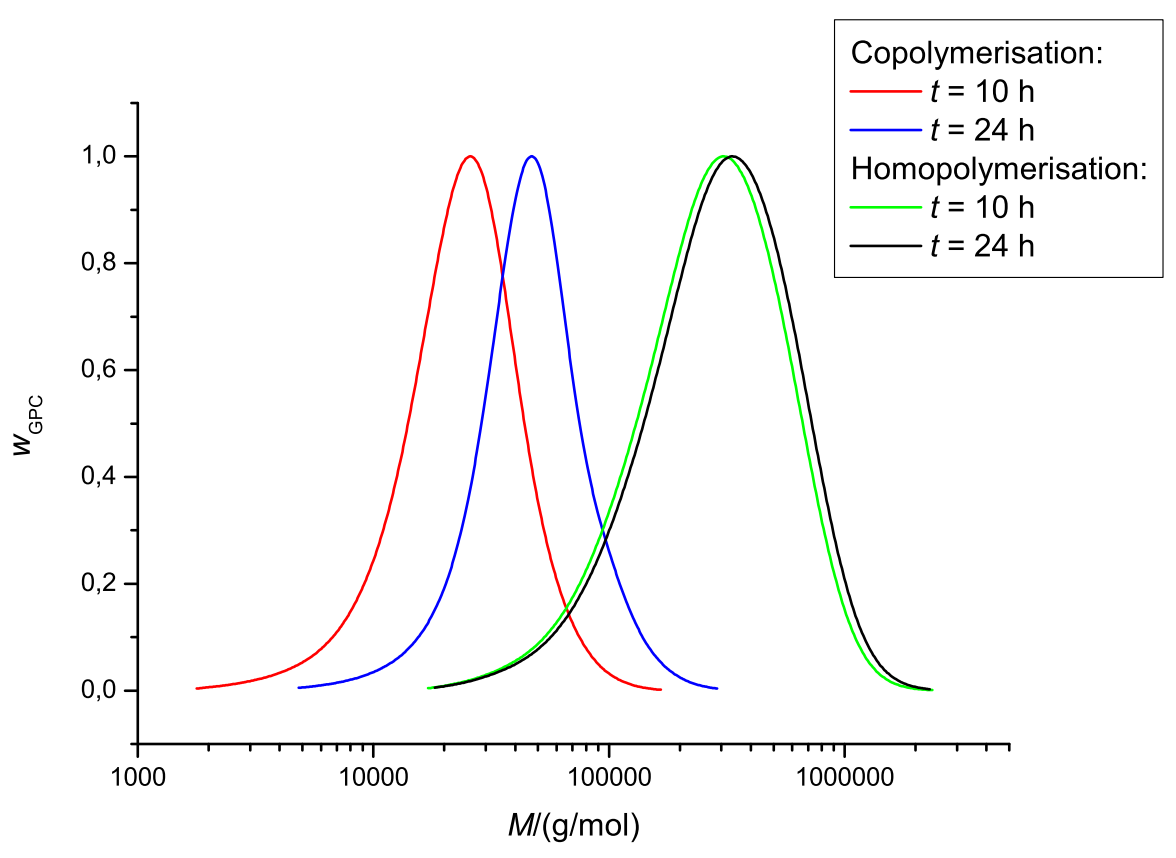

Abbildung 7.1 Molmassenverteilungen der Polymerisationen M01 - M02 (siehe Tabelle 7.1 und Tabelle 7.2). 


\subsection{Konzentrationsabhängiges Verhalten}

Als Konsequenz aus den Ergebnissen der Polymerisationen M01 und M02 wurde der Einfluss der ViBET-Konzentration untersucht, da es ungewöhnlich ist, dass bereits bei einer Konzentration von 1 mol-\% solche Veränderungen auftreten. Weil ein Copolymer mit $2-5$ mol-\% ViBET angestrebt wird, ist der Einfluss größerer Comonomermengen interessant.

Es wurden Polymerisation mit 1, 2 und 3 mol-\% ViBET (bezogen auf Styrol) durchgeführt und kürzere Zeitabstände untersucht $(4,8,16,24 \mathrm{~h})$, weswegen die Referenz und die Polymerisation mit 1 mol-\% wiederholt wurden. Die Ergebnisse der konzentrationsabhängigen Polymerisationen sind in Tabelle 7.4 dargestellt und die dazugehörigen Konzentrationen in Tabelle 7.3.

Tabelle 7.3 Konzentrationen der Styrol-ViBET-Copolymerisation M03 - M06.

${ }^{a}$ Referenz für M04 - M06.

\begin{tabular}{lccccc}
\hline$\#$ & $\begin{array}{r}{[\text { Styrol]/ }} \\
\text { mol-\% }\end{array}$ & $\begin{array}{c}{[\text { ViBET]/ }} \\
\text { mol-\% }\end{array}$ & $\begin{array}{c}{[\text { AIBN]/ }} \\
\text { mol-\% }\end{array}$ & $\begin{array}{c}\text { [Toluol]/ } \\
\text { mol-\% }\end{array}$ & $\begin{array}{c}\text { [ViBET]/[Gesamtmonomer]/ } \\
\text { mol-\% }\end{array}$ \\
\hline M03 $^{a}$ & 66,67 & & 0,01 & 33,32 & \\
M04 & 66,00 & 0,68 & 0,01 & 33,31 & 1 \\
M05 & 65,31 & 1,33 & 0,01 & 33,35 & 2 \\
M06 & 64,60 & 2,01 & 0,01 & 33,38 & 3 \\
\hline
\end{tabular}

Die in Tabelle 7.4 aufgeführten Ergebnisse zeigen deutlich eine Konzentrationsabhängigkeit bei dem Einfluss von ViBET auf die Polymerisationen. Während in einer freien radikalischen Polymerisation Molmassen von über $2 \cdot 10^{5} \mathrm{~g} / \mathrm{mol}$ erreicht wurden, ist die mittlere Molmasse mit zunehmender ViBET-Konzentration in der Monomermischung immer kleiner. Bei 3 mol-\% ViBET sind die Polymerproben nach 4 bzw. $8 \mathrm{~h}$ nicht mehr auswertbar, da der Umsatz nahe null liegt. Lediglich bei einem Zusatz von 1 mol-\% ViBET sind sämtliche Proben auswertbar und lassen sich mit dem Styrolhomopolymer vergleichen. Während die Dispersitäten ähnlich variieren, sind die Umsätze der Copolymerisation geringfügig niedriger. Überraschend ist eine zeit- und umsatzabhängige Zunahme der mittleren Molmasse, die für freie radikalische Polymerisationen untypisch ist und für ein Eingreifen des Comonomers in den Wachstumsschritt spricht. Dies lässt sich bei den Polymerisationen M04 und M05 sehr deutlich beobachten.

Trotz der ungleichen Umsätze wurde für den Vergleich mit den Polystyrolreferenzen 
eine Auftragung gegen den Umsatz gewählt, um den Unterschied in der mittleren Molmasse im Vergleich hervorheben zu können. Abbildung 7.2, oben, zeigt einen deutlichen Unterschied zwischen der freien radikalischen Styrolhomopolymerisation und den drei Copolymerisationen. Neben dem Unterschied in der Größenordnung, in der sich die mittleren Molmassen der Copolymerisationen im Vergleich zur Homopolymerisation bewegen, gibt es einen nahezu linearen Anstieg.

Somit zeigt die Copolymerisation mit ViBET erstaunliche Ähnlichkeit zu einer RAFTPolymerisation: Die Molmasse steigt mit dem Umsatz während die Dispersität deutlich kleiner ist als für Styrolpolymerisationen üblich. Obwohl sie nicht nahe 1,0 liegt, wie bei vielen RAFT-Polymerisationen üblich, sieht man doch einen deutlichen Unterschied (siehe Abbildung 7.1).

Tabelle 7.4 Ergebnisse der Styrol-ViBET-Copolymerisation in Toluol bei $60^{\circ} \mathrm{C}$. Die Umsätze beziehen sich auf die Comonomermischung.

\begin{tabular}{lrcrr}
\hline$\#$ & $t / \mathrm{h}$ & $\bar{M}_{\mathrm{n}} /(\mathrm{g} / \mathrm{mol})$ & $\oplus$ & $U / \%$ \\
\hline M03A & 4 & $2,105 \cdot 10^{5}$ & 1,88 & 2,6 \\
M03B & 8 & $1,724 \cdot 10^{5}$ & 2,22 & 5,5 \\
M03C & 16 & $1,811 \cdot 10^{5}$ & 2,19 & 10,6 \\
M03D & 24 & $1,947 \cdot 10^{5}$ & 1,99 & 14,8 \\
\hline M04A & 4 & $1,934 \cdot 10^{3}$ & 4,96 & 0,3 \\
M04B & 8 & $1,828 \cdot 10^{4}$ & 1,80 & 2,6 \\
M04C & 16 & $3,422 \cdot 10^{4}$ & 2,00 & 6,9 \\
M04D & 24 & $4,798 \cdot 10^{4}$ & 1,83 & 10,4 \\
\hline M05A & 4 & $5,96 \cdot 10^{2}$ & 2,36 & 0,0 \\
M05B & 8 & $2,599 \cdot 10^{3}$ & 2,95 & 1,1 \\
M05C & 16 & $1,482 \cdot 10^{4}$ & 1,56 & 7,0 \\
M05D & 24 & $2,638 \cdot 10^{4}$ & 1,77 & 12,4 \\
\hline M06A & 4 & $6,66 \cdot 10^{2}$ & 9,32 & 0,0 \\
M06B & 8 & $6,15 \cdot 10^{2}$ & 2,94 & 0,0 \\
M06C & 16 & $8,710 \cdot 10^{3}$ & 1,43 & 5,5 \\
M06D & 24 & $8,293 \cdot 10^{3}$ & 1,52 & 3,8 \\
\hline
\end{tabular}

Es bleibt daher die Frage zu klären, warum und wie dieses Monomer Einfluss auf die radikalische Polymerisation nimmt. RAFT-Agenzien werden an dem doppeltgebundenen Schwefelatom von Radikalen angegriffen und fragmentieren unter Abspaltung der 
Gruppe, die zuvor an das verbrückende Schwefelatom gebunden war. Ziel der Synthese war der S-Thioester, bei dem der Schwefel als verbrückendes Atom fungiert, wohingegen beim hier unerwünschten $O$-Thioester der Schwefel doppelt gebunden wäre und der Sauerstoff verbrücken würde. Ob sich das Thioacetatanion während der Synthese umgelagert hat, lässt sich mittels $\operatorname{NMR}\left({ }^{1} \mathrm{H}\right.$ und $\left.{ }^{13} \mathrm{C}\right)$ und Massenspektrometrie überprüfen. Charakteristisch im NMR sind die Verschiebung der $\mathrm{CH}_{2}$-Gruppe in Nachbarschaft zu dem verbrückendem Ester-Atom sowie das Kohlenstoffatom, welches die Doppelbindung zu dem Sauerstoffatom (oder Schwefelatom) ausbildet. In der Massenspektrometrie kann durch harte Ionisierungsmethoden ein Bruch der Esterbindung unter Abspaltung von Acetyl (oder Thioacetyl) herbeigeführt werden. Anhand der Masse des übrig gebliebenen Fragments lässt sich die Struktur ebenfalls verifizieren. Die Analytik (siehe Kapitel 11.6) zeigt, dass bei der Synthese mit dem $S$-Thioester das gewünschte Produkt gebildet worden ist. Eine Umlagerung des $S$-Thioesters während der Polymerisation ist ebenfalls denkbar. Da dies nicht nachgewiesen werden konnte und die Analytik deutlich für einen S-Thioester spricht, wird davon ausgegangen, dass das gewünschte Molekül vorliegt. Um die Lesbarkeit zu erhöhen, wird daher im Folgenden nur von einem Thioester oder Thioacetat gesprochen, es ist jedoch immer ein $S$-Thioester bzw. S-Thioacetat gemeint.

Darüber hinaus besteht die Möglichkeit, dass eine Verunreinigung enthalten ist, die aufgrund ihrer geringen Konzentration nicht mit NMR-Spektroskopie detektiert werden kann. Bei dieser Verunreinigung kann es sich z. B. um das Dithioester-Analogon handeln, welches als RAFT-Agens fungieren kann. Wenn dies ausgeschlossen werden kann, bleibt zu klären, welcher Teil des Moleküls für die „Kontrolle“ hinsichtlich Kettenlänge und Dispersität in der Polymerisation zuständig ist. Dazu können verschiedene Moleküle, die strukturell mit dem ViBET-Molekül verwandt sind, untersucht werden. Dabei bleibt zu klären, ob die Doppelbindung einen Einfluss hat (Ethyl- statt Vinylsubstituent), ob der Schwefel einen Einfluss hat (Sauerstoff statt Schwefel) und ob der aromatische Rest einen Einfluss hat (Ethylrest statt 4-Vinylbenzylrest). 

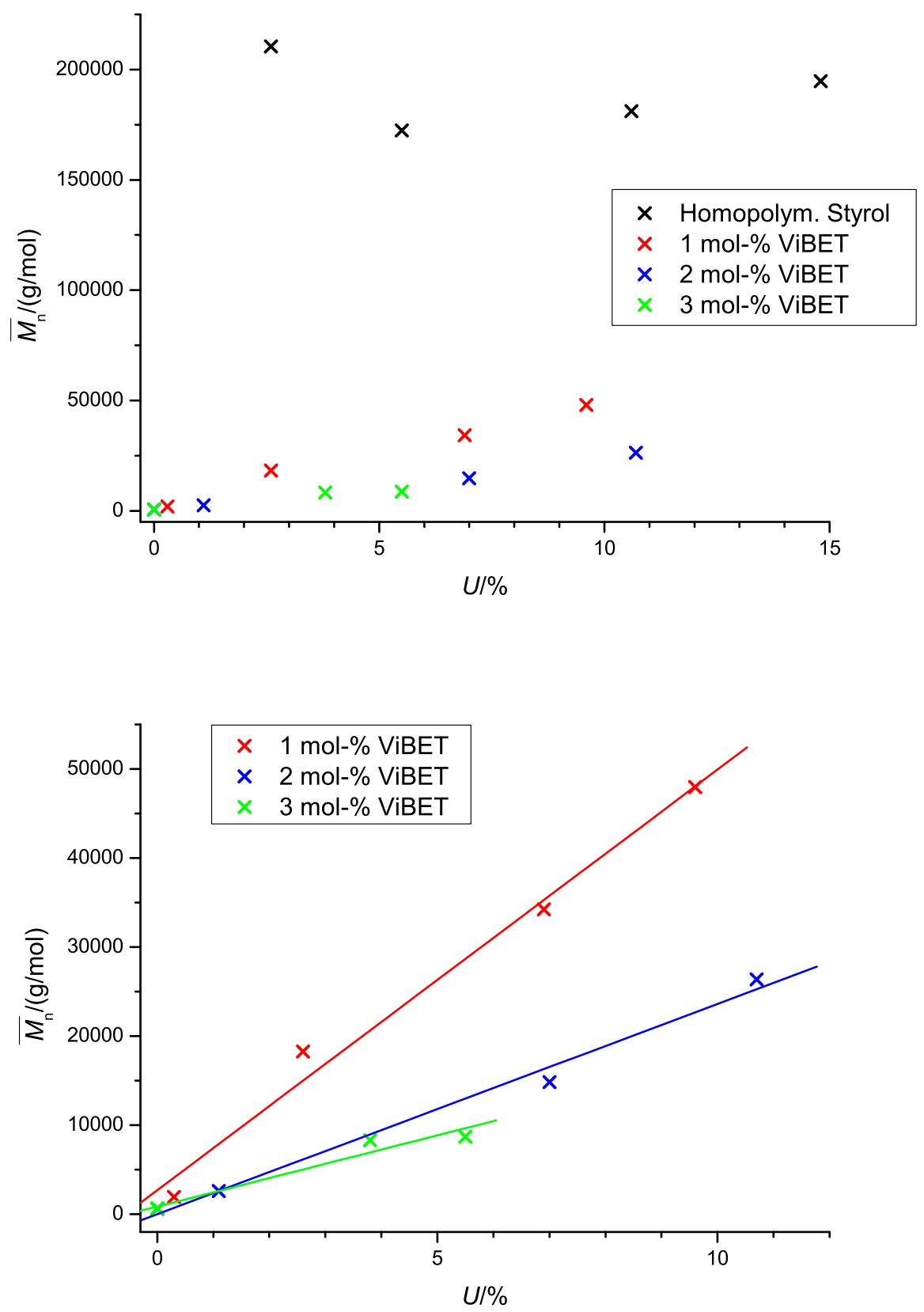

Abbildung 7.2 Darstellung der mittleren Molmassen in Abhängigkeit von der Polymerisationszeit (oben) bzw. dem erreichten Umsatz (unten) für die Polymerisationen M03 - M06 bzw. M04 - M06 (siehe Tabelle 7.3 und Tabelle 7.4). 


\subsection{Polymerisationen mit ViBET-Derivaten}

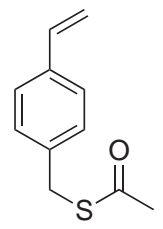

ViBET

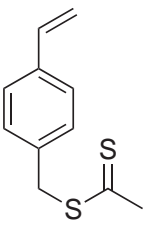

ViBEDiT

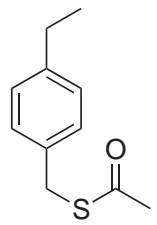

EBET

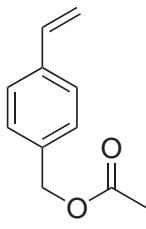

ViBA

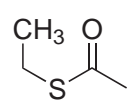

ETA

Schema 7.1 Die verwendeten ViBET-Derivate: 4-Vinylbenzylthioacetat (ViBET), 4-Vinylbenzyldithioacetat (ViBEDiT), 4-Ethylbenzylthioacetat (EBET), 4Vinylbenzylacetat (ViBA) und Ethanthioacetat (ETA).

In Schema 7.1 ist das Monomer ViBET zusammen mit einigen strukturell ähnlichen Verbindungen abgebildet. Variiert wurde die Anzahl der Schwefelatome des Esters von einem (ViBET) zu zweien (ViBEDiT) bzw. gar keinem (ViBA). Darüber hinaus wurde die vinylische Doppelbindung durch einen Ethylrest ersetzt (EBET) sowie der aromatische Rest durch eine Methylgruppe (ETA). Ein Teil dieser Verbindungen konnte käuflich erworben werden (ViBA, ETA), der Rest wurde synthetisiert (ViBEDiT, ViBET, EBET). Die Polymerisationen erfolgten in Substanz, da sie nur zu Forschungszwecken durchgeführt wurden und nicht auf Butadien übertragen werden sollen. Daher wurde auch die Monomermischung entgast. Die Konzentration des Additivs in Bezug auf Styrol liegt bei $1 \mathrm{~mol}-\%$.

Zur Berechnung des Umsatzes wurde die Masse des trockenen Polymers durch die Masse der Monomermischung dividiert. Der Initiator ist zum Zeitpunkt der Wägung größtenteils zerfallen und selbst unzerfallene Anteile würden den Umsatz nicht verfälschen, da weniger als $1 \mathrm{mg}$ auf eine Probe entfällt. Da unklar ist, ob die Additive einpolymerisiert wurden oder schwer flüchtig sind (und somit in dem Polymer verbleiben) oder ob sie abdampfen (und somit nur Polystyrol zurückbleibt), wurden die Umsätze nicht korrigiert.

In Tabelle 7.6 sind die Ergebnisse der Polymerisationen M07 - M12 (siehe Tabelle 7.5) aufgeführt. Eine Probe konnte nicht ausgewertet werden, da sich nach $3 \mathrm{~h}$ Polymerisationszeit noch kein Polymer gebildet hatte (M10A). Die übrigen Proben konnten mittels GPC analysiert und ausgewertet werden.

Die Dispersitäten schwanken größtenteils zwischen 1,50 und 1,72. Es gibt Ausreißer nach oben (M10C und M10D mit 1,85 bzw. 1,80) sowie nach unten (M08C und M08D mit 1,29 bzw. 1,26). Bei ViBET als Additiv wurde dies auch bei den vorherigen Poly- 
Tabelle 7.5 Konzentrationen der Styrol-Polymerisation M07 - M12. ${ }^{a}$ Referenz für M08 - 12.

\begin{tabular}{lcccc}
\hline$\#$ & $\begin{array}{r}\text { [Styrol]/ } \\
\text { mol-\% }\end{array}$ & Additiv & $\begin{array}{c}\text { [Additiv]/ } \\
\text { mol-\% }\end{array}$ & $\begin{array}{c}\text { [AIBN]/ } \\
\text { mol-\% }\end{array}$ \\
\hline M07 $^{a}$ & 99,99 & & & 0,01 \\
M08 & 98,99 & ViBET & 1,00 & 0,01 \\
M09 & 98,92 & ViBEDiT & 1,07 & 0,01 \\
M10 & 98,99 & EBET & 1,00 & 0,01 \\
M11 & 98,99 & ViBA & 1,00 & 0,01 \\
M12 & 98,99 & ETA & 1,00 & 0,01 \\
\hline
\end{tabular}

merisationen beobachtet, allerdings konnte dieser Effekt nicht mit einem der anderen Additive reproduziert werden. Die mittleren Molmassen schwanken stark und unterscheiden sich um mehrere Größenordnungen. Während die Polymerisationen mit ViBA und ETA als Additiv ähnlich große Molmassen liefern wie die Styrol-Polymerisation ohne Additive (im Bereich von $10^{5} \mathrm{~g} / \mathrm{mol}$ ), scheint die Zugabe von EBET oder ViBEDiT eine vergleichbare Auswirkung zu haben wie die Zugabe von ViBET: Die mittlere Molmasse sinkt, die Ketten werden kürzer. Die Polymerisation M10 in Anwesenheit von EBET ist die einzige Polymerisation, bei der der Umsatz deutlich herabgesetzt ist und nach $24 \mathrm{~h}$ nicht im zweistelligen Bereich liegt.

Abbildung 7.3 zeigt die mittleren Molmassen in Abhängigkeit vom Monomerumsatz. Es sind deutlich drei Gruppen zu erkennen. Die Polymerisation mit Styrol ohne weitere Additive (abgesehen vom Initiator AIBN) ist die Referenz und zeigt den Verlauf einer freien radikalischen Polymerisation. ViBA als Additiv in einer Styrol-Polymerisation beeinflusst weder die mittlere Molmasse, noch die Dispersität oder den Umsatz. Es gibt eine kleine Abweichung bei den Polymeren nach $24 \mathrm{~h}$ Polymerisationszeit hinsichtlich mittlerer Molmasse und Umsatz, wobei es sich aber um einen statistischen Fehler handeln kann. Da es sich bei ViBA um das schwefelfreie ViBET-Analogon handelt, zeigt sich deutlich, dass der fehlende Schwefel die Änderung bewirkt.

ETA, das Derivat mit der Methylgruppe anstelle des Styrolrestes, hat einen geringen Einfluss. Die mittleren Molmassen sind um rund $10^{5} \mathrm{~g} / \mathrm{mol}$ kleiner als die Referenzpolymere, wobei die Tendenz mit zunehmender Polymerisationszeit abnimmt. Dieses Molekül enthält einen Schwefelester, genau wie ViBET. Eine Vergrößerung des Ausschnittes, der die höhermolekularen Polymere zeigt, ist in Abbildung 7.4 dargestellt. 
Tabelle 7.6 Ergebnisse der Styrol-Polymerisationen bei $60^{\circ} \mathrm{C}$. Die Umsätze beziehen sich auf die Comonomermischung. ${ }^{a}$ Umsatz zu gering, nicht auswertbar.

\begin{tabular}{|c|c|c|c|c|c|}
\hline \# & Additiv & $t / h$ & $\bar{M}_{\mathrm{n}} /(\mathrm{g} / \mathrm{mol})$ & 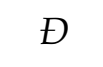 & $U / \%$ \\
\hline M07A & & 3 & $3,588 \cdot 10^{5}$ & 1,59 & 3,0 \\
\hline M07B & & 6 & $3,903 \cdot 10^{5}$ & 1,57 & 5,6 \\
\hline M07C & & 12 & $3,679 \cdot 10^{5}$ & 1,61 & 10,3 \\
\hline M07D & & 24 & $3,951 \cdot 10^{5}$ & 1,62 & 18,8 \\
\hline M08A & ViBET & 3 & $1,565 \cdot 10^{4}$ & 1,61 & 2,8 \\
\hline M08B & ViBET & 6 & $2,135 \cdot 10^{4}$ & 1,52 & 4,6 \\
\hline M08C & ViBET & 12 & $3,669 \cdot 10^{4}$ & 1,29 & 7,9 \\
\hline M08D & ViBET & 24 & $5,806 \cdot 10^{4}$ & 1,26 & 13,4 \\
\hline M09A & ViBEDiT & 3 & $5,25 \cdot 10^{2}$ & 1,60 & 3,3 \\
\hline M09B & ViBEDiT & 6 & $5,96 \cdot 10^{2}$ & 1,55 & 4,8 \\
\hline M09C & ViBEDiT & 12 & $7,84 \cdot 10^{2}$ & 1,59 & 8,1 \\
\hline M09D & ViBEDiT & 24 & $1,245 \cdot 10^{3}$ & 1,62 & 14,6 \\
\hline${\mathrm{M} 10 \mathrm{~A}^{a}}^{a}$ & EBET & 3 & & & \\
\hline M10B & EBET & 6 & $1,111 \cdot 10^{4}$ & 1,59 & 1,4 \\
\hline M10C & EBET & 12 & $1,693 \cdot 10^{4}$ & 1,85 & 2,2 \\
\hline M10D & EBET & 24 & $3,108 \cdot 10^{4}$ & 1,80 & 3,6 \\
\hline M11A & ViBA & 3 & $3,573 \cdot 10^{5}$ & 1,60 & 2,9 \\
\hline M11B & ViBA & 6 & $3,833 \cdot 10^{5}$ & 1,56 & 5,5 \\
\hline M11C & ViBA & 12 & $3,669 \cdot 10^{5}$ & 1,63 & 10,6 \\
\hline M11D & ViBA & 24 & $3,476 \cdot 10^{5}$ & 1,69 & 17,5 \\
\hline M12A & ETA & 3 & $2,469 \cdot 10^{5}$ & 1,65 & 2,6 \\
\hline M12B & ETA & 6 & $2,448 \cdot 10^{5}$ & 1,68 & 4,8 \\
\hline M12C & ETA & 12 & $2,683 \cdot 10^{5}$ & 1,70 & 9,0 \\
\hline M12D & ETA & 24 & $3,145 \cdot 10^{5}$ & 1,72 & 16,3 \\
\hline
\end{tabular}




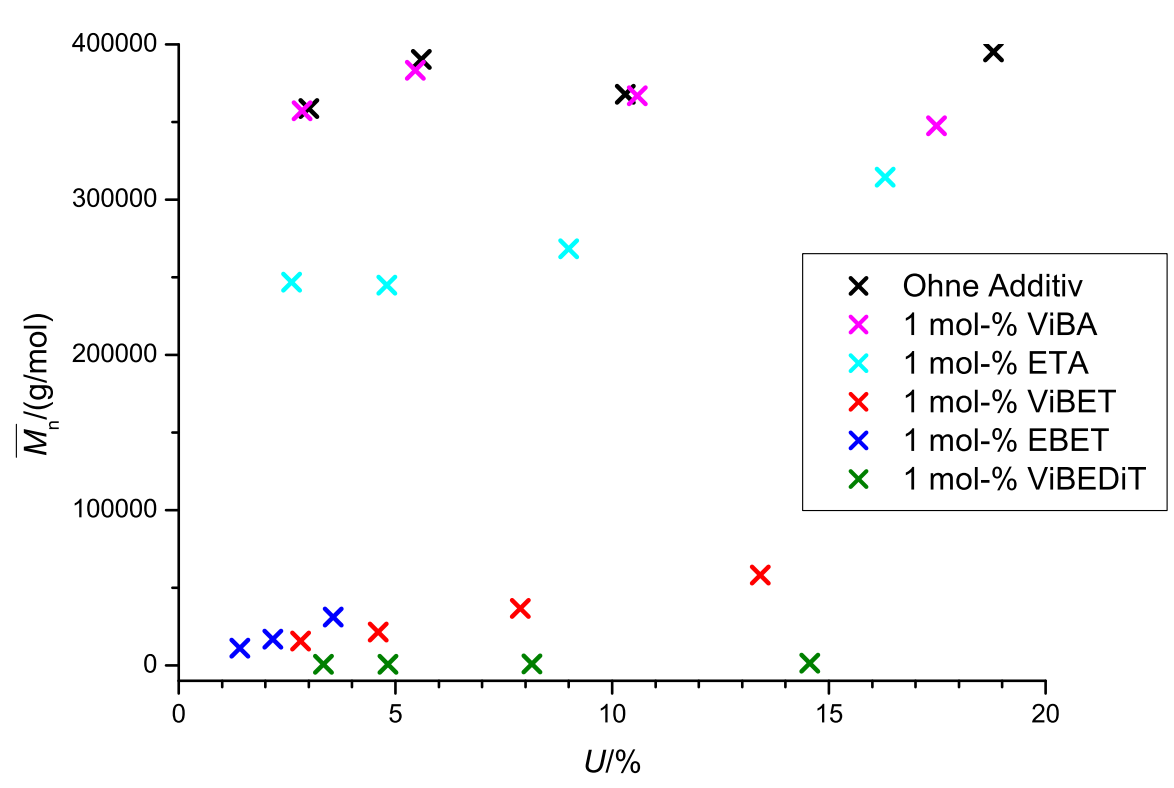

Abbildung 7.3 Darstellung der mittleren Molmassen in Abhängigkeit vom erreichten Umsatz für die Polymerisationen M07 - M12 (siehe Tabelle 7.5 und Tabelle 7.6).

Interessant ist besonders die Vergrößerung des unteren Bereichs, in dem die Polymere mit mittleren Molmassen bis zu $60000 \mathrm{~g} / \mathrm{mol}$ eingezeichnet sind (Abbildung 7.5). Neben ViBET, bei dem bekannt ist, dass es die Bildung kurzer Ketten fördert, finden sich dort die Polymere aus Polymerisationen mit EBET und ViBEDiT als Additiv. Dabei handelt es sich um die Verbindung mit einem gesättigten Rest am Phenylring, die nicht als Monomer fungieren kann (EBET), sowie den Dithioester ViBEDiT. Beide scheinen einen Einfluss auf die Polymerisation auszuüben, der allerding stark unterschiedlich ist. Die Polymerisation mit 1 mol-\% EBET zeichnet sich durch besonders geringe Umsätze aus. Nach $3 \mathrm{~h}$ ist die Polymerisation noch nicht weit genug fortgeschritten, um das Polymer in der GPC analysieren zu können, da die Intensität des Polymers im Vergleich zu den niedermolekularen Rückständen viel zu gering ist. Die übrigen drei Polymerproben zeigen mittlere Molmassen bis zu rund $30000 \mathrm{~g} / \mathrm{mol}$ und damit verbunden einen relativ starken Anstieg der Molmasse bei geringer Zunahme des Umsatzes. Die Polymerisation mit einer äquimolaren Menge ViBEDiT zeigt ein gegenläufiges Verhalten: Die Umsätze steigen stark an und erreichen Werte, die vergleichbar sind mit denjenigen aus der Polymerisation mit ViBET als Additiv. Die mittleren Molmassen sind allerdings sehr gering und liegen selbst nach $24 \mathrm{~h}$ kaum über $1000 \mathrm{~g} / \mathrm{mol}$ und somit zwei Potenzen unter den mittleren Molmassen der Styrol-Referenzpolymerisation. 
Ein Vergleich dieser Polymerisationen zeigt, dass sowohl der Schwefel im Thioester einen Einfluss hat als auch das aromatische System. Der Thioester alleine bewirkt kürzere Polymerketten (siehe ETA, M12A-D), aber der Einfluss wird durch ein aromatisches System noch weiter verstärkt (siehe ViBET, M08A-D, und EBET, M10A-D). Der Dithioester stellt eine Besonderheit dar, da hier der Umsatz trotz sehr kleiner mittlerer Molmassen stark ansteigt. Dies deutet darauf hin, dass übermäßig viele Übertragungsreaktionen stattfinden, die zur Bildung von Oligomeren führen. Dies ist der genaue Gegensatz zu den Polymeren M10A - D, die einen geringen Umsatz bei höheren mittleren Molmassen aufweisen und somit im Verhältnis weniger, aber deutlich längere Polystyrolketten bilden.

In Abbildung 7.5 wurden darüber hinaus die theoretisch berechneten mittleren Molmassen für den Fall, dass ViBET sich wie ein RAFT-Agens verhält, eingezeichnet. Zur Berechnung wurden die Umsätze der Polymerisationen M08A-D verwendet. Es ist deutlich zu erkennen, dass die im Experiment erreichten Molmassen für ein RAFTähnliches Verhalten von ViBET deutlich zu hoch sind und dieses somit ausgeschlossen werden kann. ViBEDiT hingegen, das Dithioester-Äquivalent, gibt die theoretisch berechneten Werte gut wieder.

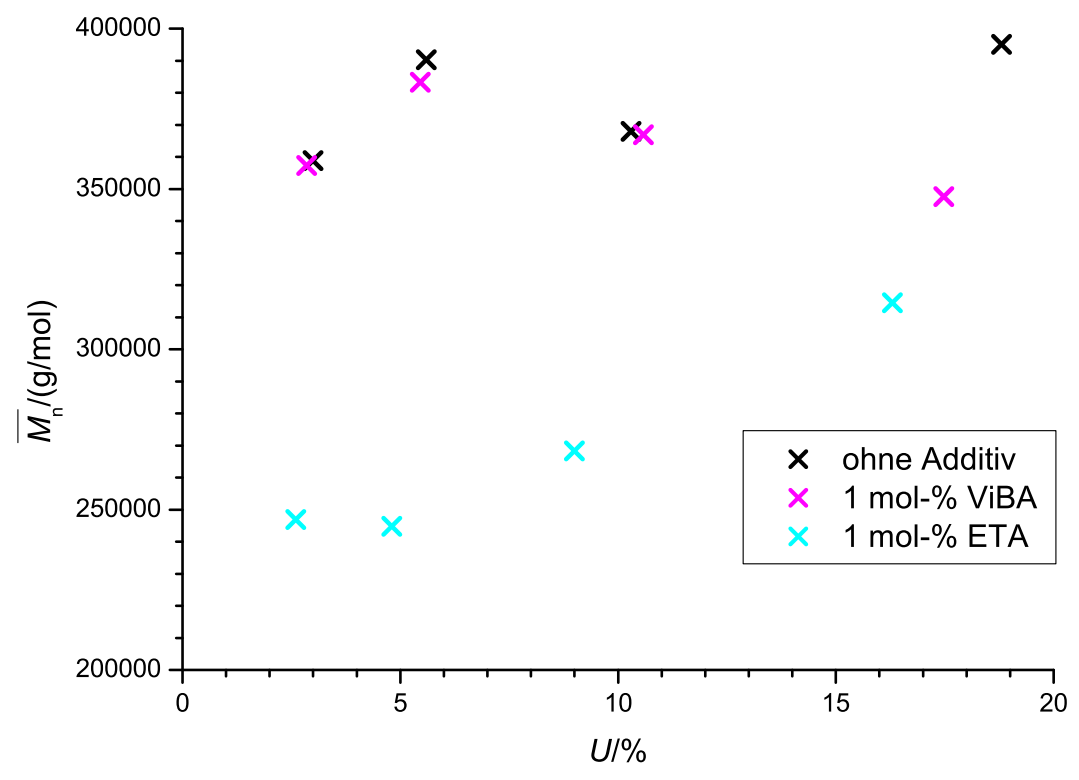

\footnotetext{
Abbildung 7.4 Darstellung der mittleren Molmassen in Abhängigkeit vom erreichten Umsatz für die Polymerisationen M07, M11 und M12 (siehe Tabelle 7.5 und Tabelle 7.6).
} 


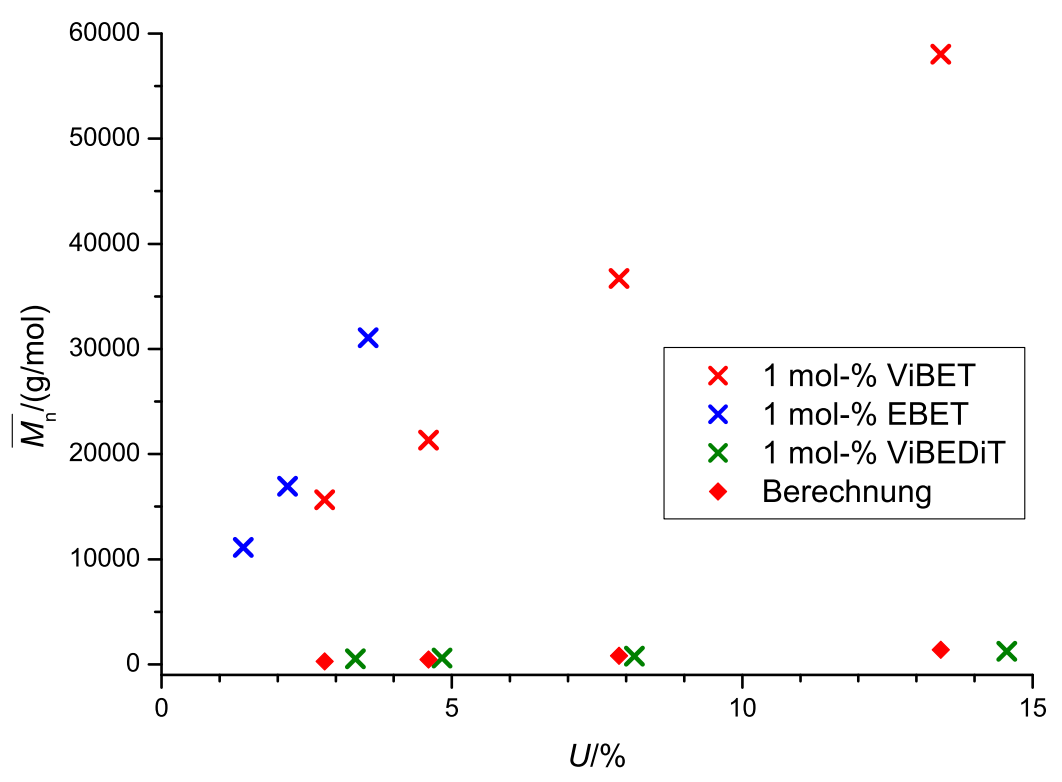

Abbildung 7.5 Darstellung der mittleren Molmassen in Abhängigkeit vom erreichten Umsatz für die Polymerisationen M08 - M10 (siehe Tabelle 7.5 und Tabelle 7.6) sowie die theoretisch berechneten Molmassen nach Formel 2.40. 


\subsection{ESR-Spektroskopie}

Um eine mögliche Verunreinigung, die in die radikalische Polymerisation eingreift, $\mathrm{zu}$ charakterisieren, wurde ESR-Spektroskopie verwendet $(\mathrm{ESR}=$ Elektronenspinresonanz). Im sog. Continuous Wave Verfahren (engl. für kontinuierliche Wellenlänge) werden ungepaarte Elektronen durch Mikrowellenstrahlung konstanter Frequenz angeregt und die Absorption der elektromagnetischen Strahlung in einem sich verändernden Magnetfeld gemessen, in dem sonst entartete Energieniveaus aufspalten (ZeemanEffekt).

In Abhängigkeit von den Atomen, in deren Nähe sich das ungepaarte Elektron befindet, ergibt sich ein Aufspaltungsmuster, das Rückschlüsse auf die Radikalstruktur erlaubt. Es wurden verschiedene Spektren sowohl in Lösung, als auch in Substanz, aufgenommen. Die beste Auflösung hat das Spektrum, das in Abbildung 7.6 dargestellt ist und bei $40^{\circ} \mathrm{C}$ mit Initiator-Zugabe gemessen wurde (Messparameter: siehe Kapitel 11.5). Dieses Radikal, das sich schon bei relativ niedrigen Temperaturen bildet und äußerst stabil ist, gehört nicht zu einem ungepaarten Elektron des ViBET-Moleküls oder des Initiators, sondern zu einem Inhibitor. 3,5-Di-tert-butyl-4-hydroxytoluol („Butylhydroxytoluol“" BHT) wird als Inhibitor dem Lösungsmittel THF sowie Monomeren als Radikalfänger zugesetzt. Bei THF wird so die Bildung hochexplosiver Peroxide unterdrückt und bei Monomeren die ungewollte Polymerisation während der Lagerung.

Wie dieser Inhibitor in die Substanz gelangt, kann nicht sicher gesagt werden. Die Menge ist zu gering, um den Inhibitor mittels NMR-Spektroskopie oder anderer Analytik sehen zu können, weshalb er nicht vorher entdeckt wurde. Nachdem die Synthese in THF erfolgte, ist es möglich, dass er sich aus dem Lösungsmittel in der Substanz abgelagert hat. Eine weitere potenzielle Quelle ist das Monomer 4-Vinylbenzylchlorid. Keiner der Hersteller, von denen die Substanz bezogen wurde (Fluka und Sigma-Aldrich), hat BHT als Inhibitor angegeben, dennoch kann es sich um eine mögliche Quelle handeln. Eine Destillation der verwendeten Substanzen reicht nicht aus, da der Inhibitor sublimiert und somit scheinbar aufgereinigte Fraktionen kontaminiert. Dennoch konnte er nicht bei Trocknung des Monomers im Hochvakuum entfernt werden. Um diese Verunreinigung vollständig zu unterbinden, wurde das Lösungsmittel für die ViBETSynthese gewechselt (Aceton statt THF) und die Reaktionsbedingungen überarbeitet, um weiterhin eine quantitative Umsetzung zu gewährleisten. Darüber hinaus wurde der Einfluss von BHT auf eine freie radikalische Styrol-Polymerisation untersucht. 


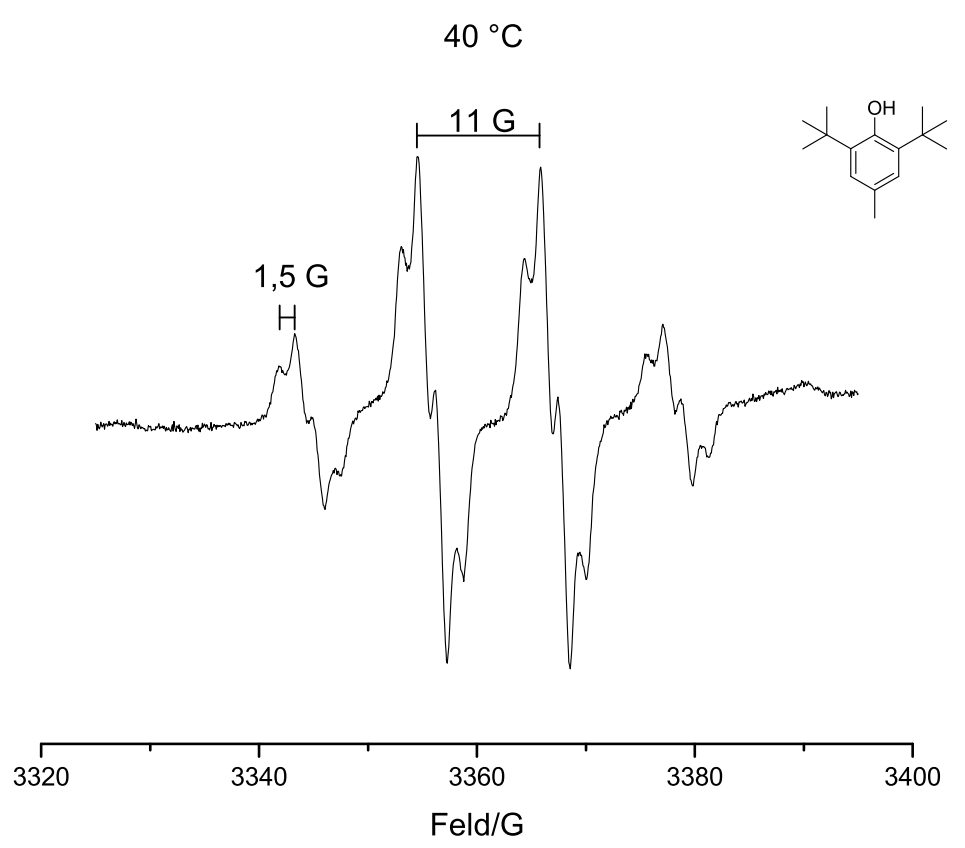

Abbildung 7.6 ESR-Spektrum von ViBET, verunreinigt mit BHT, mit $3 \mathrm{mmol} / \mathrm{L}$ AIBN, gemessen bei $40^{\circ} \mathrm{C}$.

\subsection{Styrol-Polymerisation mit Inhibitor}

Es wurden zwei Styrol-Polymerisationen in Anwesenheit kleiner Mengen BHT durchgeführt. Diese können aufgrund der gewählten Initiator-Konzentration direkt mit den Ergebnissen der Polymerisation M07 verglichen werden.

Tabelle 7.7 Konzentrationen der Styrol-Polymerisation M07, M13 und M14.

${ }^{a}$ Referenz für M13 und M14.

\begin{tabular}{lcccc}
\hline$\#$ & $\begin{array}{c}\text { [Styrol]/ } \\
\text { mol-\% }\end{array}$ & Additiv & $\begin{array}{c}\text { [Additiv]/ } \\
\text { mol-\% }\end{array}$ & $\begin{array}{c}\text { [AIBN]/ } \\
\text { mol-\% }\end{array}$ \\
\hline M07 $^{a}$ & 99,990 & & & 0,010 \\
M13 & 99,983 & BHT & 0,007 & 0,010 \\
M14 & 99,973 & BHT & 0,007 & 0,020 \\
\hline
\end{tabular}

Die Initiator-Konzentration wurde variiert, um den eventuellen Einfluss einer erhöhten Initiator-Konzentration auf BHT in einer Styrol-Polymerisation zu untersuchen. Das Zeitintervall zwischen der Probenentnahme wurde verkürzt, um Veränderungen bei 
der mittleren Molmasse und der Dispersität besser erkennen zu können. Die Konzentrationen sind in Tabelle 7.7 aufgeführt und die Ergebnisse in Tabelle 7.8. Die Polymerisationen M07 und M13 unterscheiden sich lediglich in dem BHT-Zusatz, der M07 fehlt. Ein Vergleich der Polymere, die mit der gleichen AIBN-Konzentration polymerisiert wurden, zeigt, dass diese sich sowohl im Umsatz, als auch in der mittleren Molmasse und der Dispersität nur unwesentlich unterscheiden. Es werden weder Dispersitäten deutlich unter 1,50 gemessen, noch ein Anstieg der mittleren Molmasse mit dem Umsatz oder eine Verkleinerung der mittleren Molmasse. Auch eine erhöhte Initiatormenge, welche die Bildung eines BHT-Radikals beschleunigen würde, verkleinert die mittlere Molmasse nur minimal, während die Dispersitäten weitestgehend unverändert bleiben. Lediglich der Umsatz erhöht sich, wie es für eine erhöhte Radikalkonzentration zu erwarten ist. Dieses Ergebnis zeigt deutlich, dass kleine Mengen des Inhibitors BHT, wie sie in den verwendeten ViBET-Chargen vorkamen, keinen Einfluss auf die Polymere, die in einer freien radikalischen Polymerisation gebildet werden, haben. Selbst der Umsatz wird nicht beeinträchtigt, obwohl zu erwarten wäre, dass der Inhibitor als Radikalfänger die Radikale, die durch den AIBN-Zerfall gebildet werden, abfängt und dadurch die Propagationsgeschwindigkeit senkt. Somit ist eine BHT-freie Arbeitsweise zwar zu bevorzugen, aber nicht zwingend erforderlich.

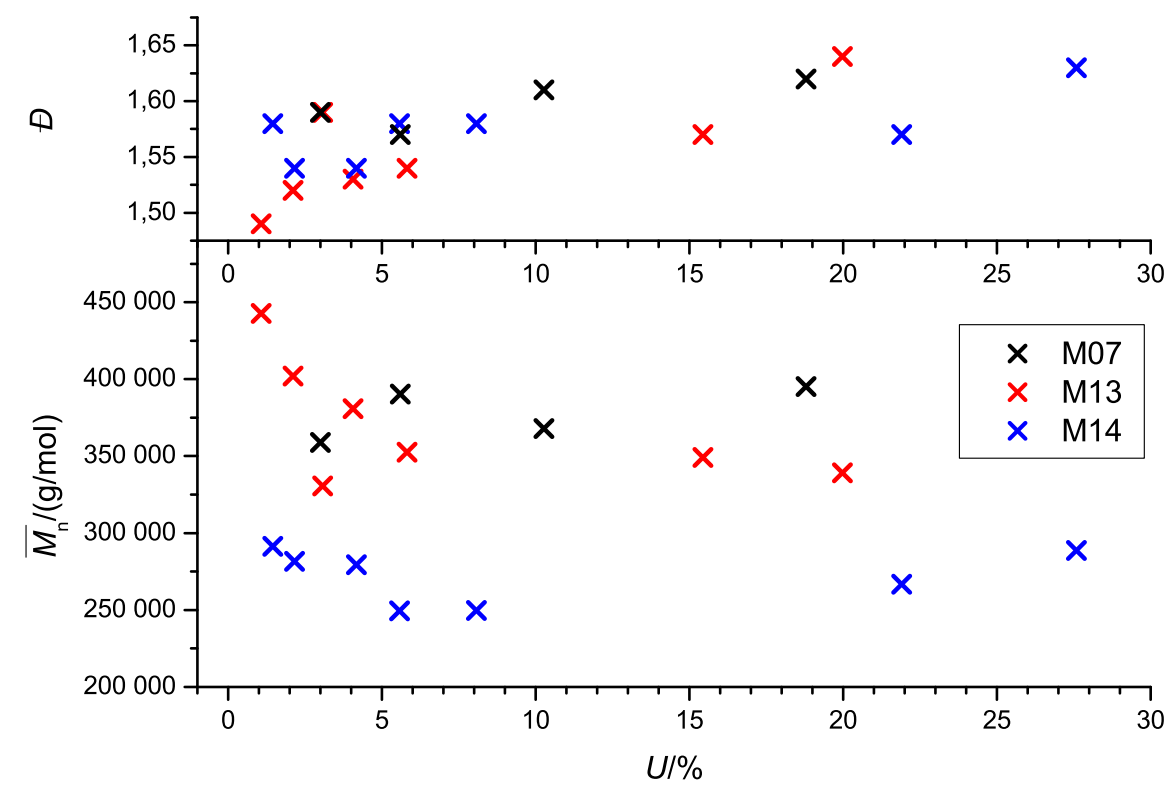

Abbildung 7.7 Dispersitäten und mittlere Molmassen einer Styrol-Polymerisation mit (M13 und M14) und ohne (M07) BHT-Zusatz (siehe Tabelle 7.7 und Tabelle 7.8). 
Tabelle 7.8 Ergebnisse der Styrol-Polymerisationen bei $60^{\circ} \mathrm{C}$. Die Umsätze beziehen sich auf die Comonomermischung.

\begin{tabular}{lcrcrr}
\hline$\#$ & Additiv & $t / \mathrm{h}$ & $\bar{M}_{\mathrm{n}} /(\mathrm{g} / \mathrm{mol})$ & $\oplus$ & $U / \%$ \\
\hline M07A & & 3 & $3,588 \cdot 10^{5}$ & 1,59 & 3,0 \\
M07B & & 6 & $3,903 \cdot 10^{5}$ & 1,57 & 5,6 \\
M07C & & 12 & $3,679 \cdot 10^{5}$ & 1,61 & 10,3 \\
M07D & & 24 & $3,951 \cdot 10^{5}$ & 1,62 & 18,8 \\
\hline M13A & BHT & 1 & $4,427 \cdot 10^{5}$ & 1,49 & 1,1 \\
M13B & BHT & 2 & $4,020 \cdot 10^{5}$ & 1,52 & 2,1 \\
M13C & BHT & 3 & $3,307 \cdot 10^{5}$ & 1,59 & 3,1 \\
M13D & BHT & 4 & $3,809 \cdot 10^{5}$ & 1,53 & 4,1 \\
M13E & BHT & 6 & $3,527 \cdot 10^{5}$ & 1,54 & 5,8 \\
M13F & BHT & 18 & $3,492 \cdot 10^{5}$ & 1,57 & 15,4 \\
M13G & BHT & 24 & $3,392 \cdot 10^{5}$ & 1,64 & 20,0 \\
\hline M14A & BHT & 1 & $2,914 \cdot 10^{5}$ & 1,58 & 1,5 \\
M14B & BHT & 2 & $2,818 \cdot 10^{5}$ & 1,54 & 2,2 \\
M14C & BHT & 3 & $2,794 \cdot 10^{5}$ & 1,54 & 4,2 \\
M14D & BHT & 4 & $2,494 \cdot 10^{5}$ & 1,58 & 5,6 \\
M14E & BHT & 6 & $2,498 \cdot 10^{5}$ & 1,58 & 8,1 \\
M14F & BHT & 18 & $2,667 \cdot 10^{5}$ & 1,57 & 21,9 \\
M14G & BHT & 24 & $2,887 \cdot 10^{5}$ & 1,63 & 27,6 \\
\hline
\end{tabular}




\subsection{Untersuchung der 4-Vinylbenzyl-Abgangsgruppe}

Eine weitere Erklärung für das Verhalten von ViBET in einer radikalischen Polymerisation kann eine Verunreinigung mit dem Dithioester ViBEDiT sein. Dieser Dithioester hat die Struktur eines RAFT-Agens mit einer Methylgruppe als Z-Gruppe und einer 4-Vinylbenzyl-Abgangsgruppe (R-Gruppe). Bedingt durch die Z-Gruppe ist das Intermediat im RAFT-Gleichgewicht vergleichsweise schlecht stabilisiert, wohingegen das Radikal der primären Abgangsgruppe aufgrund des aromatischen Rings relativ gut stabilisiert wird und daher für eine Styrol-Polymerisation geeignet ist (siehe Schema 2.11). Im Vergleich dazu wurde eine Polymerisation mit einem symmetrischen Trithiocarbonat durchgeführt, bei dem die R- und die Z-Gruppe ein 4-Vinylbenzyl-Rest sind. Der 4-Vinylbenzyl-Rest trägt eine terminale Doppelbindung und weist Ähnlichkeit mit substituierten Styrol-Derivaten auf. In der Literatur wurde für solche Substanzen der Begriff Inimer (Initiator-Monomer ${ }^{[121]}$ oder Kettentransfermonomer ${ }^{[122]}$ geprägt. Die Polymerisation wird i.d. R. thermisch selbst-initiiert, wie es bei Styrolderivaten möglich ist $^{[123,124]}$ (daher der Name Inimer) und führt zur Bildung von verzweigten Polymeren, ${ }^{[125]}$ da Radikale das Molekül an zwei Stellen angreifen und in die Polymerkette einbauen können. Kettentransfermonomer bezieht sich auf die gleichzeitige Funktion als Kettentransferagens (vgl. RAFT) und Monomer.

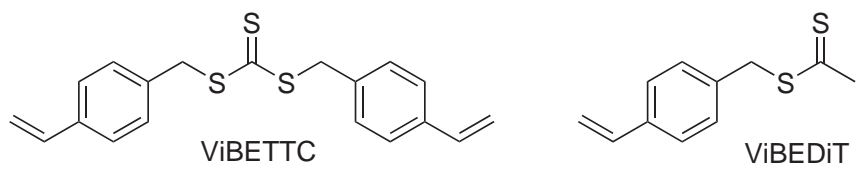

Schema 7.2 Die beiden verwendeten RAFT-Agenzien Bis(4-vinylbenzyl)trithiocarbonat (ViBETTC) und 4-Vinylbenzyldithioacetat (ViBEDiT).

Um die Wirkung der beiden Agenzien (siehe Schema 7.2) optimal vergleichen zu können und Rückschlüsse auf die Qualität der Abgangsgruppe in einer Styrolpolymerisation, den Einfluss der stabilisierenden Gruppe sowie mögliche Vernetzungen ziehen zu können, wurden zwei Polymerisationen mit vergleichbarer Zusammensetzung durchgeführt (siehe Tabelle 7.9).

Die Ergebnisse sind in Tabelle 7.10 aufgeführt und in Abbildung 7.8 grafisch dargestellt. Es fällt auf, dass weder die Umsätze noch die mittleren Molmassen stark voneinander abweichen. Innerhalb der ersten vier Stunden stiegen die Umsätze auf rund $3 \%$ und nach $24 \mathrm{~h}$ betrug der Umsatz bei beiden Polymerisationen ca. 17\%.

Auffällig sind die Dispersitäten, die mit Werten über 3 deutlich größer sind als selbst für 
Styrol-Homopolymerisationen üblich ist. Der Grund dafür ist in Abbildung 7.9 ersichtlich. Dargestellt ist neben dem Polymer auch der niedermolekulare Bereich, in dem verbliebenes RAFT-Agens vorhanden ist. Die Intensität der Polymerpeaks wurde jeweils auf 1 normiert, um einen besseren Vergleich der Intensität des RAFT-Agens-Peaks im niedermolekularen Bereich machen zu können und die zeitliche Veränderung der mittleren Molmasse des Polymers mit der Zeit leichter erkennen zu können.

Bei den ersten drei Proben der Polymerisation mit dem Trithiocarbonat überwiegt der Peak im niedermolekularen Bereich, der dem RAFT-Agens zuzuschreiben ist. Nach $3 \mathrm{~h}$ hat das Polymer deutlich zugenommen und hat nach spätestens $24 \mathrm{~h}$ komplett das Vorgleichgewicht durchlaufen. Die Probe nach $24 \mathrm{~h}$ zeigt außerdem eine Schulter im höhermolekularen Bereich, die auf eine Kopplung der Polymerketten untereinander zurückzuführen ist. Da das RAFT-Agens verhältnismäßig niedrig dosiert worden ist, tritt diese Kopplung erst bei hohen Umsätzen auf.

Die mittleren Molmassen und Dispersitäten, die in Tabelle 7.10 aufgeführt sind, wurden aus dem höhermolekularen Peak, der dem Polymer zuzuschreiben ist, bestimmt. Das unverbrauchte RAFT-Agens, das diese Werte verfälscht hätte, wurde dafür vernachlässigt. Obwohl die mittlere Molmasse zunimmt und diese Tendenz auch in den GPC-Spektren (siehe Abbildung 7.9) ersichtlich ist, handelt es sich bei beiden Substanzen um keine guten RAFT-Agenzien. Während die GPC-Spektren der TrithiocarbonatPolymerisation einen Peak im niedermolekularen Bereich mit unverbrauchtem RAFTAgens zeigt, werden in der Dithioacetat-Polymerisation sogar zwei Peaks im niedermolekularen Bereich detektiert. Da diese Substanz im Gegensatz zu Dithioestern kein UV-Licht der Wellenlänge $310 \mathrm{~nm}$ absorbiert und die Intensität im Laufe der Zeit abnimmt und nach $24 \mathrm{~h}$ nicht mehr sichtbar ist, gehört dieser Peak wahrscheinlich zu Polystyrol-Oligomer (siehe Abbildung 7.10).

Dennoch stimmen die erreichten mittleren Molmassen sehr gut mit den theoretisch berechneten Werten (Formel 2.40) überein (siehe Abbildung 7.8).

Tabelle 7.9 Konzentrationen der Styrol-Polymerisation M15 und M16.

\begin{tabular}{lcccc}
\hline$\#$ & $\begin{array}{c}{[\text { Styrol]/ }} \\
\text { mol-\% }\end{array}$ & Additiv & $\begin{array}{c}\text { [Additiv]/ } \\
\text { mol-\% }\end{array}$ & $\begin{array}{c}{[\text { AIBN]/ }} \\
\text { mol-\% }\end{array}$ \\
\hline M15 & 99,87 & ViBETTC & 0,12 & 0,01 \\
M16 & 99,87 & ViBEDiT & 0,12 & 0,01 \\
\hline
\end{tabular}




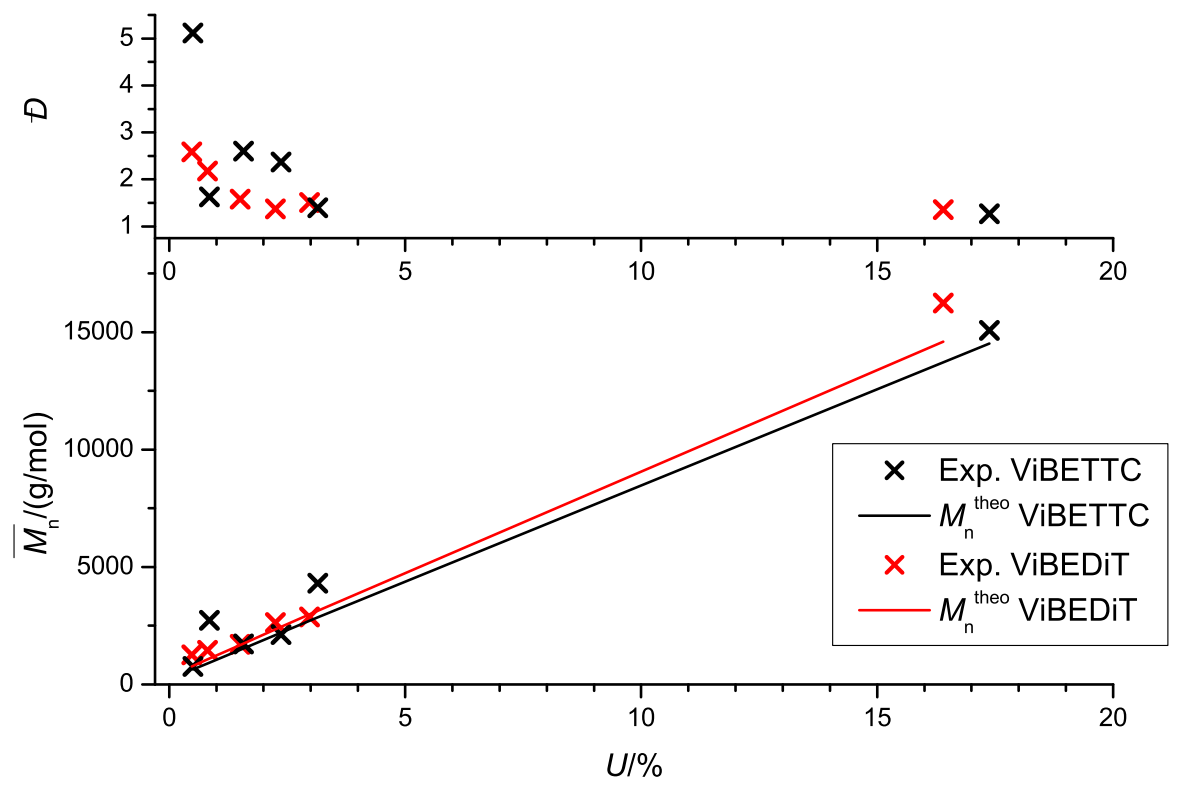

Abbildung 7.8 Dispersitäten und mittlere Molmassen einer Styrol-Polymerisation mit ViBETTC und ViBEDiT (siehe Tabelle 7.9 und Tabelle 7.10).

Tabelle 7.10 Ergebnisse der Styrol-Polymerisationen bei $60^{\circ} \mathrm{C}$. Die Umsätze beziehen sich auf Styrol.

\begin{tabular}{lcccccc}
\hline$\#$ & Additiv & $t / \mathrm{h}$ & $\bar{M}_{\mathrm{n}} /(\mathrm{g} / \mathrm{mol})$ & $\bar{M}_{\mathrm{n}}^{\text {theo }} /(\mathrm{g} / \mathrm{mol})$ & $\oplus$ & $U / \%$ \\
\hline M15A & ViBETTC & 0,5 & $1,26 \cdot 10^{3}$ & $7,71 \cdot 10^{2}$ & 2,59 & 0,5 \\
M15B & ViBETTC & 1 & $1,45 \cdot 10^{3}$ & $1,070 \cdot 10^{3}$ & 2,18 & 0,8 \\
M15C & ViBETTC & 2 & $1,71 \cdot 10^{3}$ & $1,684 \cdot 10^{3}$ & 1,58 & 1,5 \\
M15D & ViBETTC & 3 & $2,64 \cdot 10^{3}$ & $2,342 \cdot 10^{3}$ & 1,37 & 2,2 \\
M15E & ViBETTC & 4 & $2,87 \cdot 10^{3}$ & $2,987 \cdot 10^{3}$ & 1,51 & 3,0 \\
M15F & ViBETTC & 24 & $1,62 \cdot 10^{4}$ & $1,460 \cdot 10^{4}$ & 1,36 & 16,4 \\
\hline \multirow{2}{*}{ M16A } & ViBEDiT & 0,5 & $7,66 \cdot 10^{2}$ & $6,36 \cdot 10^{2}$ & 5,12 & 0,5 \\
M16B & ViBEDiT & 1 & $2,73 \cdot 10^{3}$ & $9,25 \cdot 10^{2}$ & 1,63 & 0,8 \\
M16C & ViBEDiT & 2 & $1,73 \cdot 10^{3}$ & $1,538 \cdot 10^{3}$ & 2,61 & 1,6 \\
M16D & ViBEDiT & 3 & $2,12 \cdot 10^{3}$ & $2,207 \cdot 10^{3}$ & 2,37 & 2,4 \\
M16E & ViBEDiT & 4 & $4,31 \cdot 10^{3}$ & $2,858 \cdot 10^{3}$ & 1,40 & 3,2 \\
M16F & ViBEDiT & 24 & $1,51 \cdot 10^{4}$ & $1,452 \cdot 10^{4}$ & 1,27 & 17,4 \\
\hline
\end{tabular}



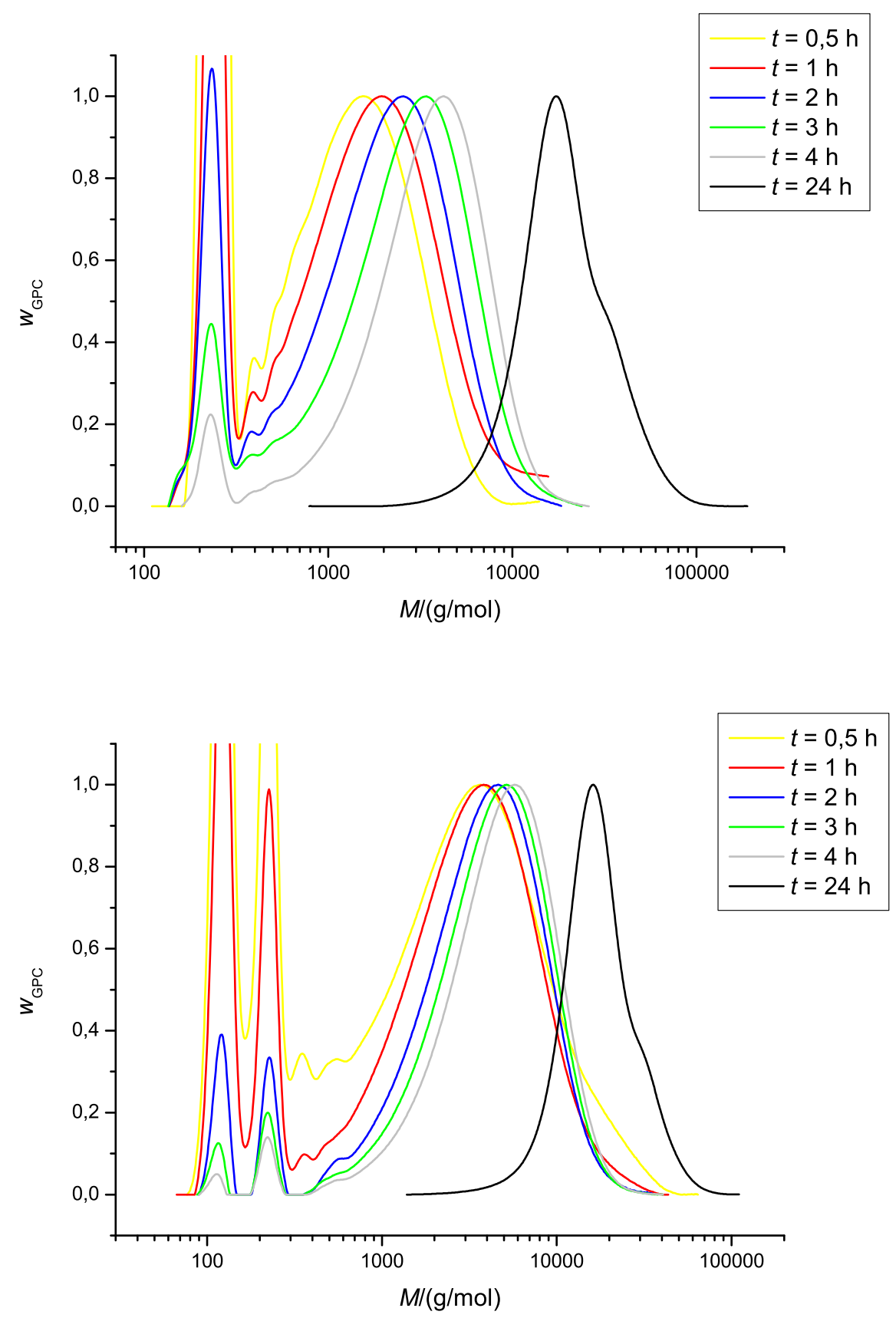

Abbildung 7.9 Zeitlicher Verlauf der Molmassenverteilungen der StyrolPolymerisation mit ViBETTC (oben) bzw. ViBEDiT (unten; siehe Tabelle 7.9 und Tabelle 7.10). 


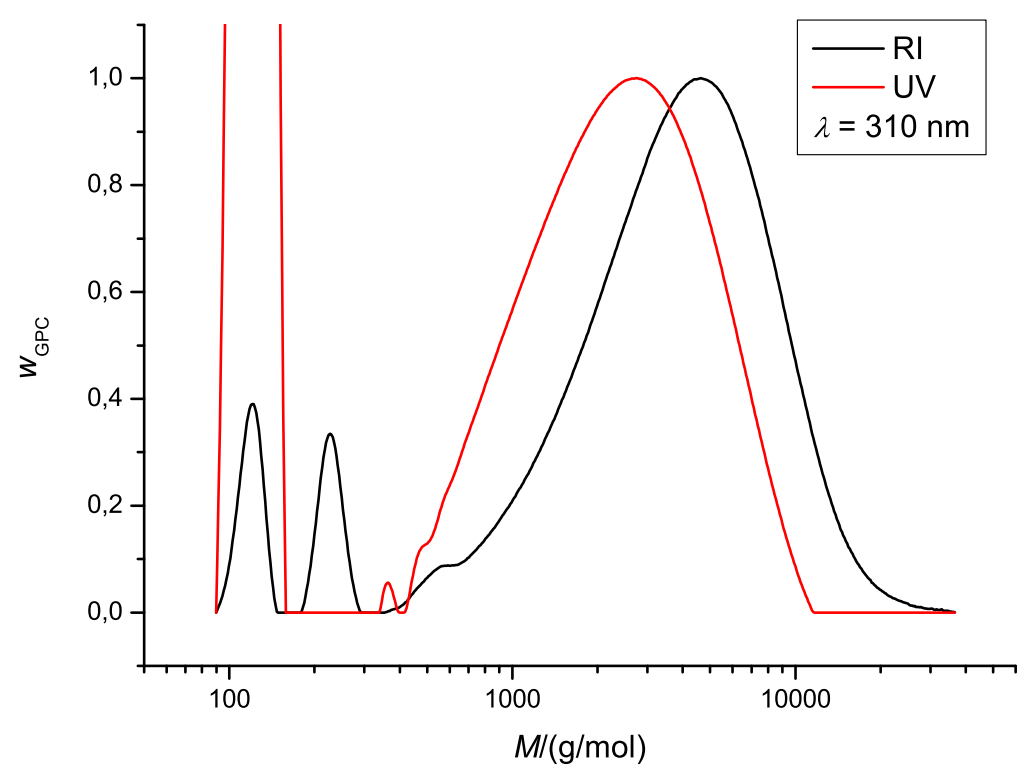

Abbildung 7.10 Molmassenverteilungen der Styrol-Polymerisation mit ViBEDiT M16C nach $2 \mathrm{~h}$ bei $60^{\circ} \mathrm{C}$ (siehe Tabelle 7.9 und Tabelle 7.10).

\subsection{Styrol-ViBET-Copolymerisationen}

Die Zusammensetzung eines ViBET-Styrol-Copolymers lässt sich auf verschiedene Arten bestimmen, die verschiedene Vor- und Nachteile haben.

Eine Möglichkeit ist die NMR-Spektroskopie. Dafür muss sich das Copolymer in einem Lösungsmittel lösen, das ein Restprotonensignal liefert, welches sich mit keinem der Polymerprotonensignale überlagert. Durch Integration der Methylenprotonen oder der Methylprotonen des ViBET-Moleküls und einen Vergleich mit den aromatischen Protonen, die sich aus ViBET und Styrol addieren, lassen sich Rückschlüsse auf die Polymerzusammensetzung ziehen. Da die Protonen der Methylgruppe eine ähnliche Verschiebung wie die Protonen der Polymerkette haben und somit von diesen überlagert werden, wurden die Protonen der Methylengruppe verwendet.

Eine weitere Möglichkeit stellt die Elementaranalyse dar. Der Schwefelgehalt erlaubt direkt Rückschlüsse auf den ViBET-Gehalt, da Styrol schwefelfrei ist. Wenn die Messwerte stark streuen, kann darüber hinaus aus dem Kohlenstoffgehalt, der sich bei den beiden Monomeren stark unterscheidet, die Polymerzusammensetzung berechnet werden. 
Um das Copolymerisationsverhalten beurteilen zu können, wurden zwei Polymerisationen mit relativ hohem ViBET-Gehalt durchgeführt, damit der Schwefelgehalt für die Elementaranalyse ausreichend hoch ist. Das Polymer wurde in einem geeigneten Lösungsmittel (hier: Methanol) ausgefällt, abfiltriert und getrocknet, um Monomerreste zu entfernen. Dieses Vorgehen ist nötig, da ViBET im Gegensatz zu Styrol selbst unter vermindertem Druck nur unzureichend abdampft.

Aufgrund der bekannten Problematik, dass in einer Styrol-ViBET-Copolymerisation tendenziell kurze Ketten gebildet werden, wurden Polymerproben nach 24, 48 und $72 \mathrm{~h}$ entnommen. Die Polymere, die einen Anteil von 2 mol-\% ViBET in der Polymermischung enthalten, wurden hinsichtlich ihrer Zusammensetzung untersucht.

Tabelle 7.11 Konzentrationen der Styrol-Polymerisation M17 und M18.

\begin{tabular}{lcccc}
\hline$\#$ & $\begin{array}{c}\text { [Styrol]/ } \\
\text { mol- } \%\end{array}$ & Additiv & $\begin{array}{r}\text { [Additiv]/ } \\
\text { mol-\% }\end{array}$ & $\begin{array}{c}\text { [AIBN]/ } \\
\text { mol-\% }\end{array}$ \\
\hline M17 & 89,97 & ViBET & 10,00 & 0,03 \\
M18 & 97,99 & ViBET & 1,98 & 0,03 \\
\hline
\end{tabular}

Die mittleren Molmassen und Dispersitäten, die in den beiden Polymerisationen erreicht wurden, wurden gegen die Polymerisationszeit aufgetragen (siehe Abbildung 7.11), da die Umsätze nicht bestimmt werden konnten.

Der Trend, dass eine hohe ViBET-Konzentration mit einer kleineren mittleren Molmasse einhergeht, ist wieder deutlich zu beobachten. Die Polymere, die identische Polymerisationszeiten besitzen, unterscheiden sich in ihrer mittleren Molmasse nahezu um eine Potenz. Neu und auf den ersten Blick ungewöhnlich sind die Dispersitäten. Während in ersten Experimenten mit ViBET Polymere mit Dispersitäten deutlich kleiner als 1,50 synthetisiert werden konnten, haben die Copolymere der Polymerisationen M18A - C ungewöhnlich hohe Dispersitäten. Die Molmassenverteilungen zeigen den Grund dafür (siehe Abbildung 7.12). Abgesehen von dem Polymer, das in der Polymerisation M17A hergestellt wurde, zeigen alle Polymere eine Schulter im höhermolekularen Bereich. Das Polymer, das aus der Polymerisation M18C stammt, zeigt sogar eine zweite Schulter, die sich bei dem Polymer aus der Polymerisation M18B bereits erahnen lässt. Es gibt unterschiedliche Möglichkeiten, wodurch diese Schultern, die aus Polymer mit einer höheren mittleren Molmasse bestehen, zustande kommen. Auf den ersten Blick unerwartet scheint die Tatsache, dass ausschließlich in der Polymerisation M17A kein Polymer mit einem höhermolekularen Anteil gebildet wurde. Die Monomermischung 
Tabelle 7.12 Ergebnisse der Styrol-Polymerisationen bei $60^{\circ} \mathrm{C}$. ${ }^{a}$ Umsatz konnte nicht bestimmt werden.

\begin{tabular}{lccccc}
\hline$\#$ & {$[$ ViBET] } & $t / \mathrm{h}$ & $\bar{M}_{\mathrm{n}} /(\mathrm{g} / \mathrm{mol})$ & $Đ$ & $U / \%$ \\
\hline M17A & 10,00 mol-\% & 24 & $1,110 \cdot 10^{4}$ & 1,29 & 16,2 \\
M17B & 10,00 mol-\% & 48 & $2,009 \cdot 10^{4}$ & 1,32 & 26,5 \\
M17C & 10,00 mol-\% & 72 & $2,258 \cdot 10^{4}$ & 1,37 & 30,2 \\
\hline M18A $^{2}$ & 1,98 mol-\% & 24 & $8,316 \cdot 10^{4}$ & 1,49 & 33,0 \\
M18B $^{a}$ & 1,98 mol-\% & 48 & $1,266 \cdot 10^{5}$ & 1,80 & \\
M18C $^{a}$ & 1,98 mol-\% & 72 & $1,837 \cdot 10^{5}$ & 2,11 & \\
\hline
\end{tabular}

der Polymerisationen M17A - C enthielt 10 mol-\% ViBET und es liegt nahe, dass das höhermolekulare Polymer durch das verwendete Comonomer ViBET gebildet wird, da reine Polystyrole keine derartige Molmassenverteilung aufweisen.

Die geringen Mengen höhermolekulares Polymer, die in Form dieser Schultern auftreten, werden durch vereinzelte, langkettige Polymere hervorgerufen, die durch die Reaktion zweier Polymere miteinander entstehen. Dabei ist es möglich, dass zwei MakroRadikale in einem Kombinationsmechanismus terminieren oder dass bereits unter Polymerisationsbedingungen (erhöhte Temperatur und Bildung von Radikalen) der Thioester des ViBET-Moleküls angegriffen und gespalten wird. Das daraus resultierende schwefelzentrierte Radikal kann mit einer anderen Polymerkette unter Ausbildung eines Netzknotenpunktes reagieren. Da eine Reduktion mit Zink ${ }^{[126]}$ keine Änderung der Molmassenverteilung bewirkte, kann das Vorhandensein von Disulfidbrücken ausgeschlossen werden. Dennoch ist eine Verknüpfung zweier Polymerketten über ein verbrückendes Schwefelatom möglich.

Theoretisch wird dieses Verhalten bei höherer ViBET-Konzentration verstärkt erwartet. Dass trotzdem die Polymere aus der Polymerisation M18, die eine vergleichsweise niedrigere ViBET-Konzentration besitzen, stärker zu Nebenreaktionen tendieren, liegt an dem Monomerumsatz. Der Styrol-Umsatz der Polymerisation M18A ist bereits gröBer als der Styrol-Umsatz der Polymerisation M17C, obwohl die Polymerisationszeit nur $24 \mathrm{~h}$ statt $72 \mathrm{~h}$ betrug. Für den Fall, dass ein Monomer bevorzugt eingebaut wird, verschiebt sich das Verhältnis von Styrol zu ViBET im Polymer im Vergleich zur Monomermischung und es kommt zu einem sogenannten "composition drift". Die Wahrscheinlichkeit, dass ein ViBET-Molekül attackiert wird, ändert sich und somit auch die Wahrscheinlichkeit für Nebenreaktionen. 


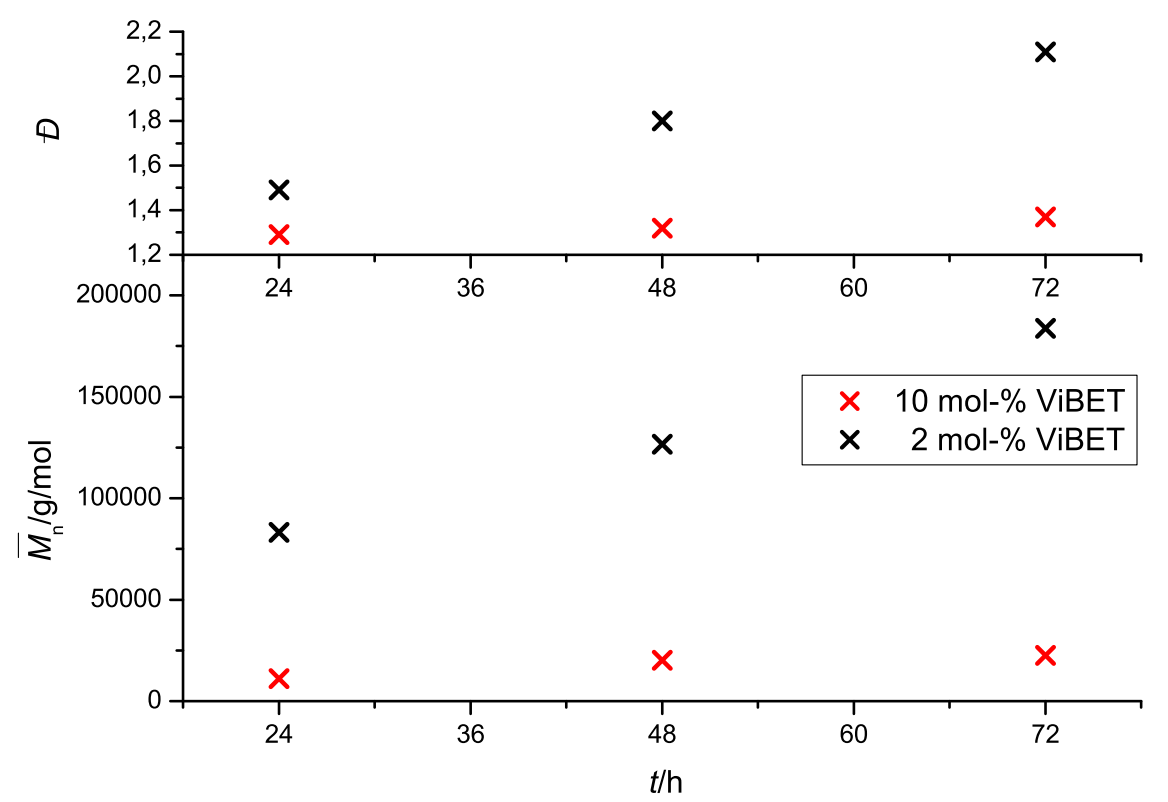

Abbildung 7.11 Mittlere Molmasse und Dispersität der Styrol-ViBETCopolymerisationen bei $60^{\circ} \mathrm{C}$ in Abhängigkeit von der Polymerisationszeit (siehe Tabelle 7.11 und Tabelle 7.12).

In Tabelle 7.12 sind neben den zahlengemittelten Molmassen noch die Peakmaxima $M_{\mathrm{p} 1}$ und $M_{\mathrm{p} 2}$ dargestellt, sofern sie bestimmt werden konnten. Bei den Polymeren der Polymerisationen M17B bzw. M18C ließ sich die Position des zweiten bzw. dritten Maximums nicht eindeutig bestimmen, bei M18A konnte die Position des zweiten Maximums lediglich grob abgeschätzt werden. Die Ergebnisse in Tabelle 7.13 bestätigen die Vermutung, dass die Schulter bzw. der Peak im höhermolekularen Bereich durch die Kopplung zweier Polymerketten zustande kommt, da die Molmasse $M_{\mathrm{p} 2}$ circa doppelt so groß ist wie die Molmasse $M_{\mathrm{p} 1}$. 

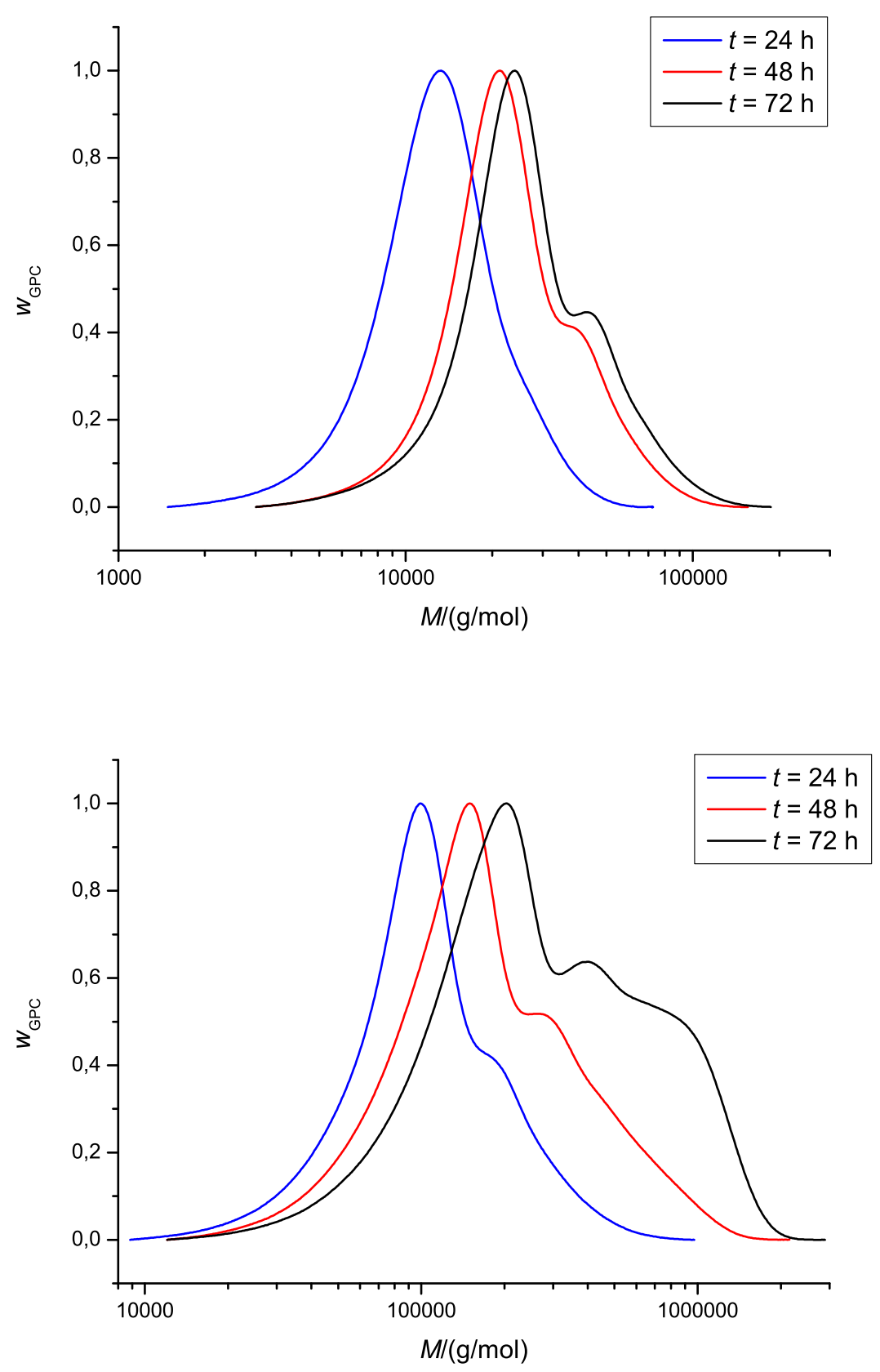

Abbildung 7.12 Zeitlicher Verlauf der Molmassenverteilungen der Styrol-ViBETCopolymerisationen mit 10 mol-\% ViBET im Monomer (oben) bzw. 2 mol-\% ViBET im Monomer (unten; siehe Tabelle 7.11 und Tabelle 7.12). 
Tabelle 7.13 Ergebnisse der Styrol-Polymerisationen bei $60{ }^{\circ} \mathrm{C} .{ }^{a} M_{\mathrm{p} 2}$ Circa-Angabe.

\begin{tabular}{lccc}
\hline$\#$ & $\bar{M}_{\mathrm{n}} /(\mathrm{g} / \mathrm{mol})$ & $M_{\mathrm{p} 1} /(\mathrm{g} / \mathrm{mol})$ & $M_{\mathrm{p} 2} /(\mathrm{g} / \mathrm{mol})$ \\
\hline M17A & $1,110 \cdot 10^{4}$ & $1,322 \cdot 10^{4}$ & \\
M17B & $2,009 \cdot 10^{4}$ & $2,117 \cdot 10^{4}$ & \\
M17C & $2,258 \cdot 10^{4}$ & $2,393 \cdot 10^{4}$ & $4,262 \cdot 10^{4}$ \\
\hline M18A $^{a}$ & $8,316 \cdot 10^{4}$ & $9,705 \cdot 10^{4}$ & $1,9 \cdot 10^{4}$ \\
M18B $^{4}$ & $1,266 \cdot 10^{5}$ & $1,502 \cdot 10^{5}$ & $2,622 \cdot 10^{5}$ \\
M18C & $1,837 \cdot 10^{5}$ & $2,034 \cdot 10^{5}$ & $3,988 \cdot 10^{5}$ \\
\hline
\end{tabular}

\subsubsection{Polymerzusammensetzung}

Die Polymere, die aus den Polymerisationen M18B und M18C stammen, wurden gefällt und anschließend mittels NMR-Spektroskopie und Elementaranalyse auf ihre Zusammensetzung untersucht. Hierbei wurden bewusst langkettige Polymere ausgewählt, da diese für eine Weiterverarbeitung im Rahmen der Kooperation besonders interessant sind und sich gut ausfällen lassen. Die ViBET-Konzentration von 2 mol-\% in der Monomermischung lässt ein Polymer mit einer ViBET-Konzentration bis zu 5 mol-\% erwarten, wie es angestrebt wurde.

Es wurden zwei bzw. drei Proben der Polymere hinsichtlich ihres Kohlenstoff-, Wasserstoff- und Schwefelgehaltes analysiert. Während der Kohlenstoff- und Wasserstoffgehalt konsistente Messergebnisse lieferten, schwankte der Schwefelgehalt stark. Da Styrol und ViBET sich nicht nur im Schwefel-, sondern auch im Kohlenstoffgehalt deutlich unterscheiden, wurde die Konzentration des Comonomers ViBET aus beiden Messgrößen berechnet, wobei die Ergebnisse sich deutlich unterscheiden. Während die Berechnung über den Kohlenstoffgehalt ViBET-Konzentrationen im Bereich 2-3 mol-\% ergibt, resultieren aus dem Schwefelgehalt Konzentrationen im Bereich von $4-11 \mathrm{~mol}-\%$.

Darüber hinaus wurden die Polymere in THF- $\mathrm{d}_{8}$ gelöst und mittels NMRSpektroskopie untersucht. Der ViBET-Gehalt beträgt laut NMR-Spektroskopie 6,3\% (siehe Tabelle 7.14) und liegt damit über dem Ergebnis für die Elementaranalyse.

Der exakte ViBET-Gehalt kann nicht bestimmt werden, da die Messwerte stark streuen und die unterschiedlichen Methoden unterschiedliche Ergebnisse liefern. Dabei ist wahrscheinlich die Analyse des Kohlenstoffgehaltes und die daraus berechnete ViBETKonzentration am genauesten, da sie den geringsten Schwankungen unterworfen ist. 
Die Berechnung aus den Integralen der NMR-Spektren kann aufgrund einer verlängerten Relaxationszeit von Polymerprotonen trotz angepasster Messparameter ungenau sein. Die Polymere enthalten wahrscheinlich einen Schwefelgehalt, der im Rahmen des angestrebten Bereichs von 2 - 5 mol-\% liegt.

Tabelle 7.14 Ergebnisse der Elementaranalyse der Styrol-ViBET-Copolymere M18B und M18C. ${ }^{a}$ Bestimmt über den Schwefelgehalt. ${ }^{b}$ Bestimmt über den Kohlenstoffgehalt. ${ }^{c}$ Bestimmt über NMR-Spektroskopie.

\begin{tabular}{lcccccc}
\hline$\#$ & $\begin{array}{r}\mathrm{C} / \\
\%\end{array}$ & $\begin{array}{r}\mathrm{H} / \\
\%\end{array}$ & $\begin{array}{c}\mathrm{S} / \\
\%\end{array}$ & $\begin{array}{c}\text { [ViBET }^{a} / \\
\text { mol-\% }\end{array}$ & $\begin{array}{c}\text { [ViBET] }^{b} / \\
\text { mol-\% }\end{array}$ & $\begin{array}{c}\text { [ViBET] }^{c} / \\
\text { mol-\% }\end{array}$ \\
\hline Styrol & 92,26 & 7,74 & & & & \\
ViBET & 68,71 & 6,29 & 16,68 & & & \\
\hline M18B-I & 90,98 & 7,73 & 2,41 & 8,4 & 3,0 & 6,3 \\
M18B-II & 91,15 & 7,70 & 1,11 & 3,7 & 2,6 & \\
M18B-III & 90,90 & 7,55 & 1,19 & 4,0 & 3,1 & \\
\hline M18C-I & 91,34 & 7,80 & 3,09 & 11,0 & 2,1 & 6,3 \\
M18C-II & 91,24 & 7,67 & 1,15 & 3,9 & 2,3 & \\
\hline
\end{tabular}

\subsection{Butadien-ViBET-Copolymerisationen}

Ziel dieser Arbeit ist die Integration von Monomeren mit potenziell vulkanisierbaren Gruppen in Polybutadien, z. B. in Form eines Butadien-ViBET-Copolymers. Aufgrund der Erfahrungen aus den Styrol-Polymerisationen wurden verhältnismäßig lange Polymerisationszeiten gewählt. Neben einer Langzeitpolymerisation mit $48 \mathrm{~h}$ Reaktionszeit (M19) wurde eine Polymerisation mit einer regelmäßigen Probenentnahme durchgeführt (M20). Die verwendeten Konzentrationen sind in Tabelle 7.15 aufgeführt. Im Gegensatz zu den Styrol-Polymerisationen kann hierbei aufgrund des gasförmigen Monomers nicht auf das Lösungsmittel verzichtet werden. Die Auswirkungen, die ViBET auf eine Styrol-Polymerisation hat, konnten auch hier beobachtet werden. Die Polymerketten wachsen äußerst langsam, die mittlere Molmasse nimmt im Laufe der Zeit zu und die Dispersitäten sind mit Werten unter 1,50 für Butadien-Polmerisationen relativ niedrig. Nach $48 \mathrm{~h}$ beträgt die mittlere Molmasse ca. $5000 \mathrm{~g} / \mathrm{mol}$, was bei einer ButadienMolmasse von 54,09 g/mol einer Anlagerung von ca. 2 Monomereinheiten pro Stunde 
entspricht. Eine geringere ViBET-Konzentration kann nicht gewählt werden, da dann die ViBET-Konzentration im Polymer zu gering ist. Stattdessen könnte eine höhere Polymerisationstemperatur (z. B. $80^{\circ} \mathrm{C}$ ) verwendet werden, bei der die Propagationsgeschwindigkeit höher ist. In diesem Zusammenhang sollte der Initiator ausgetauscht werden, da die Halbwertszeit von AIBN bei $80^{\circ} \mathrm{C}$ für Polymerisationen über mehrere Tage zu klein ist. Als Alternative bietet sich z. B. ACCN an, dessen Halbwertszeit bei $80^{\circ} \mathrm{C}$ fast exakt der Halbwertszeit von AIBN bei $60^{\circ} \mathrm{C}\left(10,5 \mathrm{~h}^{[54]}\right)$ entspricht.

Tabelle 7.15 Konzentrationen der Butadien-ViBET-Copolymerisationen in Toluol bei $60^{\circ} \mathrm{C}$.

\begin{tabular}{lcccc}
\hline$\#$ & $\begin{array}{c}\text { [Butadien]/ } \\
\text { mol-\% }\end{array}$ & $\begin{array}{r}{[\text { ViBET]/ }} \\
\text { mol-\% }\end{array}$ & $\begin{array}{r}\text { [AIBN]/ } \\
\text { mol-\% }\end{array}$ & $\begin{array}{c}\text { [Toluol]/ } \\
\text { mol-\% }\end{array}$ \\
\hline M19 & 36,792 & 0,639 & 0,010 & 62,559 \\
M20 & 29,137 & 0,318 & 0,003 & 62,559 \\
\hline
\end{tabular}

Um den Einbau von ViBET in das Copolymer verifizieren zu können, wurde die UVAbsorption der Probe bei einer Wellenlänge von $295 \mathrm{~nm}$ mittels GPC bestimmt. Bei dieser Wellenlänge absorbiert reines Polybutadien nicht, aber der Thioester. Da zusätzlich zu dem RI-Detektor auch der UV-Detektor Polymer detektiert, wurde das gewünschte Copolymer erfolgreich synthetisiert. Um Hinweise auf die Zusammensetzung des Copolymers erhalten zu können, werden längerkettige Polymere benötigt, die ausgefällt und mittels Elementaranalyse charakterisiert werden können. Nur so lassen sich ViBET-Reste, die das Ergebnis verfälschen würden, entfernen.

Tabelle 7.16 Ergebnisse der Butadien-ViBET-Copolymerisationen bei $60^{\circ} \mathrm{C}$.

${ }^{a}$ Bezogen auf den Gesamtmonomergehalt.

\begin{tabular}{lccccc}
\hline$\#$ & [ViBET $^{a}$ & $t / \mathrm{h}$ & $\bar{M}_{\mathrm{n}} /(\mathrm{g} / \mathrm{mol})$ & $Ð$ & $U / \%$ \\
\hline M19 & $1,7 \mathrm{~mol}-\%$ & 48 & $5,052 \cdot 10^{3}$ & 1,47 & 9,4 \\
\hline M20A & $1,1 \mathrm{~mol}-\%$ & 24 & $5,323 \cdot 10^{3}$ & 1,26 & 5,5 \\
M20B & $1,1 \mathrm{~mol}-\%$ & 30 & $5,203 \cdot 10^{3}$ & 1,47 & 6,2 \\
M20C & $1,1 \mathrm{~mol}-\%$ & 48 & $6,265 \cdot 10^{3}$ & 1,48 & 6,9 \\
\hline
\end{tabular}




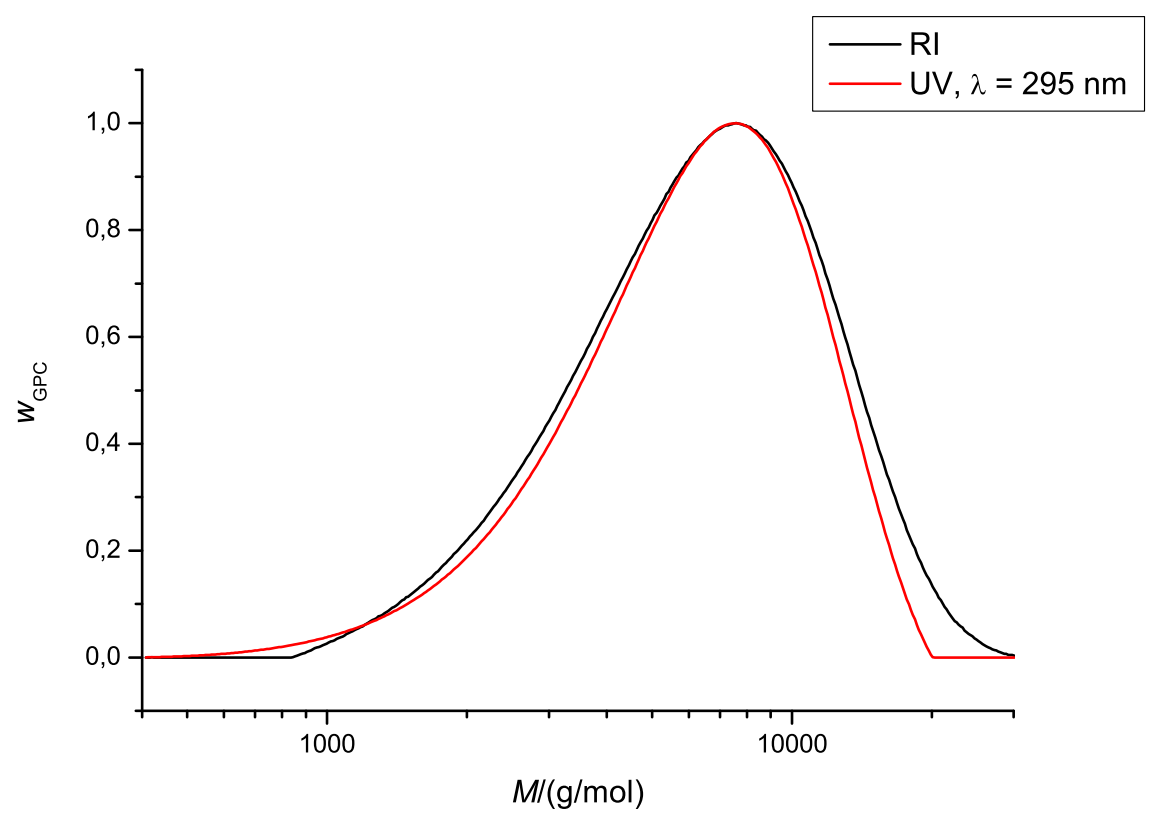

Abbildung 7.13 Molmassenverteilung der Butadien-ViBET-Copolymerisation M19 mit 1,7 mol-\% ViBET im Monomer (siehe Tabelle 7.15 und Tabelle 7.16).

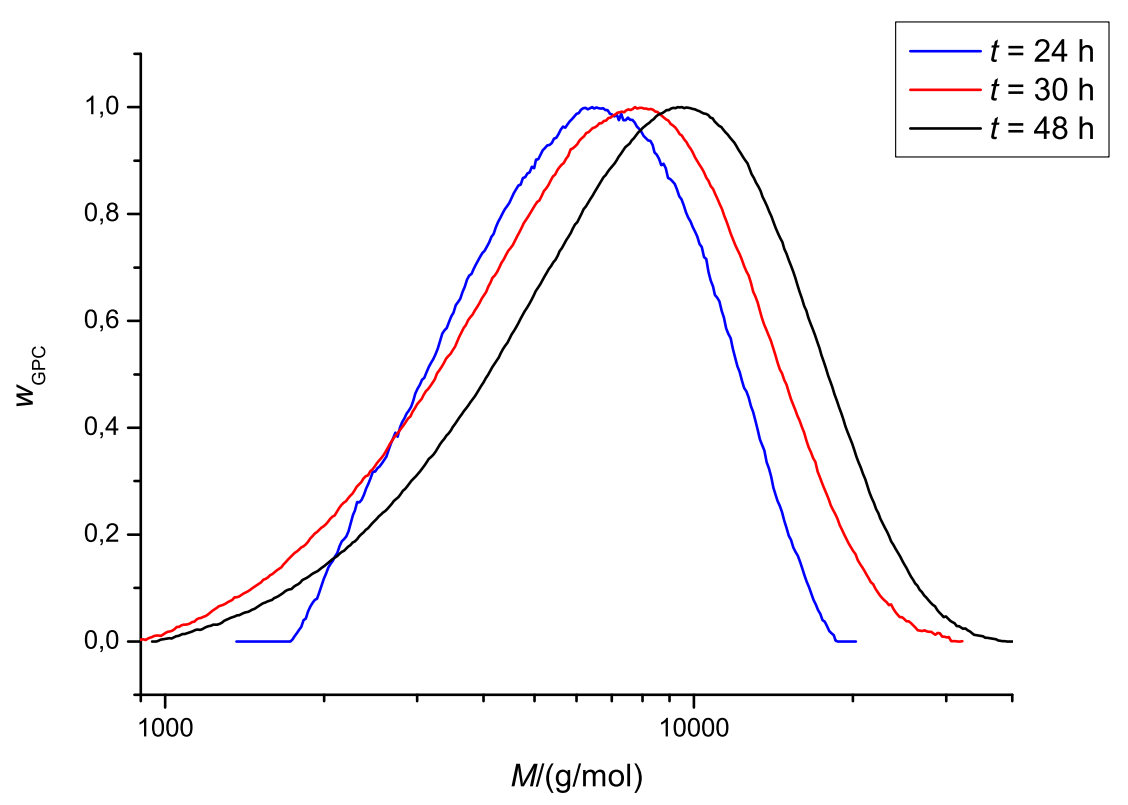

Abbildung 7.14 Molmassenverteilung der Butadien-ViBET-Copolymerisationen M20A-C mit 1 mol-\% ViBET im Monomer (siehe Tabelle 7.15 und Tabelle 7.16). 


\subsection{Fazit}

4-Vinylbenzylthioacetat ist ein Monomer, das sich leicht synthetisieren lässt, aber in der Anwendung Probleme bereitet. Während die Bildung von Polymer mit einer niedrigeren Dispersität, als es für eine Styrol- oder Butadien-Homopolymerisation üblich ist, ein positiver Nebeneffekt ist, erweist sich die Reduzierung der mittleren Molmasse und des Monomerumsatzes, die in einer Polymerisation mit ViBET erreicht werden, als problematisch. Da der Umsatz in einer Styrol-ViBET-Copolymerisation mit 2 mol-\% ViBET nach $24 \mathrm{~h}$ bereits $33 \%$ beträgt, während er in einer Polymerisation mit fünf mal höherer ViBET-Konzentration erst bei 16\% liegt, kann davon ausgegangen werden, dass sich die Propagationsgeschwindigkeit durch die Bildung eines retardierenden Radikals verringert. Es bildet sich ein Intermediat, das für eine gewisse Zeit stabil ist, kein Monomer anlagert, nicht signifikant terminiert wird und somit sowohl zu einem geringeren Umsatz als auch zu kleineren mittleren Molmassen führt.

Der Vergleich mit den ViBET-ähnlichen Substanzen hat gezeigt, dass es sich hierbei um eine Besonderheit des Moleküls handelt. Der reine Sauerstoff-Ester 4-Vinylbenzylacetat (ViBA) beeinflusst die Polymerisation in keinster Weise. Ein Thioacetat ohne aromatischen Rest (Ethylthioacetat, ETA) führt zu einer Verringerung der mittleren Molmassen, beeinflusst aber den Umsatz nur unwesentlich, was für einen Einfluss des Thioacetat-Restes in Form einer Übertragungsreaktion spricht. Der Dithioester 4Vinylbenzyldithioacetat (ViBEDiT) zeigt das zu erwartende Verhalten eines RAFTAgens. Den größten Einfluss hat neben 4-Vinylbenzylthioacetat (ViBET) die gesättigte Variante 4-Ethylbenzylthioacetat (EBET). Während in der Polymerisation mit EBET der Umsatz stark einbricht, aber die mittlere Molmasse verhältnismäßig stark ansteigt, werden in einer ViBET-Polymerisation größere Monomerumsätze und vergleichsweise niedrigere mittlere Molmassen gemessen.

Die entscheidende Rolle spielt somit der Thioester, der an einen Phenylrest gebunden ist. Der Substituent in para-Position hat ebenfalls einen Einfluss auf die Polymerisation, was zweierlei Gründe hat: Bei ViBET handelt es sich um ein Styrol-Derivat, welches somit einpolymerisieren kann (und auch soll). Dies ist bei EBET nicht der Fall. Darüber hinaus besitzt das ViBET-Molekül ein größeres delokalisiertes $\pi$-Elektronensystem, welches ein Radikal sehr gut stabilisieren kann. So kann z. B. ein ungepaartes Elektron eines Makroradikals abstrahiert werden und so dem Propagationsschritt entzogen werden. Nach kurzer Zeit erfolgt die Übertragung auf ein Makroradikal, gefolgt von Kettenwachstumsschritten.

Für die technische Anwendung sind hohe Molmassen, besonders in der Butadien- 
Polymerisation, erstrebenswert, da diese sich leichter zusammen mit dem hochmolekularen Naturkautschuk verarbeiten lassen. Lange Polymerisationszeiten in einer StyrolViBET-Copolymerisation führen zwar zu einer Erhöhung der mittleren Molmassen, aber gleichzeitig auch zu ersten Quervernetzungen der Polymere. Eine Verringerung der ViBET-Konzentration kann keine Abhilfe schaffen, da mit einer geringeren Konzentration der Umsatz ansteigt und vermutlich aufgrund der erhöhten Viskosität die Quervernetzungen noch früher eintreten. Die Molmassenverteilungen zeigen, dass es in dem gewählten System zur Bildung von 1 oder 2 Netzknotenpunkten kommt, da sich Polymerpeaks mit doppelter bis dreifacher Molmasse des Hauptpeaks bilden. Da die Konzentratrion des Comonomers ViBET im Polymer bei mindestens $2 \%$ liegt und somit jedes 50. Monomermolekül eine funktionelle Gruppe trägt, stehen bei einer mittleren Molmasse von $2 \cdot 10^{5} \mathrm{~g} / \mathrm{mol}$ immer noch genügend vulkanisierbare Gruppe zur Verknüpfung während der Vulkanisation zur Verfügung. Darüber hinaus führt eine anfängliche Vernetzung während der Polymerisation zu einer Steigerung der mittleren Molmasse und behebt dadurch o. g. Problem.

In der Literatur ist eine Styrol-ViBET-Copolymerisation bekannt, bei der aus 10\% ViBET und $90 \%$ Styrol in der Monomermischung $80 \%$ Umsatz ein Polymer entsteht, dessen ViBET-Anteil bei 12,6\% liegt (Elementaranalyse). ${ }^{[104]}$ Diese Anreicherung von ViBET im Polymer konnte hier ebenfalls beobachtet werden, die die Elementaranalysen der Polymere mit $2 \%$ ViBET in der Monomermischung zeigen.

Da die Butadien-Polymerisationen in Lösung durchgeführt werden, ist die Wahrscheinlichkeit für Quervernetzungen dort geringer. Die sehr kleinen Molmassen, die in den Butadienpolymerisationen erhalten werden, können durch den Zusatz eines weiteren Comonomers mit einer größeren Propagationsgeschwindigkeit (z. B. Styrol) sowie eine höhere Polymerisationstemperatur in Kombination mit einem anderen Initiator, der für die erhöhte Temperatur geeignet ist, umgangen werden. 


\section{Copolymere mit 4-Vinylbenzylbenzolthiosulfonat}

Neben ViBET wurde ein zweites Monomer, 4-Vinylbenzylbenzolthiosulfonat (ViBSuT), synthetisiert. Obwohl es nicht möglich war, dieses Monomer rein zu isolieren, wurde eine Copolymerisation mit dem Rohproduktgemisch A03 durchgeführt, um den eventuellen Einfluss der Verunreinigungen auf eine radikalische Polymerisation zu untersuchen. Die Zusammensetzung des Comonomers betrug 85,7 mol-\% ViBSuT 26, 4,7 mol-\% des Hydrolyseprodukts 27 und 9,6 mol-\% des Dimers 28. Die erfolgreiche Verwendung des Monomers ohne weitere Aufreinigung würde eine erhebliche Kosten- und Zeitersparnis darstellen.<smiles>C=Cc1ccc(CCl)cc1</smiles>

22<smiles>O=S(=O)(S)c1ccccc1</smiles>

5

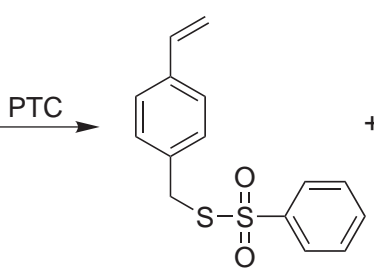

26<smiles>C=Cc1ccc(CO)cc1</smiles>

27

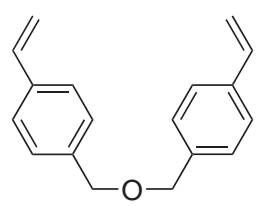

28

Schema 8.1 Haupt- und Nebenprodukte in der ViBSuT-Synthese. 


\subsection{Einfluss auf die Copolymerisation}

Als Comonomer wurde Styrol gewählt, da diese Kombination aufgrund der Ähnlichkeit der Monomere den größten Erfolg verspricht. Die Polymerisationsmischung hatte eine Ausgangskonzentration von 3,09 mol-\% ViBSuT in Bezug auf Styrol (unter Vernachlässigung der Verunreinigungen).

Tabelle 8.1 Ergebnisse der Styrol-ViBSuT-Copolymerisation. ${ }^{a}$ In Styroläquivalenten.

\begin{tabular}{lccccccc}
\hline$\#$ & $\begin{array}{c}{[\mathrm{ViBSuT}] /} \\
\mathrm{mol}-\%\end{array}$ & $\begin{array}{c}{[\mathrm{AIBN}] /} \\
\mathrm{mol-} \%\end{array}$ & $\begin{array}{c}\mathrm{T} / \\
{ }^{\circ} \mathrm{C}\end{array}$ & $\begin{array}{c}\text { t/ } \\
\mathrm{h}\end{array}$ & $\begin{array}{c}\bar{M}_{\mathrm{n}}^{a} / \\
(\mathrm{g} / \mathrm{mol})\end{array}$ & $\begin{array}{c}\mathrm{U} / \\
\%\end{array}$ \\
\hline $\mathrm{M} 21$ & 3,09 & 0,05 & 60 & 6 & $2,35 \cdot 10^{3}$ & 2,74 & 4,4 \\
\hline
\end{tabular}

Um den Umsatz zu bestimmen wurde das Polymer in einer geringen Menge THF gelöst, in einem Überschuss kaltem Methanol gefällt, abfiltriert und im Vakuum getrocknet. Aus dem Schwefelgehalt, der mittels Elementaranalyse bestimmt wurde, lässt sich direkt die ViBSuT-Konzentration im Polymer bestimmen, da die Verunreinigungen schwefelfrei sind (Tabelle 8.2). Die drei durchgeführten Analysen ergaben einen Schwefelgehalt von 10,46-11,23\%, was einem ViBSuT-Gehalt von 24,4 - 27,1\% entspricht.

Tabelle 8.2 Ergebnisse der Elementaranalyse des Styrol-ViBSuT-Copolymers M21.

\begin{tabular}{lcccc}
\hline$\#$ & $\mathrm{C} / \%$ & $\mathrm{H} / \%$ & $\mathrm{~S} / \%$ & ViBSuT-Gehalt/mol-\% \\
\hline Styrol & 92,26 & 7,74 & & \\
ViBSuT & 62,04 & 4,86 & 22,08 & \\
\hline M21-I & 78,13 & 6,33 & 11,23 & 27,1 \\
M21-II & 78,53 & 6,41 & 10,46 & 24,4 \\
M21-III & 78,28 & 6,46 & 10,56 & 24,7 \\
\hline
\end{tabular}

Das Vorhandensein großer Restmengen des Comonomers ViBSuT kann aus verschiedenen Gründen ausgeschlossen werden. Zum einen beträgt die Masse des getrockneten Polymers 658 mg, während die Rohproduktmischung 1,2197 g ViBSuT enthielt. Somit muss bei der Aufarbeitung durch Fällung zumindest ein Teil entfernt worden sein. Zum anderen zeigt die Molmassenverteilung Abbildung 8.1 keinen deutlichen Peak im niedermolekularen Bereich, wie er z. B. aus RAFT-Polymerisationen mit unverbrauchtem 
RAFT-Agens bekannt ist. Daher ist davon auszugehen, dass der Schwefelgehalt der Polymerprobe aus eingebauten Monomereinheiten besteht.

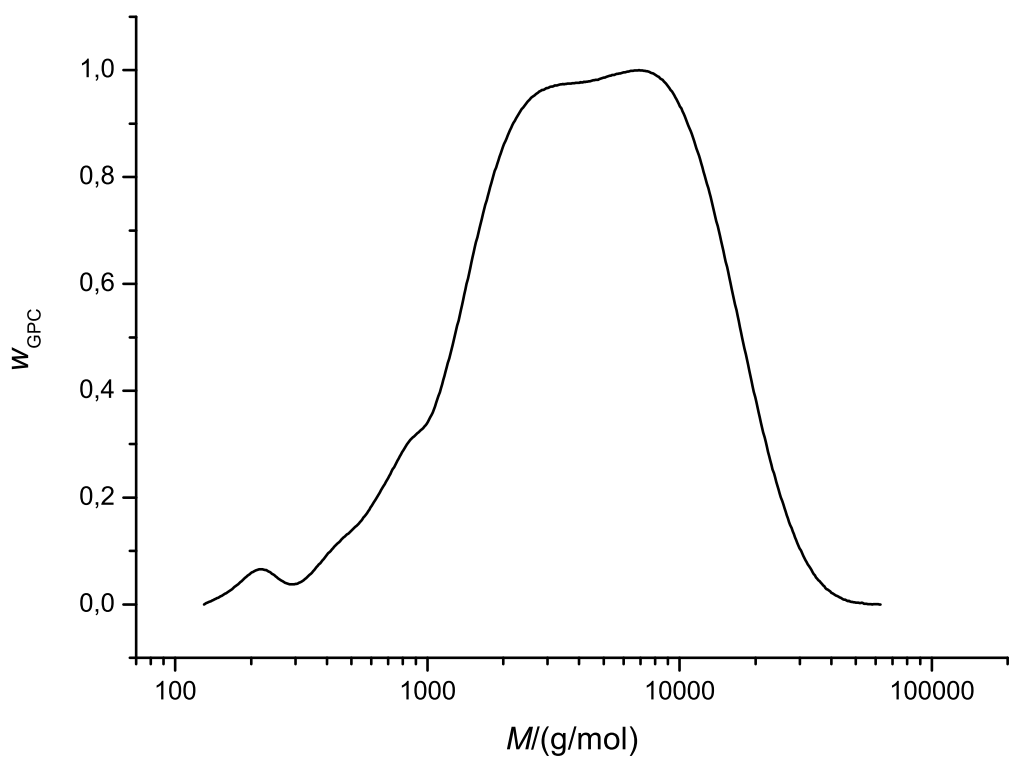

Abbildung 8.1 Molmassenverteilung der Styrol-ViBSuT-Copolymerisation M21 (siehe Tabelle 8.1).

Die Molmassenverteilung zeigt deutlich, dass es bereits während der relativ kurzen Polymerisationszeit von nur $6 \mathrm{~h}$ zu Vernetzungsreaktionen kommt. Die Dispersität liegt mit einem Wert von 2,74 deutlich über der für eine freie radikalische Styrolpolymerisation zu erwartenden Dispersität. Die Struktur der Molmassenverteilung zeigt eine nahezu konstante Intensität für Kettenlängen zwischen 2000 und $10000 \mathrm{~g} / \mathrm{mol}$. Eine mögliche Ursache ist die Anwesenheit kleiner Mengen des Dimers 28 (0,3 mol-\% bezogen auf die Gesamtmischung), welches ein bifunktionales Monomer ist. Werden beide Vinylreste einpolymerisiert, können sich daher Polymere bilden, deren Molmasse deutlich größer ist, da sie zwei Polymerketten miteinander verbinden können und insgesamt zu Vernetzungen führen. Darüber hinaus ist es möglich, dass die vulkanisationsaktive Gruppe des funktionalisierten Monomers ViBSuT bereits bei der radikalischen Polymerisation angegriffen und gespalten wird. Somit bilden sich auch hier zwei Verknüfungspunkte und es kann zu einer Vernetzung zweier Polymerketten kommen.

Da die GPC die Molmassen der Polymermischung nach ihren hydrodynamischen Volumina separiert, hat die gemittelte Molmasse nur eine eingeschränkte Bedeutung. Es wurde für die Messung auf eine Styrol-Kalibrierung zurückgegriffen, die für ein Styrol- 
ViBSuT-Copolymer nicht ideal ist, da dieses Copolymer unter Umständen ein anderes Knäuelverhalten in Lösung und ein anderes hydrodynamisches Volumen aufweist und dadurch eine verfälschte Molmasse zugeordnet bekommt. Darüber hinaus ändert eine Vernetzung das Knäuelverhalten und somit die detektierte Molmasse in Abweichung zur wahren Molmasse.

\subsection{Fazit}

Wie zu erwarten war, führt das bifunktionelle Monomer, welches sich als Nebenprodukt in der ViBSuT-Synthese bildet, zu Vernetzungen. Darüber hinaus ist es möglich, dass die funktionelle Gruppe bereits während der Polymerisation vernetzt und nicht erst in der Vulkanisation. Um dies beurteilen zu können, ist eine Copolymerisation mit einer chemisch reinen ViBSuT-Probe notwendig. Die Verwendung eines Produktgemischs mit kleineren Anteilen binfunktionellem Monomer kann dafür erste Anhaltspunkte liefern.

Eine Vernetzung bereits während der Polymerisation ist nicht per se ein Ausschlusskriterium, sofern genug Netzknotenpunkte für eine Vernetzung während der Vulkanisation übrig bleiben und so eine Vernetzung mit den anderen Komponenten (z. B. Naturkautschuk) gewährleistet ist. Eine Verringerung des Comonomergehalts kann ebenfalls helfen, Vernetzungen während der Polymerisation zu verringern, führt aber gleichzeitig zu weniger Vernetzungsmöglichkeiten in der Vulkanisation. 


\section{Schaltbare RAFT-Agenzien}

Ein weiteres Ziel dieser Arbeit bestand darin, mit Ethen ein zusätzliches, unpolares Monomer zu benutzen. Da dies einen umfangreicheren experimentellen Aufbau bedingt, weil Ethen genauso wie Butadien ein Gas ist, wurden erste Experimente mit Vinylacetat durchgeführt. Die Ergebnisse dieses Modellsystems lassen sich dann gut auf Ethen übertragen, da beide in ihrem Polymerisationsverhalten sehr ähnlich sind. Des Weiteren haben sie gemeinsam, dass sie sich nicht mit Butadien oder Styrol radikalisch zu einem statistischen Copolymer polymerisieren lassen, da die Anlagerung des jeweils anderen Monomers an ein Monomerradikal nicht begünstigt ist.

Die Polymerisation von Butadien oder Styrol mit einem protonierten schaltbaren RAFT-Agens liefert ein makromolekulares RAFT-Agens (Makro-RAFT-Agens), welches anschließend deprotoniert in einer Vinylacetat-Polymerisation zur Herstellung eines Blockcopolymers verwendet werden kann.

Es wurde dabei auf die bereits bekannten schaltbaren RAFT-Agenzien zurückgegriffen. ${ }^{[49,50]}$ Während die Z-Gruppe aufgrund der protonierbaren Einheit wenig Spielraum bietet, besteht die Möglichkeit, die Abgangsgruppe zu modifizieren und an die Bedürfnisse des eigenen Systems anzupassen. So könnte z. B. eine potenziell vulkanisierbare Gruppe in die Abgangsgruppe integriert werden und so ein Einbau der Kettenenden in ein Netzwerk begünstigt werden. 


\subsection{Reagenzien}

Die Synthesen der schaltbaren RAFT-Agenzien sind in Veröffentlichungen und Patenten publiziert, ${ }^{[44,49,50]}$ so dass auch Derivate leicht zugänglich sind. Bei den RAFTAgenzien 29 und 30 wird die Abgangsgruppe R in einer Substitutionsreaktion durch die entsprechende Bromverbindung eingeführt, bei 31 und 32 wird zuerst das Dithiuramdisulfid 33 hergestellt, welches mit den Azoinitiatoren AIBN bzw. V70 (2,2'Azobis-(4-methoxy-2,4-dimethyl-valeronitril)) umgesetzt wird, um das gewünschte RAFT-Agens (31 bzw. 32) zu erhalten. In einem Patent ${ }^{[127]}$ wurden weitere Derivate dieser RAFT-Agenzien vorgestellt. Da die schaltbaren RAFT-Agenzien eine zunehmende Bedeutung erlangen, sind die Verbindungen 29, 30 und 31 sowie das Dimer 33 seit kurzem kommerziell erhältlich.

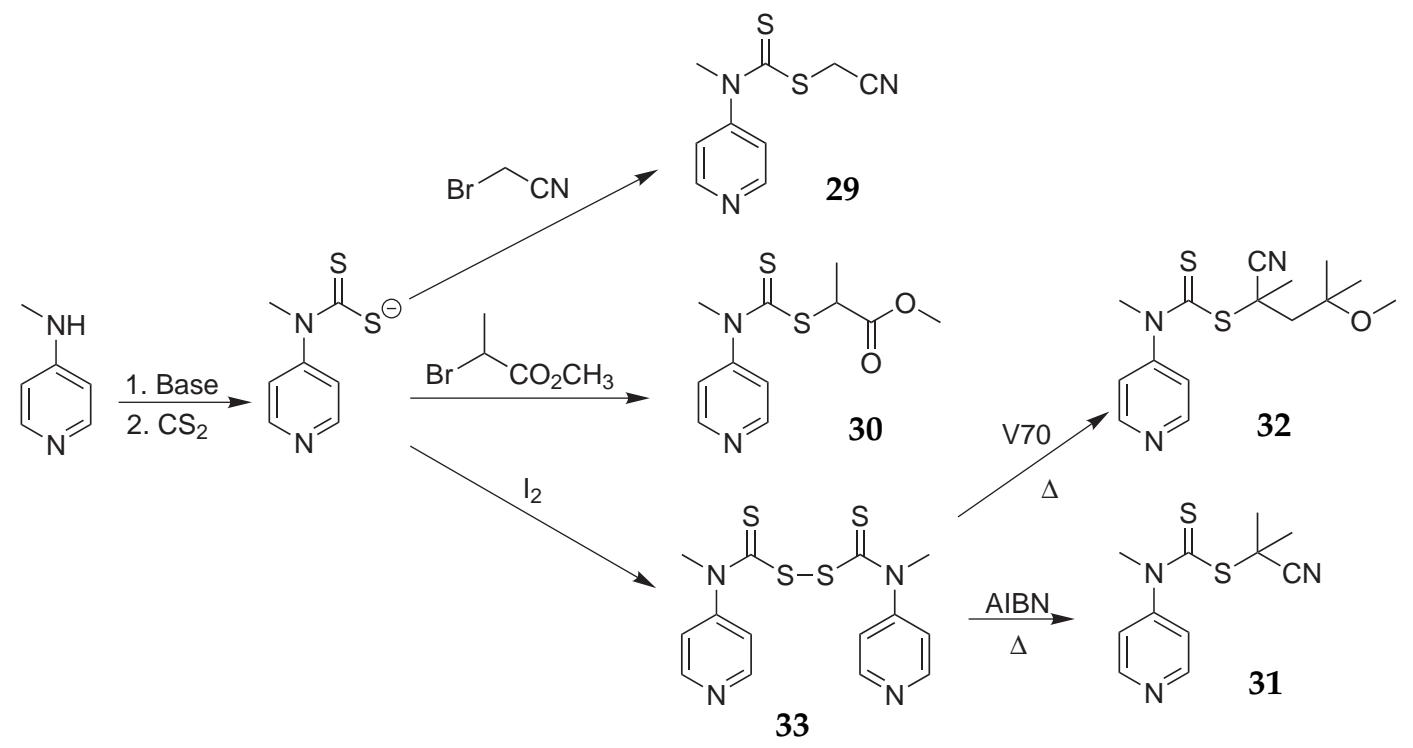

Schema 9.1 Synthese der schaltbaren RAFT-Agenzien. ${ }^{[4]}$

Im Rahmen dieser Arbeit wurden mit den RAFT-Agenzien 30 und 31 Poly(styrol)-blockpoly(vinylacetat) und Poly(butadien)-block-poly(vinylacetat) hergestellt. Da schaltbare RAFT-Agenzien ein relativ neues Thema sind, gibt es bislang wenig literaturbekannte Polymerisationen. Blockcopolymere mit Butadien sind bislang generell unbekannt. Außerdem sind keine Ergebnisse einer Styrolhomopolymerisation oder einer StyrolVinylacetat-Blockcopolymerisation mit dem RAFT-Agens 31 veröffentlicht. 


\subsection{MAM-Polymerisation}

Da terminale LAM-Einheiten schlechtere Radikalabgangsgruppen als terminale MAMEinheiten sind, wurde bei der Diblock-Polymerisation mit der Synthese des MAMBlockes begonnen. Als MAM dienten Styrol und Butadien, als LAM Vinylacetat, wobei Styrol und Vinylacetat als Modellsystem fungieren.

\subsubsection{Styrol-Polymerisation}

Die Synthese des Styrol-Blockes erfolgte analog zu der Vorschrift von Benaglia et al. ${ }^{[44,49,50]}$ mit den RAFT-Agenzien 30 und 31. Dies diente dazu, die Reproduzierbarkeit der Literaturwerte zu überprüfen und die Verwendbarkeit des RAFT-Agens mit der tertiären Abgangsgruppe in einer Styrol-Polymerisation zu untersuchen, da dies bislang nicht literaturbekannt ist. Zur Protonierung des RAFT-Agens wurde $p a-$ $r a$-Toluolsulfonsäure ( $p$ - $\mathrm{TsOH})$ bzw. Trifluormethansulfonsäure ( $\mathrm{TfOH})$ verwendet. Die Löslichkeit von para-Toluolsulfonsäure in Styrol ist bei Raumtemperatur unzureichend und somit ist aufgrund der äquimolaren Einwaage keine vollständige Protonierung gewährleistet. Um dies zu beheben, wurde u. a. eine Polymerisation mit Acetonitril als Lösungsmittel durchgeführt (S04) oder die Reaktionsmischung gerüht (S06). Bei der Polymerisationstemperatur von $90^{\circ} \mathrm{C}$ waren jedoch immer alle Komponenten gelöst und das Rühren hat somit lediglich eine bessere Durchmischung gewährleistet.

Die Polymerisationsmischungen wurden entgast, sofern nicht anders angegeben. Die RAFT-Agens- sowie Initiator-Konzentration wurde dabei nicht variiert, sondern an die Literaturwerte angepasst und beibehalten. Die Säure wurde äquimolar zum RAFTAgens eingesetzt. Das Ziel, kurze Blöcke $\left(\bar{M}_{\mathrm{n}} \geq 2000 \mathrm{~g} / \mathrm{mol}\right) \mathrm{zu}$ synthetisieren, wurde weiterhin verfolgt und daher die Polymerisationszeit auf wenige Stunden beschränkt. Die Ergebnisse von Benaglia et al. mit 30 konnten dabei gut reproduziert werden. S01A$\mathrm{D}$ weisen auf eine erfolgreiche RAFT-Polymerisation hin, da die mittlere Molmasse mit steigendem Styrol-Umsatz bei gleichbleibend kleinen Dispersitäten nach 2, 3 bzw. $4 \mathrm{~h}$ Polymerisationszeit zunimmt. Die vergleichsweise hohe Dispersität von 1,62 nach $1 \mathrm{~h}$ ist darauf zurückzuführen, dass das Vorgleichgewicht noch nicht vollständig durchlaufen wurde und molekulares RAFT-Agens zu einer erhöhten Uneinheitlichkeit beiträgt (siehe Abbildung 9.1). Unterschiedliche Polymerisationsansätze mit RAFT-Agens 31 haben hingegen zu deutlich abweichenden Ergebnissen geführt. Die Polymerisation in Lösung (S04) kann nicht direkt mit den Polymerisationen in Substanz (S05-S07) 
verglichen werden. Dennoch variieren auch bei den Polymerisationen S05-S07 die Dispersitäten zwischen 1,08 und 1,47, der Monomerumsatz zwischen 18,0 und 24,9\% und die mittlere Molmasse zwischen 1,97 $10^{3}$ und 5,46 $10^{3} \mathrm{~g} / \mathrm{mol}$.

Tabelle 9.1 Ergebnisse der Styrol-Polymerisationen mit $70 \mathrm{mmol} / \mathrm{L}$ schaltbarem RAFT-Agens und $10 \mathrm{mmol} / \mathrm{L}$ ACCN als Initiator bei $90^{\circ} \mathrm{C}$ in Substanz im Vergleich mit Literaturdaten. ${ }^{a}$ Lösung mit $\mathrm{N}_{2}$ für 10 min gespült. ${ }^{b}$ Störung durch ionische Polymerisation. ${ }^{c}$ Acetonitrillösung (1:1). ${ }^{d}$ Inhomogen. ${ }^{e}$ Lösung während der Polymerisation gerührt.

\begin{tabular}{|c|c|c|c|c|c|c|}
\hline \# & RAFT & Säure & $t / \mathrm{h}$ & $\bar{M}_{\mathrm{n}} / \mathrm{g} / \mathrm{mol}$ & $Đ$ & $U / \%$ \\
\hline $\mathrm{S}_{01 \mathrm{~A}^{a}}$ & $30-\mathrm{H}^{+}$ & $p$-TsOH & 1 & $0,71 \cdot 10^{3}$ & 1,62 & 9,9 \\
\hline $\mathrm{S} 01 \mathrm{~B}^{a}$ & $30-\mathrm{H}^{+}$ & $p$-TsOH & 2 & $1,52 \cdot 10^{3}$ & 1,22 & 17,5 \\
\hline S01C ${ }^{a}$ & $30-\mathrm{H}^{+}$ & $p$-TsOH & 3 & $2,31 \cdot 10^{3}$ & 1,19 & 26,7 \\
\hline $\mathrm{S}_{01 \mathrm{D}^{a}}$ & $30-\mathrm{H}^{+}$ & $p$-TsOH & 4 & $2,95 \cdot 10^{3}$ & 1,13 & 33,2 \\
\hline S02 & $30-\mathrm{H}^{+}$ & $p$-TsOH & 2 & $2,76 \cdot 10^{3}$ & 1,19 & 24,7 \\
\hline $\mathrm{S}_{0} 3^{b}$ & $30-\mathrm{H}^{+}$ & $\mathrm{TfOH}$ & 2 & $5,90 \cdot 10^{3}$ & 2,16 & 39,6 \\
\hline $\mathrm{S} 04^{c}$ & $31-\mathrm{H}^{+}$ & $p$-TsOH & 2 & $1,53 \cdot 10^{3}$ & 1,18 & 7,6 \\
\hline $\mathrm{S} 05^{d}$ & $31-\mathrm{H}^{+}$ & $p$-TsOH & 2 & $5,46 \cdot 10^{3}$ & 1,40 & 21,1 \\
\hline $\mathrm{S} 06^{a, e}$ & $31-\mathrm{H}^{+}$ & $p$-TsOH & 2 & $1,97 \cdot 10^{3}$ & 1,47 & 18,0 \\
\hline $\mathrm{S} 07^{a}$ & $31-\mathrm{H}^{+}$ & $\mathrm{TfOH}$ & 2 & $3,49 \cdot 10^{3}$ & 1,08 & 24,9 \\
\hline $\mathrm{S} 08^{[50]}$ & $30-\mathrm{H}^{+}$ & $p-\mathrm{TsOH}$ & 2 & $1,8 \cdot 10^{3}$ & 1,22 & 18 \\
\hline S09 [50] & $30-\mathrm{H}^{+}$ & $p$-TsOH & 2 & $1,8 \cdot 10^{3}$ & 1,33 & 20 \\
\hline$S 10^{[49]}$ & $30-\mathrm{H}^{+}$ & $\mathrm{TfOH}$ & 12 & $10,5 \cdot 10^{3}$ & 1,06 & 82 \\
\hline
\end{tabular}

\subsubsection{Butadien-Polymerisation}

Neben Styrol wurden auch Butadien-Polymerisationen mit schaltbaren RAFTAgenzien durchgeführt. Nach Deprotonierung könnten diese in VinylacetatPolymerisationen eingesetzt und so erstmals Poly(butadien)-block-poly(vinylacetat) mittels RAFT hergestellt werden. Die Zusammensetzungen der Polymerisationsansätze wurden in Anlehnung an die Styrol-Polymerisationen berechnet. Da die Polymerisation aufgrund des Aggregatzustandes von Butadien nicht in Substanz durchgeführt werden kann, wurde ein Lösungsmittel (für gewöhnlich Toluol) zugegeben. Die Löslichkeit von para-Toluolsulfonsäure in Toluol ist sehr gering. Dennoch wurde in Toluol 


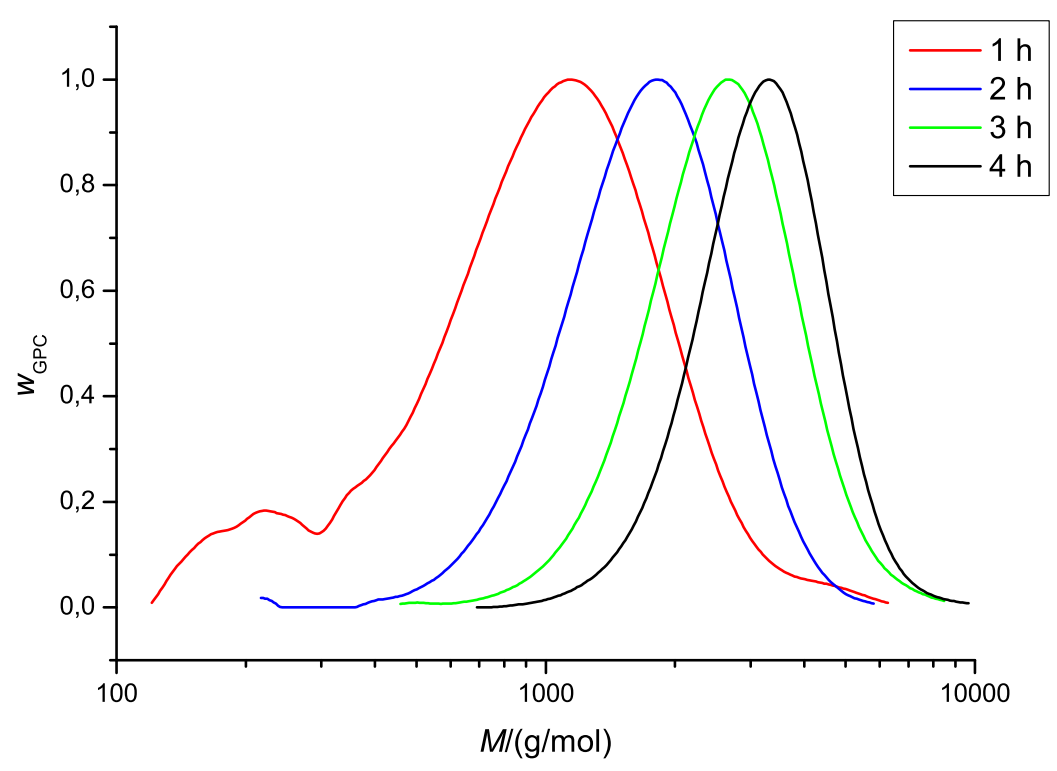

Abbildung 9.1 Molmassenverteilungen der Styrol-Polymerisationen S01A-D mit dem schaltbaren RAFT-Agens 30 (siehe Tabelle 9.1).

gearbeitet, da die Vorteile überwiegen. Toluol löst das Monomer Butadien sehr gut und hat einen vergleichsweise hohen Siedepunkt von $111^{\circ} \mathrm{C}$. Wird die Polymerisation bei $80^{\circ} \mathrm{C}$ durchgeführt, ist Toluol noch flüssig. Auf der anderen Seite ist der Dampfdruck hoch genug, dass es auch in großen Mengen gut von der Polymerprobe abdampft. Die Ergebnisse der Polymerisationen sind tabellarisch in Tabelle 9.2 dargestellt sowie grafisch in Abbildung 9.2.

Es ist deutlich ersichtlich, dass trotz der langen Polymerisationszeit von $48 \mathrm{~h}$ keine hohen Umsätze erreicht werden konnten, die Polymerisation somit sehr langsam verläuft. Bei sämtlichen Proben, die nach der Deprotonierung des RAFT-Agens analysiert wurden, ist ein Peak im niedermolekularen Bereich sichtbar. Die Probe, die von der Polymerisation S13 stammt und sowohl vor als auch nach der Deprotonierung gemessen wurde, weist nur im deprotonierten Zustand diesen Peak auf. Dies deutet darauf hin, dass unter basischen Bedingungen (bei der Filtration durch Natriumcarbonat) eine Bindung gespalten wurde (möglicherweise die Dithioeinheit des RAFT-Agenzes). Eine Fällung des Polymers zur Abtrennung des niedermolekularen Anteils ist bei den geringen Molmassen der Polymere nicht möglich. Die Polymere der Polymerisationen S11 und S12 wurden aufgrund ihrer extrem kurzen Ketten und den niedermolekularen Verunreinigungen nicht weiter verwendet. An das Polymer S13 wurde in einer Vinylacetat- 


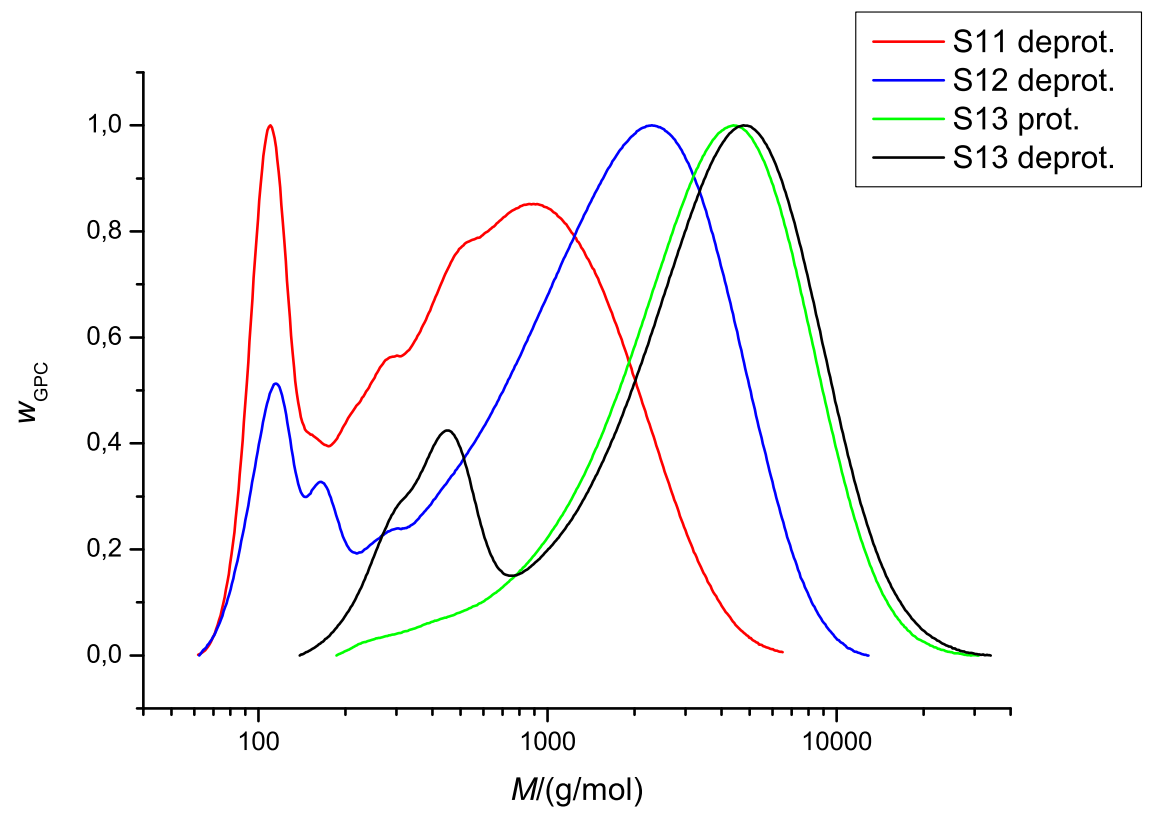

Abbildung 9.2 Molmassenverteilungen der Butadien-Polymerisationen S11-S13 mit den schaltbaren RAFT-Agenzien 30 und 31 (siehe Tabelle 9.2).

Polymerisation ein Vinylacetat-Block polymerisiert, die Ergebnisse werden in Kapitel 9.3.2 vorgestellt.

Tabelle 9.2 Ergebnisse der Butadien-Polymerisationen in Toluol mit schaltbaren RAFT-Agenzien, protoniert mit para-Toluolsulfonsäure, bei $80^{\circ} \mathrm{C}$ nach $48 \mathrm{~h}$.

\begin{tabular}{|c|c|c|c|c|c|c|c|c|}
\hline \# & $\begin{array}{l}{[\mathrm{BD}] /} \\
\text { mol-\% }\end{array}$ & RAFT & $\begin{array}{c}\text { [RAFT]/ } \\
\text { mol-\% }\end{array}$ & $\begin{array}{c}{[\mathrm{ACCN}] /} \\
\text { mol-\% }\end{array}$ & $\begin{array}{l}{[\mathrm{Tol}] /} \\
\text { mol-\% }\end{array}$ & $\begin{array}{c}\bar{M}_{\mathrm{n}} / \\
(\mathrm{g} / \mathrm{mol})\end{array}$ & $Ð$ & $U / \%$ \\
\hline S11 & 34,4 & $30-\mathrm{H}^{+}$ & 0,184 & 0,037 & 65,2 & $0,31 \cdot 10^{3}$ & 2,66 & 1,3 \\
\hline S12 & 35,3 & $30-\mathrm{H}^{+}$ & 0,204 & 0,072 & 64,2 & $0,53 \cdot 10^{3}$ & 3,65 & 11,1 \\
\hline $\mathrm{S} 13$ & 35,4 & $31-\mathrm{H}^{+}$ & 0,078 & 0,016 & 64,4 & $1,48 \cdot 10^{3}$ & 2,39 & 6,1 \\
\hline
\end{tabular}




\subsection{LAM-Polymerisation}

Zur Synthese des Vinylacetatblockes wurde ein Makro-RAFT-Agens verwendet, welches vorher deprotoniert wurde. Dies geschah durch Filtration des in Dichlormethan gelösten Polymers durch Natriumcarbonat. Bei Polystyrol wechselte die Farbe der Polymerlösung dabei von gelb zu farblos, bei Polybutadien von orange zu blassgelb. Dieser Farbumschwung wird durch die geänderte elektronische Struktur des RAFT-Agens hervorgerufen, da das Proton Elektronendichte aus dem Phenylring und der SchwefelKohlenstoff-Doppelbindung abzieht.

Es wurden Polystyrol-Makro-RAFT-Agenzien 34 und 35 mit einer mittleren Molmasse von ca. $3000 \mathrm{~g} / \mathrm{mol}$ ausgewählt, was im Durchschnitt rund 25 Styrol-Einheiten entspricht $(M=104,15 \mathrm{~g} / \mathrm{mol})$. Bei dem Butadien-Makro-RAFT-Agens 36 mit einer Molmasse von ca. $1500 \mathrm{~g} / \mathrm{mol}$ sind rund 23 Butadien-Einheiten einpolymerisiert. Die RAFT-Agenzien haben somit einen ähnlichen Polymerisationsgrad.
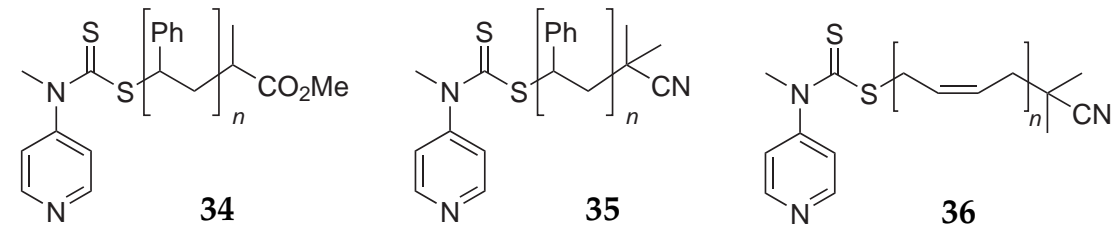

Schema 9.2 Die verwendeten Makro-RAFT-Agenzien 34 (30-PSty), 35 (31-PSty) und 36 (31-PBD).

\subsubsection{Vinylacetat-Polymerisation mit Styrol-Makro-RAFT-Agens}

Die Vinylacetat-Polymerisationen mit Makro-RAFT-Agens 34 wird zu Beginn retardiert, wie in Abbildung 9.3 ersichtlich. Die Proben, die einer Polymerisationszeit von 1 bis $4 \mathrm{~h}$ entsprechen, unterscheiden sich kaum von dem eingesetzten Makro-RAFTAgens, schwarz gestrichelt dargestellt. Es bildet sich auf der höhermolekularen Seite eine Schulter, die von Poly(styrol)-block-poly(vinylacetat) stammt. Erst nach einer Polymerisationszeit von $6 \mathrm{~h}$ bewirkt diese eine deutliche Verschiebung der Molmasse. Nach $24 \mathrm{~h}$ ist diese Schulter nicht mehr sichtbar, alle RAFT-Moleküle haben einen Polyvinylacetatblock eingebaut und das RAFT-Vorgleichgewicht wurde vollständig durchlaufen. Der Grund für die Retardierung liegt in der mangelnden Reaktivität von Polystyrol- 


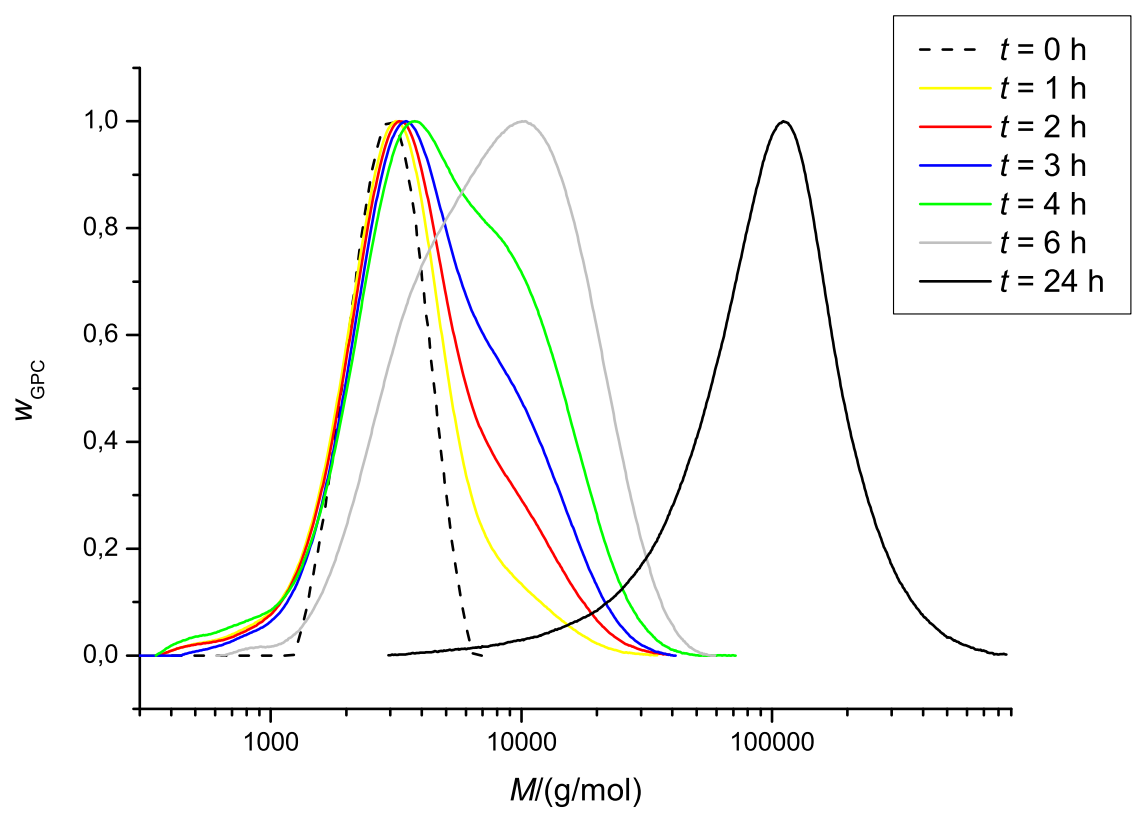

Abbildung 9.3 Molmassenverteilungen der Vinylacetat-Polymerisationen S14A-F mit dem schaltbaren Makro-RAFT-Agens S02 (gestrichelt dargestellt) in StyrolÄquivalenten.

Radikalen gegenüber Vinylacetat.

Die frühzeitig hohen Umsätze von Benaglia et al. ${ }^{[50]}$ ließen sich nicht reproduzieren, da die Polymerisationen einer starken Anfangsretardierung unterworfen sind. Die Gründe dafür bleiben aufgrund der vergleichbaren Reaktionsführung und Zusammensetzung der Polymerisationsmischung offen. Nichtsdestotrotz zeigen beide Polymerisationen, dass es möglich ist, an den bestehenden Polystyrolblock einen Polyvinylacetatblock mittels RAFT zu polymerisieren.

Die beiden Makro-RAFT-Agenzien 34 und 35 unterscheiden sich in der Abgangsgruppe $\mathrm{R}$ des ursprünglichen RAFT-Agens sowie geringfügig in ihrer mittleren Molmasse (2760 g/mol bzw. $3490 \mathrm{~g} / \mathrm{mol})$ und Dispersität (1,19 bzw. 1,08). Da die Abgangsgruppe bei den Makro-RAFT-Agenzien durch mehrere Monomereinheiten von der aktiven RAFT-Einheit, dem Dithioester, getrennt ist, sollte der Unterschied marginal sein. Sobald eine Monomereinheit an das Radikal R. addiert wurde, ist die Propagationsgeschwindigkeit des Makroradikals sowie die Fragmentierungsgeschwindigkeit des Intermediats gleich.

Mit beiden RAFT-Agenzien lässt sich beobachten, dass die Molmassenverteilungen 
Tabelle 9.3 Ergebnisse der Vinylacetat-Polymerisationen mit schaltbarem MakroRAFT-Agens aus der Polymerisation $\mathrm{S} 02$ bei $70{ }^{\circ} \mathrm{C}$ in Substanz im Vergleich mit Literaturdaten.

\begin{tabular}{lcccccccc}
\hline$\#$ & RAFT & $10^{-2} M$ & Initiator & $10^{-3} M$ & $t / \mathrm{h}$ & $\bar{M}_{\mathrm{n}} / \mathrm{g} / \mathrm{mol}$ & $\oplus$ & $U / \%$ \\
\hline S14A & 34 & 1,0 & AIBN & 5,66 & 1 & $2,72 \cdot 10^{3}$ & 1,45 & 3,7 \\
S14B & 34 & 1,0 & AIBN & 5,66 & 2 & $3,06 \cdot 10^{3}$ & 1,59 & 4,3 \\
S14C & 34 & 1,0 & AIBN & 5,66 & 3 & $3,55 \cdot 10^{3}$ & 1,63 & 4,8 \\
S14D & 34 & 1,0 & AIBN & 5,66 & 4 & $3,72 \cdot 10^{3}$ & 1,84 & 5,7 \\
S14E & 34 & 1,0 & AIBN & 5,66 & 6 & $5,90 \cdot 10^{3}$ & 1,71 & 8,5 \\
S14F & 34 & 1,0 & AIBN & 5,66 & 24 & $67,13 \cdot 10^{3}$ & 1,75 & 94,1 \\
S15A $^{[50]}$ & 34 & 1,08 & AIBN & 5,7 & 2 & $5,40 \cdot 10^{3}$ & 2,16 & 4,6 \\
S15B $^{[50]}$ & 34 & 1,08 & AIBN & 5,7 & 3 & $18,2 \cdot 10^{3}$ & 2,00 & 23 \\
S15C $^{[50]}$ & 34 & 1,08 & AIBN & 5,7 & 4 & $50,2 \cdot 10^{3}$ & 1,83 & 67 \\
S15D $^{[50]}$ & 34 & 1,08 & AIBN & 5,7 & 8 & $73,6 \cdot 10^{3}$ & 1,95 & 100 \\
S15E $^{[50]}$ & 34 & 1,08 & AIBN & 5,7 & 15 & $73,7 \cdot 10^{3}$ & 2,13 & 100 \\
\hline
\end{tabular}

der Polymere eine Schulter im höhermolekularen Bereich aufweisen, während sich ein Großteil der Ketten noch im Vorgleichgewicht befindet. Erst ab 6 h Polymerisationszeit ist eine deutliche Änderung in der Molmassenverteilung ersichtlich und nach $24 \mathrm{~h}$ ist das Vorgleichgewicht durchlaufen.

Tabelle 9.4 Ergebnisse der Vinylacetat-Polymerisationen mit schaltbarem MakroRAFT-Agens aus Polymerisation $\mathrm{S} 07$ bei $70{ }^{\circ} \mathrm{C}$ in Substanz.

\begin{tabular}{lcccccccc}
\hline$\#$ & RAFT & $10^{-2} M$ & Initiator & $10^{-3} M$ & $t / \mathrm{h}$ & $\bar{M}_{\mathrm{n}} / \mathrm{g} / \mathrm{mol}$ & $Ð$ & $U / \%$ \\
\hline S16A & 31-PS & 1,0 & AIBN & 5,66 & 1 & $3,81 \cdot 10^{3}$ & 1,20 & 4,6 \\
S16B & 37-PS & 1,0 & AIBN & 5,66 & 2 & $4,39 \cdot 10^{3}$ & 1,33 & 5,4 \\
S16C & 37-PS & 1,0 & AIBN & 5,66 & 3 & $5,18 \cdot 10^{3}$ & 1,47 & 6,2 \\
S16D & 37-PS & 1,0 & AIBN & 5,66 & 4 & $6,07 \cdot 10^{3}$ & 1,56 & 7,4 \\
S16E & 37-PS & 1,0 & AIBN & 5,66 & 6 & $9,00 \cdot 10^{3}$ & 1,57 & 10,6 \\
S16F & 37-PS & 1,0 & AIBN & 5,66 & 24 & $28,79 \cdot 10^{3}$ & 1,73 & 43,2 \\
\hline
\end{tabular}




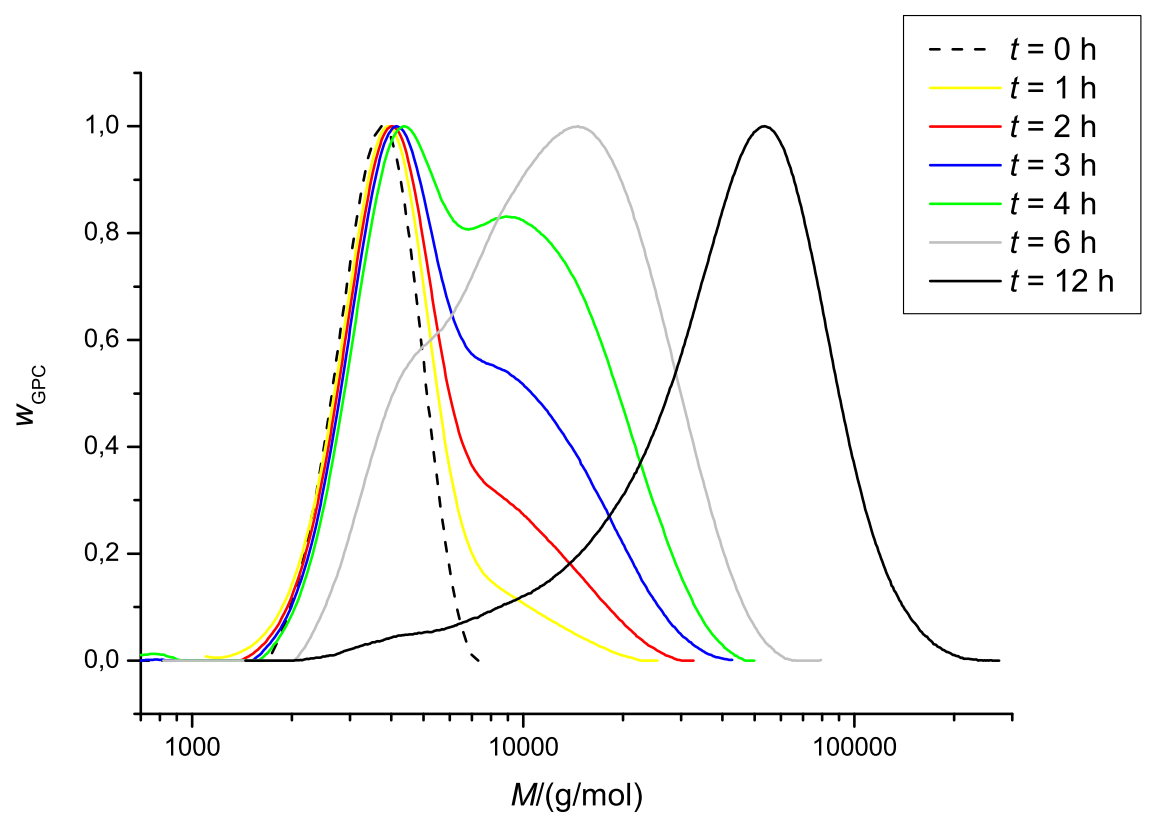

Abbildung 9.4 Molmassenverteilungen der Vinylacetat-Polymerisationen S16A-F mit dem schaltbaren Makro-RAFT-Agens S07 (gestrichelt dargestellt) in StyrolÄquivalenten (siehe Tabelle 9.4).

\subsubsection{Vinylacetat-Polymerisation mit Butadien-Makro-RAFT-Agens}

Das Polybutadien-Makro-RAFT-Agens 36 ist den beiden zuvor diskutierten PolystyrolMakro-RAFT-Agenzien 34 und 35 aufgrund der Kettenlänge des Poymerblockes sehr ähnlich. Es wurde eine vergleichbare Anzahl Monomereinheiten polymerisiert, die aber aufgrund der für Butadien bevorzugten 1,4-Verknüpfung keine Seitengruppen bilden (siehe Schema 9.2). Jede Monomereinheit fügt dem RAFT-Agens eine $C_{4}$-Kette hinzu, wohingegen bei Polystyrol nur eine $\mathrm{C}_{2}$-Kette mit relativ sperriger Seitengruppe, dem Phenylrest, hinzukommt.

Wird Abbildung 9.5 mit den beiden obigen Abbildungen der Vinylacetat-Polymerisationen mit der Polystyrol-Abgangsgruppe verglichen, ist deutlich erkennbar, dass die Butadien-Abgangsgruppe für Vinylacetat-Polymerisationen weitaus besser geeignet ist. Bereits nach $1 \mathrm{~h}$ Polymerisationszeit ist eine Zunahme der Molmasse zu erkennen. Diese Zunahme erfolgt direkt und gleichmäßig bei allen Ketten, es ist keine Schulter sichtbar. 


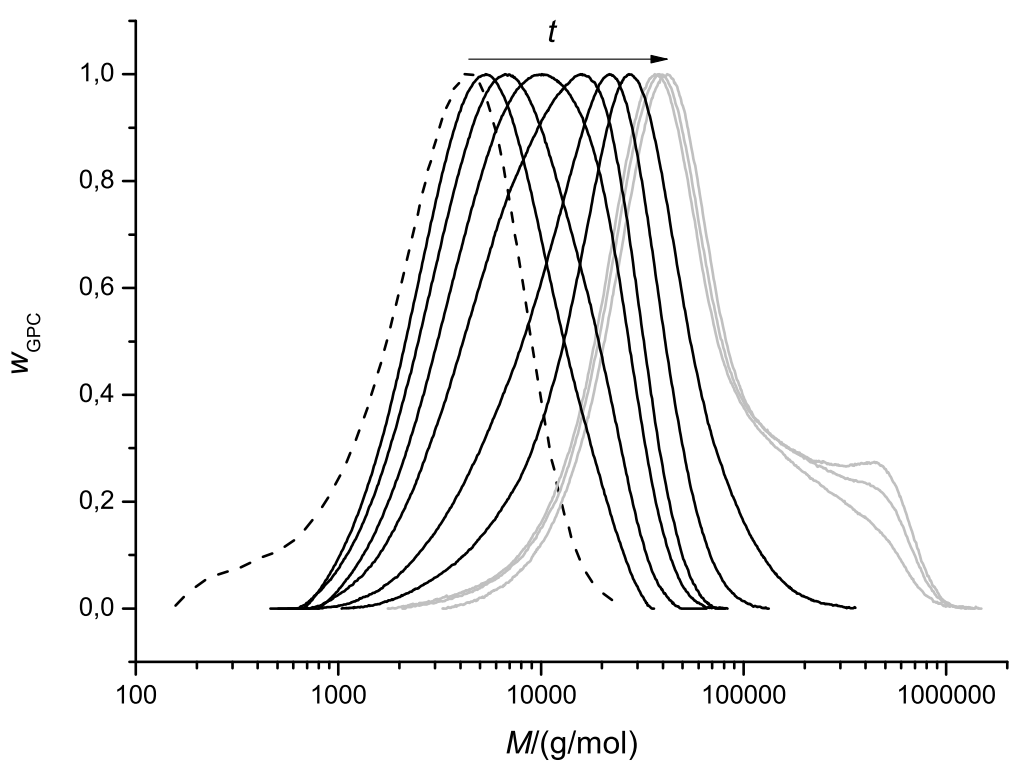

Abbildung 9.5 Molmassenverteilungen der Vinylacetat-Polymerisationen S17A-I mit dem schaltbaren Makro-RAFT-Agens S13 (gestrichelt dargestellt) in ButadienÄquivalenten (siehe Tabelle 9.5). Ab $12 \mathrm{~h}$ Polymerisationszeit kommt es zu Quervernetzungen (grau dargestellt).

Tabelle 9.5 Ergebnisse der Vinylacetat-Polymerisationen mit schaltbarem MakroRAFT-Agens aus der Polymerisation $S 13$ bei $70^{\circ} \mathrm{C}$ in Substanz.

\begin{tabular}{ccccccccc}
\hline$\#$ & RAFT & $10^{-2} M$ & Initiator & $10^{-3} M$ & $t / \mathrm{h}$ & $\bar{M}_{\mathrm{n}} / \mathrm{g} / \mathrm{mol}$ & $Ð$ & $U / \%$ \\
\hline S17A & 37-PBD & 1,0 & AIBN & 5,65 & 1 & $4,03 \cdot 10^{3}$ & 1,67 & 2,6 \\
S17B & 37-PBD & 1,0 & AIBN & 5,65 & 2 & $4,78 \cdot 10^{3}$ & 1,80 & 3,6 \\
S17C & 37-PBD & 1,0 & AIBN & 5,65 & 3 & $6,17 \cdot 10^{3}$ & 1,86 & 4,7 \\
S17D & 37-PBD & 1,0 & AIBN & 5,65 & 4 & $7,40 \cdot 10^{3}$ & 1,86 & 6,5 \\
S17E & 37-PBD & 1,0 & AIBN & 5,65 & 6 & $10,95 \cdot 10^{3}$ & 1,86 & 10,9 \\
S17F & 37-PBD & 1,0 & AIBN & 5,65 & 8 & $17,13 \cdot 10^{3}$ & 1,93 & 18,0 \\
S17G & 37-PBD & 1,0 & AIBN & 5,65 & 12 & $31,08 \cdot 10^{3}$ & 2,82 & 32,4 \\
S17H & 37-PBD & 1,0 & AIBN & 5,65 & 14 & $33,97 \cdot 10^{3}$ & 3,06 & 37,2 \\
S17I & 37-PBD & 1,0 & AIBN & 5,65 & 16 & $38,95 \cdot 10^{3}$ & 2,97 & 41,9 \\
\hline
\end{tabular}


$\mathrm{Ab} 12 \mathrm{~h}$ Polymerisationszeit bildet sich eine Schulter im höhermolekularen Bereich. Dies lässt sich durch die Instabilität von Vinylacetat-Radikalen erklären. Bei hohen Monomerumsätzen, wenn weniger Monomer zur Verfügung steht und eventuell bereits die Viskosität erhöht ist, wird daher unter Umständen ein H-Atom einer anderen Polymerkette abstrahiert, Quervernetzungen sind möglich und dadurch wird die Molmasse drastisch erhöht.

\subsection{Fazit}

Wie gezeigt werden konnte, sind schaltbare RAFT-Agenzien für die Synthese von PolyMAM-block-polyLAM durchaus geeignet. Die unprotonierte Z-Gruppe der RAFTAgenzien eignet sich gut für die Vinylacetat-Polymerisation, die protonierte Variante hat sich sowohl in der Styrol-, als auch in der Butadienpolymerisation bewährt. Eine Anpassung der Abgangsgruppe $\mathrm{R}$ an das Monomer, welches den ersten Block bilden soll, ist sinnvoll und notwendig. Die Anknüpfung des zweiten Blockes bedingt eine Eignung des Polymerblockes als Abgangsgruppe. Dies war bei Styrol nur bedingt gegeben, eine geänderte Versuchsreihenfolge (Vinylacetatblock zuerst) hatte ebenfalls wenig Aussicht auf Erfolg. Dies zeigt den limitierenden Faktor dieser Blockcopolymersynthese. Benaglia et al. ${ }^{[50]}$ wurden ebenfalls auf dieses Problem aufmerksam und haben unterschiedliche Lösungsansätze vorgeschlagen. Dazu gehört die Erhöhung der Initiatorkonzentration sowie die Verwendung von Methylacrylat als zusätzliches Monomer. Ein kurzer Methylacrylatblock zwischen dem Polystyrol- und dem Vinylacetatblock umgeht die Notwendigkeit der Reaktion eines Polystyrolradikals mit einem Vinylacetatmolekül. Darüber hinaus kann die Zugabe einer geringen Menge Methylacrylat zu der Vinylacetatpolymerisation durch die Bildung von Poly(styrol)block-poly(methylacrylat-grad-vinylacetat)-block-polyvinylacetat das Problem beseitigen. Dies ist jedoch nicht zielführend, wenn ausschließlich unpolare Monomere verwendet werden sollen und selbst kleine Mengen Methylacrylat stören.

Bei dem Polybutadien-Makro-RAFT-Agens tritt das Problem der mangelnden Reaktivität der Makroradikale nicht auf. Dennoch hat die Reaktion Optimierungspotential hinsichtlich Monomerumsatz und Molmasse, da bislang nach 48 h nur mäßige Ergebnisse erreicht werden. 


\section{0}

\section{Ausblick}

Im Rahmen dieser Arbeit konnten Butadien enthaltende Copolymere mit gezielt eingebauten vulkanisierbaren Gruppen erfolgreich synthetisiert werden. Darüber hinaus wurden Vorarbeiten für die Synthese von funktionalisiertem Polybutadien geleistet. Zwei Ansätze wurden dabei verfolgt: Die Funktionalisierung von Monomermolekülen durch vulkanisierbare Gruppen, die entlang der Polymerkette eingebaut sind, sowie die Funktionalisierung von RAFT-Agenzien, die zu potenziell vulkanisierbaren Kettenenden führen.

Es konnte gezeigt werden, dass sich das Monomer 4-Vinylbenzylthioacetat (ViBET) quantitativ synthetisieren lässt. Eine wichtige Voraussetzung für den Einsatz in großtechnischen Prozessen ist somit erfüllt. Die Polymerisationen mit ViBET haben gezeigt, dass es sehr wahrscheinlich vulkanisationsaktiv ist, da es bereits während der Polymerisation zu ersten Vernetzungen kommt. Die Propagationsgeschwindigkeit in einer radikalischen Polymerisation mit ViBET ist offensichtlich herabgesetzt. Es ist deshalb wichtig, eine lange Polymerisationszeit und eine hohe Temperatur zu wählen, um Polymere mit einer ausreichend großen Molmasse sowie akzeptable Umsätze zu erhalten. Dank umfangreicher Studien konnte dennoch gezeigt werden, dass das Monomer ViBET für eine Copolymerisation mit Styrol und Butadien durchaus geeignet ist, wobei die Reaktionsbedingungen für optimale Ergebnisse noch überarbeitet 
werden sollten. Das genaue Verhalten in einer Vulkanisation sowie ein Vergleich der Materialeigenschaften von gezielt funktionalisierten und unfunktionalisierten Elastomeren können in einem Chemielabor nicht untersucht werden. Dennoch wurden im Rahmen dieser Arbeit u. a. durch die umfangreichen Experimente zum Verhalten von ViBET auf radikalische Polymerisationen die Grundlagen geschaffen, auf denen im industriellen Umfeld zukünftig weiter aufgebaut werden kann.

Das Monomer 4-Vinylbenzylbenzolthiosulfonat (ViBSuT) konnte nach einer umfangreichen Syntheseoptimierung ebenfalls in sehr guter Reinheit hergestellt werden und steht für weitere Untersuchungen zur Verfügung. Dazu wurden zwei Verfahren entwickelt, die sich primär in der Toxizität der Lösungsmittel (Wasser vs. DMF) unterscheiden. Vor einer großtechnischen Nutzung von ViBSuT sind allerdings noch weitere Experimente notwendig, die u. a. klären, warum sich dieses Monomer in der Polymerisation übermäßig stark anreichert. Sofern sich dieser Effekt in zukünftigen Experimenten reproduzieren lässt, hat dies den Vorteil, dass bereits geringe Mengen des Monomers ausreichen könnten, um die gewünschte Polymerzusammensetzung zu erreichen. Eine gesteuerte Zugabe des Monomers während der Polymerisation könnte, sofern gewünscht, die Bildung von Gradientencopolymeren unterbinden und damit neue Möglichkeiten in der Polymerisation mit diesem Monomer eröffnen.

Neben den Monomeren wurden zwei funktionalisierte RAFT-Agenzien synthetisiert sowie verschiedene funktionalisierte Anker entwickelt, die zur Synthese weiterer RAFT-Agenzien und Monomere zur Verfügung stehen. Während das $\alpha, \omega$ funktionalisierte Trithiocarbonat für eine Styrol- und Butadienpolymerisation nicht geeignet ist, hat sich das monofunktionelle Trithiocarbonat in einer Styrol-Polymerisation bewährt und kann nun zur Funktionalisierung von Polybutadien verwendet werden. Diese beiden Ansätze, die Verwendung von funktionalisierten Monomeren und der Einsatz spezieller RAFT-Agenzien, lassen sich außerdem in einem weiteren Schritt miteinander kombinieren und führen so zu einem $\alpha, \omega$-funktionalisierten Polymer mit gezielt eingebauten funktionellen Gruppen entlang der Polymerkette und somit zu Elastomeren, bei denen die Kettenenden in das Netzwerk integriert werden können und so ein Material mit verbesserten Eigenschaften versprechen.

Darüber hinaus wurde eine Möglichkeit erarbeitet, Blockcopolymere aus Butadien und Vinylacetat in einer kontrollierten radikalischen Polymerisation mit RAFT herzustellen. Die so gewonnenen Erfahrungen lassen sich auf eine Blockcopolymersynthese mit Butadien und Ethylen übertragen und bieten so die einzigartige Möglichkeit diese beiden Monomere in einer RAFT-Polymerisation zu einem Copolymer zu verbinden, was in einer konventionellen radikalischen Polymerisation nicht möglich wäre. 


\section{1}

\section{Experimentalteil}

\subsection{Chemikalien}

Soweit nicht anders vermerkt, wurden kommerziell erhältliche Chemikalien $(A B C R$, Acros Organics, Alfa Aesar, Fisher Scientific, Merck, Roth, Sigma-Aldrich und VWR) mit einer Reinheit von mindestens $95 \%$ ohne weitere Aufreinigung eingesetzt. Für Synthesen wurden Lösungsmittel in pro analysi-Qualität verwendet und für Säulenchromatographie Fassware, die vor der Benutzung destillativ aufgereinigt wurde. Rohprodukte wurden durch Säulenchromatographie an Kieselgel (Partikelgröße 0,06-0,2 mm, Porengröße $60 \AA$, Sigma-Aldrich) gereinigt. Für die ViBSuT-Synthese wurden zwei Pufferlösungen verwendet. Dabei handelt es sich um einen Zitronensäure-NaOH-Puffer ( $\mathrm{pH}(5,00 \pm 0,01)$ bei $\left.20^{\circ} \mathrm{C}\right)$ von Merck und einen Kalium-Natrium-Phosphat-Puffer ( $\mathrm{pH}$ $(7,00 \pm 0,01)$ bei $\left.20^{\circ} \mathrm{C}\right)$ von Fluka. 


\subsection{Monomere}

Styrol (Sigma-Aldrich, $\geq 99 \%$, stabilisiert mit 4-tert-Butylcatechol), Methylacrylat (Sigma-Aldrich, $\geq 99 \%$, stabilisiert mit Hydrochinonmonomethylether) und Vinylacetat (Sigma-Aldrich, $\geq 99 \%$, stabilisiert mit Hydrochinon) wurden vor der Verwendung durch Aluminiumoxid (Sigma-Aldrich, neutral, Brockmann I) filtriert, um Inhibitor und eventuell bereits vorhandenes Polymer abzutrennen. 1,3-Butadien (Air Liquide, $<200$ ppmv $\mathrm{O}_{2},<800$ ppmv $\mathrm{N}_{2},<4000$ ppmv KW) wurde direkt aus der Gasflasche in einen Füllautoklaven überführt und von dort in den Reaktionsautoklaven transferriert. 4-Vinylbenzylchlorid (Fluka bzw. Sigma-Aldrich, 90 \% rein) wurde zuerst bei 4 mbar und $74{ }^{\circ} \mathrm{C}$ destilliert und anschließend durch Aluminiumoxid (s. o.) filtriert, um maximale Reinheit zu erreichen.

\subsection{Initiatoren}

2,2'-Azobis-(iso-butyronitril) und 1,1'-Azobis-(cyclohexancarbonitril) wurden aus Methanol umkristallisiert, im Vakuum getrocknet und bei $-18^{\circ} \mathrm{C}$ gelagert.

\subsection{RAFT-Agenzien}

Die Synthese von S-1-Dodecyl-S'-(iso-butansäure)-trithiocarbonat (DIBTC) 17 erfolgte nach der Vorschrift von Etmimi et al., ${ }^{[101]}$ die auf einer Vorschrift von Lai et al. ${ }^{[100]}$ basiert. Die Synthese von S-1-Dodecyl-S'-(iso-butanazid)-trithiocarbonat (DIAzTC) 18 erfolgte nach der Vorschrift von Gody et al. ${ }^{[99]}$ und die Synthese von Bis(4Vinylbenzyl)trithiocarbonat nach der Vorschrift von Aoyagi und Endo. ${ }^{[94]}$ 


\subsection{Verwendete Geräte}

NMR: ${ }^{1} \mathrm{H}-\mathrm{NMR}-S p e k t r e n$ wurden bei Raumtemperatur mit dem Modell Unity 300 $(300 \mathrm{MHz})$ von Varian oder mit dem Modell Avance III $(300 \mathrm{MHz})$ von Bruker gemessen. ${ }^{13} \mathrm{C}$-NMR-Spektren wurden ebenfalls bei Raumtemperatur auf denselben Geräten bei einer Frequenz von $75 \mathrm{MHz}$ gemessen. Als Lösungsmittel wurden $\mathrm{CDCl}_{3}$, $\mathrm{DMSO}-\mathrm{d}_{6}$ und THF $-\mathrm{d}_{8}$ von Deutero verwendet. Als Referenz diente jeweils das Restprotonensignal des Lösungsmittels. ${ }^{[128,129]}$ Für Polymer-NMRs wurde die Relaxationszeit von $5 \mathrm{~s}$ auf $15 \mathrm{~s}$ verlängert. Zur Auswertung wurde das Programm MestReNova 7.0.3 verwendet. Die Multiplizitäten wurden folgendermaßen abgekürzt: s (Singulett), d (Dublett), t (Triplett), q (Quartett), quin (Quintett), dd (Dublett von Dublett), m (Multiplett).

GPC: Die Molmassenverteilungen der Styrol-, Butadien- und Vinylacetatpolymere sowie der Blockcopolymere wurden mit einem SEC Analysis Systems 1260 Infinity von PSS Agilent gemessen. Das System besteht aus einer PSS Agilent Technologies 1260 Iso Pump G1310B (HPLC-Pumpe), einem Agilent 1260 ALS G1329B Autosampler, einem Agilent 1260 ALS Injektor, einer Vorsäule (PSS SDV, 8 x 50 mm, Partikelgröße $5 \mu \mathrm{m}$ ), drei Trennsäulen (PSS SDV, 8 x 300 mm, Partikelgröße: $5 \mu \mathrm{m}$, Porengröße $105 \AA$, $103 \AA$ and $102 \AA$ ) und den Detektoren. In dieser Arbeit wurden der UV-Detektor PSS Agilent Technologies 1260 VWDVL bei einer Wellenlänge von $310 \mathrm{~nm}$ sowie der RI-Detektor PSS Agilent Technologies 1260 RID benutzt. Als Laufmittel diente THF (HPLC-grade) mit Toluol (> 99,7\%, trocken) als interner Standard (Fließgeschwindigkeit 1,0 mL/min bei $35^{\circ} \mathrm{C}$ ). Das System wurde mit Polystyrol-Standards mit niedriger Polydispersität von PSS kalibriert. Zur Auswertung wurde die Software PSS WinGPC verwendet. Die detektierten Intensitäten wurden auf 1 normiert und, sofern nicht anders angegeben, das Signal des RI-Detektors dargestellt. Für das Methacrlyat-Polymer wurden gemäß dem Prinzip der universellen Kalibrierung die Mark-Houwink-Koeffizienten $K=0,0168 \mathrm{~cm}^{3} / \mathrm{g}$ und $a=0,696^{[130]}$ verwendet.

Massenspektrometrie: Sämtliche ESI-Spektren wurden auf einem Bruker Daltonik ESIToF-MS (micrOTOF) und die EI-Spektren auf einem Jeol (Reflektron) Time-of-Flight-MS in den Laboren der Zentralen Analytik des Institutes für Organische Chemie an der Georg-August-Universität Göttingen gemessen. Bei der ESI wurde bei einer Kapillartemperatur von $180^{\circ} \mathrm{C}$ und einer Spannung von $4,5 \mathrm{kV}$ detektiert. 
Elementaranalyse: Die Elementaranalysen wurden im Analytischen Labor des Institutes für Anorganische Chemie der Georg-August-Universität Göttingen gemessen. Elementaranalysen für Kohlenstoff, Wasserstoff, Stickstoff und Schwefel wurden an einem Elementar Vario EL III Gerät durchgeführt. Der Gehalt der Halogene Chlor und Brom wurde potentiometrisch an einem 636 Titroprocesso von Methrom bestimmt.

ESR: Das ESR-Spektrum wurde auf einem Bruker Elexsys ${ }^{\circledR}$ E 500 series CW (continuous wave) Spektrometer gemessen, welches mit X-Band Frequenz betrieben wird. Zur Temperierung wurde eine 4131VT Einheit (Bruker) verwendet und die Probe mit Stickstoff umspült. Folgende Einstellungen wurden verwendet: Einzelscan: 1342,2 s, Breite: $70 \mathrm{G}$, Zeitkonstanzte: 0,01 ms, Dämpfung: $10 \mathrm{~dB}$, Empfängerzunahme: 70, Mikrowellenleistung: $20 \mathrm{~mW}$, Modulationsamplitude: 3G. Es wurden ESR-Röhrchen aus Quartzglas mit $3 \mathrm{~mm}$ Außen- und $2 \mathrm{~mm}$ Innendurchmesser verwendet.

Mikrowelle: Mikrowellen-unterstützte Synthesen wurden in einem Discover S Mikrowellenreaktor von CEM durchgeführt. Es wurden folgende Bedingungen verwendet: Maximaltemperatur: $80^{\circ} \mathrm{C}$, Leistung: $75 \mathrm{~W}$, Zeit: $15 \mathrm{~min}$, Maximaldruck: 5 bar, Vorrühren: 60 s, Rühren: hoch.

\subsection{Synthesen}

\section{Natriumbenzolthiosulfonat (5)}

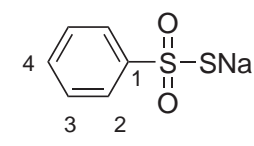

Die Synthese von 5 erfolgte nach der Vorschrift von Sato et al. ${ }^{[2,131]}$ in Pyridin oder n-Butylamin.

${ }^{1}$ H-NMR (300 MHz, DMSO-d ${ }_{6}$ ) $\delta$ (ppm): 7,82 - 7,75 (m, 2H, $\left.\mathrm{H}_{\text {arom }}\right), 7,41$ - 7,29 (m, 2H, $\left.\mathrm{H}_{\text {arom }}\right)$.

${ }^{13}$ C-NMR (75 MHz, DMSO-d 6 ) $\delta$ (ppm): 154,9 (C-1), 128,8 (C-4), 127,6 (C-3), 123,8 (C2). 
ESI-MS: $\mathrm{m} / \mathrm{z}(\%)=173,0(100)[\mathrm{M}-\mathrm{Na}]^{-}, 368,9(8)[2 \mathrm{M}-\mathrm{Na}]^{-}$.

\section{3-Brombutylthioacetat (8)}

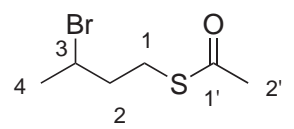

Eine Lösung aus 1,3-Dibrombutan (4,32 g, 2,40 mL, 20,0 mmol, 1,0 Äq.), Thioessigsäure (2,26 g, 2,13 mL, 29,7 mmol, 1,5 Äq.) und Kaliumcarbonat (3,99 g, 28,9 mmol, 1,45 Äq.) in THF $(60 \mathrm{~mL})$ wurde für $48 \mathrm{~h}$ bei Raumtemperatur gerührt. Die Reaktion wurde durch Zugabe von Wasser $(20 \mathrm{~mL})$ beendet, die organische Phase abgetrennt und die wässrige Phase mit EtOAc extrahiert. Nach Trocknen der vereinten organischen Phasen über $\mathrm{Na}_{2} \mathrm{SO}_{4}$ und Entfernen des Lösungsmittels unter vermindertem Druck konnte das Produkt in Form eines gelben Öls isoliert werden (1,475 g, 7,0 mmol, 35 \%).

${ }^{1}$ H-NMR $\left(300 \mathrm{MHz}, \mathrm{CDCl}_{3}\right) \delta$ (ppm): 4,09 - 4,20 (m, 1H, 3-H), 2,88 - 3,14 (m, 2H, 1-H), 2,32 (s, 3H, 2'-H), 1,98 - 2,07 (m, 2H, 2-H), 1,72 (d, $J=6,7 \mathrm{~Hz}, 3 \mathrm{H}, 4-\mathrm{H})$.

\section{3-Hydroxypropylthioacetat (9)}

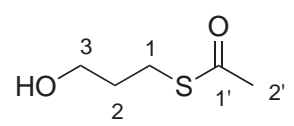

Die Synthese von 9 erfolgte nach der Vorschrift von Hagiya. ${ }^{[132]}$ Allylalkohol $(2,90 \mathrm{~g}$, $50,0 \mathrm{mmol}, 1,00 \mathrm{Äq}$.) in Chloroform $(15 \mathrm{~mL})$ wurde auf $60^{\circ} \mathrm{C}$ erhitzt. Thioessigsäure (4,00 g, 52,5 mmol, 1,05 Äq.) wurde tropfenweise zugegeben. Nach Abschluss der Zugabe wurde die Reaktionsmischung bei $50-60^{\circ} \mathrm{C}$ für $2 \mathrm{~h}$ gerührt. Das Lösungsmittel sowie der Thioessigsäureüberschuss wurden im Vakuum entfernt und das Produkt als farblose Flüssigkeit (6,65 g, 49,6 mmol, $99 \%$ ) erhalten.

${ }^{1} \mathbf{H}-\mathbf{N M R}\left(300 \mathrm{MHz}, \mathrm{CDCl}_{3}\right) \delta(\mathrm{ppm}): 3,64\left(\mathrm{t}, J=5,9 \mathrm{~Hz}, 2 \mathrm{H}, \mathrm{HO}-\mathrm{CH}_{2}\right), 3,00(\mathrm{t}, J=6,8 \mathrm{~Hz}$, $2 \mathrm{H}, \mathrm{AcS}-\mathrm{CH}_{2}$ ), 2,35 (s, 3H, $\left.\mathrm{CH}_{3}\right), 1,81$ (quin, $J=5,4 \mathrm{~Hz}, 2 \mathrm{H}, \mathrm{CH}_{2}$ ).

${ }^{1}$ H-NMR $\left(300 \mathrm{MHz}, \mathrm{THF}-\mathrm{d}_{8}\right) \delta$ (ppm): 3,50 (t, $\left.J=6,0 \mathrm{~Hz}, 2 \mathrm{H}, \mathrm{HO}-\mathrm{CH}_{2}\right), 2,91(\mathrm{t}$, $\left.J=7,2 \mathrm{~Hz}, 2 \mathrm{H}, \mathrm{AcS}-\mathrm{CH}_{2}\right), 2,26\left(\mathrm{~s}, 3 \mathrm{H}, \mathrm{CH}_{3}\right), 1,69$ (quin, $J=7,2 \mathrm{~Hz}, 2 \mathrm{H}, \mathrm{CH}_{2}$ ). 
${ }^{13}$ C-NMR (75 MHz, THF-d 8 ) $\delta$ (ppm): 195,6 (C-1'), 61,5 (C-3), 34,4 (C-2'), 30,9 (C-1), 27,0 (C-2).

ESI-MS: $\mathrm{m} / \mathrm{z}(\%)=157,0(100)[\mathrm{M}+\mathrm{Na}]^{+}, 117,0(24)[\mathrm{M}-\mathrm{OH}]^{+}, 135,1(8)[\mathrm{M}+\mathrm{H}]^{+}$.

\section{3-Chlorpropylthioacetat (10)}

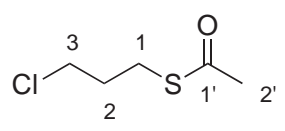

1-Brom-3-chlorpropan (5,00 g, 31,8 mmol, 1,0 Äq.) und Kaliumcarbonat (5,27 g, $38,1 \mathrm{mmol}, 1,2$ Äq.) wurden in Aceton $(60 \mathrm{~mL})$ vorgelegt. Unter Rühren wurde Thioessigsäure (2,42 g, 31,8 mmol, 1,0 Äq.) langsam zugetropft und das Reaktionsgemisch für $48 \mathrm{~h}$ bei Raumtemperatur gerührt. Anschließend wurde die Lösung filtriert und das Lösungsmittel unter vermindertem Druck entfernt. Der Rückstand wurde in Dichlormethan $(40 \mathrm{~mL})$ gelöst und mit demineralisiertem Wasser $(3 \times 20 \mathrm{~mL})$ gewaschen. Das Lösungsmittel wurde unter vermindertem Druck entfernt und die Zielverbindung in Form einer orangen Flüssigkeit (3,26 g, 21,4 mmol, 67\%) erhalten.

${ }^{1} \mathbf{H}-\mathrm{NMR}\left(300 \mathrm{MHz}, \mathrm{CDCl}_{3}\right) \delta$ (ppm): 3,57 ( $\left.t, J=6,6 \mathrm{~Hz}, 2 \mathrm{H}, 3-\mathrm{H}\right), 3,00(t, J=6,6 \mathrm{~Hz}, 2 \mathrm{H}$, $1-\mathrm{H}), 2,32$ (s, 3H, 2'-H), 2,03 (quin, $J=6,6 \mathrm{~Hz}, 2 \mathrm{H}, 2-\mathrm{H}$ ).

${ }^{13}$ C-NMR (75 MHz, CDCl 3 ) $\delta$ (ppm): 195,4 (C-1'), 43,3 (C-3), 32,2 (C-2), 30,6 (C-2"), 26,3 (C-1).

ESI-MS: $\mathrm{m} / \mathrm{z}(\%)=174,9958[\mathrm{M}+\mathrm{Na}]^{+}$.

\section{2-Methylprop-2-en-1-yl-thioacetat (11)}

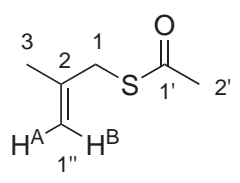

1-Chlor-2-methylprop-2-en (3,94 g, 39,1 mmol, 1,0 Äq.) und Kaliumcarbonat (7,02 g, $50,8 \mathrm{mmol}, 1,3$ Äq.) wurden in Aceton $(50 \mathrm{~mL})$ vorgelegt. Unter Rühren wurde Thioes- 
sigsäure (3,57 g, 46,9 mmol, 1,2 Äq.) langsam zugetropft und das Reaktionsgemisch für $24 \mathrm{~h}$ bei Raumtemperatur gerührt. Anschließend wurde die Lösung filtriert und das Lösungsmittel unter vermindertem Druck entfernt. Der Rückstand wurde in Dichlormethan $(40 \mathrm{~mL})$ gelöst, mit demineralisiertem Wasser $(3 \times 20 \mathrm{~mL})$ gewaschen und das Lösungsmittel unter vermindertem Druck entfernt. Die Zielverbindung wurde in Form einer dunkelbraunen Flüssigkeit (2,05 g, 15,8 mmol, 40 \%) erhalten.

${ }^{1}$ H-NMR $\left(300 \mathrm{MHz}, \mathrm{CDCl}_{3}\right) \delta(\mathrm{ppm}): 4,94\left(\mathrm{~s}, 1 \mathrm{H}, 1^{\prime \prime}-\mathrm{H}^{\mathrm{B}}\right), 4,82\left(\mathrm{~s}, 1 \mathrm{H}, 1^{\prime \prime}-\mathrm{H}^{\mathrm{A}}\right), 3,54(\mathrm{~s}$, $2 \mathrm{H}, 1-\mathrm{H}), 2,34\left(\mathrm{~s}, 3 \mathrm{H}, 2^{\prime}-\mathrm{H}\right), 1,75(\mathrm{~s}, 3 \mathrm{H}, 3-\mathrm{H})$.

${ }^{13}$ C-NMR (75 MHz, CDCl 3 ) $\delta$ (ppm): 195,2 (C-1'), 140,7 (C-2), 114,0 (C-1"), 36,0 (C-1), $30,4\left(\mathrm{C}-2^{\prime}\right), 21,1(\mathrm{C}-3)$.

ESI-MS: $\mathrm{m} / \mathrm{z}(\%)=153,0343[\mathrm{M}+\mathrm{Na}]^{+}$.

\section{Bis(1-methyl-3-thioacetylpropyl)trithiocarbonat (16)}<smiles>CC(=O)SCCC(C)SC(=S)SC(C)CCSC(C)=O</smiles>

Die Synthese erfolgte in Anlehnung an die von Aoyagi et al. entwickelte Trithiocarbonatsynthese. ${ }^{[93,94]}$ Eine Lösung von Schwefelkohlenstoff $(0,20 \mathrm{~mL}, 3,3 \mathrm{mmol}$, 1,0 Äq.) und Cäsiumcarbonat (1,08 g, 3,3 mmol, 1,0 Äq.) in DMAc (4 mL) wurde $15 \mathrm{~min}$ bei Raumtemperatur gerührt. Anschließend wurde eine Lösung von 3Brombutylthioacetat $(0,70 \mathrm{~g}, 3,31 \mathrm{mmol}, 1,0$ Äq.) in DMAc $(1 \mathrm{~mL})$ zugetropft und $24 \mathrm{~h}$ bei Raumtemperatur gerührt. Anschließend wurde das Reaktionsgemisch auf Eiswasser gegossen und die wässrige Phase mit EtOAc $(3 \times 5 \mathrm{~mL})$ extrahiert, bis die organische Phase farblos blieb. Die vereinigten organischen Phasen wurden über $\mathrm{Na}_{2} \mathrm{SO}_{4}$ getrocknet und das Lösungsmittel unter vermindertem Druck entfernt. Das Rohprodukt wurde mittels Säulenchromatographie an Kieselgel gereinigt (Pentan/EtOAc 4:1). Das RAFT-Agens konnte als schwach gelbes Öl isoliert werden (103 mg, 0,28 mmol, 17\%).

${ }^{1} \mathbf{H}-\mathbf{N M R}\left(300 \mathrm{MHz}, \mathrm{CDCl}_{3}\right) \delta$ (ppm): 4,28 - 4,17 (m, 1H, 1-H), 3,02 - 2,87 (m, 2H, 3-H), 2,32 (s, 3H, $\left.\mathrm{CH}_{3}\right), 2,05-1,80(\mathrm{~m}, 2 \mathrm{H}, 2-\mathrm{H}), 1,41\left(\mathrm{~d}, J=6,9 \mathrm{~Hz}, 3 \mathrm{H}, 1^{\prime}-\mathrm{H}\right)$.

${ }^{13}$ C-NMR (75 MHz, CDCl 3 ) $\delta$ (ppm): 222,2 (C=S), 195,3 (C=O), 45,5 (C-1), 35,6 (C-2), $30,5\left(\mathrm{CH}_{3}-\mathrm{Ac}\right), 26,5$ (C-3), 19,9 (C-1'). 
EI-MS: $\mathrm{m} / \mathrm{z}(\%)=392,9(100)[\mathrm{M}-\mathrm{Na}]^{+}$.

\section{Methyl-2-[methyl(pyridin-4-yl)carbamothioylthio]propanoat (30)}<smiles>COC(=O)C(C)SC(=S)N(C)c1ccncc1</smiles>

Die Synthese von 30 erfolgte in Anlehnung an Benaglia et al. ${ }^{[49]}$ unter Austausch der Base. 4-(Methyl-amino)-pyridin (2,16 g, $20 \mathrm{mmol}, 1,0$ Äq.) und Natriumhydrid (60\% in Mineralöl, 1,04 g, $26 \mathrm{mmol}, 1,3$ Äq.) wurden in THF $(50 \mathrm{~mL})$ gelöst und $2 \mathrm{~h}$ bei $40^{\circ} \mathrm{C}$ gerührt. Unter Eiskühlung wurde Schwefelkohlenstoff $(3,05 \mathrm{~g}, 2,43 \mathrm{~mL}, 40 \mathrm{mmol}$, 2,0 Äq.) zugetropft und die Reaktionsmischung über Nacht bei Raumtemperatur gerührt. Methyl-2-brompropionat $(5,01 \mathrm{~g}, 3,35 \mathrm{~mL}, 30 \mathrm{mmol}, 1,5 \mathrm{Äq}$.) wurde zugetropft und die Reaktionsmischung weitere $2 \mathrm{~h}$ bei Raumtemperatur gerührt. Anschließend wurde mit $\mathrm{Et}_{2} \mathrm{O}(3 \times 80 \mathrm{~mL})$ extrahiert und die vereinigten organischen Phasen mit gesättigter wässriger $\mathrm{NaHCO}_{3}$-Lösung $(2 \times 80 \mathrm{~mL})$ und gesättigter wässriger $\mathrm{NaCl}$ Lösung $(2 \times 80 \mathrm{~mL})$ gewaschen. Nach Trocknung über $\mathrm{Na}_{2} \mathrm{SO}_{4}$ wurde das Lösungsmittel in vacuo entfernt. Der Rückstand wurde mittel Säulenchromatographie an Kieselgel (Hexan/EtOAc 7:3 $\rightarrow$ 0:1) gereinigt. Das Produkt wurde als gelber Feststoff (3,55 g, $13,2 \mathrm{mmol}, 66 \%$ ) erhalten.

${ }^{1}$ H-NMR (300 MHz, $\left.\mathrm{CDCl}_{3}\right) \delta$ (ppm): 8,74 (dd, $\left.J=4,5 \mathrm{~Hz}, 1,5 \mathrm{~Hz}, 2 \mathrm{H}, \mathrm{o}-\mathrm{ArH}\right), 7,25$ (dd, $J=4,5 \mathrm{~Hz}, 1,8 \mathrm{~Hz} 2 \mathrm{H}, m-\mathrm{ArH}), 4,65\left(\mathrm{q}, J=7,4 \mathrm{~Hz}, 1 \mathrm{H}, \mathrm{CHCH}_{3}\right), 3,72\left(2 \mathrm{x} \mathrm{s}, 6 \mathrm{H}, \mathrm{NCH}_{3}\right.$, $\left.\mathrm{COOCH}_{3}\right), 1,52\left(\mathrm{~d}, J=7,5 \mathrm{~Hz}, 3 \mathrm{H}, \mathrm{CHCH}_{3}\right)$.

${ }^{13}$ C-NMR (75 MHz, $\mathrm{CDCl}_{3}$ ) $\delta$ (ppm): 196,7 (C=S), 172,3 (C=O), 151,7 (2 x C-Aryl), 121,7 (C-Aryl), 52,7 (O- $\left.\mathrm{CH}_{3}\right), 49,7\left(\mathrm{CH}_{3}-\mathrm{CH}\right), 45,3\left(\mathrm{CH}_{3}-\mathrm{N}\right), 16,9\left(\mathrm{CH}_{3}-\mathrm{CH}\right)$.

ESI-MS: $\mathrm{m} / \mathrm{z}(\%)=563,1(100)[2 \mathrm{M}+\mathrm{Na}]^{+}, 271,0(43)[\mathrm{M}+\mathrm{H}]^{+}, 293,0(32)[\mathrm{M}+\mathrm{Na}]^{+}$. 


\section{4-Vinylbenzylthioacetat (ViBET) (25)}

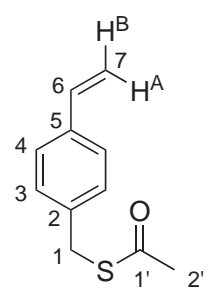

Synthese I: ${ }^{[96]} 4$-Vinylbenzylchlorid (6,63 g, 43,5 mmol, 1,0 Äq.), Thioessigsäure (3,97 g, 3,75 mL, 52,2 mmol, 1,2 Äq.) und Kaliumcarbonat (6,01 g, 52,2 mmol, 1,2 Äq.) wurden in THF $(80 \mathrm{~mL})$ für $19 \mathrm{~h}$ bei Raumtemperatur gerührt. Anschließend wurde die Reaktionsmischung im Vakuum eingeengt, der Rückstand in demineralisiertem Wasser $(100 \mathrm{~mL})$ gelöst und mit EtOAc $(3 \times 70 \mathrm{~mL})$ extrahiert. Die vereinigten organischen Phasen wurden mit gesättigter $\mathrm{NaCl}$-Lösung gewaschen, über $\mathrm{MgSO}_{4}$ getrocknet und das Lösungsmittel im Vakuum entfernt. Das Produkt wurde in Form einer orangen Flüssigkeit $(8,20 \mathrm{~g}, 42,6 \mathrm{mmol}, 98 \%)$ erhalten.

Synthese II: Thioessigsäure (1,71 g, 1,62 mL, 22,5 mmol, 1,5 Äq.) und Kaliumcarbonat $(2,49 \mathrm{~g}, 22,5 \mathrm{mmol}, 1,5$ Äq.) wurde in Aceton $(60 \mathrm{~mL})$ vorgelegt und $30 \mathrm{~min}$ bei Raumtemperatur gerührt. 4-Vinylbenzylchlorid (2,83 g, 2,11 mL, 15,0 mmol, 1,0 Äq.) wurde langsam zugetropft und die Reaktionsmischung für $30 \mathrm{~min}$ bei $40^{\circ} \mathrm{C}$ gerührt. Das Lösungsmittel wurde im Vakuum entfernt, der Rückstand in demineralisiertem Wasser $(50 \mathrm{~mL})$ gelöst und mit EtOAc $(3 \times 30 \mathrm{~mL})$ extrahiert. Die vereinigten organischen Phasen wurden mit gesättigter $\mathrm{NaCl}$-Lösung gewaschen, über $\mathrm{MgSO}_{4}$ getrocknet und das Lösungsmittel im Vakuum entfernt. Das Produkt wurde in Form einer orangen Flüssigkeit (2,88 g, 15,0 mmol, quantitativ) erhalten.

${ }^{1}$ H-NMR (300 MHz, $\left.\mathrm{CDCl}_{3}\right) \delta$ (ppm): 7,34 (d, $\left.J=8,4 \mathrm{~Hz}, 2 \mathrm{H}, 4-\mathrm{H}\right), 7,25(\mathrm{~d}, J=8,4 \mathrm{~Hz}$, $2 \mathrm{H}, 3-\mathrm{H}), 6,69(\mathrm{dd}, J=17,6 \mathrm{~Hz}, 10,9 \mathrm{~Hz} 1 \mathrm{H}, 6-\mathrm{H}), 5,72\left(\mathrm{dd}, J=17,6 \mathrm{~Hz}, 1,0 \mathrm{~Hz}, 1 \mathrm{H}, 7-\mathrm{H}^{\mathrm{A}}\right)$, $5,23\left(\mathrm{dd}, J=10,9 \mathrm{~Hz}, 0,9 \mathrm{~Hz}, 1 \mathrm{H}, 7-\mathrm{H}^{\mathrm{B}}\right), 4,11(\mathrm{~s}, 2 \mathrm{H}, 1-\mathrm{H}), 2,35\left(\mathrm{~s}, 3 \mathrm{H}, 2^{\prime}-\mathrm{H}\right)$.

${ }^{13}$ C-NMR (75 MHz, CDCl 3 ) $\delta$ (ppm): 194,9 (C-1'), 137,1 (C-2), 136,6 (C-5), 136,3 (C-6), 128,9 (C-3), 126,4 (C-4), 113,9 (C-7), 33,2 (C-1), 30,3 (C-2').

ESI-MS: $\mathrm{m} / \mathrm{z}(\%)=193,1(100)[\mathrm{M}+\mathrm{H}]^{+}, 117,1(60)\left[\mathrm{M}-\mathrm{SC}(\mathrm{O}) \mathrm{CH}_{3}\right]^{+}, 215,0(50)$ $[\mathrm{M}+\mathrm{Na}]^{+}$. 


\section{4-Vinylbenzyldithioacetat (ViBEDiT) (38)}

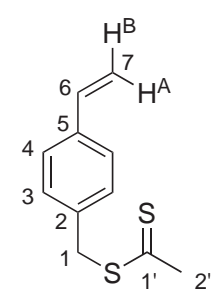

Zu Methylmagnesiumchlorid (3,0 M in THF, 10,00 mL, 30,0 mmol, 1,2 Äq.) wurde unter Eiskühlung Schwefelkohlenstoff $(2,00 \mathrm{~mL}, 33,0 \mathrm{mmol}, 1,3$ Äq.) zugetropft. Die Reaktionsmischung wurde für $26 \mathrm{~h}$ gerührt und währenddessen langsam auf Raumtemperatur erwärmt. 4-Vinylbenzylchlorid (2,30 mL, 16,5 mmol, 0,7 Äq.) wurde langsam zugetropft und die Reaktionsmischung für $2 \mathrm{~h}$ bei $40^{\circ} \mathrm{C}$ und weitere $69 \mathrm{~h}$ bei Raumtemperatur gerührt. Anschließend erfolgte die Zugabe einer weiteren Menge 4Vinylbenzylchlorid (1,2 mL, 8,5 mmol, 0,3 Äq.) tropfenweise. Die Lösung wurde für $3 \mathrm{~h}$ bei $40^{\circ} \mathrm{C}$ gerührt. Zum Beenden der Reaktion wurde die Mischung auf Eiswasser gegossen, mit Hexan $(3 \times 20 \mathrm{~mL})$ extrahiert und eingeengt. Das Rohprodukt wurde mittels Kugelrohrdestillation $\left(p=5 \cdot 10^{-4} \mathrm{mbar}, \mathrm{T}=100^{\circ} \mathrm{C}\right)$ aufgereinigt und in Form eines gelben, hochviskosen Öls $(2,18 \mathrm{~g}, 10,5 \mathrm{mmol}, 42 \%)$ erhalten.

${ }^{1}$ H-NMR (300 MHz, $\left.\mathrm{CDCl}_{3}\right) \delta$ (ppm): 7,38 (d, $\left.J=8,3 \mathrm{~Hz}, 2 \mathrm{H}, 4-\mathrm{H}\right), 7,30$ (d, $J=8,3 \mathrm{~Hz}$, $2 \mathrm{H}, 3-\mathrm{H}), 6,72(\mathrm{dd}, J=17,6 \mathrm{~Hz}, 10,9 \mathrm{~Hz}, 1 \mathrm{H}, 6-\mathrm{H}), 5,76(\mathrm{dd}, J=17,6 \mathrm{~Hz}, 0,9 \mathrm{~Hz}, 1 \mathrm{H}$, $\left.7-\mathrm{H}^{\mathrm{A}}\right), 5,27\left(\mathrm{dd}, J=10,9 \mathrm{~Hz}, 0,9 \mathrm{~Hz}, 1 \mathrm{H}, 7-\mathrm{H}^{\mathrm{B}}\right), 4,47$ (s, 2H, 1-H), 2,87 (s, 3H, 2'-H).

${ }^{13}$ C-NMR $\left(75 \mathrm{MHz}, \mathrm{CDCl}_{3}\right) \delta$ (ppm): 232,1 (C-1'), 137,0 (C-2), 136,2 (C-5), 134,6 (C-6), 129,2 (C-3), 126,4 (C-4), 114,1 (C-7), 41,7 (C-1), 38,8 (C-2').

EI-MS: $\mathrm{m} / \mathrm{z}(\%)=117,1(100)\left[\mathrm{M}-\mathrm{SC}(\mathrm{S}) \mathrm{CH}_{3}\right]^{+}, 208,0(10)[\mathrm{M}+\mathrm{H}]^{+}$.

\section{4-Ethylbenzylthioacetat (EBET) (39)}

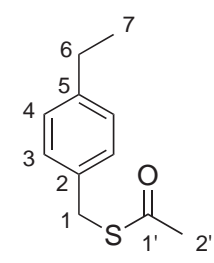


Zu Thioessigsäure $(0,46 \mathrm{~g}, 6,0 \mathrm{mmol}, 1,2 \mathrm{Äq}$.) in THF (2 mL) wurde Triethylamin $(0,63 \mathrm{~g}$, 6,2 mmol, 1,3 Äq.) langsam zugetropft. Anschließend wurde 4-Ethylbenzylchlorid $(0,76 \mathrm{~g}, 4,9 \mathrm{mmol}, 1,0$ Äq.) zugetropft und die Reaktionsmischung für $18 \mathrm{~h}$ bei Raumtemperatur gerührt. Das Lösungsmittel wurde im Vakuum entfernt, der Rückstand in demineralisiertem Wasser $(2 \mathrm{~mL})$ gelöst und mit Dichlormethan $(3 \times 10 \mathrm{~mL})$ extrahiert. Das Lösungsmittel wurde im Vakuum entfernt und das Produkt in Form einer braunen Flüssigkeit (0,96 g, 4,9 mmol, quantitativ) erhalten.

${ }^{1} \mathrm{H}$ NMR (300 MHz, $\left.\mathrm{CDCl}_{3}\right) \delta(\mathrm{ppm}): 7,22(\mathrm{~d}, J=8,2 \mathrm{~Hz}, 2 \mathrm{H}, 4-\mathrm{H}), 7,14(\mathrm{~d}, J=8,2 \mathrm{~Hz}, 2 \mathrm{H}$, 3-H), 4,11 (s, 2H, 1-H), 2,63 (q, $J=7,6 \mathrm{~Hz}, 2 \mathrm{H}, 6-\mathrm{H}), 2,35\left(\mathrm{~s}, 3 \mathrm{H}, 2^{\prime}-\mathrm{H}\right), 1,23(\mathrm{t}, J=7,6 \mathrm{~Hz}$, $3 \mathrm{H}, 7-\mathrm{H})$.

${ }^{13}$ C NMR (75 MHz, CDCl 3 ) $\delta$ (ppm): 195,1 (C-1'), 143,3 (C-2), 134,7 (C-5), 128,7 (C-4), 128,1 (C-3), 33,2 (C-1), 30,3 (C-6), 28,4 (C-2'), 15,5 (C-7).

EI-MS: $\mathrm{m} / \mathrm{z}(\%)=119,1(100)$ [M-SAc] $^{+}, 194,1(24)[\mathrm{M}]^{+}, 151,1(19)[\mathrm{M}-\mathrm{Ac}]^{+}$.

\section{4-Vinylbenzylbenzolthiosulfonat (ViBSuT) 26}

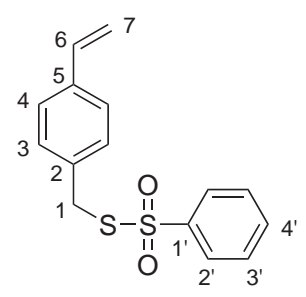

Die Synthese wurde zur Reaktionsoptimierung mehrfach wiederholt und dabei geringfügig verändert. Die genauen Zusammensetzungen sind Tabelle 5.1 und Tabelle $5.2 \mathrm{zu}$ entnehmen. Hier wird exemplarisch das Vorgehen bei der Synthese A11 beschrieben.

Eine Mischung aus frisch destilliertem 4-Vinylbenzylchlorid (9,71 g, 64,0 mmol, 1,0 Äq.), Natriumbenzolthiosulfonat (25,14 g, $128,0 \mathrm{mmol}, 2,0$ Äq.), Aliquat $336^{\circledR}$ $(1,31 \mathrm{~g}, 3,2 \mathrm{mmol}, 0,05 \mathrm{~mol}-\%)$ und Wasser $(320 \mathrm{~mL})$ wurde bei $65^{\circ} \mathrm{C}$ für $380 \mathrm{~min}$ gerührt. Anschließend wurde die Reaktionsmischung auf Raumtemperatur abgekühlt und mit EtOAc $(3 \times 100 \mathrm{~mL})$ extrahiert. Die vereinigten organischen Phasen wurden mit gesättigter $\mathrm{NaCl}$-Lösung gewaschen, über $\mathrm{MgSO}_{4}$ getrocknet und im Vakuum eingeengt. Das Rohprodukt wurde mittels Säulenchromatographie an Kieselgel gereinigt (Hexan/EtOAc 20:1 $\rightarrow$ 0:1) und das Monomer konnte als weißer Feststoff in 95\%-iger 
Reinheit isoliert werden (3,11 g, 10,7 mmol, $17 \%)$.

${ }^{1} \mathbf{H}$ NMR $\left(300 \mathrm{MHz}, \mathrm{CDCl}_{3}\right) \delta$ (ppm): 7,82 (dd, $\left.J=8,7 \mathrm{~Hz}, 1,2 \mathrm{~Hz}, 2 \mathrm{H}, 2^{\prime}-\mathrm{H}\right), 7,58$ (tt, $\left.J=7,5 \mathrm{~Hz}, 1,2 \mathrm{~Hz}, 1 \mathrm{H}, 4^{\prime}-\mathrm{H}\right), 7,46\left(\mathrm{tt}, J=7,2 \mathrm{~Hz}, 1,2 \mathrm{~Hz}, 2 \mathrm{H}, 3^{\prime}-\mathrm{H}\right), 7,25$ (d, $J=8,1 \mathrm{~Hz}$, 2H, 4-H), 7,13 (d, $J=8,4 \mathrm{~Hz}, 2 \mathrm{H}, 3-\mathrm{H}), 6,65(\mathrm{dd}, J=17,7 \mathrm{~Hz}, 11,1 \mathrm{~Hz}, 1 \mathrm{H}, 6-\mathrm{H}), 5,71(\mathrm{dd}$, $J=17,4 \mathrm{~Hz}, 0,9 \mathrm{~Hz}, 1 \mathrm{H}, 7-\mathrm{H}), 5,25(\mathrm{dd}, J=10,8 \mathrm{~Hz}, 0,9 \mathrm{~Hz}, 1 \mathrm{H}, 7-\mathrm{H}), 4,26$ (s, 2H, 1-H).

${ }^{13}$ C NMR (75 MHz, $\left.\mathrm{CDCl}_{3}\right) \delta$ (ppm): 144,9 (C-1'), 137,4 (C-2), 136,5 (C-5), 136,0 (C-6), 133,4 (C-4'), 129,3 (C-3'), 129,1 (C-3), 126,8 (C-2'), 126,5 (C-4), 114,5 (C-7), 40,2 (C-1).

ESI-MS: $\mathrm{m} / \mathrm{z}(\%)=313,0(100)[\mathrm{M}+\mathrm{Na}]^{+}, 308,1(50)\left[\mathrm{M}+\mathrm{NH}_{4}\right]^{+}, 291,0(2)[\mathrm{M}+\mathrm{H}]^{+}$.

\subsection{Nummerierung}

Die unterschiedlichen Optimierungsversuche für die ViBSuT-Synthese sind mit einem "S“ für Synthese und einer fortlaufenden zweistelligen Nummer gekennzeichnet. Die RAFT-Polymerisationen tragen ein „, $R^{\prime \prime}$, ebenfalls gefolgt von einer zweistelligen fortlaufenden Nummer. Wurden mehrere Proben mit der gleichen Einwaage, aber unterschiedlichen Polymerisationszeiten hergestellt, erhalten diese zusätzlich einen Buchstaben (z. B. R03A-F). Polymerisationen, die mit einem Comonomer oder einer comonomerähnlichen Substanz durchgeführt wurden, sind mit dem Buchstaben „, $\mathrm{M}$ “ für Monomer gekennzeichnet. Proben, die in der Elementaranalyse untersucht wurden, haben darüber hinaus eine griechische Nummer (z. B. M18B-II).

\subsection{Polymerisationen}

Sämtliche Monomere, die bei SATP-Bedingungen $\left(25^{\circ} \mathrm{C}, 1,013\right.$ bar) flüssig sind, wurden mittels 3 Einfrieren-Evakuieren-Auftauen-Zyklen (engl. freeze-pump-thaw) entgast. Bei Polymerisationen in Lösung wurde das Lösungsmittel ebenfalls entgast. Comonomermengen über $200 \mathrm{mg}$ wurden entgast oder direkt mit den übrigen flüssigen Komponenten eingewogen und entgast. Diese entgasten FLüssigkeiten wurde in einer mit Argon befüllten Glove-Box (Sauerstoffgehalt unter 4 ppm) mit dem Initiator und ggf. RAFT-Agens versetzt und in die Reaktionsgefäße aufgeteilt. Diese wurden außerhalb der Box in einem temperierten Heizblock (AIBN $(60,0 \pm 0,1)^{\circ} \mathrm{C}$, ACCN 
$\left.(80,0 \pm 0,1)^{\circ} \mathrm{C}\right)$ erwärmt und nach Ablauf der gewünschten Reaktionszeit in einem Eisbad abgeschreckt und entleert. Der Umsatz wurde gravimetrisch bestimmt.

\subsection{Aufbau des Hochdruck-Laborautoklaven ${ }^{1}$}

Die Polymerisation von Butadien wird in einem Hochdruck-Laborautoklav „Modell II“ der Firma "Carl Roth“ durchgeführt. Dieser Versuchsautoklav besteht aus äußerst korossionsbeständigem V4A-Edelstahl (Werkstoffnummer 1.4571) und ist für alle Reaktionen, bei denen ein rascher Druckanstieg zu erwarten ist, geeignet.

Der Versuchsautoklav ist aus drei Komponenten aufgebaut: Dem zylindrischen Autoklavenbecher mit flachem Boden (innen wie außen), dem Autoklavenkopf und dem Zwischenstück mit Tauchrohr. Der gesamte Reaktionsaufbau ist bis maximal 100 bar ausgelegt. Flachdichtungen aus Polytetrafluorethylen (PTFE) gewährleisten eine hohe Dichtigkeit bis $180^{\circ} \mathrm{C}$ Arbeitstemperatur. Verschraubungen, die mit diesen PTFEDichtungen ausgestattet sind, werden mit einem Schraubenschlüssel handfest angezogen, während Verschraubungen mit Feinsilberdichtungen fest angezogen werden. Gewinde werden vor dem Zusammenschrauben mit Teflon-Spray eingesprüht, um ein Kaltverschweißen der Edelstahlbauteile zu vermeiden.

Der Autoklavenkopf besitzt drei seitliche G 1/4" Gewindeöffnungen, die für die Aufnahme von Zubehör geeignet sind. An einer dieser Gewindeöffnungen ist ein Gewindeadapter montiert, über den ein Zwei-Wege-Nadelventil der Firma Swagelok angeschlossen ist, welches das Ablassen von Gasen zur Verbrennung ermöglicht. An der zweiten Gewindeöffnung ist der Temperaturfühler montiert, welcher direkt die Temperatur der Reaktionsmischung ermittelt und das Heizelement ansteuert. Zur Abdichtung wird eine Feinsilberdichtung verwendet. Der Fühler enthält ein $\mathrm{Fe} / \mathrm{Cu} / \mathrm{Ni}$ Thermoelement, welches vorsichtig um $90^{\circ}$ gebogen wurde, da es bei mechanischer Überbeanspruchung brechen kann. Die dritte Gewindeöffnung ist nicht belegt, bietet aber die Möglichkeit z.B. ein Manometer anzuschließen.

Auf dem Autoklavenkopf sitzt ein Zwischenstück, welches eine Berstscheibe 28.100 enthält. Diese Berstscheibe ist mittels einer Fensilberdichtung mit der Wölbung nach außen in der oberen Öffnung des Zwischenstücks montiert. Sie zerplatzt bei einem Druck von 100 bar $\pm 10 \%$.

Der Autoklavenkopf mit seinen Zu- und Ablässen stellt das Kernstück des Autoklaven dar. Eventuelle Undichtigkeiten an einer oder mehreren der zahlreichen Verschraubun-

\footnotetext{
${ }^{1}$ Teilweise aus früheren Arbeiten übernommen ${ }^{[23]}$
} 
gen können mit Hilfe von Ulith, einem Lecksuchspray, lokalisiert und anschließend behoben werden. Alle relevanten technischen Daten finden sich in Tabelle 11.2 und Tabelle 11.1.

Tabelle 11.1 Technische Daten des Autoklavenbechers

\begin{tabular}{cc}
\hline Leervolumen [mL] & 350 \\
Arbeitsvolumen [mL] & 300 \\
Höhe [mm] & 300 \\
Gewicht [g] & 1900 \\
Arbeitstemperatur $\left[{ }^{\circ} \mathrm{C}\right]$ & -60 bis +300 \\
Max. Arbeitsdruck [bar] & 100 \\
Prüfdruck bei $20^{\circ} \mathrm{C}$ & 300 \\
Außendurchmesser [mm] & 50 \\
Innendurchmesser [mm] & 40 \\
Außendurchmesser am Kopf [mm] & 64 \\
\hline
\end{tabular}

Autoklavenbecher, -kopf und Zwischenstück bilden den Reaktionsraum für die Polymerisation von Monomeren, die bei Standardbedingungen gasförmig sind (wie Butadien).

Tabelle 11.2 Technische Daten des Autoklavenkopfes

\begin{tabular}{cc}
\hline Gewicht $[\mathrm{g}]$ & 1250 \\
Arbeitstemperatur $\left[{ }^{\circ} \mathrm{C}\right]$ & -60 bis +300 \\
Max. Arbeitsdruck [bar] & 200 \\
Außendurchmesser [mm] & 64 \\
\hline
\end{tabular}

\subsubsection{Zubehör des experimentellen Aufbaus}

Der Hochdruckautoklav wurde durch diverses Zubehör ergänzt, um eine komfortable und funktionsgerechte Bedienung zu gewährleisten. Der komplette Versuchsaufbau ist in Abbildung 11.1 dargestellt. Sämtliche Komponenten werden untereinander durch einen flexiblen, gasdichten PFA-Schlauch, der bei $20^{\circ} \mathrm{C}$ eine Druckbeständigkeit von maximal 100 bar besitzt, oder einen Gewebeschlauch, der bis 10 bar druckbeständig ist 
und für Stickstoff benutzt wird, verbunden. Die Reaktionstemperatur wird durch den externen Temperaturregler WRX 2000 reguliert, der die Heizleistung an der Heizhaube S 30 einstellt. Diese Heizhaube umschließt den Autoklavenbecher komplett und sorgt für eine gleichmäßige Erwärmung. Die Integration eines Rührwerks bietet die Möglichkeit durch magnetische Kraftübertragung einen Tablettenrührmagneten anzutreiben, der für eine gute Durchmischung der Reaktionslösung sorgt.

Die Stickstoff-Gasflasche ist über ein Druckminderungsventil an den apparativen Aufbau angeschlossen. Für die Butadien-Gasflasche wurde ein Flaschendruckminderer für Sondergase der Firma „Messer Griesheim“ verwendet. Er besteht aus Edelstahl und zeichnet sich durch eine hohe Widerstandsfähigkeit gegenüber korrosiven Gasen aus und ist somit optimal für Butadien geeignet. Der direkte Anschluss an die ButadienGasflasche und die integrierte Spüleinrichtung erleichtern die Handhabung. Der technische Stickstoff wird mit einem Druck von maximal 4 bar vom Flaschendruckminderer der Stickstoff-Gasflasche über einen Drei-Wege-Hahn (H1) geleitet und steht damit sowohl dem Reaktionsautoklaven als auch dem Flaschendruckminderer der ButadienGasflasche zur Verfügung, welcher über das Ventil der Stickstoffzufuhr (B6) mit Stickstoff gespült werden kann. Ein Ablass- (B4), ein Überdruck- (B5) und ein Druckminderungsventil (B2) vervollständigen den Flaschendruckminderer. Das Überdruck-, das Ablass- sowie das Stickstoffzufuhrventil sind mit Schlauchadaptern zum Anschluss von Gewebeschläuchen mit einem Innendurchmesser von $8 \mathrm{~mm}$ ausgerüstet. Die Gewebeschläuche sind mit Schlauchschellen gesichert. Der PFA-Schlauch ist dem Druckminderer über einen Swagelok-Verschluss mit $6 \mathrm{~mm}$ Innendurchmesser angeschlossen. Der Füllautoklave zur gezielten Massenbestimmung des Monomers kann über ein Kupplungssystem mit dem Flaschendruckminderer der Butadien-Gasflasche verbunden werden und so mit Stickstoff gespült oder mit Butadien befüllt werden. Der Füllautoklav ist aus Edelstahl hergestellt (Werkstoffnummer 1.4542) und besitzt eine Druckbeständigkeit bis 500 bar. Die technischen Daten sind in Tabelle 11.3 aufgelistet.

Tabelle 11.3 Technische Daten des Füllautoklaven

\begin{tabular}{cc}
\hline Gewicht [g] & 830 \\
Max. Arbeitsdruck [bar] & 500 \\
Außendurchmesser [mm] & 22 \\
Höhe [mm] & 185 \\
\hline
\end{tabular}




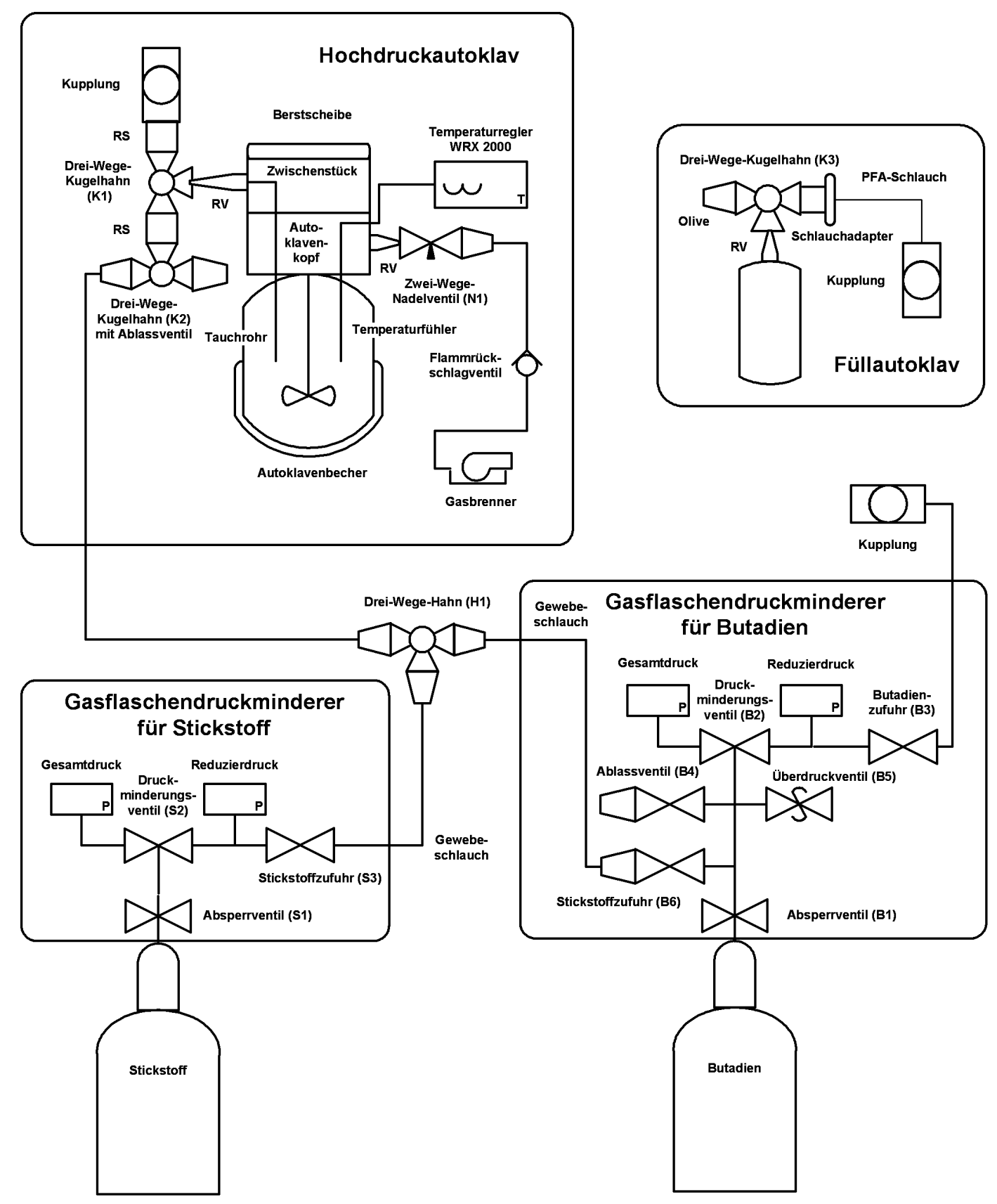

Abbildung 11.1 Schematische Darstellung des kompletten apparativen Aufbaus. $\mathrm{RS}=$ Rohrstück, $\mathrm{RV}=$ Rohrverschraubung . 
In der Deckenkappe des Füllautoklaven ist eine Reduzierverschraubung eingefasst, über die der Drei-Wege-Kugelhahn (K1) angeschlossen ist (abgedichtet mit einem Kupferring). An diesem Kugelhahn ist über ein Rohrstück ein Kupplungsgegenstück montiert, an welches der Füllautoklav angeschlossen werden kann. Darüber hinaus wurde über ein zweites Rohrstück ein weiterer Drei-Wege-Kugelhahn (K2) angeschlossen, der über den Drei-Wege-Hahn (H1) mit der Stickstoffflasche verbunden ist und ein Ablassventil zur Probenentnahme enthält. Es ist nicht möglich, Butadien direkt aus der Gasflasche in den Reaktionsautoklaven zu leiten, sondern es ist immer der Weg über den Füllautoklaven notwendig. Dies hat den Vorteil, dass die Wägung des abgekuppelten Füllautoklaven im Milligrammbereich mit einer gängigen Laborwaage möglich ist um die Masse des einkondensierten Butadiens zur Umsatzberechnung zu bestimmen. Die Anschaffung einer Waage, die den kompletten Reaktionsaufbau auf Milligramm genau wiegen kann, entfällt. Außerdem ist eine bessere Reproduzierbarkeit der Experimente gewährleistet, da die Butadienmenge unter Verwendung des Füllautoklaven nur um wenige Gramm variiert. Ist das Leergewicht des Füllautoklaven bekannt, ist es außerdem möglich, die einkondensierte Butadienmenge abzuschätzen, so dass eine bestmögliche Anpassung der Initiator- und RAFT-Agens-Konzentration erfolgen kann. Bei einem direkten Befüllen des Reaktionsautoklavs ist kein Eingreifen mehr möglich und die Varianz der verwendeten Butadienmenge ist größer. Nichtsdestotrotz wurde i.d.R. von einer Butadienmenge von $18 \mathrm{~g}$ ausgegangen und die übrigen Einwaagen darauf abgestimmt. So konnte der Reaktionsautoklav bereits während des Einkondensierens vorbereitet werden.

\subsubsection{Versuchsdurchführung}

In einem Dewargefäß aus Chromnickelstahl ( $\mathrm{V}=2 \mathrm{~L})$ wird eine Aceton-Trockeneismischung mit einer Temperatur von $-20^{\circ} \mathrm{C}$ hergestellt. Der Autoklavenbecher wird mit der benötigten Menge Toluol, AIBN und evtl. RAFT-Agens oder Comonomer befüllt. Initiator und RAFT-Agens werden auf einer Analysenwaage der Firma "Sartorius" (Analytic AC 210 S) in ein Schnappdeckelglas eingewogen. Das Toluol wird volumetrisch mit zwei Vollpipette $((50,00 \pm 0,35) \mathrm{mL}$ und $(20,0 \pm 0,2) \mathrm{mL})$ abgemessen. Ein Tablettenrührmagnet wird hinzugegeben, die Gewinde werden mit Teflonspray gefettet und der Autoklavenkopf wird mit Hilfe von 2 Schraubenschlüsseln Nr. 55 angezogen. Der Füllautoklav wird über das Kupplungssystem mit dem Gasflaschendruckminderer der Butadien-Gasflasche (B3) verbunden und über die Hähne S1-S3, H1, B6, B2, B3 und K3 mit Stickstoff gespült, um alte Butadien-Reste zu entfernen. Dazu wird temporär ein 
Gewebeschlauch auf die Olive des Füllautoklaven gesteckt, der in die Abzugsabluftanlage führt, und der Drei-Wege-Kugelhahn (K3) mehrmals betätigt.

Der Reaktionsautoklav wird über die Ventile S1-S3, H1, K2 und K1 über das Steigrohr mit Stickstoff geflutet, um Sauerstoffreste aus der Reaktionslösung und dem Reaktionsraum zu entfernen. Bei geschlossenem Ventil K1 wird das Zwei-Wege-Nadelventil N1 geöffnet und das Gas strömt über einen Gewebeschlauch in den Abzug. Durch abwechselndes Öffnen und Schließen von K1 und N1 wird der Reaktionsautoklav mehrmals mit Stickstoff gespült.

Anschließend wird die Stickstoffflasche geschlossen und die Butadien-Gasflasche geöffnet. Über die in Richtung des Füllautoklaven geöffneten Ventile B1-B3 und K3 wird Butadien in dem Füllautoklaven einkondensiert. Nach circa 20 min wird der DreiWege-Hahn K3 geschlossen und der Füllautoklav abgekuppelt. Dieser wird nun auf einer Waage (Sartorius CPA3202S) gewogen und die Gesamtmasse notiert.

Der Füllautoklav wird wieder über die Kupplung angeschlossen, der Reaktionsautoklav in dem Kühlbad auf $-20^{\circ} \mathrm{C}$ temperiert und die Ventile K3, K2 und K1 in Richtung Reaktionsautoklav geöffnet. Mit einer Heißluftpistole wird der Füllautoklav auf Raumtemperatur erwärmt und so das Butadien verdampft und über das Tauchrohr dem Reaktionsautoklaven zugeführt. Das Butadien kondensiert somit in dem Reaktionsautoklaven erneut. Nach Beendigung dieses Arbeitsschritts werden die Hähne K1, K2 und K3 geschlossen, der Füllautoklav abgekuppelt und durch Gegenwiegen des leeren Füllautoklavs die Masse des überführten Butadiens bestimmt. Bei Bedarf wird der Prozess des Einkondensierens wiederholt.

Abschließend wird die Butadien-Gasflasche geschlossen und das Reduzierventil über die Hähne S1-S3, H1 und B6 mit Stickstoff gespült, um eventueller Korrosion vorzubeugen. Sämtliche Hähne werden geschlossen und der Reaktionsautoklav in die Heizhaube S 30 gestellt. Die Rührgeschwindigkeit wird an der Heizhaube und die Temperaturvorwahl am Temperaturregler eingestellt und die Polymerisation wird gestartet. Während der Polymerisation besteht die Möglichkeit, Proben der Reaktionslösung zu entnehmen und in Schnappdeckelgläsern aufzufangen, um den Reaktionsverlauf untersuchen zu können. Dazu wird der Drei-Wege-Kugelhahn K1 in Richtung K2 geschaltet. Über das Ablassventil des Hahns K2 kann nun die Probe entnommen und direkt in ein Schnappdeckelglas gefüllt werden. Nach der Entnahme der gewünschten Probemenge werden die Ventile wieder geschlossen.

Zum Beenden der Polymerisation wird die Heizhaube ausgeschaltet und der Reaktionsautoklav in einem Eisbad auf $0-5^{\circ} \mathrm{C}$ abgekühlt. Über das Nadelventil N1 entweicht gasförmiges Rest-Monomer über einen PFA-Schlauch und ein Flammrückschlagventil 
zu einem Gasbrenner, der das Gas verbrennt. Anschließend wird der Autoklavenkopf vom Autoklavenbecher abmontiert und die Polymerlösung in einen Rundkolben gegossen. Die Reinigung des Reaktionsautoklaven erfolgt mit THF und Aceton. 



\section{Abkürzungen}

AA

ACCN

AIBN

AM

AN

ATRP

$\mathrm{BD}$

BHT

BR

$C_{\text {tr }}$

$Đ$

$d$

DBZDL

$\delta$

DMAc

DMAP

DMF
Acrylsäure

1,1'-Azobis(cyclohexancarbonitril)

2,2'-Azo-bis(isobutyronitril)

Acrylamid

Acrylonitril

atom transfer radical polymerization

1,3-Butadien

3,5-Di-tert-butyl-4-hydroxytoluol

butadiene rubber

Übertragungskonstante

Dispersität

Anzahl der bei der Terminierung gebildeten Polymerketten

Dibutylzinndilaurat

chemische Verschiebung

$N, N$-Dimethylacetamid

4-(Dimethylamino)-pyridin

$N, N$-Dimethylformamid 


\begin{tabular}{|c|c|}
\hline DMSO & Dimethylsulfoxid \\
\hline EBET & 4-Ethylbenzylthioacetat \\
\hline$\varepsilon$ & Dehnung \\
\hline ESR & electron spin resonance (Elektronenspinresonanz) \\
\hline ETA & Ethylthioacetat \\
\hline $\mathrm{Et}_{2} \mathrm{O}$ & Diethylether \\
\hline EtOAc & Ethylacetat (Essigsäureethylester) \\
\hline GPC & Gelpermeationschromatographie \\
\hline HPMAM & Hydroxypropylmethacrylamid \\
\hline$k_{\text {add }}$ & Geschwindigkeitskoeffizient der Addition \\
\hline$k_{\mathrm{d}}$ & Geschwindigkeitskoeffizient des Initiatorzerfalls \\
\hline$k_{\text {frag }}$ & Geschwindigkeitskoeffizient der Fragmentierung \\
\hline$k_{\mathrm{i}}$ & $\begin{array}{l}\text { Geschwindigkeitskoeffizient des ersten Propagationsschritts } \\
\text { (Abgangsgruppe) }\end{array}$ \\
\hline$k_{\mathrm{p}}$ & Geschwindigkeitskoeffizient der Propagation \\
\hline$k_{\mathrm{s}}$ & $\begin{array}{l}\text { Geschwindigkeitskoeffizient des ersten Propagationsschritts } \\
\text { (Initiator) }\end{array}$ \\
\hline$k_{\mathrm{t}, \mathrm{d}}$ & $\begin{array}{l}\text { Geschwindigkeitskoeffizient der Terminierung durch Dispro- } \\
\text { portionierung }\end{array}$ \\
\hline$k_{\mathrm{t}, \mathrm{k}}$ & $\begin{array}{l}\text { Geschwindigkeitskoeffizient der Terminierung durch Kombi- } \\
\text { nation }\end{array}$ \\
\hline LAM & less activated monomers, weniger aktivierte Monomere \\
\hline LM & Lösungsmittel \\
\hline MAA & Methacrylsäure \\
\hline MADIX & Macromolecular Design via the Interchange of Xanthates \\
\hline
\end{tabular}


MA

MAM

MMA

$\bar{M}_{\mathrm{n}}$

$\bar{M}_{\mathrm{n}}^{\text {theo }}$

$\bar{M}_{\mathrm{n}}^{\text {theo }}(\mathrm{T})$

$M_{\mathrm{p}}$

$\bar{M}_{\mathrm{w}}$

NBR

NIPAM

NMP

NMR

NR

NVC

NVP

PRE

PTC

RAFT

RI

RS

RT

RV

SATP
Methylacrylat

more activated monomers, stärker aktivierte Monomere

Methylmethacrylat

Zahlenmittelwert der Molmasse

Theoretischer Zahlenmittelwert der Molmasse

Theoretischer Zahlenmittelwert der Molmasse unter Berücksichtigung der Terminierung

Peakmolmasse

Massenmittelwert der Molmasse

nitrile butadiene rubber, Acrylnitril-Butadien-Kautschuk

$N$-Isopropylacrylamid

nitroxide-mediated polymerization

nuclear magnetic resonance (Kernmagnetische Resonanz)

natural rubber, Naturkautschuk

$N$-Vinylcarbazol

$N$-Vinylpyrrolidon

persistent radical effect

'phase transfer catalyst', Phasentransferkatalysator

Reversibler Additions-Fragmentierungs-Kettentransfer

'refractive index', Brechungsindex

Rohrstück

Raumtemperatur (ca. $22^{\circ} \mathrm{C}$ )

Rohrverbindung

standard ambient temperature and pressure 
SBR

$\sigma$

Sty

THF

TMS

V70

VAc

ViBA

ViBEDiT

ViBET styrene butadiene rubber, Styrol-Butadien-Kautschuk

Zugspannung

Styrol

Tetrahydrofuran

Trimethylsilyl

2,2'-Azobis-(4-methoxy-2,4-dimethylvaleronitril)

Vinylacetat

4-Vinylbenzylacetat

4-Vinylbenzyldithioacetat

4-Vinylbenzylthioacetat 


\section{Literaturverzeichnis}

[1] Gesamtverband Kunststoffverarbeitende Industrie e.V., Kunststoffverarbeitung 2012.

[2] Wirtschaftsvereinigung Kunststoff, WVK Konjunkturbericht Deutsche Kunststoffindustrie 2011.

[3] International Rubber Study Group, Statistical Summary of World Rubber Situation, Techn. Ber., 2013.

[4] H. Domininghaus, Kunststoffe, (Hrsg.: P. Elsner, P. Eyerer, T. Hirth), Springer, Heidelberg, 2012, 1203-1268.

[5] International Rubber Study Group, General Q \& A, 2013.

[6] L. H. Baekeland, Method of Making Insoluble Products of Phenol and Formaldehyde, 1907.

[7] Bakelite $\mathrm{GmbH}$, Firmengeschichte.

[8] G. Abts, Einführung in die Kautschuktechnologie, Carl Hanser Verlag, München, 2007, 45-51.

[9] B. Tieke, Makromolekulare Chemie, Wiley-VCH, Weinheim, 2005, 1-368.

[10] H. Kloppenburg, T. Groß, M. Mezger, C. Wrana, Chem. Unserer Zeit 2009, 43, 392 406.

[11] K. Matyjaszewski, W. A. Baunecker, in Macromolecular Engineering: Volume 1, 
(Herausgegeben von K. Matyjaszewski, Y. Gnanou, L. Leibler), WILEY-VCH Verlag GmbH \& Co. KGaA, Weinheim, 2007, 161-215.

[12] G. Moad, Y. Chong, A. Postma, E. Rizzardo, S. H. Thang, Polymer 2005, 46(19), 8458-8468.

[13] G. F. Meijs, E. Rizzardo, Makromol. Chem., Rapid Commun. 1988, 9, 547- 551.

[14] G. F. Meijs, E. Rizzardo, S. H. Thang, Macromolecules 1988, 21(10), 3122-3124.

[15] G. Moad, E. Rizzardo, S. H. Thang, Acc. Chem. Res. 2008, 41(9), 1133-1142.

[16] L. H. Peebles, Molecular Weight Distributions in Polymers, (Hrsg.: H. F. Mark, E. H. Immergut), John Wiley \& Sons, Inc., New York, 1971, 27-33.

[17] T. Hofe, Molmassenbestimmung über die universelle Kalibration, Techn. Ber., PSS, Mainz, 2005.

[18] T. Hofe, Warum ist die GPC eine Relativmethode?, Techn. Ber., PSS, Mainz, 2004.

[19] S. F. Sun, Physical Chemistry of Macromolecules, John Wiley \& Sons, Inc., New York, 1994, 425-429.

[20] W. Radke, in Macromolecular Engineering: Volume 3, (Herausgegeben von K. Matyjaszewski, Y. Gnanou, L. Leibler), WILEY-VCH Verlag GmbH \& Co. KGaA, Weinheim, 2007, 1881-1936.

[21] R. Houwink, J. Prakt. Chem. 1940, 157, 15-18.

[22] H. Mark, Der feste Körper, 1938.

[23] C. S. Conrad, Diplomarbeit, Georg-August-Universität Göttingen, 2009.

[24] J. Branderup, E. H. Immergut, Polymer Handbook, John Wiley \& Sons, Inc., 1989.

[25] J. F. Quinn, R. P. Chaplin, T. P. Davis, J. Polym. Sci., Part A: Polym. Chem. 2002, 40(17), 2956-2966.

[26] T. R. Darling, T. P. Davis, M. Fryd, A. a. Gridnev, D. M. Haddleton, S. D. Ittel, R. R. 
Matheson, G. Moad, E. Rizzardo, J. Polym. Sci., Part A: Polym. Chem. 2000, 38(10), 1709 .

[27] M. Szwarc, Nature 1956, 178(4543), 1168-1169.

[28] M. Szwarc, J. Polym. Sci., Part A: Polym. Chem. 2000, 38(10), 1710.

[29] A. D. Jenkins, R. G. Jones, G. Moad, Pure Appl. Chem. 2010, 82(2), 483-491.

[30] G. Moad, E. Rizzardo, D. Solomon, Polym. Bull. 1982, 6(11-12), 589-593.

[31] D. H. Solomon, P. Cacioli, E. Rizzardo, EP0135280, 1985.

[32] L. Tebben, A. Studer, Angew. Chem. 2011, 123(22), 5138-5174.

[33] A. Studer, T. Schulte, Chem. Rec. 2005, 5(1), 27-35.

[34] J.-S. Wang, K. Matyjaszewski, J. Am. Chem. Soc. 1995, 117(20), 5614-5615.

[35] M. Kato, M. Kamigaito, M. Sawamoto, T. Higashimura, Macromolecules 1995, 28(5), 1721-1723.

[36] K. Matyjaszewski, J. Xia, Chem. Rev. 2001, 101(9), 2921-2990.

[37] J. Chiefari, Y. K. B. Chong, F. Ercole, J. Krstina, J. Jeffery, T. P. T. Le, R. T. A. Mayadunne, G. F. Meijs, C. L. Moad, G. Moad, E. Rizzardo, S. H. Thang, C. South, Macromolecules 1998, 31, 5559-5562.

[38] T. P. Le, G. Moad, E. Rizzardo, S. H. Thang, WO 98/01478, 1998.

[39] P. Corpart, D. Charmot, T. Biadatti, D. Michelet, WO 98/58974, 1998.

[40] G. Moad, J. Chiefari, B. Y. Chong, J. Krstina, R. T. Mayadunne, A. Postma, E. Rizzardo, S. H. Thang, Polym. Int. 2000, 49(9), 993-1001.

[41] G. Moad, E. Rizzardo, S. H. Thang, Aust. J. Chem. 2006, 59(10), 669.

[42] G. Moad, E. Rizzardo, S. H. Thang, Polymer 2008, 49(5), 1079-1131.

[43] G. Moad, E. Rizzardo, S. H. Thang, Aust. J. Chem. 2009, 62(11), 1402. 
[44] G. Moad, M. Benaglia, M. Chen, J. Chiefari, Y. K. Chong, D. J. Keddie, E. Rizzardo, S. H. Thang, ACS Symp. Ser. 2011, 1066, 81-102.

[45] G. Moad, E. Rizzardo, S. H. Thang, The Strem Chemiker 2011, 25(1), 2-10.

[46] J. F. Quinn, T. P. Davis, E. Rizzardo, Chem. Commun. 2001, (11), 1044-1045.

[47] H. Hussain, B. H. Tan, C. S. Gudipati, Y. Liu, C. B. He, T. P. Davis, J. Polym. Sci., Part A: Polym. Chem. 2008, 46(16), 5604-5615.

[48] C. D. Petruczok, R. F. Barlow, D. A. Shipp, J. Polym. Sci., Part A: Polym. Chem. 2008, 46(21), 7200-7206.

[49] M. Benaglia, J. Chiefari, Y. K. Chong, G. Moad, E. Rizzardo, S. H. Thang, J. Am. Chem. Soc. 2009, 131(20), 6914-6915.

[50] M. Benaglia, M. Chen, Y. K. Chong, G. Moad, E. Rizzardo, S. H. Thang, Macromolecules 2009, 42(24), 9384-9386.

[51] D. J. Keddie, C. Guerrero-Sanchez, G. Moad, E. Rizzardo, S. H. Thang, Macromolecules 2011, 44, 6738-6745.

[52] T. Arita, M. Buback, P. Vana, Macromolecules 2005, 38(19), 7935-7943.

[53] M. J. Monteiro, J. Polym. Sci., Part A: Polym. Chem. 2005, 43(15), 3189-3204.

[54] Akzo Nobel, Initiators for High Polymers, Techn. Ber., Akzo Nobel, 2006.

[55] Y. K. Chong, J. Krstina, T. P. T. Le, G. Moad, A. Postma, E. Rizzardo, S. H. Thang, Macromolecules 2003, 36(7), 2256-2272.

[56] M. Buback, F. Gu, G. T. Russell, P. Vana, Macromolecules 2009, 42(3), 652-662.

[57] Bibliographisches Institut, Duden, 2013.

[58] L. R. G. Treloar, The Physics of Rubber Elasticity, 3rd Aufl., (Hrsg.: C. E. H. Bawn, H. Fröhlich, P. B. Hirsch, N. F. Mott), Clarendon Press, Oxford, 1975, 1-310.

[59] (Hrsg.: A. S. Dunn), Rubber and Rubber Elasticity, (Hrsg.: A. S. Dunn), John Wiley \& Sons, Inc., New York, 1974, 1-211. 
[60] A. N. Gent, J. Polym. Sci.: Pol. Sym. 1974, 48, 1-17.

[61] Y. Gnanou, M. Fontanille, Organic and Physical Chemistry of Polymers, John Wiley \& Sons, Inc., Hoboken, New Jersey, 2008, 1-617.

[62] VDI Gesellschaft Entwicklung Kontrusktion Vertrieb, Konstruieren reyclinggerechter technischer Produkte, Grundlagen und Gestaltungsregeln, Techn. Ber., Verein Deutscher Ingenieure, 2000.

[63] M. Rätzsch, W. Schneider, D. Musche, J. Polym. Sci., Part A: Polym. Chem. 1971, 9, 785-790.

[64] L. J. Young, T. Dow, J. Polym. Sci., Part A: Polym. Chem. 1961, 54, 411-455.

[65] F. T. Wall, R. W. Powers, G. D. Sands, G. S. Stent, J. Am. Chem. Soc. 1948, 70(3), 1031-1037.

[66] F. E. Brown, G. E. Ham, J. Polym. Sci., Part A: Polym. Chem. 1964, 2(8), 3623-3632.

[67] A. W. Snow, E. E. Foos, Synthesis 2003, 2003(04), 0509-0512.

[68] G. D'Aprano, C. Henry, A. Godt, G. Wegner, Macromol. Chem. Phys. 1998, 199(12), 2777-2783.

[69] N. P. Neureiter, F. G. Bordwell, J. Am. Chem. Soc. 1960, 82(20), 5354-5358.

[70] J. H. Chapman, L. N. Owen, J. Chem. Soc. 1950, 579-585.

[71] Y. Sakai, E. Nishiwaki, K. Shishido, M. Shibuya, Tetrahedron Lett. 1991, 32(34), 4363-4366.

[72] R. Sato, T. Goto, Y. Takikawa, S. Takizawa, Synthesis 1980, 8, 615.

[73] A. Stoll, Dissertation, Ludwig-Maximilians-Universtität München, 2007.

[74] C. Barner-Kowollik, M. Buback, B. Charleux, M. L. Coote, M. Drache, T. Fukuda, A. Goto, B. Klumperman, A. B. Lowe, J. B. Mcleary, G. Moad, M. J. Monteiro, R. D. Sanderson, M. P. Tonge, P. Vana, P. Marie, Polymer 2006, 44, 5809-5831.

[75] B. Springer, Diplomarbeit, Georg-August-Universität Göttingen, 2007. 
[76] B. Springer, Dissertation, Georg-August-Universität Göttingen, 2011.

[77] J. Meijer, P. Vermeer, L. Brandsma, Recueil des Travaux Chimiques des Pays-Bas 1973, 92(4), 601-604.

[78] P. Beslin, A. Dlubala, G. Levesque, Synth. Commun. 1987, 9(9), 835-837.

[79] F. Becke, H. Hagen, US 3636089, 1972.

[80] R. W. Bost, W. J. Mattox, J. Am. Chem. Soc. 1930, 52(1), 332-335.

[81] S. H. Thang, Tetrahedron Lett. 1999, 40(12), 2435-2438.

[82] B. S. Pedersen, S. Scheibye, K. Clausen, S.-O. Lawesson, Bull. Soc. Chim. Belg. 1978, 87, 293-297.

[83] J. C. Godt, R. E. Wann, J. Org. Chem. 1961, 26(10), 4047-4051.

[84] A. Theis, A. Feldermann, N. Charton, M. H. Stenzel, T. P. Davis, C. BarnerKowollik, Macromolecules 2005, 38(7), 2595-2605.

[85] F. Duus, in Comprehensive Organic Chemistry, (Herausgegeben von D. Barton, W. D. Ollis), 1 Aufl., Pergamon, New York, 1979, 432.

[86] I. Degani, R. Fochi, A. Gatti, V. Regondi, Synthesis 1986, 11, 894-899.

[87] A. Sugawara, M. Shirahata, S. Sato, R. Sato, Bull. Chem. Soc. Jpn. 1984, 57(11), 3353-3354.

[88] E. Wertheim, J. Am. Chem. Soc. 1926, 48, 826-830.

[89] A. W. M. Lee, W. H. Chan, H. C. Wong, Synth. Commun. 1988, 18, 1531-1536.

[90] B. Tamami, A. R. Kiasat, J. Chem. Res., Synop. 1998, 454-455.

[91] S. Tamami, A. R. Kiasat, Iran. Polym. J. 1999, 8(1), 12-17.

[92] A. R. Kiasat, M. F. Mehrjardi, J. Chin. Chem. Soc. 2008, 55(3), 639-642.

[93] N. Aoyagi, B. Ochiai, H. Mori, T. Endo, Synlett 2006, 0636-0638. 
[94] N. Aoyagi, T. Endo, Polymer 2009, 47(15), 3702-3709.

[95] C. Boyer, M. R. Whittaker, K. Chuah, J. Liu, T. P. Davis, Langmuir 2010, 22(15), 2721-2730.

[96] C. H. Sohn, C. K. Chung, S. Yin, P. Ramachandran, J. a. Loo, J. L. Beauchamp, J. Am. Chem. Soc. 2009, 131(15), 5444-59.

[97] T.-C. Zheng, M. Burkart, D. E. Richardson, Tetrahedron Lett. 1999, 40, 603-606.

[98] E. Block, E. V. Dikarev, R. S. Glass, J. Jin, B. Li, X. Li, S.-Z. Zhang, J. Am. Chem. Soc. 2006, 128(46), 14949-14961.

[99] G. Gody, C. Rossner, J. Moraes, P. Vana, T. Maschmeyer, S. Perrier, J. Am. Chem. Soc. 2012, 134, 12596-12603.

[100] J. T. Lai, D. Filla, R. Shea, Macromolecules 2002, 35(18), 6754-6756.

[101] H. M. Etmimi, M. P. Tonge, R. D. Sanderson, J. Polym. Sci., Part A: Polym. Chem. 2011, 49, 1621-1632.

[102] R. J. Amir, S. Zhong, D. J. Pochan, C. J. Hawker, J. Am. Chem. Soc. 2009, 131(39), 13949-51.

[103] M. B. Andrus, J. Liu, Tetrahedron Lett. 2006, 47(32), 5811-5814.

[104] W. R. Nummy, US 2947731, 1960.

[105] C. M. Starks, J. Am. Chem. Soc. 1971, 93(1), 195-199.

[106] C. M. Starks, ACS Symp. Ser. 1987, 326, 1-7.

[107] J. M. McIntosh, J. Chem. Educ. 1978, 55(4), 235-238.

[108] W. P. Reeves, M. R. White, D. Bier, J. Chem. Educ. 1978, 55(1), 56.

[109] W. P. Weber, L. Angeles, G. W. Gokel, J. Chem. Educ. 1978, 55(7), 429-433.

[110] J. W. Hill, J. Corredor, J. Chem. Educ. 1980, 57(11), 822. 
[111] M. E. Childs, W. P. Weber, J. Org. Chem. 1976, 41(21), 3486-3487.

[112] M. J. Joralemon, R. K. O’Reilly, J. B. Matson, A. K. Nugent, C. J. Hawker, K. L. Wooley, Macromolecules 2005, 38(13), 5436-5443.

[113] G. W. Gokel, W. P. Weber, J. Chem. Educ. 1971, 55(6), 350-354.

[114] M. Rubinstein, R. H. Colby, Polymer Physica, Oxford University Press Inc., New York, 2003, 263.

[115] T. Inasaki, K. Nomura, JP 2006199827, 2006.

[116] K. Nomura, JP 2006048993, 2006.

[117] S. Ichikawa, JP 2000356702, 2000.

[118] G. Wulff, I. Schulze, Israel J. Chem. 1978, 17, 291-297.

[119] A. Ray, S. M. Boyle, US 2011/032804, 2011.

[120] D. A. Lewis, P. A. Small, GB 843611, 1960.

[121] A. J. Heidenreich, J. E. Puskas, J. Polym. Sci., Part A: Polym. Chem. 2008, 46, 76217627.

[122] Z. Wang, J. He, Y. Tao, L. Yang, H. Jiang, Y. Yang, Macromolecules 2003, 36, 74467452.

[123] K. S. Khuong, W. H. Jones, W. a. Pryor, K. N. Houk, J. Am. Chem. Soc. 2005, 127(4), 1265-77.

[124] J. Chiefari, R. T. a. Mayadunne, C. L. Moad, G. Moad, E. Rizzardo, A. Postma, S. H. Thang, Macromolecules 2003, 36(7), 2273-2283.

[125] C. Zhang, Y. Zhou, Q. Liu, S. Li, Macromolecules 2011, 44, 2034-2049.

[126] M. R. Whittaker, Y.-K. Goh, H. Gemici, T. M. Legge, S. Perrier, M. J. Monteiro, Macromolecules 2006, 39(26), 9028-9034. 
[127] E. Rizzardo, J. Chiefari, M. Benaglia, S. H. Thang, G. Moad, WO 2010/083569, 2010.

[128] H. E. Gottlieb, V. Kotlyar, A. Nudelman, J. Org. Chem. 1997, 62(21), 7512-7515.

[129] G. R. Fulmer, A. J. M. Miller, N. H. Sherden, H. E. Gottlieb, A. Nudelman, B. M. Stoltz, J. E. Bercaw, K. I. Goldberg, Organometallics 2010, 29(9), 2176-2179.

[130] M. Buback, A. Feldermann, C. Barner-Kowollik, I. Lacik, Macromolecules 2001, 34, 5439-5448.

[131] D. P. Gamblin, P. Garnier, S. J. Ward, N. J. Oldham, A. J. Fairbanks, B. G. Davis, Org. Biomol. Chem. 2003, 1(21), 3642-4.

[132] K. Hagiya, M. Hirano, WO 2011/040131, 2011. 



\section{Danksagung}

Ich möchte mich bei meinem Doktorvater, Prof. Dr. Philipp Vana, für die interessante Themenstellung und die hilfreichen Diskussionen und Anregungen bedanken.

Prof. Dr. Michael Buback danke ich für die Übernahme des Korreferats und die Diskussionsbereitschaft.

Der Continental Reifen GmbH, insbesondere Frau Dr. Lena Müller, Frau Dr. Katharina Herzog, Frau Dr. Sabine Müller, Frau Dr. Carla Recker und Frau Dr. Julia Große, danke ich für die finanzielle Unterstützung dieses Projektes und die Diskussionsbereitschaft während der Meilensteintreffen.

Meinem Bachelor-Studenten Georgios Dodekatos, der sich trotz vieler Rückschläge nicht hat entmutigen lassen, danke ich für die Mitarbeit an diesem Projekt, genauso wie allen Abteilungspraktikanten für ihre motivierte Mitarbeit im Labor.

Die Analysen, die im Rahmen dieser Doktorarbeit durchgeführt wurden, entstanden in den analytischen Abteilungen der chemischen Institute. Stellvertretend für die zahlreichen fleißigen Mitarbeiter möchte ich mich bei Dr. Michael John für die Betreuung des NMR-Autosamplers, Reinhard Machinek für die Messung der Polymer-NMRSpektren, Dr. Holm Frauendorf für die massenspektrometrische Analytik und Angelika Wraage für die Elementaranalysen bedanken. Den Mitarbeitern der glastechnischen, mechanischen und elektronischen Werkstätten sei für die schnelle und unkomplizierte Erledigung von Reparaturen und Maßanfertigungen ebenfalls gedankt.

Dennis Hübner, Björn Malte Sölter, Hendrik Kattner, Dr. Nadja Förster und Dr. Galina Pestel haben diese Arbeit ganz oder in Ausschnitten korrigiert und teilweise dafür ihre Freizeit geopfert, wofür ich mich bei euch herzlich bedanken möchte! 
Bastian Ebeling stand mir bei $\mathrm{LT}_{\mathrm{E}} \mathrm{X}$-Fragen mit Rat und Tat zur Seite und Hendrik Kattner bei Kinetik-Fragen - danke dafür!

Das Labor im 4. Stock habe ich mit zahlreichen Kollegen geteilt, von Dr. Martin Mänz über meinen Buddy Dennis Hübner bis zu Jan Schwellenbach. Ihnen allen, meinen Bürokollegen im 4. und im 3. Stock sowie den restlichen Mitgliedern der Arbeitsgruppen TuMChem und MMC, besonders aber Dr. Björn Springer und Dr. Nadja Förster, danke ich für die unterhaltsame Zeit. Darüber hinaus danke ich Percy Spencer, dem Erfinder der Mikrowelle, der mir eine lohnende Alternative zur Nordmensa verschafft hat und ohne den unsere Mittagsrunde aufgeschmissen gewesen wäre. Danke, Mädels, für viele leckere Gerichte und interessante Experimente!

Viele gute Freunde haben mich während der Promotion begleitet; beim Doppelkopf, auf Spieleabenden und Stricktreffen, in die Sauna und zum Sport. Ihnen allen, besonders Karsten Behrendt, Dr. Nadja Förster, Dr. Galina Pestel und Katharina Tietz, danke ich für die schöne gemeinsame Freizeit und den Spaß neben der Arbeit.

Bei meinen Eltern möchte ich mich für die Unterstützung und den Rückhalt während 22 Jahren Ausbildung, von der Einschulung bis zum Abschluss der Promotion, bedanken.

Schlussendlich danke ich Kai, der weiß wie sehr erfolglose Experimente frusten und der es immer wieder geschafft hat, mich zum Weitermachen zu motivieren. Danke für deine Unterstützung! 


\section{Curriculum Vitae - Cathrin Sonja Conrad}

Geboren

16.12.1984 in Hannover

Staatsangehörigkeit

Deutsch

\section{Akademischer Werdegang}

29.10.2013

Disputation

$10 / 2009-09 / 2013$

Promotion mit dem Thema: „Polybutadien und Butadien enthaltende Copolymere mit gezielt eingebauten vulkanisierbaren Gruppen durch RAFT-Polymerisation“ im Arbeitskreis von Prof. Dr. Philipp Vana, Institut für Physikalische Chemie der Georg-August-Universität Göttingen.

$08 / 2009$

Diplomprüfung, Note: „sehr gut“.

$11 / 2008-05 / 2009$

Diplomarbeit mit dem Thema „Polybutadien und Butadien enthaltende Copolymere mit gezielt eingebauten vulkanisierbaren Gruppen durch RAFT-Polymerisation“ im Arbeitskreis von Prof. Dr. Philipp Vana, Institut für Physikalische Chemie der Georg-August-Universität Göttingen.

04/2008 - 07/2008 Auslandsaufenthalt an der Universität Stockholm, Institut für Organische Chemie, Abteilung Prof. Dr. Kálmán Szabó.

$10 / 2006$

Diplomvorprüfung, Note: „sehr gut“.

$10 / 2004$

Studienbeginn im Diplomstudiengang Chemie an der GeorgAugust-Universität Göttingen.

$06 / 2004$

Abitur am Gymnasium Burgdorf, Note: 1,6.

\section{Veröffentlichungen}

2009

J. Aydin, C. S. Conrad, K. J. Szabó, „Stereoselective Pincer-Complex Catalyzed C-H Functionalization of Benzyl Nitriles under Mild Conditions. An Efficient Route to $\beta$-Aminonitriles." Org. Lett. 2008, 22, 5175-5178. 
